UNIVERSIDAD POLITÉCNICA DE MADRID

ESCUELA TÉCNICA SUPERIOR DE INGENIEROS DE

TELECOMUNICACIÓN

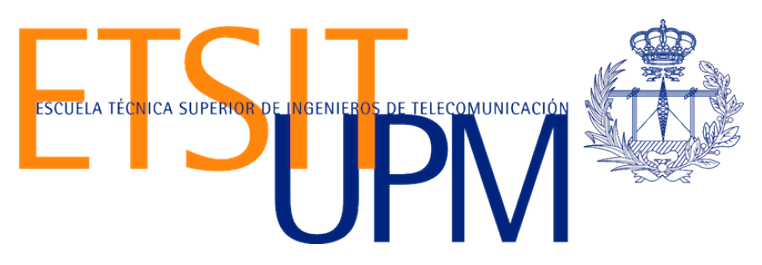

AUTOMATED WORDLENGTH OPTIMIZATION

FRAMEWORK FOR MULTI-SOURCE STATISTICAL

INTERVAL-BASED ANALYSIS OF NONLINEAR SYSTEMS

WITH CONTROL-FLOW STRUCTURES

Ph.D. THESIS

Enrique Sedano Algarabel

Ingeniero en Informática

Máster en Investigación en Informática

2016 


\author{
DEPARTAMENTO DE INGENIERÍA ELECTRÓNICA \\ ESCUELA TÉCNICA SUPERIOR DE INGENIEROS DE \\ TELECOMUNICACIÓN \\ UNIVERSIDAD POLITÉCNICA DE MADRID
}

\title{
AUTOMATED WORDLENGTH OPTIMIZATION \\ FRAMEWORK FOR MULTI-SOURCE STATISTICAL INTERVAL-BASED ANALYSIS OF NONLINEAR SYSTEMS WITH CONTROL-FLOW STRUCTURES
}

\section{Ph.D. THESIS}

\author{
Author: \\ Enrique Sedano Algarabel \\ Ingeniero en Informática \\ Máster en Investigación en Informática
}

\author{
Advisors: \\ Juan Antonio López Martín \\ Profesor Contratado Doctor del Dpto. de Ingeniería Electrónica \\ Universidad Politécnica de Madrid \\ Carlos Carreras Vaquer \\ Profesor Titular del Dpto. de Ingeniería Electrónica \\ Universidad Politécnica de Madrid
}


PH.D. THESIS: Automated word-length optimization framework for multi-source statistical interval-based analysis of non-linear systems with control-flow structures

AUTHOR: Enrique Sedano Algarabel

ADVISORS: Juan Antonio López Martín

Carlos Carreras Vaquer

El tribunal nombrado para juzgar la Tesis arriba indicada, compuesto por los siguientes doctores:

PRESIDENTE:

VOCALES:

SECRETARIO:

acuerdan otorgarle la calificación de:

Madrid, a 7 de marzo de 2016

El Secretario del Tribunal 
To my family and friends, especially to my three girls. 
They were magi because they had a tremendous knowledge, so much indeed that quantity had finally been transmuted into quality, and they had come into a different relationship with the world than ordinary people. They worked in an Institute that was dedicated above all to the problems of human happiness and the meaning of human life, and even among them, not one knew exactly what was happiness and what precisely was the meaning of life. So they took it as a working hypothesis that happiness lay in gaining perpetually new insights into the unknown and the meaning of life was to be found in the same process.

Boris and Arkady Strugatsky, Monday Begins on Saturday 


\section{Table of Contents}

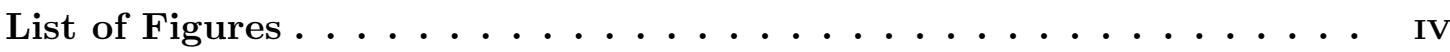

List of Tables $\ldots \ldots \ldots \ldots \ldots \ldots \ldots \ldots$ VI

List of Acronyms ...................... VII

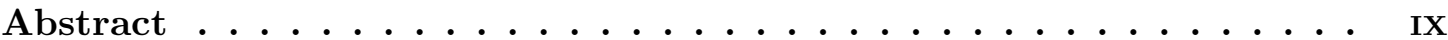

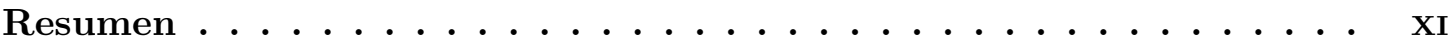

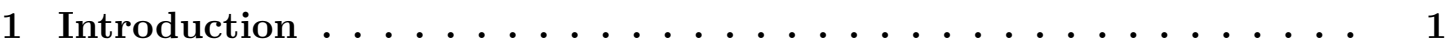

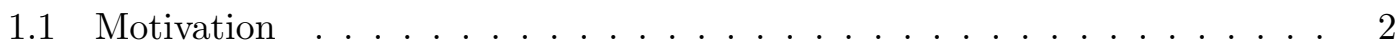

1.2 Thesis contributions . . . . . . . . . . . . . . . 4

1.3 Book structure . . . . . . . . . . . . . . . . . . 6

2 Dynamic range and round-off noise estimation $\ldots \ldots \ldots \ldots$

2.1 Dynamic range and fractional precision modelling . . . . . . . . . . . . . 9

2.1 .1 Dynamic range estimation . . . . . . . . . . . . . . . . 10

2.1.2 Fractional precision analysis . . . . . . . . . . . . . . 11

2.2 Word-length optimization . . . . . . . . . . . . . . . . . . . 14

2.2.1 Search-based optimization techniques . . . . . . . . . . . . 14

2.2.2 Alternative optimization approaches . . . . . . . . . . . . 17

2.2 .3 Quantization noise metrics . . . . . . . . . . . . . . . . 19

2.2 .4 Optimization objectives . . . . . . . . . . . . . . 20

2.2 .5 Mixed approaches . . . . . . . . . . . . . . . . . 21

2.3 Computational scalability . . . . . . . . . . . . . . . . 22

2.4 Fixed-point tools and frameworks . . . . . . . . . . . . . . . . . 23

2.5 Conclusions . . . . . . . . . . . . . . . . . . 26

3 Quantization of systems with control flow structures . . . . . . 28

3.1 Theoretical background . . . . . . . . . . . . . . . . . . . . . 29

3.1 .1 Affine arithmetic . . . . . . . . . . . . . . 30

3.1 .2 Modified affine arithmetic . . . . . . . . . . . . . . . 30

3.1.3 Polynomial chaos expansion . . . . . . . . . . . . . . . . . 31

3.1.4 Multi-element generalized polynomial chaos . . . . . . . . . . . . 34 
3.2 Dynamic range estimation in systems with control-flow structures . . . . . 36

3.2 .1 Control-flow structures . . . . . . . . . . . . . . 37

3.2 .2 Domain partitioning . . . . . . . . . . . . . . 40

3.2 .3 Propagation of the affine forms . . . . . . . . . . . . 42

3.2.4 Computation of the system statistics . . . . . . . . . . . . 43

3.2.5 Example: Absolute value of a multiplication . . . . . . . . . . . . 44

3.3 Round-off noise modelling in systems with control-flow structures . . . . . 46

3.3.1 Modelling of the quantization noise sources . . . . . . . . . 47

3.3.2 RON coefficients propagation example . . . . . . . . . . . . . 48

3.3 .3 Computation of the RON statistics . . . . . . . . . . . 50

3.3.4 Example: Quantization of absolute value of a multiplication . . . . 51

3.4 Clustered noise injection . . . . . . . . . . . . . . . . . . . 53

3.4 Methodology ..................... 54

3.4.2 Grouping of noise sources and impact on accuracy . . . . . . . 55

3.4.3 Hierarchical partitioning . . . . . . . . . . . . . . . . . . 57

3.5 Conclusions . . . . . . . . . . . . . . . . . . . . . 61

4 System modelling case studies . . . . . . . . . . . . 62

4.1 Initial study: Interferometer . . . . . . . . . . . . . . . . . . 63

4.2 Choice operators: Teager $+\ldots \ldots \ldots$. . . . . . . . . . . 64

4.2 .1 Teager+: Dynamic range estimation . . . . . . . . . . . . 64

4.2 .2 Teager+: RON modelling . . . . . . . . . . . . . . . . . . . . . . . 66

4.3 Loop structures: Accumulator . . . . . . . . . . . . . . . . . 70

4.3.1 Accumulator: Dynamic range estimation . . . . . . . . . . . . 70

4.3 .2 Accumulator: RON modelling . . . . . . . . . . . . . . . . . . . . . . . . . . . . 73

4.4 Nested control-flow structures: Selector . . . . . . . . . . . . . . . . . . . 74

4.4.1 Selector: Dynamic range estimation . . . . . . . . . . . 74

4.4 .2 Selector: RON modelling . . . . . . . . . . . . . . . 76

4.5 Clustered noise injection: L-Opchain . . . . . . . . . . . . . . . 77

4.6 Putting it all together: Compounded . . . . . . . . . . . . . . . . . 80

4.6.1 Compounded: Dynamic range estimation . . . . . . . . . . . 81

4.6 .2 Compounded: RON modelling . . . . . . . . . . . . . 82

4.7 Conclusions . . . . . . . . . . . . . . . . . 82

5 Word-length optimization . . . . . . . . . . . . . 84

5.1 Definitions . . . . . . . . . . . . . . . . . 85

5.2 Interpolative method . . . . . . . . . . . . . 86

5.2 .1 Methodology . . . . . . . . . . . . 86

5.2 .2 Minimization of the number of simulations . . . . . . . . . 89

5.2 .3 Interpolative: Case study . . . . . . . . . . . . . . . . 90

5.3 Incremental method . . . . . . . . . . . . . . . . . . . . . . 91 
5.3.1 Confidence intervals and number of simulations . . . . . . . . . . 92

5.3 .2 Methodology . . . . . . . . . . . . . . . . . . 93

5.3 .3 Incremental: Case study . . . . . . . . . . . . . . . . . . . . 95

5.4 Combined approach . . . . . . . . . . . . . . . . . . 96

5.5 Experimental results . . . . . . . . . . . . . . . . . . . 97

5.6 Conclusions . . . . . . . . . . . . . . . . . . . . 99

6 The HOPLITE framework $\ldots \ldots \ldots \ldots$

6.1 Work flow . . . . . . . . . . . . . . . . . . . . . . . 101

6.2 Implementation . . . . . . . . . . . . . . . . . . . . . . . . . . . 101

6.2.1 Programming language choice . . . . . . . . . . . . . . . . . 103

6.2 .2 Configuration files . . . . . . . . . . . . . . . . . . . . . 104

6.2.3 System graph representation . . . . . . . . . . . . . . . . 105

6.2.4 Modules and interfaces . . . . . . . . . . . . . . . . . . . 106

6.2 .5 Calling sequence . . . . . . . . . . . . . . . . . . . 110

6.3 Case study . . . . . . . . . . . . . . . . . . . . . . . 111

6.4 Extending HOPLITE . . . . . . . . . . . . . . . . . . . . . . . . . . 114

6.4.1 The Phalanx case . . . . . . . . . . . . . . . . . . . . . . 114

6.5 Conclusions . . . . . . . . . . . . . . . . . . 116

7 Conclusions and future work $\ldots \ldots \ldots \ldots \ldots$

7.1 Future work . . . . . . . . . . . . . . . . . . . . . . 119

Appendix A Computation of the C matrix ........... 122

Appendix B Execution times in HOPLITE . . . . . . . . . 126

Bibliography . . . . . . . . . . . . . . . . . . 129 


\section{List of Figures}

1.1 Fixed-point word-length optimization flow. . . . . . . . . . . . 3

3.1 Nodes representation of a control-flow structure. . . . . . . . . . . . 38

3.2 Derivative execution paths from a choice operator. . . . . . . . . . . 39

3.3 Derivative execution paths from a loop structure. . . . . . . . . . . . . . 39

3.4 Example of a decomposed domain. . . . . . . . . . . . . . . . . . . 41

3.5 Domain decomposition for $x>y$ with different values of $J_{l i m} \ldots \ldots$. . . 41

3.6 Dynamic range of an intermediate variable across an unrolled loop. . . . . 44

3.7 Absolute value operation and execution paths corresponding to sub-domains. 45

3.8 Methodology for RON computation in systems with control-flow structures. 47

3.9 Two different ways of introducing AWN sources to an operation. . . . . . 48

3.10 Fractional word-length to derivative DFGs nodes assignment examples. . . 49

3.11 Absolute value example paths with noise sources. . . . . . . . . . . 51

3.12 Number of terms in a PCE base function of the order and number of RVs. 54

3.13 Iterative noise injection algorithm flow. . . . . . . . . . . . . . 55

3.14 Simple system without and with noise sources. . . . . . . . . . . . 56

3.15 Example of the hierarchical decomposition of a DFG. . . . . . . . . . . . . 59

4.1 Phase measuring stage of the TJ-II interpolation algorithm with quantization inputs. . . . . . . . . . . . . . . . . 63

4.2 Basic Blocks diagram and derivative DFGs of the Teager case study. . . . 64

4.3 Partitions per decision output direction in the Teager case study. . . . . . 65

4.4 Derivarive DFGs from Figure 4.2 including the noise sources. . . . . . . . 68

4.5 Basic Blocks diagram of the loop case study. . . . . . . . . . . . . . 71

4.6 Partitions per iteration of the loop case study. . . . . . . . . . . . . . . 72

4.7 Data Flow Graphs generated by the analysis of the loop case study. . . . 72

4.8 Partitions per condition and iteration of the nested conditionals case study. 75

4.9 Data Flow Graphs generated by the analysis of the nested conditionals case study. . . . . . . . . . . . . . . . . . 76

4.10 L-Opchain case study DFG. . . . . . . . . . . . . . . . . . . 78

4.11 Basic Blocks diagram for the compounded case study. . . . . . . . . . . 81

5.1 Interpolative method algorithm flow. . . . . . . . . . . . . . . . 87

5.2 Evolution of normalized error for different values of $b . \ldots \ldots 8$ 
5.3 Block diagram of a second order IIR filter. . . . . . . . . . . . . . . . . 90

5.4 Incremental method algorithm flow. . . . . . . . . . . . . . . . . . . 94

5.5 Normalized speedups with respect to the preplanned search. . . . . . . . . 98

6.1 HOPLITE framework work flow. . . . . . . . . . . . . . . . . . . . 102

6.2 HOPLITE call sequence. . . . . . . . . . . . . . . . . . . . . 110

6.3 Basic Blocks diagram of the case study. . . . . . . . . . . . . . . . . . 112

6.4 Resulting execution paths from case study. . . . . . . . . . . . . . 113

6.5 Phalanx/HOPLITE integration flow. . . . . . . . . . . . . . . . . 115 


\section{List of Tables}

2.1 Techniques for the evaluation of the Dynamic Range (DR) and RON. . . 12

2.2 Classification of optimization techniques. . . . . . . . . . . . . . 18

3.1 Coefficient propagation rules of Affine Arithmetic. . . . . . . . . . . . . 30

3.2 Types of RVs and their associated sets of orthonormal polynomials. . . . 32

3.3 Coefficient propagation rules of PCE basic operations. . . . . . . . . . . . 43

3.4 PCE polynomials for sub-domain inputs in absolute value. . . . . . . . . . 45

3.5 Propagation of signal and RON terms through a MAC operation. . . . . . 50

4.1 SQNR obtained for different fractional word-lengths after quantizing the whole interferometer case study. . . . . . . . . . . . . . . . 63

4.2 PCE coefficients for Teager + with $J_{l i m}=10^{-4} \ldots \ldots \ldots \ldots$. . . . . 67

4.3 System statistics for different values of $J_{l i m}$ in the Teager+ case study. . . 67

4.4 System statistics for different values of $J_{\text {lim }}$ in the Accumulator case study. 73

$4.5 f_{w q}$ assignment to each noise source in the Accumulator case study. . . . . 74

4.6 System statistics for different values of $J_{l i m}$ in the Selector case study. . . 76

4.7 Results for various cluster sizes. . . . . . . . . . . . . . . . . 80

4.8 Estimated system statistics for different values of $J_{\text {lim }}$ in the Compounded case study. . . . . . . . . . . . . . . . . . . . . . . 82

$5.1 M_{d}$ matrix for a second-order IIR filter. . . . . . . . . . . . . . . 91

5.2 Evolution of the interpolative method for a second-order IIR filter. . . . . 91

5.3 Distribution of the execution times among the optimization stages. . . . . 96

5.4 Execution times for different benchmarks. . . . . . . . . . . . . . . 98

6.1 Language requisites and availability. . . . . . . . . . . . . . . . 103

A.1 Comparison of methods for C matrix computation. . . . . . . . . . . 125

B.1 Steps and execution time of HOPLITE for the CoU_function case study. . 127 


\section{List of Acronyms}

$\begin{array}{ll}\text { AA } & \text { Affine Arithmetic } \\ \text { AD } & \text { Automatic Differentiation } \\ \text { AS } & \text { Architectural Synthesis } \\ \text { ASIC } & \text { Application Specific Integrated Circuit } \\ \text { AT } & \text { Arithmetic Transforms } \\ \text { AWN } & \text { Additive White Noise } \\ \text { BB } & \text { Basic Block } \\ \text { BER } & \text { Bit Error Rate } \\ \text { DFG } & \text { Data Flow Graph } \\ \text { DNM } & \text { Discrete Noise Model } \\ \text { DSP } & \text { Digital Signal Processing } \\ \text { EDA } & \text { Electronic Design Automation } \\ \text { FCC } & \text { Fixed-C Compiler } \\ \text { FM } & \text { Fiduccia and Mattheyses } \\ \text { FPGA } & \text { Field Programmable Gate Array } \\ \text { FWL } & \text { Fractional Word-Length } \\ \text { GA } & \text { Genetic Algorithms } \\ \text { GIA } & \text { General Interval Arithmetic } \\ \text { GMP } & \text { GNU Multi-Precision arithmetic library } \\ \text { GRASP } & \text { Greedy Randomized Adaptive Search Procedure } \\ \text { GPU } & \text { Graphics Processing Unit } \\ \text { HLS } & \text { Interval Arithmetic } \\ \text { IA } & \text { Karhunen-Lòeve Expansion } \\ \text { KLE } & \text { LTI }\end{array}$




$\begin{array}{ll}\text { MAA } & \text { Modified Affine Arithmetic } \\ \text { MCS } & \text { Monte-Carlo Sampling } \\ \text { ME-gPC } & \text { Multi-Element Generalized Polynomial Chaos } \\ \text { MILP } & \text { Mixed Integer Linear Programming } \\ \text { MM } & \text { Maximum Mismatch } \\ \text { MSE } & \text { Mean Square Error } \\ \text { mUWL } & \text { Minimal Uniform Word-Length vector } \\ \text { mWS } & \text { Minimum Word-length Solution } \\ \text { MWL } & \text { Multiple Word-Length optimization } \\ \text { PCE } & \text { Polynomial Chaos Expansion } \\ \text { PDF } & \text { Probability Density Function } \\ \text { PSD } & \text { Power Spectral Density } \\ \text { PT } & \text { Perturbation Theory } \\ \text { RON } & \text { Round-Off Noise } \\ \text { RV } & \text { Random Variable } \\ \text { SA } & \text { Simulated Annealing } \\ \text { SG } & \text { System Graph } \\ \text { SNA } & \text { Symbolic Noise Analysis } \\ \text { SNSM } & \text { Single Noise Source Model } \\ \text { SSD } & \text { Single Source Directed } \\ \text { SQNR } & \text { Wignal to Quantization Noise Ratio } \\ \text { UWL } & \text { Word-Length Optimization } \\ \text { WLO } & \text { WLV }\end{array}$




\section{Abstract}

Using fixed-point arithmetic is one of the most common design choices for systems where area, power or throughput are heavily constrained. In order to produce implementations where the cost is minimized without negatively impacting the accuracy of the results, a careful assignment of word-lengths is required. The problem of finding the optimal combination of fixed-point word-lengths for a given system is a combinatorial NP-hard problem to which developers devote between 25 and $50 \%$ of the design-cycle time. Reconfigurable hardware platforms such as FPGAs also benefit of the advantages of fixed-point arithmetic, as it compensates for the slower clock frequencies and less efficient area utilization of the hardware platform with respect to ASICs. As FPGAs become commonly used for scientific computation, designs constantly grow larger and more complex, up to the point where they cannot be handled efficiently by current signal and quantization noise modelling and word-length optimization methodologies.

In this Ph.D. Thesis we explore different aspects of the quantization problem and we present new methodologies for each of them:

The techniques based on extensions of intervals have allowed to obtain accurate models of the signal and quantization noise propagation in systems with non-linear operations. We take this approach a step further by introducing elements of MultiElement Generalized Polynomial Chaos (ME-gPC) and combining them with an stateof-the-art Statistical Modified Affine Arithmetic (MAA) based methodology in order to model systems that contain control-flow structures. Our methodology produces the different execution paths automatically, determines the regions of the input domain that will exercise them, and extracts the system statistical moments from the partial results. We use this technique to estimate both the dynamic range and the round-off noise in systems with the aforementioned control-flow structures. We show the good accuracy of our approach, which in some case studies with non-linear operators shows a $0.04 \%$ deviation respect to the simulation-based reference values.

A known drawback of the techniques based on extensions of intervals is the combinatorial explosion of terms as the size of the targeted systems grows, which leads to scalability problems. To address this issue we present a clustered noise injection technique that groups the signals in the system, introduces the noise terms in each group independently and then combines the results at the end. In this way, the number of noise sources in the system at a given time is controlled and, because of this, the combinato- 
rial explosion is minimized. We also present a multi-way partitioning algorithm aimed at minimizing the deviation of the results due to the loss of correlation between noise terms, in order to keep the results as accurate as possible.

This Ph.D. Thesis also covers the development of methodologies for word-length optimization based on Monte-Carlo simulations in reasonable times. We do so by presenting two novel techniques that explore the reduction of the execution times approaching the problem in two different ways: First, the interpolative method applies a simple but precise interpolator to estimate the sensitivity of each signal, which is later used to guide the optimization effort. Second, the incremental method revolves on the fact that, although we strictly need to guarantee a certain confidence level in the simulations for the final results of the optimization process, we can do it with more relaxed levels, which in turn implies using a considerably smaller amount of samples, in the initial stages of the process, when we are still far from the optimized solution. Through these two approaches we demonstrate that the execution time of classical greedy techniques can be accelerated by factors of up to $\times 240$ for small/medium sized problems.

Finally, this book introduces HOPLITE, an automated, flexible and modular framework for quantization that includes the implementation of the previous techniques and is provided for public access. The aim is to offer a common ground for developers and researches for prototyping and verifying new techniques for system modelling and word-length optimization easily. We describe its work flow, justifying the taken design decisions, explain its public API and we do a step-by-step demonstration of its execution. We also show, through an example, the way new extensions to the flow should be connected to the existing interfaces in order to expand and improve the capabilities of HOPLITE. 


\section{Resumen}

El uso de aritmética de punto fijo es una opción de diseño muy extendida en sistemas con fuertes restricciones de área, consumo o rendimiento. Para producir implementaciones donde los costes se minimicen sin impactar negativamente en la precisión de los resultados debemos llevar a cabo una asignación cuidadosa de anchuras de palabra. Encontrar la combinación óptima de anchuras de palabra en coma fija para un sistema dado es un problema combinatorio $N P$-hard al que los diseñadores dedican entre el $25 \mathrm{y}$ el $50 \%$ del ciclo de diseño. Las plataformas hardware reconfigurables, como son las FPGAs, también se benefician de las ventajas que ofrece la aritmética de coma fija, ya que éstas compensan las frecuencias de reloj más bajas y el uso más ineficiente del hardware que hacen estas plataformas respecto a los ASICs. A medida que las FPGAs se popularizan para su uso en computación científica los diseños aumentan de tamaño y complejidad hasta llegar al punto en que no pueden ser manejados eficientemente por las técnicas actuales de modelado de señal y ruido de cuantificación y de optimización de anchura de palabra.

En esta Tesis Doctoral exploramos distintos aspectos del problema de la cuantificación y presentamos nuevas metodologías para cada uno de ellos:

Las técnicas basadas en extensiones de intervalos han permitido obtener modelos de propagación de señal y ruido de cuantificación muy precisos en sistemas con operaciones no lineales. Nosotros llevamos esta aproximación un paso más allá introduciendo elementos de Multi-Element Generalized Polynomial Chaos (ME-gPC) y combinándolos con una técnica moderna basada en Modified Affine Arithmetic (MAA) estadístico para así modelar sistemas que contienen estructuras de control de flujo. Nuestra metodología genera los distintos caminos de ejecución automáticamente, determina las regiones del dominio de entrada que ejercitarán cada uno de ellos y extrae los momentos estadísticos del sistema a partir de dichas soluciones parciales. Utilizamos esta técnica para estimar tanto el rango dinámico como el ruido de redondeo en sistemas con las ya mencionadas estructuras de control de flujo y mostramos la precisión de nuestra aproximación, que en determinados casos de uso con operadores no lineales llega a tener tan solo una desviación del $0.04 \%$ con respecto a los valores de referencia obtenidos mediante simulación.

Un inconveniente conocido de las técnicas basadas en extensiones de intervalos es la explosión combinacional de términos a medida que el tamaño de los sistemas a estudiar crece, lo cual conlleva problemas de escalabilidad. Para afrontar este problema presen- 
tamos una técnica de inyección de ruidos agrupados que hace grupos con las señales del sistema, introduce las fuentes de ruido para cada uno de los grupos por separado y finalmente combina los resultados de cada uno de ellos. De esta forma, el número de fuentes de ruido queda controlado en cada momento y, debido a ello, la explosión combinatoria se minimiza. También presentamos un algoritmo de particionado multi-vía destinado a minimizar la desviación de los resultados a causa de la pérdida de correlación entre términos de ruido con el objetivo de mantener los resultados tan precisos como sea posible.

La presente Tesis Doctoral también aborda el desarrollo de metodologías de optimización de anchura de palabra basadas en simulaciones de Monte-Carlo que se ejecuten en tiempos razonables. Para ello presentamos dos nuevas técnicas que exploran la reducción del tiempo de ejecución desde distintos ángulos: En primer lugar, el método interpolativo aplica un interpolador sencillo pero preciso para estimar la sensibilidad de cada señal, y que es usado después durante la etapa de optimización. En segundo lugar, el método incremental gira en torno al hecho de que, aunque es estrictamente necesario mantener un intervalo de confianza dado para los resultados finales de nuestra búsqueda, podemos emplear niveles de confianza más relajados, lo cual deriva en un menor número de pruebas por simulación, en las etapas iniciales de la búsqueda, cuando todavía estamos lejos de las soluciones optimizadas. Mediante estas dos aproximaciones demostramos que podemos acelerar el tiempo de ejecución de los algoritmos clásicos de búsqueda voraz en factores de hasta $\times 240$ para problemas de tamaño pequeño/mediano.

Finalmente, este libro presenta HOPLITE, una infraestructura de cuantificación automatizada, flexible y modular que incluye la implementación de las técnicas anteriores y se proporciona de forma pública. Su objetivo es ofrecer a desarrolladores e investigadores un entorno común para prototipar y verificar nuevas metodologías de cuantificación de forma sencilla. Describimos el flujo de trabajo, justificamos las decisiones de diseño tomadas, explicamos su API pública y hacemos una demostración paso a paso de su funcionamiento. Además mostramos, a través de un ejemplo sencillo, la forma en que conectar nuevas extensiones a la herramienta con las interfaces ya existentes para poder así expandir y mejorar las capacidades de HOPLITE. 


\section{Chapter 1}

\section{Introduction}

The world was full of powerful men but as long as they played the same roles, they were as interchangeable as second-rate players speaking the same lines in the same theatre on different nights.

Neal Stephenson, Quicksilver

In an industry where time-to-market is critical, begin able to design and implement efficient and reliable Digital Signal Processing (DSP) systems can make the difference between success and failure. And still, finding a fast and general way for transforming floating-point system descriptions to efficient fixed-point implementations remains an open issue. Fixed-point arithmetic is widely used in DSP systems, specially when implemented on FPGAs and ASICs, due to its lower implementation cost and power consumption, and its higher performance than its floating-point alternative. But fixed-point arithmetic is limited in dynamic range and fractional precision, and we have to carry out exhaustive studies to determine appropriate fixed-point word-lengths and avoid overand underflow errors due to the round-off effects. The analysis and selection of optimized word-lengths is an important and time-consuming step in the design flow of DSP and VLSI systems. Studies indicate that fixed-point refinement can represent from $25 \%$ to $50 \%$ of the overall development time [CMJL05]. Thus, automating and accelerating that process is very desirable.

As the demand for performance and reliability increases and the target applications continue to grow in size, the Electronic Design Automation (EDA) methodologies need to evolve in order to fulfil the demands and expectations put in them. Researchers and developers need to find more inclusive ways for estimating the error due to fixedpoint arithmetic and faster techniques for optimizing the word-length allocation. Also, having a flexible and reliable framework to prototype and verify these new methodologies minimizes the development cycle time. This Ph.D. Thesis considers these problems and 
proposes novel solutions for each of them.

The chapter is organized as follows: We present the motivations for this Ph.D. Thesis in Section 1.1. Next, in Section 1.2 we list the contributions of this Ph.D. Thesis as well as the publications it has originated. Finally, we outline the structure of the rest of the book in Section 1.3.

\subsection{Motivation}

Fixed-point numbers are defined by means of two variables: the width, in number of bits, of the number representation (without counting the sign bit) and the position of the binary point in that representation. They are a powerful tool if we need to achieve a high computation speed, as they are operated in the same way as integer numbers. ${ }^{1}$ But the format imposes very strict limits on the accuracy it can achieve when representing fractional numbers: A fixed-point number with the binary point in bit 2 (i.e. only bits 0 and 1 are on the right-hand side of the point) will only be able to represent numbers in increments of 0.25 . In general, as a consequence of this lack of accuracy, fixed-point numbers deviate from the fractional magnitude they represent. We refer to the difference between the original magnitude and the fixed-point value as its quantization noise.

Studying the effects of quantization noise is of critical importance when transforming a model with infinite numerical precision into a digital circuit using fixed-point arithmetic. In order to keep the quantization noise at the output of the circuit within reasonable limits, designers have to evaluate the propagation of the noise and determine the wordlength assignments to all the variables in the system. Otherwise, the divergence between the ideal model and the circuit could be too large and the implementation would deem as invalid. Sensible designers will try to obtain optimized word-length assignments that minimize the size of the circuit while keeping the quantization noise in compliance with the accuracy constraints required by the design.

Figure 1.1 outlines the fixed-point Word-Length Optimization (WLO) process. We can identify three major areas of interest in the flow: i) Determining the dynamic range of the signals in the system in order to allocate the integer word-length of each variable, ii) assigning a certain number of bits to the fractional word-length and iii) obtaining the quantization noise to compare it with the design constraint. These last two tasks are typically carried out iteratively in an interleaved manner. None of these three areas is trivial, and each of them is a large field of research on its own.

When applying the quantization to a number, two different methods can be applied to determine how to approximate the resulting value: truncation or rounding. In the former, the digits out of the assigned fractional size are removed and the rest of the number is left as it is. For example, number 3.53194 truncated to three decimals would be 3.531. Rounding, on the other hand, approximates the remaining number to its closest value

\footnotetext{
${ }^{1}$ In fact, integer numbers can be considered a particular case of fixed-point number where the binary point is after the Least Significant Bit.
} 


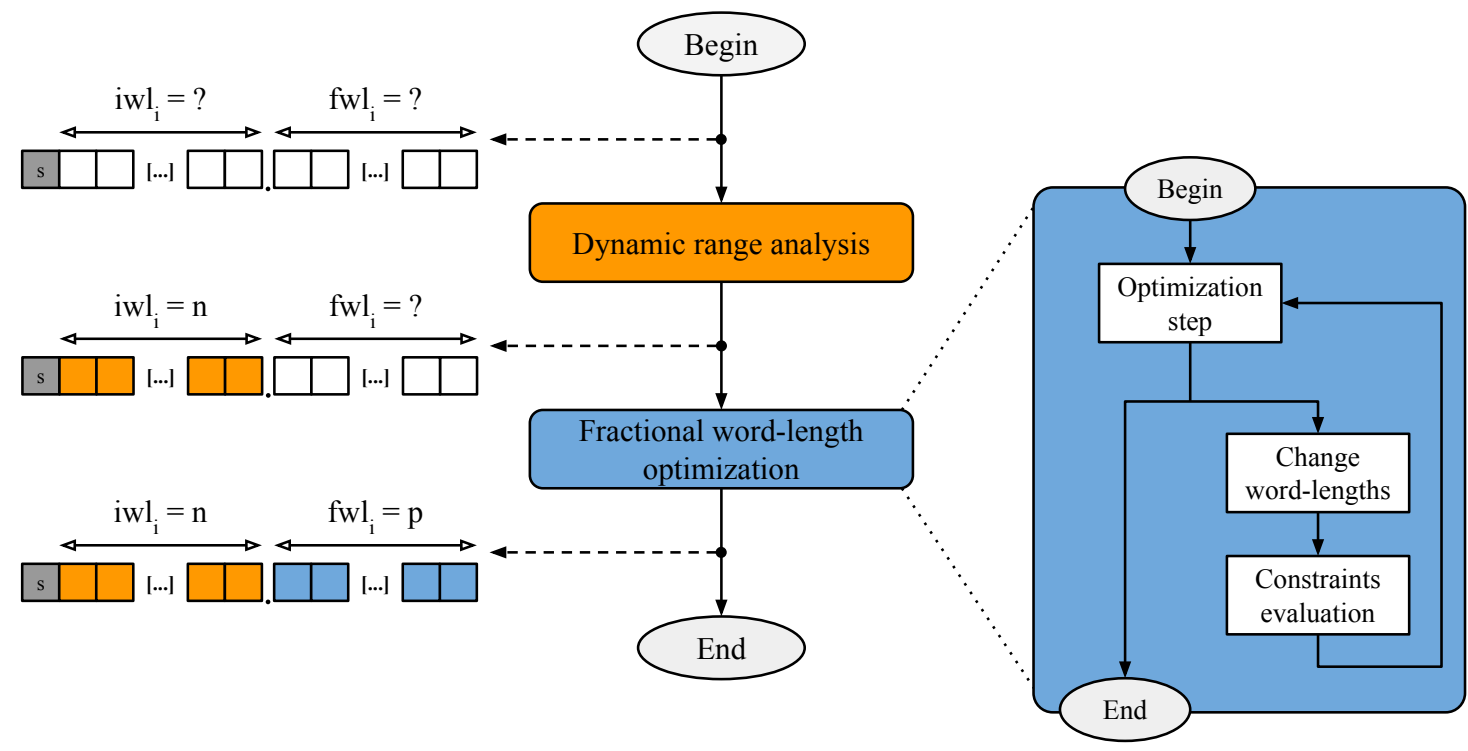

Fig. 1.1: Fixed-point word-length optimization flow.

before discarding the digits that do not fit. The previous example, if instead of truncated was rounded, would be 3.532. In this Ph.D. Thesis we will always apply rounding, and the numbers will be represented, as it can be seen in Figure 1.1, using signed magnitude representation.

Simulation-based techniques for modelling the dynamic range and the round-off noise are the most reliable and inclusive approaches, but also the slowest. In order to obtain an accurate model, usually large input data sets are required. That makes simulationbased methods impractical for WLO, as estimations have to be made many times with different combination of word-lengths as the optimization progresses. To overcome this, several techniques have appeared over the years in order to replace the simulation-based techniques with analytical or semi-analytical methods. These approaches are several orders of magnitude faster than simulation-based ones, but they are limited on the properties of the systems that they can represent accurately.

The Departamento de Ingeniería Electrónica ${ }^{2}$ (IE) of the Universidad Politécnica de Madrid $^{3}$ (UPM) has maintained, for more than ten years now, a continuous line of research on the effects of quantization of digital systems applying intervals and their extensions. This research has produced a large body of work that exhaustively covers different types of systems, from Linear Time-Invariant (LTI) to strongly non-linear ones. This Ph.D. Thesis has used that previous work as the foundation for developing a novel methodology capable of modelling systems that contain control-flow structures. In practice, this breakthrough largely broadens the range of systems that can be studied using intervals, as many real-world applications contain this type of structures.

The other area of interest, the assignment of bits to the fractional word-lengths,

\footnotetext{
${ }^{2}$ Department of Electronic Engineering

${ }^{3}$ Technical University of Madrid
} 
is a non-convex, NP-complete problem [CW02] commonly driven by a search algorithm responsible for finding the best combination of word-lengths that complies with the constraints (which may not be just a function of the quantization noise, but also of the area, performance or power consumption) of the circuit under analysis. The search algorithms need to be able to traverse the solution space (i.e. all the possible combinations of wordlengths), which can become extremely large if the circuit to optimize has many quantized elements, and find an optimized and valid combination of word-lengths. Simulation-based optimization methods are still the most general and reliable approaches and, as such, designers may choose them over analytical-based methodologies. Because of this, it is still important to develop fast simulation-based optimization techniques that minimize the execution time while granting quality results.

We must take into account that WLO is evolving constantly along with the demands of the technology and the industry. Also, it is not an isolated process. As shown in several works $\left[\mathrm{CCC}^{+} 06, \mathrm{Caf08}\right]$, it is highly coupled with other fundamental tasks in general EDA flows. Because of this, researchers and developers need tools that offer them the possibility of prototyping, verifying and integrating new WLO methodologies with minimal effort.

Considering all of the above, the main ideas that encouraged this Ph.D. Thesis are:

- Analytical models for modelling dynamic range and round-off noise are far from being as inclusive as the reliable, but impractical for real life applications, simulationbased techniques.

- There is plenty of room for developing new word-length optimization algorithms, given the properties of the problem to solve.

- To the best of our knowledge, none of the existing automated quantization frameworks in the academic environment met our criteria in terms of flexibility and integration capabilities.

\subsection{Thesis contributions}

Among the different distortions arising in fixed-point implementations, the RoundOff Noise (RON) is widespread considered the most important one. The RON is defined as the error due to the quantization of the internal operations of the system and it is, along with its relation to the value of the unquantized signal (Signal to Quantization Noise Ratio, SQNR), the most commonly used metric to constraint the deviation of a fixed-point implementation with respect to the ideal model with infinite precision. This $\mathrm{Ph} . \mathrm{D}$. Thesis focuses on the RON, while other distortions such as coefficient sensitivity or limit cycles are left out of the scope of this book. We address a number of key topics that we have considered fundamental to achieve reliable and efficient solutions to the WLO problem. In particular, the main contributions of this Ph.D. Thesis are: 
- An in-depth review of the state of the art of word-length optimization, identifying outstanding problems and potential areas for improvement (Chapter 2).

- A comprehensive methodology based on ME-gPC and extensions of Interval Arithmetic for modelling the dynamic range (Chapter 3.2) and round-off noise (Chapter 3.3) of non-linear systems with control-flow structures.

- A technique for clustered noise injection to deal effectively with the scalability issues of polynomial-based models as the size of the target applications increases (Chapter 3.4).

- Three novel simulation-based optimization algorithms for solving the fixed-point word-length bit allocation problem in reasonable times (Chapter 5).

- HOPLITE, a modular, automated quantization framework to allow fast prototyping and verification of new techniques for system modelling and word-length optimization, that offers common interfaces for previous and forthcoming quantization methodologies, provided under a free software license (Chapter 6).

\section{List of Publications}

We have presented some of the contributions of this Ph.D. Thesis, as well as other related research carried out, at different international peer reviewed conferences and journals. Here we list those publications in reverse chronological order.

\section{Journal papers}

- E. Sedano, J.A. López, C. Carreras, "Scalable Modelling of the Fixed-point Round-off Noise through Clustered Noise Injection", submitted to Computer-Aided Design of Integrated Circuits and Systems, IEEE Transactions on.

- E. Sedano, C. Carreras, J.A. López, "Acceleration of simulation-based techniques for fixed-point word-length selection in DSP systems" submitted to IET Circuits, Devices $\mathcal{G}$ Systems.

- E. Sedano, J.A. López, C. Carreras "Dynamic range and round-off noise estimation of fixed-point systems with control-flow structures", to be submitted to Cirtuits and Systems-I, IEEE Transactions on.

- L. Esteban, J.A. López, E. Sedano, S. Hernández-Montero, M. Sánchez, "Quantization Analysis of the Infrared Interferometer of the TJ-II Stellarator for its Optimized FPGA-Based Implementation", Nuclear Science, IEEE Transactions on (Volume 60, Issue 5). Page(s): 3592-3596. Impact factor: 1.219 


\section{Conference papers}

- E. Sedano, J.A. López, D. Menard, "Automated Data Flow Graph partitioning for a hierarchical approach to wordlength optimization", 10th International Symposium on Applied Reconfigurable Computing, ARC 2014, Vilamoura (Algarve, Portugal), April 14 - 16, 2014

- L. Esteban, J.A. López, E. Sedano, M. Sánchez, "Quantization Analysis of the Infrared Interferometer of the TJ-II for its Optimized FPGA-based Implementation", Proceedings of IEEE 18th Real Time Conference, RTC'12, Berkeley (California, USA), June 11-15, 2012.

- E. Sedano, Juan A. López, C. Carreras, "Acceleration of Monte-Carlo simulationbased quantization of DSP systems", International Conference on Systems, Signals and Image Processing 2012, IWSSIP 2012. Vienna (Austria), April 11-13, 2012.

- E. Sedano, Juan A. López, C. Carreras, "A Fast Interpolative Wordlength Optimization Method for DSP Systems", VIII Southern Programmable Logic Conference, SPL 2012, Bento Gonçalves (Brasil), March 20-23, 2012. Page(s): 51 56.

\section{Book chapters}

- J.A. López, E. Sedano, L. Esteban, G. Caffarena, A. Fernández-Herrero, C. Carreras. "Applications of Interval-Based Simulations to the Analysis and Design of Digital LTI Systems", in Applications of Digital Signal Processing, ISBN 978-953307-406-1, InTech, November 2011.

\subsection{Book structure}

The Ph.D. Thesis is organized as follows: Chapter 2 overviews the state of the art of the quantization field of research. The chapter reviews the main body of work in analytical dynamic range and fractional precision modelling, the diverse approaches to word-length optimization, the existing literature addressing the pressing matter of computational scalability, and briefly describes several existing frameworks for quantization analysis and fixed-point optimization.

In Chapter 3 we approach the problem of modelling the dynamic range and RON in fixed-point implementations of digital systems. After introducing the theoretical foundations of extensions of IA and ME-gPC expansions, we present a novel method based on ME-gPC for accurately computing the dynamic range and round-off noise in complex systems that contain control-flow structures. To do that, we propose a technique to determine automatically the parts of the domain that generate different results in the decision operators associated to each control-flow structure and model each of them 
independently. Then, we compute the system statistics from the partial results. In this section we also cover the issue of scalability presenting an iterative methodology to introduce round-off noise sources in small, manageable groups, and we show a guided partitioning algorithm to minimize the impact of the clustered noise injection in the accuracy of the results. We prove the validity of these techniques in Chapter 4 through several case studies.

In Chapter 5 we introduce three new greedy search techniques for optimizing the word-lengths of the variables in fixed-point implementations. These techniques consider different approaches for reducing the number of required Monte-Carlo simulations. The first one relies on minimizing the number of different configurations to evaluate, the second one takes advantage of imprecise estimations in the initial stages in order to accelerate the evaluation of each different configuration and the third one combines the other two approaches.

Chapter 6 describes HOPLITE, a modular framework for word-length optimization, detailing the work flow, the interfaces of each module and showing how to extend it with new features and functionalities.

In Chapter 7 we draw the conclusions and outline future lines of research. Finally, Appendix A presents a technique to speed up the computation and reduce the memory requirements of the $\mathrm{C}$ matrix, a key element in the propagation of $\mathrm{PCE}$ coefficients through strongly non-linear operations. 


\title{
Chapter 2
}

\section{Dynamic range and round-off noise estimation}

\author{
Those who assume hypotheses as first princi- \\ ples of their speculations... may indeed form \\ an ingenious romance, but a romance it will \\ still be.
}

Roger Cotes, Preface to Sir Isaac Newton's Principia Mathematica

Quantization noise, its effects on the performance of digital systems, and the WLO problem have been a concern for digital designers since the 70's. Back then, when computers were slow and memory was an expensive resource, using fixed-point arithmetic for the implementation of digital systems was the norm. This cheaper, faster numerical representation was used to allow computing complex numerical systems in reasonable times using limited resources. Even though the landscape of computing has quickly evolved and the old constrains have been overtaken, requisites such as low silicon area and power consumption or high circuit speed still apply and motivate the use of fixed-point in numerous application niches where it remains the dominant type of arithmetic.

Because of all this, quantization noise and WLO have attracted the interest of many researchers for several years now. This complex problem -the conversion of descriptions of infinite numeric precision into refined fixed-point implementations- comprises several different non-trivial aspects that have evolved into whole research fields on their own. The main issues involved in of the quantization process have been already depicted in Figure 1.1. Given the magnitude of each of these sub-tasks, it is not feasible to consider all of them together. In practice, each sub-problem must be addressed individually and its results combined with those of the other sub-problems.

This chapter overviews the most relevant proposed methods that aim to solve each of the sub-problems posed by fixed-point optimization. It shows the wide variety of existing 
techniques, approaches and even optimization objectives that have been considered and motivates the need for the advanced techniques and modular infrastructure presented in this Ph.D. Thesis. Section 2.1 overviews the different simulation-based and analytical methods that are used to model the dynamic range (Section 2.1.1) and fractional precision (Section 2.1.2) of digital systems. In Section 2.2 we compile numerous optimization techniques and their characteristics. One of the currently most pressing issues in WLO, the computational scalability of the approaches, is outlined in Section 2.3. Finally, in Section 2.4 we summarize several academic fixed-point quantization frameworks.

\subsection{Dynamic range and fractional precision modelling}

Modelling how the output of a given fixed-point implementation deviates from the ideal result is essential for any quantization process. If such model is not capable of capturing the effect of limiting the possible values for both integer and fractional values, every optimization effort will be rendered worthless since it will be based on misleading data.

The most reliable, but also the slowest way of modelling the propagation of values through a system with and without quantized operators is using Monte-Carlo Sampling (MCS). The technique relies on feeding very large sets of random inputs following the expected input distributions to a bit-true simulation of the fixed-point application to obtain numerical results from which the statistical distributions of the signals are extracted. The main advantage of this approach is that every type of application, regardless its complexity, is supported. Although traditionally it is extensively used in the fixedpoint optimization literature [SK95, SB04a, RB05], this method has fallen into disuse because of the long simulation times it requires [DCALP98]: To consider a MCS run reliable, i.e. mean values of the signals within a given confidence interval $\alpha$, the number of required random input samples must be determined from such confidence interval. As discussed in [Jer84] and [ZB97], when the noise constraint is of order $10^{-k}$, being $k$ an integer value $k \geq 0, N=10^{k+1}$ samples need to be used. This is a heavy burden for the WLO process, given the large number of simulations required, one for each set of finite word-lengths being considered.

To overcome this problem, several analytical, semi-analytical and hybrid approaches have been proposed. These methodologies intend to compute a mathematical model of the fixed-point accuracy metrics depending on the word-lengths of the different elements inside the system. The main advantage of this type of approaches is that it achieves huge time savings with respect to MCS: Even though the computation of the model is often slower than a simulation of $N$ samples, the analysis has to be done only once, while the simulations have to be re-run for each new combination of word-lengths (also known as Word-Length Vector, WLV). But analytical models have limited applicability: for the model to produce accurate results, the systems have to comply with specific constraints.

This section overviews several analytical and semi-analytical approaches to system 
modelling when representation constraints are applied to the integer and fractional parts of the numeric values. First we review techniques for the estimation of dynamic ranges in Section 2.1.1, and then those for modelling fractional precisions in Section 2.1.2.

\subsubsection{Dynamic range estimation}

As shown in Figure 1.1, commonly the first step of the WLO flow is to allocate the number of bits that the integer part of the fixed-point number will require. This process is known as dynamic range estimation and it is crucial to prevent overflow errors that could lead to very large deviations from the expected results. Interval Arithmetic (IA) [Moo66] has been initially used to estimate the dynamic range. This technique is able to obtain the range of all the possible results of a given function, but it has an important drawback: It does not capture data correlations so it tends to overestimate the output ranges, thus generating inaccurate and conservative results, even occasionally becoming useless for practical purposes [MB03]. A very popular, but also conservative, method to estimate dynamic ranges is the $L_{p}$ norm, proposed in [Jac70] and applied in [CVKF03]. It uses the system transfer function for its calculations, which makes it inapplicable to non-linear systems.

Affine Arithmetic (AA), first introduced by [SDF97], is proposed as an improved method to overcome IA limitations in [FRPC03, LCCNT04, Ló04]. This method captures first order correlations between signals, but it is not capable of retaining temporal correlations or accurately propagating non-linear terms. Thus, AA is suitable for estimating the range of LTI systems but, other than that, it has a limited applicability range. There are several works that extend that range. In [LCNT07] the authors propose a method to extend AA to recursive systems by adding AA markers to each quantized signal. Then, the input signal range is split in multiple intervals, which are propagated with IA, and finally the results are merged.

The noise source and propagation detailed in [LCCNT08] is as precise as numerical methods. But introducing uncertainties conservatively, like additional terms due to round-off, causes loss of precision in the obtained results, which makes then invalid.

AA has proven to provide the fastest results for this type of systems, but still fails to capture correlations due to non-linear operations. This is partially solved by MAA, presented in [SLMW03, SLMW04], that allows to capture correlations when multiplying two affine forms by taking into account all high order terms. Nevertheless, MAA monomials are not orthonormal. This implies that output gains are nor directly transferable if calculations are to be repeated with different values.

In [WZN04b, WZN04a], a solution based on Karhunen-Lòeve Expansion (KLE) and Polynomial Chaos Expansion (PCE) is proposed to estimate the dynamic ranges of the signals in non-linear systems. The input signals are decomposed into a set of polynomials that are orthogonal with respect to the measure of the Gaussian random variable. Once the signals are decomposed, they are easily propagated through the non-linear system 
preserving the correlations between them. The statistics of all the signals are also easily obtained. However, the application of PCE is restricted to the estimation of the dynamic ranges using Hermite polynomials as the orthogonal basis. The work in [Est11] combines MAA with PCE to overcome the previous shortcomings. Also, this work introduces the use of Legendre polynomials instead of the Hermite ones to perform the computations. Other approaches based on KLE can be found in [ÖNG08] and [BCMM11]. But all these techniques still fail to model systems accurately in the presence of strong non-linearities like those found in enclosure operations (min, max, floor, ceiling) or in conditional constructs.

Section 3.1 details the mathematical processes of AA, MAA, PCE and ME-gPC.

None of the previous works considers control-flow structures such as conditional branches and loops that cannot be solved statically. These constructs are extremely common in real-life applications, and the range of systems that can benefit from approaches that do not consider them is severely limited. It is possible to find several works in the literature that consider the effects of fractional bit-widths in systems with control-flow structures but, to the best of our knowledge, only the work in [WZN06] points at this issue and [Wu12] addresses it for the accurate estimation of dynamic ranges. It extends PCE to consider the conditional probability of each possible execution path, and propagates the polynomials alongside with them. It defines the propagation flow through each type of control-flow structure and formulates an algorithm to treat the system and propagate the PCE through those constructs. Scalability may prove an issue when several Random Variables (RV) are considered, given the number of terms and probabilities to track.

\subsubsection{Fractional precision analysis}

While dynamic range estimation tries to establish the required number of bits to avoid overflow errors in the system, the objective of fractional precision analysis is to estimate the numerical error introduced by the selected Fractional Word-Length (FWL) of each variable. Consequently, the analytical approaches to this process will usually return not a single value, but an expression function of the FWL of all the variables to quantize.

Most of the methodologies used to estimate the dynamic range have also been extended to the analysis of fractional precision. Such is the case of AA, used for this purpose in [FRC03, PH06, LGML05, CCLF10, LCCNT08], and MAA/PCE, applied in [Est11] to evaluate the round-off noise in nuclear fusion interferometry systems. A different type of arithmetic derived from IA known as General Interval Arithmetic (GIA) is developed in [Han75] and applied to fixed-point modelling in [CGL ${ }^{+}$09]. It intends to deal with the pessimistic results of IA. The approach is similar to AA, but using intervals instead of scalars as coefficients. This means using interval additions, subtractions and products, and the results of such operations are guaranteed to be in GIA form. This approach is 
Table 2.1: Techniques for the evaluation of the Dynamic Range (DR) and RON.

\begin{tabular}{|c|c|c|c|c|c|}
\hline Technique & Features & Systems & DR & RON & References \\
\hline AA & $\begin{array}{l}\text { Captures first order } \\
\text { correlations } \\
\text { between signals. }\end{array}$ & LTI & Yes & Yes & $\begin{array}{c}\text { [FRPC03], [LCCNT04], } \\
\text { [FRC03], [Ló04], } \\
\text { [LGML05], [LCNT07] }\end{array}$ \\
\hline MAA & $\begin{array}{l}\text { Captures second order } \\
\text { correlations for } \\
\text { non-linear multiplications. }\end{array}$ & $\begin{array}{l}\text { LTI } \\
\text { and } \\
\text { smooth }\end{array}$ & Yes & Yes & $\begin{array}{c}\text { [SLMW03], } \\
\text { [SLMW04] } \\
\text { [CCLF10] }\end{array}$ \\
\hline PCE & $\begin{array}{c}\text { Gives accurate results in } \\
\text { strongly non-linear systems. }\end{array}$ & Polynomial & Yes & Yes & $\begin{array}{c}\text { [WZN04b], [WZN04a], } \\
\text { [WZN06], [Est11] }\end{array}$ \\
\hline $\begin{array}{c}\text { PCE } \\
\text { control-flow }\end{array}$ & $\begin{array}{c}\text { Capable of modelling } \\
\text { control-flow structures } \\
\text { (conditional probabilities). }\end{array}$ & $\begin{array}{l}\text { Polynomial w. } \\
\text { control-flow } \\
\text { structures }\end{array}$ & Yes & No & [WZN06], [Wu12] \\
\hline ME-gPC & $\begin{array}{l}\text { Improves accuracy } \\
\text { of PCE in systems } \\
\text { with discontinuities. }\end{array}$ & Polynomial & Yes & No & [WK05] \\
\hline GIA & $\begin{array}{l}\text { Uses intervals instead of } \\
\text { scalars as coefficients. } \\
\text { More scalable than AA. }\end{array}$ & Smooth & Yes & Yes & {$[\operatorname{Han} 75],\left[\mathrm{CGL}^{+} 09\right]$} \\
\hline $\mathrm{PT}$ & $\begin{array}{l}\text { Applies Taylor developments } \\
\text { to each operator and takes } \\
\text { the two first moments. }\end{array}$ & Smooth & Yes & Yes & [SB04c] \\
\hline $\mathrm{AD}$ & $\begin{array}{l}\text { Models the noise using } \\
\text { Taylorian approximations. }\end{array}$ & Smooth & Yes & Yes & [WP98], [GML04] \\
\hline SNA & $\begin{array}{c}\text { Takes into account } \\
\text { PDFs of noise symbols. }\end{array}$ & Polynomial & Yes & Yes & [AZ07], [AZ08] \\
\hline SNSM & $\begin{array}{l}\text { Models subsystems } \\
\text { independently in a } \\
\text { hierarchical way. }\end{array}$ & Polynomial & Yes & Yes & {$[\operatorname{Par} 12]$} \\
\hline AT & $\begin{array}{l}\text { Linearly independent } \\
\text { functions interact with } \\
\text { the arithmetic spectrum. }\end{array}$ & Polynomial & Yes & Yes & [ZR09], [PRZ10] \\
\hline
\end{tabular}

more scalable for systems rich in non-linear operations, but it is often more pessimistic than AA.

Several other analytical estimation mechanisms have been developed over the years. One of them studies the effects of quantization by means of Perturbation Theory (PT), as proposed in [SB04c]. This approach assumes quantization noise as a small deviation from the infinite precision signal. In order to obtain the finite precision output expres- 
sion, a second order Taylor development is applied to each operator, and then the first and second order moments of the output quantization noise are computed. Another type of approach is found in [WP98, GML04], where Automatic Differentiation is used to compute the sensitivities of the outputs to the different fractional word-length combinations, using Taylorian approximations to model the shape and propagation of the noise. This type of technique allows the comparison of floating-point and fixed-point implementations, but it cannot be applied to non-differentiable specifications.

In turn, [AZ08] introduces Symbolic Noise Analysis (SNA). This approach takes into account the PDFs of the different noise symbols, so there is no limitation on the type of PDFs to be used by the method. As a result of this, the error model in each sub-block of the system can be replaced by models of its outputs generated by other means such as simulation.

The Single Noise Source Model (SNSM) presented in [Par12] studies the system as a hierarchical decomposition of sub-blocks. The approach studies each of the sub-blocks independently, computing the PDF and the Noise Power Spectral Density, and they are used to computed the shape of the noise, which is added to the output of the block. The process carries on until the whole system has been modelled.

Authors in [PRZ10] propose Arithmetic Transforms (AT) [ZR09] to evaluate the error introduced by quantization in arithmetic systems modelled as Taylor series or multivariate polynomials. In AT, functions are expressed as a set of linearly independent functions, that interact with the arithmetic spectrum, a set of coefficients each of which multiplies a function. Several different methods (such as Matrix transform, interpolation construction or expression rewriting) exist to obtain the AT for a function.

We summarize the techniques reviewed in Sections 2.1.1 and 2.1.2 in Table 2.1. There we can identify several relevant research groups that have developed different research lines on analytical methodologies. We identify some of them:

- J.A. López, C. Carreras, G. Caffarena et al. (UPM, Madrid, Spain) have developed automated interval analysis, probabilistic intervals and extensions of AA such as $\mathrm{MAA}$ and MAA/PCE.

- D. Ménard, O. Sentieys, R. Rocher et al. (IETR-INSA, Rennes and Lannion, France) have focused their research on analytical expressions, lately developing the Single Noise Source model.

- G. Constantinides, D. Boland, P.Cheung et al. (Imperial College, London, UK) have used ASA, guaranteed bounds and Handelman representations for their analysis.

- K. Radecka, O. Sarbishei, Z. Zilic et al. (McGill University, Montreal, Canada) have consistently applied Arithmetic Transformations to model polynomial systems.

- B. Wu, J. Zhu, F.N. Najm et al. (University of Toronto, Toronto, Canada) have studied the dynamic range of systems through PCE and its extensions, but without taking the analysis of the RON into consideration. 


\subsection{Word-length optimization}

The main purpose of quantization is to make possible the implementation of mathematical algorithms while dealing with the trade-off between implementation cost and numerical accuracy. To this end, the optimization process is a key stage in the quantization process. Over the years this process has experienced an important evolution from the fast but far from optimal Uniform Word-length Optimization (UWL), where only one degree of freedom is allowed and all the variables share the same size, to a variety of cost-effective Multiple Word-length Optimization (MWL) algorithms. These approaches allow as many degrees of freedom as variables are in the system at the cost of longer execution times. Different heuristics have been proposed to reduce the duration of the process.

In this section, we first present a review of classical and state-of-the-art search-based WLO algorithms. Then we describe alternative mathematical formulations of the optimization process, and finally we overview different cost functions and mixed approaches, where the WLO is carried out along with other design tasks.

\subsubsection{Search-based optimization techniques}

Being a non-convex, NP-hard problem [CW02], finding the optimal combination of word-lengths for the fixed-point implementation of a given system is not a trivial problem. In the past the same word-length was assigned to all the variables in the system. This is known as UWL paradigm. But since the obtained solution is far from optimal, a much more ambitious objective is addressed: The MWL paradigm, where each variable has its own word-length. This paradigm requires the development of highly-efficient algorithms in order to find optimized solutions in reasonable times.

This section overviews the different search and optimization algorithms for WLO that have been proposed over the years. Due to the scope of this Ph.D. Thesis, only fractional word-length optimization methods are reviewed. Nevertheless, there are works [CCL03a] focused on the optimization of the integer part of the fixed-point representation, also known as scaling.

\section{Optimal searches}

The trivial approach to find the optimal solution to the WLO problem is to evaluate every possible word-length combination in the system and choose the one that minimizes the cost function. This methodology, known as complete search [SK94, CSPL01], is impractical due to obvious reasons: The solution space grows exponentially with the number of signals in the systems [HE06]. Thus, the approach is infeasible for real applications.

Notwithstanding the above, some works have suggested the application of this technique delimiting the solution space by means of establishing lower and upper bounds in 
the minimum word-length set [SK94, CB94] and minimum uniform word-length [CB94], respectively. But as shown in [CCL03b], it is possible that the optimal solution is not found within those bounds, given the properties of the WLO problem.

A different approach is presented in [CCL03b], where the WLO of LTI algorithms is formulated in terms of Mixed Integer Linear Programming (MILP). A system of equations and inequations that represents the algorithm to analyse, the cost function to minimize, the error function and the error constraint is built and solved through an automated solver. This approach is prone to suffer an excessive increment in the number of variables in the formulation. Since the noise power expression is exponential, as many variables as bits are permitted in the solution space are introduced. Consequently, even though further simplifications are introduced in order to reduce the complexity of the formulations, the solving process can take several days or even weeks to find the solution for medium sized problems.

\section{Exhaustive and stochastic searches}

In an attempt to reduce the number of word-length combinations to evaluate without renouncing to obtain optimal or near-optimal solutions, different exhaustive search algorithms are proposed. In the method presented in [SK95], a Minimal Word-length Solution (mWS) is first obtained by calculating the smallest word-length for each signal that makes the output noise comply with the constraint while the rest of the system is represented with arbitrarily high floating-point precision. Afterwards, the solution is iteratively refined until the design constraints are met by testing all possible combinations of the distribution of $b$ bits among the system variables. The mayor drawback of this approach is that, since it stops as soon as a valid solution is found, it easily gets stuck in local minima. Alternatively, [CB94] presents a branch-and-bound technique that computes the solution between the mWS and a maximum word-length solution. This approach is later extended in [MCS06].

Simulated Annealing (SA) is a generic probabilistic algorithm that has widely demonstrated an excellent performance in optimization problems. It iteratively evaluates the neighbours of the current solutions and decides, with a probability $p$, if the solution moves to the evaluated neighbour. If the solution is improved, $p=1$. Otherwise, $p=k$, where $k$ is a value that is set to 1 at the beginning of the algorithm execution and is gradually reduced in each iteration according to a given cooling scheme. SA was first applied to the WLO problem by [CDMV88]. In this early work, the WLO is formulated as a MILP problem, and SA is used to obtain the solution. The cost of each configuration is computed in terms of error power and implementation cost, and it is calculated for each word-length configuration considered in each iteration. The SA approach is also applied in $\left[\mathrm{LGC}^{+} 06\right]$ and [Caf08], although the technique entails long execution times that limit its applicability.

Some works [NTM92, NLC ${ }^{+} 96$, SA05, AZ06] have proposed the use of Genetic Al- 
gorithms (GA) to find suitable word-length combinations. In GA, several candidate solutions have a set of properties that are modified and combined (mutated) in an attempt to mimic the evolution process. Typically, the search starts from a random set of individuals that are iteratively mutated. The new set of individuals after a mutation iteration is called a generation. The best solutions are stochastically selected from each generation and mutated to form the next one. The process finishes after a pre-established number of generations or once a number of fit solutions have been found. In [NTM92] the GA is directly applied to optimize the implementation of a LMS filter, while in other works it is combined with other heuristics in order to improve its performance. The authors in $\left[\mathrm{NLC}^{+} 96\right]$ combine GA and a gradient based heuristic approach in order to reduce the computation time. The multi-objective GA variant is explored in [SA05, AZ06], where multiple cost functions are optimized at once.

\section{Greedy searches}

Given the excessive execution times of the algorithms presented in the previous sections, a number of greedy heuristics have been proposed in an attempt to trade execution time with quality of results, improving the former without incurring in excessive penalty in the latter. On this behalf, [CSL02] summarizes a number of sequential searches based on different greedy approaches for obtaining an optimized combination of word-lengths. These algorithms are notably faster than the ones previously reviewed, but they have a greater tendency to get stuck in local minima.

The max-1 procedure computes a suitable Minimal Uniform Word-Length Vector (mUWL) and, after that, the variables iteratively compete for losing one bit while specifications are met. The signal that produces the minimum cost increment is the one selected each time. In [CSPL01, Caf08] some variations for this algorithm are proposed in an attempt to reduce the chance of getting stuck in local minima. The authors in [HEKC01, SK95] propose a scheme for the $m i n+1$ algorithm. Here, a mWS is computed in the first place. Then, all the variables compete for increasing one bit. The signal that produces the maximum noise decrement is selected, and the process is repeated until specifications are met. A generalization of this scheme is presented in [CSL02] under the name of $m i n+b$. In this case a budget of $b>1$ bits is distributed among the signals.

The hybrid procedure [CSL02] performs a min +1 search and uses its results as the starting point for a max-1 search. The heuristic search [HEKC01, SK95] starts with a minimum solution and increases all system variables' word-lengths equally until the specifications are met. The evolutive search [CSPL01] begins with an unquantized system and finds the minimum word-length for a single variable. That signal word-length is set to that value plus one, and the process is repeated for another variable until all of them have word-lengths assigned.

The preplanned search [HEKC01] performs an initial phase in which the sensitivity of every signal word-length is obtained. After that, the mWS is found and the search is 
guided by the derivative of the independent performance of each variable, increasing the word-length of the variable with steepest gradient of performance. This method reduces notably the number of simulations in contrast to other sequential searches.

In [NMS11] the authors propose a Greedy Randomized Adaptive Search Procedure (GRASP) for solving the WLO problem. It consists of two different stages that are executed iteratively: first, a randomized search aimed at avoiding local minima is used to find a sub-optimal solution. Then, a local search is used to refine the solution obtained in the previous stage. In this second stage, a steepest-descent-mildest-ascent, or Tabu Search, is applied. Contrary to other greedy algorithms, which are mono-directional (either steepest-descent or mildest-ascent), in this case movements in both directions are allowed. The Tabu search calculates the gradient for each operator and then selects the new partial solution according to the direction of the search. Once the noise constraint is surpassed, the direction of the search is reversed. To avoid loops, a set of tabu operators is used.

\subsubsection{Alternative optimization approaches}

Despite the reduction of the execution time achieved by some of the techniques presented in the previous section, conversion and optimization are still an issue when large applications have to be implemented in fixed-point arithmetic. In these cases, different approaches must be followed. In this section two main strategies are presented: On the one hand, the formulation of the WLO as a mathematical problem and, on the other hand, the reduction of the solution space by means of signal grouping or partitioning in smaller, treatable problems.

\section{Mathematical formulations}

Apart from the MILP approach presented in Section 2.2.1, which has the problem of excessively long execution times when the size of the problem grows, other mathematical formulations for the WLO problem have been developed over the years. An analytical approach based on Lagrange Multipliers is proposed in [CT05, FL99]. The method, also referred to as marginal analysis, proposes an algorithm that resembles to previously reviewed steepest descent ones, but assuming three simplifications: The word-lengths are real numbers, the cost is a linear combination of the word-lengths, and the quantization noise only depends on the final word-length. This method is improved in [CT07], where the WLO problem is transformed into a Geometric Programming problem.

Another approach to speed up optimization analytically is presented in $\left[\mathrm{HNK}^{+} 04\right]$, where IA is used to estimate the error. By using the summation of word-lengths as complexity measurement, the objective function is linear and Linear Programming can be applied to optimize the system. A similar approach is developed in [Fio08], where a Pareto-optimal curve is used for the optimization of cost and performance. A quadratic model is assumed for multipliers, while adders use a linear one, and a non-linear iterative 
Table 2.2: Classification of optimization techniques.

\begin{tabular}{|c|c|c|}
\hline \multirow{1}{*}{ Search-based } & Optimal & $\begin{array}{c}\text { Complete search } \\
\text { MILP }\end{array}$ \\
\cline { 2 - 3 } & Exhaustive & $\begin{array}{c}\text { Branch and Bound } \\
\text { Simulated Annealing } \\
\text { Genetic Algorithms }\end{array}$ \\
\cline { 2 - 3 } & & max-1 \\
Greedy +1 & Hybrid \\
& & Evolutive \\
Mathematical formulations & \multicolumn{2}{|c|}{$\begin{array}{c}\text { Preplanned } \\
\text { GRASP }\end{array}$} \\
& \multicolumn{2}{|c|}{ Leometric Programming } \\
& \multicolumn{2}{|c|}{ Linear Programming } \\
\hline
\end{tabular}

algorithm is proposed to obtain the combination of word-lengths that optimizes the implementation cost.

Finally, [CCC09] proposes Sequential Quadratic Programming to optimize power consumption. An algorithm based on sensitivity analysis to constrain the number of possible word-lengths combinations and a simple noise correlation model for signal forks is applied. By doing this, and by using the noise model described in [CCL99], the convexity of the solution space is guaranteed, thus greatly simplifying its exploration.

Table 2.2 shows a hierarchical classification of the optimization techniques presented in this section and Section 2.2.1.

\section{Grouping and partitioning}

The main problem of the MWL paradigm is that, as the number of variables grows, the solution space becomes too large and thus excessively expensive to explore. In order to maintain the number of dimensions within reasonable bounds, signal grouping and system partitioning have been proposed.

Clustering signals into groups is a compromise solution between the UWL and the MWL paradigms in which the same word-length is applied to every signal in each cluster [FCR02]. On this behalf, different clustering strategies have been proposed. A possible alternative is to cluster signals according to the type of operator they are input or output of. That is the approach suggested in [SK94, SK95], where adders, multiplexers and delays are treated in this way.

Signal clustering has been typically applied when additional architectural issues were considered in the same stage of the HLS design cycle. This is the case of [HMS07], where 
resource sharing is considered. In this case, those operations that will be mapped over the same hardware resources are quantized with the same word-length. By doing this, the solution space to explore is reduced and the hardware resources are made the most of. Signal clustering in combined HLS and WLO is also explored in [KS01, Caf08].

Partitioning large designs and studying each subsystem as an independent problem has allowed to address the quantization of industrial-sized algorithms in reasonable times. The technique presented in $\left[\mathrm{WDJ}^{+} 06\right]$ proposes the partitioning of the system in its top-level blocks (FFT, channel equalizer, etc.). Then, the inputs and outputs of each of these entities are optimized to comply with the error constraint. Finally, a WLO process is carried out for each entity independently. This method is extended in [PRMS10b], where a multi-level hierarchical decomposition is applied. A budget of noise is distributed among the partitions, and the optimization is refined in each level of the partitioning. These methods allow the quantization of large systems but can miss optimization opportunities since treating each partitioned subsystem independently misses the global scope of the complete system.

\subsubsection{Quantization noise metrics}

The cost function to use for the WLO depends on the objective that is to be achieved. As stated in [PRMS10b], being $w$ a vector where each position corresponds to only one operation in the fixed-point implementation, $C(w)$ the closed form expression for total cost of the fixed-point implementation and $\lambda(w)$ its quantifying metric evaluation function, we can formalize the optimization process with the inequation

$$
\min (C(w)) \text { subject to } \lambda(w) \leq \lambda_{\max }
$$

where $\lambda_{\max }$ is the upper bound for the permitted quantization noise. It is possible to apply the inequation above with a variety of quantization noise metrics and cost functions considering different objectives for minimization, depending on the requirements of the design. In the following we overview different quantization noise metrics and optimization objectives.

Signal-to-Quantization Noise Ratio (SQNR) is one of the most widely used metrics to measure the influence of noise at the output of a quantized system. It relates the maximum signal strength and the contribution of the noise through the equation

$$
S Q N R=\frac{E[x]}{E\left[x_{N}\right]},
$$

where $E[x]$ is the signal power of the unquantized signal $x$ and $E\left[x_{N}\right]$ the signal power of the quantization noise. SQNR is an effective measurement for the quality of the solutions since the maximum allowed noise depends on the strength of the signal instead of being an absolute value. Some of the works that apply this metric are [HE06, SK95, $\mathrm{LGC}^{+} 06$, CCL03b, Con03, KS01, HEKC01, $\mathrm{CCC}^{+}$06, RMHS06, MRS08]. 
Many other works use simpler metrics in which simply the noise power which is provided by the noise variance $\sigma_{N}^{2}$ is measured and used as design constraint [LCCNT08, CB94, $\mathrm{CLL}^{+}$09, CC10b, CC10a, BCC05, BPCC09] or, in a few other cases, the mean square error (MSE) is used [CSPL01, CSL02, SB04c]. Since the main objective of these works is to present and demonstrate the validity of the complete approaches, the use of simplified noise metrics is acceptable as they could be replaced with more complex ones without invalidating the methodologies.

Finally, although its usage is not very extended, there are a few works that measure the Maximum Mismatch (MM) between the ideal values and the quantized signals in the system. This metric is applied in [SPR10, CVKF03, PRZ10, SR10, SR12, SR13]. Using the MM to evaluate the quantization noise is a robust but very conservative approach, since it is based on always considering the worst-case scenario.

\subsubsection{Optimization objectives}

One of the most common objectives of WLO is the minimization of the amount of hardware required to implement the target systems. This objective can be imposed by different motivations, from achieving small-sized chips and devices to the necessity of fitting a large design in an FPGA with limited resources. [HE06] demonstrates that the use of cost as minimization function outperforms error-oriented approaches. In order to reduce the hardware cost many works use the summation of word-lengths as guiding metric [WBGM97, $\mathrm{CRS}^{+}$99, CSPL01, CSL02, CFCNT04]. Although this approach does not guarantee the minimization of the hardware cost of the circuit, since it also depends on other factors ${ }^{1}$ that should be taken into account, the cost function is simple enough to justify its use in many cases.

The work presented in [CB94] constitutes an important step forward in this area. It introduces three different cost functions where area, power consumption and clock frequency (delay) are considered separately as minimization objectives. Results indicate that when delay is minimized the resulting word-lengths are almost uniform, which is not the case when area and power minimization are addressed. The authors also observe that area and power-oriented searches tend to be similar, demonstrating the direct effect of area on the power dissipation phenomenon.

Attempts to minimize the actual area used by the fixed-point designs have been carried out in [WP98, CCL03b, CB94, KS01, HE06, HEKC01, CSL02, CCC ${ }^{+}$06, CSPL01, SK95, SB04c, RMHS06], among others. Experimental results show that optimized MWL achieves important area reductions with respect to UWL, with reported improvements that go up to $13 \%$ in $\left[\mathrm{CCC}^{+} 06\right]$, near $30 \%$ in [HE06] and [RMHS06] or that range from $27 \%$ to $40 \%$ in [WP98]. Nevertheless, these values cannot be directly compared, because apart from the different WLO approaches, also the applications change. This can be a very determining factor, since each application offers different optimization

${ }^{1}$ For example, multipliers are larger and more expensive than adders. 
opportunities.

While optimizing the area usage, few works take into account the fact that the available resources may be of heterogeneous nature. But in the recent years, new FPGA designs have started including many specialized blocks, from DSP accelerators to fully functional microprocessors [Inc14]. The works in [Caf08, CC10a] take this in consideration and introduce the +-norm, which considers the different types of available resources and the capacity of the FPGA to contain the circuit while optimizing and allocating fixed-point designs. This leads to improvements up to $21 \%$ respect to other area-wise approaches.

Recently, power consumption has become a main concern in hardware design, and fixed-point system developers have not remained oblivious to this issue. Some works have studied the impact of quantization on power consumption [Con03, HESJ04, JCC08, CGC05]. Other authors have directly used this metric as minimization objective [TNR00, FCR02, GCC06, MSBZ07, CCC09], achieving power reductions up to 25-30\% with respect to UWL implementations. Interestingly, while some authors [CCL03b, Con03] indicate power improvements due to area optimization, others [GCC06] suggest that optimal area implementations can have a negative impact on the power consumption figures, even resulting in worse performance than UWL implementations.

Finally, few are the works [GML04, $\mathrm{LGC}^{+}$06, CCL03b, Con03, SR13] that measure the impact of quantization on latency and delay, and to the best of our knowledge only [CB94, CC10a] have used this metric as minimization objective.

\subsubsection{Mixed approaches}

Due to the complexity of finding optimized solutions, the majority of works previously reviewed in this section consider the quantization as an independent process, typically performed at an early stage in the HLS design flow. The most complex formulations attempt to optimize several metrics at once, moving through a Pareto-optimal front and selecting the most adequate solution according to the system constraints. But even in those cases, treating the quantization isolated from the rest of the HLS process may lead to missing optimization opportunities. Subsequent design stages like scheduling, allocation or binding, have a severe impact on the system final implementation. Thus, the function applied during quantization can only be a rough estimation of the actual cost.

Among the first works that attempted to palliate this problem by combining quantization with other stages of HLS, [WP98] performs WLO over a resource sharing architecture. The quantization is carried out independently from other design stages and area cost is used as optimization objective. The applied cost function is based on output latency and an innovative although too simple variable latency model.

The execution of a two-stage quantization interleaved with Architectural Synthesis (AS), is proposed in [KS01]. A first quantization, with a relaxed noise constraint, is 
performed before AS. Then, during AS, scheduling, resource allocation and binding are carried out to create a data path for the algorithm. Then, once the final structure is defined, an additional quantization is performed to comply with the noise requirements. To cope with the increased complexity of combining several HLS stages, the authors assume 1-cycle latency functional units and perform a single optimization iteration, among other simplifications that, while not invalidating the approach, may limit its applicability.

An improved methodology based on [WP98, KS01] is developed in [HMS07]. Instead of only iterating once, the proposed method loops over AS and WLO several times. In the WLO stage, operations are grouped according to word-length and mobility-related criteria and quantization is applied to each group individually. Modifying word-lengths generates a new data path, which is analysed again, and the grouping and optimization is repeated until the solution cannot be further improved. Also, the cost estimation for each operator is a function of the area, latency and power consumption, all of them dependant of the word-length.

A different approach is followed in $\left[\mathrm{CCC}^{+} 06\right]$, where quantization and $\mathrm{AS}$ are combined through MILP. Again, similarly to [KS01], some simplifications are required to allow the execution times to remain affordable. In this case, 1-cycle latency functional units are assumed, and elements like multiplexers and registers are not considered in the process. The lack of real-life applicability is compensated by the fact that the the conclusions and heuristics derived from this work are applied in later works [CC10a, CC10b] that extend and improve the methodology. In these approaches, simulated annealing is used to integrate SA and quantization for FPGA-oriented circuits in a single HLS stage, achieving area improvements of around $21 \%$ compared to traditional methods.

Other works, instead of focusing on the combination of quantization with high-level transformations, attempt to perform fine-grain optimizations on the functional blocks that integrate the destination platform. It is the case of [BCC05, BPCC09], where quantization is combined with the binding of FPGA resources and arithmetic operations. The order in which operations are assigned to resources is driven by the precision requirement for each of them: the operations with larger word-lengths are bound first. Through this methodology the use of the available resources in an FPGA is improved.

\subsection{Computational scalability}

As the size of the systems to implement in fixed-point arithmetic increases, analytical approaches as the ones presented in the previous sections are replacing simulation-based methods for small and medium sized systems. But in order to model accurately more complex operations, the analytical models are gradually becoming more convoluted. Added to the increment in the number of operations and the uncertainties modelled during evaluation, this leads to large and complex mathematical equation systems that even modern computers have difficulties to solve in reasonable times. Consequently, 
analytical approaches that attempt to be used in real world industrial applications must not only be as general as possible but also scalable. But only recently have researchers started to address this issue, and the body of work on this behalf is limited.

The authors in [PRMS10b] approach system scalability through a divide-and-conquer technique. The system to study is hierarchically divided into sub-blocks and each of them is studied through the Single Noise Source Model that calculates the probability density function (PDF) and power spectral density (PSD) for each block. These computations can be quite complex, as an analysis of auto-correlation and cross-correlation is required to obtain the characteristics of each system. This technique is later extended in $\left[\mathrm{NTA}^{+} 13\right]$ so that systems with multiple outputs can be decomposed into individual noise models.

A different way to achieve a scalable model is by limiting the size of the equations used to represent it. This approach is followed in [BC12, BC13], where Handelman representations are used to model the round-off noise. Inputs and intermediate values through the system are represented as polynomials consisting of a sum of weighted terms where each term is a product of a variable raised to some integer power. While propagating the values through the system, every time a polynomial exceeds a given number of terms, the least significant terms (i.e. those with the smallest potential contribution which is calculated computing the bounds of each term using IA) are replaced by a new single term centred over the desired range. This method achieves low execution times even for large systems, since the proliferation of terms is strictly controlled, but a large number of replacements will unavoidably translate into accuracy degradation of the final results.

There are also opportunities for scalable analysis if systems are analysed from a higher abstraction level. The authors in [DYSD14] apply the polyhedral model, a framework for automatic optimization and parallelization of algorithms, to generate Systems of Recurrence Equations from which it is possible to extract convolutions from the equational view of the program. These are used to generate a graph of the system to which the noise sources are then added, and the noise power is calculated. This approach does not treat non-LTI operators, but the authors discuss that different approaches where this type of elements are approximated by linearisation could be integrated in this solution. Also, this approach is not applicable if the system to analyse is non-polyhedral.

\subsection{Fixed-point tools and frameworks}

In order to ease the development of increasingly larger and more complex electronic systems, the generalized trend of EDA design is to gradually relieve the designer from low-level implementation issues and decisions and rely on automated procedures to perform them, while leaving only high-level issues in the hands of the designer. In this context, quantization and WLO are not strange to this trend. Given the tight relation and high impact this design stage can have in the quality of the final implementation, and the fact that later design stages can even invalidate the optimization results, thus requiring a new analysis and reassignment of word-lengths, the development of automa- 
ted quantization and WLO tools and frameworks has been a central topic of interest in the research community for several years. This section covers a number of fixed-point frameworks developed in academic environments.

One of the first proposed WLO environments is the FRIDGE framework [WBGM97]. Using ANSI-C algorithms as input, an extension called fixed-C is introduced to represent fixed-point data types, where bit widths for both integer and fractional parts are explicitly described. Compiler support for this extension is provided through the Fixed-C Compiler (FCC) [JW98]. The framework allows the designer to provide a non-exhaustive specification of the fixed-point format in the system and then, automatically, performs a suitable assignment of word-lengths to the remaining signals. This is performed by means of the HYBRIS simulator, which is capable of simulating together fixed- and floating-point arithmetic formats. The FRIDGE environment can generate optimized output descriptions in ANSI-C for DSP processors and VHDL code. The tool was included in the CoCentric hardware/software co-design suite by Synopsys. An extension that included Interval Arithmetic for the estimation of range an precision was later proposed in [CKLM02].

To overcome the drawback of long simulation times as required in the first proposals of the previous work, a quasi-analytical design environment was presented in [CRS $\left.{ }^{+} 99\right]$. In this work, dynamic ranges and quantization errors are propagated through the system along with the computations of a high precision simulation. Using overloaded operators, it is possible to perform fixed-point refinement while the simulation runs. This mixed evaluation guarantees that both floating-point and fixed-point values will take the same control decisions through the simulations. The methodology is simple and fast, but it does not attempt to obtain optimized solutions.

The $A B A C O$ set of tools [CLNT99] allows the analysis and verification of functions implemented in fixed-point arithmetic. The tools, developed in $\mathrm{C}++$, take advantage of the GMP (GNU Multi-Precision arithmetic library) to simultaneously perform the simulation of operations with multiple precisions. The environment, based on discrete or variable-precision interval-based data types, was extended to grant support to DSP systems and algorithms with feedback loops in [Ló04]. Nevertheless, this framework only covers the evaluation of quantized systems, and no methods to optimize the word-length assignments are included.

Another framework is presented in [CCL00] under the name of Synoptix. It uses data flow descriptions of Matlab's Simulink as inputs to the tool and generates optimized fixed-point descriptions that are fed to a HDL synthesis tool that, using custom fixedpoint operator libraries, generates the final hardware implementations. This framework allows the selection of different noise metrics, such as $L_{1}$ norm, and applies a $m i n+1$ greedy algorithm in combination with MILP to carry out the word-length optimization.

The Précis tool [CH02], unlike other frameworks in this section, is not meant as an automated optimization framework, but as an aid to the designer to evaluate the precision requirements of the algorithm under development and to decide the word- 
lengths to assign to each variable in the system. The input specification for the tool is a Matlab description. It provides a constraint propagation engine, support for simulation, the capability of determining ranges and a very relaxed precision estimation. This tools are meant to provide hints about the expected precision requirements of the algorithm under development, but the ultimate decisions about the word-length assignments lie in the hands of the designer.

The BitSize optimization tool is presented in [GML04]. It applies Automatic differentiation (AD) to analyse the bit width requirements of the algorithm, and allows the optimization of both fixed- and floating-point systems. AD is an applied mathematical method that has the ability to calculate the differentials of a function as a side effect of the execution of the function itself. By means of this method, the sensitivity of each node of the system can be calculated. Two different noise models are applied, depending of whether the target is a floating- or a fixed-point system. The BitSize tool proposes a two-pass execution for the optimization procedure. First, a forward analysis to perform the $\mathrm{AD}$ of the system nodes. Second, a backward pass that uses the sensitivity information previously calculated to optimize the word-lengths of the system variables. The framework where the described methodology is applied offers the possibility of using $\mathrm{C} / \mathrm{C}++$ or Xilinx System Generator (XSG) design descriptions as inputs to the framework, and produces annotated data flow graphs that can be synthesized with XSG or verified in Matlab.

FFC, a set of tools fully integrated with Simulink and Matlab, is presented in [SB04a]. It relies on the help of the designer to operate, and different Simulink blocks are defined to this end. A Range Detector is used to collect information about dynamic ranges during a running simulation. It has to be manually placed in the design and the tool removes it automatically after the dynamic range evaluation is performed in order to continue with the optimization process. Another critical Simulink block that has to be placed by the developer is the Spec Marker, which is used to enter user specifications of the system performance in the critical blocks of the data path. The integration with Simulink enables the FFC tools to evaluate the hardware cost accurately. MSE is used as noise metric, but since occasionally more precise metrics are required, the use of custom Matlab functions is allowed. In order to reduce the solution space to explore and thus improve optimization times, signal grouping can be used.

The Fixify toolset [BR04, BR05] presents an environment with a large variety of design options. It is capable of recognizing and parsing input system descriptions in SystemC, VHDL and Synopsys' COSSAP formats, it offers several options to specify the error constraint (SQNR, BER, SER) and the possible implementation costs (area, power, throughput). It also lets the designer to choose the optimization strategy: Full search, restricted full search, greedy search (in a backward fashion, starting with the optimization of the outputs and moving back until the inputs are reached) and a branch-and-bound algorithm. The main drawback of this quantization environment is that it relies on simulation techniques to obtain the quantization noise values of the different word-length 
configurations. This can greatly penalize the execution time of the exploration required for the solution space.

A multi-staged framework called LengthFinder is introduced in $\left[\mathrm{OCC}^{+} 07\right]$. In order to deal with the trade-off between accuracy and execution times, the optimization is divided in low- and high-effort passes. First, a range analysis based on IA and AA is executed. Then, several techniques (coarse-grain analysis, generation of cost tables and heuristic partitioning) are applied to reduce the search space. Then, the low-effort optimization pass based on greedy searches (both $\min +1$ and $\max -1$-see Section 2.2.1- are available) is carried out. Optionally, a high-effort genetic search can then be done starting from the previously obtained results in order to further refine the final solution. The fact that the tool uses AA for range analysis and the lack of a clear description of the approach to evaluate precisions cast some doubts about the extent of applicability of this framework.

One of the most modern proposed frameworks is the ID.Fix tool [MSNS12]. It is built using the GECOS (GEneric COmpiler Suite) compiler infrastructure and uses annotated $\mathrm{C} / \mathrm{C}++$ source code as input. In this environment, pragmas are used to indicate suggested dynamic ranges for the input values. It applies the methodology described in [MRS08] to analyse the quantization error. The environment offers a modular structure to enable the evaluation of different optimization heuristics. The optimized fixed-point formats are used to generate output files in $\mathrm{C}$ that make use of the ac_fixed data type which are used by Catapult $\mathrm{C}$ to generate hardware implementations of the algorithms.

In this section we do not review commercial frameworks, like the discontinued AccelDSP [Inc15] (now integrated in the VIVADO suite), since in this Ph.D. Thesis we only focus on academical tools.

\subsection{Conclusions}

Word-length optimization is a complex field of research that has attracted the interest of many researchers through the years. Given the variety of applications that benefit from the use of fixed-point arithmetic, multiple optimization objectives, constraints and metrics have been targeted, and a multitude of methodologies have been developed.

In this chapter we have reviewed classical and modern techniques for signal and round-off noise modelling. We have seen how analytical techniques, even though faster than simulation-based ones, are limited in the types of operations they can model, and often suffer from scalability issues. Also, we have seen how only the work in [Wu12] addresses the dynamic range estimation of systems with control-flow structures in them. Given the number of real life applications that include this type of structures, not being able to model them accurately is an important shortcoming of the overviewed models.

Also, we have seen different optimization methods that include search algorithms that go from exhaustive to greedy ones, and we have observed how these can also vary greatly, from heuristic search algorithms to mathematical modellings of the optimization problem. Also we have described mixed approaches, where fixed-point optimization is 
combined with other objectives in an attempt to solve them all at once.

In summary, we have been able to certify that the problem of word-length optimization has been approached from very different perspectives. And after going over a number of academia-oriented optimization frameworks we have established that none of them has the potential to give support for all of those approaches.

In this Ph.D. Thesis we address several of the shortcomings identified on this chapter. We focus our work in developing inclusive analytical methods that allow us to create accurate models of the impact of round-off noise in the systems under study, even if these systems include control-flow structures or discontinuities and, to the extent possible, regardless of the problem size. We also contribute with new optimization techniques capable of solving the simulation-based optimization problem several orders of magnitude faster than other greedy methods. Finally, we introduce HOPLITE, an open word-length optimization framework designed to be modular and extensible, and to allow developers to implement and validate their approaches with minimal effort. 


\section{Chapter 3}

\section{Quantization of systems with control flow structures}

The way to deal with an impossible task was
to chop it down into a number of merely very
difficult tasks, and break each one of them
into a group of horribly hard tasks, and each
of them into tricky jobs, and each of them...
Terry Pratchett,
Truckers

The numerical accuracy at the output of a system implemented using fixed-point arithmetic is commonly used as a metric to evaluate the performance of the circuit. As we saw in the previous chapter, automatic analysis using models has gradually replaced simulation-based methodologies when studying systems with certain characteristics. But despite the quick evolution and improvement of the analytical models over the past years, their scope is still constrained due to several factors like accuracy in presence of strongly non-linear operators, scalability and control-flow structures, among others.

In this chapter we introduce a novel approach for modelling the dynamic range and the round-off noise of digital systems with control-flow structures. To do that, we develop a methodology based on the Multi-Element generalized Polynomial Chaos (MEgPC) model, which has proven to provide accurate results for dynamic range analysis in strongly non-linear systems [WK05]. Inspired by its principles, we have developed an automated procedure for analysing all possible execution paths independently and then combine the results to obtain the dynamic range of the complete system. Then, we extend that approach to encompass the estimation of round-off noise, thus providing a comprehensive approach for the analytical modelling of fixed-point systems in the presence of control-flow structures.

We also approach the problem of the combinatorial explosion of the size of polynomials as problems grow in complexity, which leads to scalability issues. To solve this we 
present a technique based on grouping noise sources in small clusters, then propagating them independently, and finally combining the partial results. This approach allows us to control the maximum number of variables in the system, effectively limiting the size of the polynomials to manageable numbers.

The rest of the chapter is organized as follows: Section 3.1 overviews the theoretical background of affine arithmetic, modified affine arithmetic, polynomial chaos and ME-gPC theory. In Section 3.2 we define control-flow structures and present our methodology for modelling the dynamic range of systems that contain them. This approach is extended to estimate round-off noise as well in Section 3.3. Finally, we address the issue of scalability in Section 3.4. There, we outline our technique for grouping noise sources and we propose a partitioning algorithm to minimize the loss of accuracy that the application of this approach may cause.

These methodologies are later illustrated in Chapter 4 through different case studies, showing also how to combine them into a single flow.

\subsection{Theoretical background}

The evaluation of dynamic range and RON using extensions of interval simulations has been progressing during the past years and different new methodologies have been proposed to improve the quality and accuracy of the solutions as well as to broaden the scope of the systems that can be addressed using them.

The first of such extensions is AA. It was proposed to estimate the SQNR and perform the quantization of LTI systems [FRPC03, Ló04]. But, as mentioned in Section 2.1, AA fails to capture correlations between signals after non-linear operations[Ló04]. To overcome this issue, MAA was proposed [LCNT07, Est11]. The complexity of this method lies in the fact that its affine forms are not normalized, and consequently the propagation of the affine terms becomes a complex task.

The use of normalized affine forms for modelling non-linear operators is introduced by PCE, where the signals are decomposed in terms of orthogonal polynomials, thus allowing easy propagation of the coefficients through the Data Flow Graph (DGF) [WZN06, ELSS12]. This approach has been applied to both dynamic range estimation in [WZN06], and to the analysis of the RON for small, sequential systems in [ELSS12] by taking advantage of the possibilities offered by the Wiener-Askey scheme. But PCE still fails to deal efficiently with discontinuities and it is not capable of modelling controlflow operations. ME-gPC, on the other hand, is able to produce accurate models for discontinuous systems, as we explain below. In this section we review the mathematical processes of AA, MAA, PCE and ME-gPC, which will serve as basis for the techniques we propose in the subsequent sections of the chapter. 


\section{CHAPTER 3. QUANTIZATION OF SYSTEMS WITH CONTROL FLOW STRUCTURES}

\subsubsection{Affine arithmetic}

An affine form is defined as a polynomial expansion of order one where the independent variables are uniformly distributed in the interval $[-1,1]$. Affine arithmetic is capable of capturing the correlation between intervals after affine operations (i.e. linear). A first order affine form is expressed as [LCCNT08]:

$$
\hat{a}=a_{0}+\sum_{i=1}^{n_{a}} a_{i} \cdot \epsilon_{i}
$$

The mean value is given by $a_{0}$, the terms $\epsilon_{i}$ are the independent sources of uncertainty and the coefficients $a_{i}$ are the amplitudes of these uncertainties. The uncertainty sources can represent the variations of the signal or the RON. The basic operations between two affine forms $\hat{a}$ y $\hat{b}$ considered by AA are summarized in Table 3.1 [SDF97]. The instructions supported by this methodology are either linear [Ló04] or smooth non-linear [Caf08], meaning that their behaviour can be approximated by linear models. The terms $n_{\max }$ refer to the maximum number of noise terms present in the affine forms.

Table 3.1: Coefficient propagation rules of Affine Arithmetic.

\begin{tabular}{|c|c|}
\hline Operation & Coefficient propagation rule \\
\hline \hline Addition & $\hat{a}+\hat{b}=\left(a_{0}+b_{0}\right)+\sum_{i=1}^{\max \left(n_{a}, n_{b}\right)}\left(a_{i}+b_{i}\right) \cdot \epsilon_{i}$ \\
\hline Subtraction & $\hat{a}-\hat{b}=\left(a_{0}-b_{0}\right)+\sum_{i=1}^{\max \left(n_{a}, n_{b}\right)}\left(a_{i}-b_{i}\right) \cdot \epsilon_{i}$ \\
\hline Constant multiplication & $c \cdot \hat{a}=c \cdot a_{0}+\sum_{i=1}^{n_{a}} c \cdot a_{i} \cdot \epsilon_{i}$ \\
\hline Multiplication & $\hat{a} \cdot \hat{b}=\left(a_{0} \cdot b_{0}\right)+\sum_{i=1}^{\max \left(n_{a}, n_{b}\right)}\left(a_{0} \cdot b_{i}+a_{i} \cdot b_{0}\right) \cdot \epsilon_{i}+$ \\
& $\left(\sum_{i=1}^{n_{a}}\left|a_{i}\right| \sum_{i=1}^{n_{b}}\left|b_{i}\right|\right) \epsilon_{n_{\max }+1}$ \\
\hline Rounding & $Q_{f}^{R}(a)=\left(a_{0}-2^{-f-1}\right)+\sum_{i=1}^{n_{a}} a_{i} \cdot \epsilon_{i}+2^{-f-1} \cdot \epsilon_{n_{\max }+1}$ \\
\hline Truncation & $Q_{f}^{T}(a)=a_{0}+\sum_{i=1}^{n_{a}} a_{i} \cdot \epsilon_{i}+2^{-f-1} \cdot \epsilon_{n_{\max }+1}$ \\
\hline
\end{tabular}

Linear operations (addition, subtraction and constant multiplication) are executed in a precise manner. However, after performing nonlinear operations the temporal correlations of the input signals are lost [WZN06]. The result of executing non-linear operations over uniform distributions is typically non-uniform so it is theoretically impossible to represent it as a linear combination of uniform distributions. In order to alleviate this shortage other methods arise. In particular MAA [SLMW03] introduces higher order polynomials to capture the correlations among the signals.

\subsubsection{Modified affine arithmetic}

In [SLMW03] and [SLMW04], MAA is used for bivariate polynomial evaluation and algebraic curve plotting in $2 \mathrm{D}$. However in these works the only operations treated are multiplications.

Given two affine forms 


$$
\begin{aligned}
& \hat{a}=a_{0}+a_{1} \cdot \epsilon_{a}, \\
& \hat{b}=b_{0}+b_{1} \cdot \epsilon_{b},
\end{aligned}
$$

$\epsilon_{a}$ and $\epsilon_{b}$ are the noise terms bounded in the interval $[-1,1], a_{0}$ and $b_{0}$ are the means of both variables and $a_{1}$ and $b_{1}$ represent the variations of the signals over the mean values. The simplest nonlinear operation is a multiplication of both affine forms:

$$
f(\hat{a}, \hat{b})=\hat{a} \cdot \hat{b}=a_{0} \cdot b_{0}+a_{0} \cdot b_{1} \cdot \epsilon_{b}+a_{1} \cdot b_{0} \cdot \epsilon_{a}+a_{1} \cdot b_{1} \cdot \epsilon_{a} \cdot \epsilon_{b} .
$$

Generalizing for any order, the centred form of the output polynomial is given by

$$
f(\hat{a}, \hat{b})=\sum_{i=0}^{n-1} \sum_{j=0}^{m-1} a_{i} \cdot b_{j} \cdot \epsilon_{a}^{i} \cdot \epsilon_{b}^{j} .
$$

It can be seen that this solution is an extension of AA in which all the high order terms are taken into account. In [SLMW04], this technique is just applied in the case of multiplications and other non-linear operations are obviated. Nevertheless, since the monomials of MAA are not orthonormal, the incorporation of the PCE techniques that take into account higher order terms when considering different types of operations is also required. Without them, the propagation of the gains throughout the system under analysis would not be accurately performed.

\subsubsection{Polynomial chaos expansion}

Given a set of independent random variables of dimension $N, \Phi=\left\{\phi_{1}, \phi_{2}, \cdots, \phi_{N}\right\}$ and another random variable $Y$, square integrable that is a function of $\Phi, Y=f(\Phi)$, then $Y$ can be expressed as a weighted sum of polynomials that are orthogonal with respect to the joint probability density function of the input random vector $\Phi$ as

$$
Y=\sum_{i}^{\infty} \alpha_{i} \cdot \psi_{i},
$$

where each $\alpha_{i}$ is a constant coefficient and each $\psi_{i}$ is the $i$-th polynomial from an orthogonal basis [WZN06]. In practice, the number of terms of the expansion should be truncated to a finite number $m$. This number depends on the dimension of the expansion $n$ (number of independent variables in vector $\Phi$ ) and the maximum order of the polynomials used, $p$. The terms $\alpha_{i}$ are the spectral coefficients of the expansion and the terms $\psi_{i}$ are orthogonal polynomials which satisfy the condition

$$
<\psi_{i} \cdot \psi_{j}>=\left\{\begin{array}{cl}
<\psi_{i}^{2}>, & \text { if } i=j \\
0, & \text { if } i \neq j
\end{array} .\right.
$$

All orthogonal polynomials basis can be built by repeatedly applying the Rodriguez formula [XL02]: 
Table 3.2: Types of RVs and their associated sets of orthonormal polynomials.

\begin{tabular}{|c|c|c|}
\hline RV & Orthonormal Polynomials & Support \\
\hline \hline Gaussian & Hermite & $(-\infty, \infty)$ \\
\hline Uniform & Legendre & {$[a, b]$} \\
\hline Gamma & Laguerre & {$[0, \infty)$} \\
\hline Beta & Jacobi & {$[a, b]$} \\
\hline
\end{tabular}

$$
\psi_{i}=\frac{1}{\beta_{n} \omega(\phi)} \frac{d^{p}}{d \phi^{p}}\left[\omega(\phi) S(\phi)^{n}\right] .
$$

Depending on the density of the measure of $\phi$, often called weighting function $\omega(\phi)$, several families of orthogonal polynomials can be generated. $\beta_{n}$ is a standardization factor and $S(\phi)$ is a polynomial of first or second degree at most. In practice, the polynomials to generate the basis selection depends on the probability density functions of the RVs that are present in the system. In Table 3.2 we show some families of orthogonal polynomials [XK03].

Hermite polynomial chaos employs gaussian random variables and Hermite orthogonal polynomials. The generating function for dimension one and order $p$ is

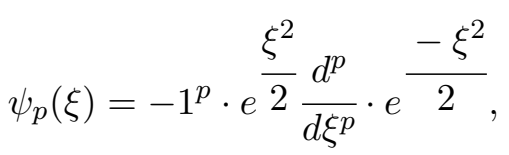

where $\xi$ is a zero-mean gaussian orthonormal random variable. With Eq. 3.9 a set of polynomials $\Psi=\left[\psi_{1}(\xi), \psi_{2}(\xi), \cdots, \psi_{m}(\xi)\right]$ of one variable up to order $p$ can be built by just repeatedly substituting the values of $p$ in Eq. 3.9. In this particular case the number of polynomials $m$ matches with the order of the expansion $p$ because just one independent variable $\xi$ is employed, but this is not the general case. To build a polynomial basis that depends on several random independent variables, Eq. 3.9 can be used to obtain the correspondent one dimension set of univariate polynomials. The Multivariate Hermite polynomials are obtained making the tensorial product of these sets of univariate polynomials.

Being $\Xi=\left[\xi_{1}, \xi_{2}, \cdots, \xi_{n}\right]$ the input Gaussian stochastic space, the coefficients of the expansion $\alpha_{i}$ in Eq. 3.6 can be determined using a Galerkin projection operation [WZN06, $\mathrm{DNP}^{+}$04]

$$
\alpha_{i}=\frac{<Y \psi_{i}(\Xi)>}{<\psi_{i}^{2}(\Xi)>}
$$

which can be resolved using Monte-Carlo techniques provided that enough samples of the stochastic set $\Xi$ are available and an input-output relationship $Y^{M}=f(\Xi)$ can be established.

In [WZN06] it is proposed to use this scheme to estimate the dynamic range of 
non-linear digital data-paths. Since the input stochastic space may not be gaussian it is proposed to transform it using the inverse error function $\Xi=\phi^{-1}(Z)$ into an standardized random vector of gaussian RVs $\Xi$, where $Z$ is a set of RVs of a given distribution. This procedure is also addressed in [BSB07] and is valid under certain statistical conditions of the RVs contained in $Z$, [WZN06] and [XK02].

Once the input signals are defined, the next step is to propagate the coefficients through the data flow graph. This procedure is done exploiting the orthogonality properties of the polynomials. The propagation of the polynomial chaos coefficients through operations is well detailed in [WZN06] and $\left[\mathrm{DNP}^{+} 04\right]$, and the basic operations are summarized below. Consider two input RVs $\hat{x}$ and $\hat{y}$ expanded in a PCE:

$$
\begin{aligned}
& \hat{x}=\sum_{i=1}^{m} x_{i} \cdot \psi_{i}, \\
& \hat{y}=\sum_{i=1}^{m} y_{i} \cdot \psi_{i} .
\end{aligned}
$$

The computation of the linear operations is straightforward. In the case of multiplying $\hat{x}$ by constant $c$, the coefficients of the new PCE are

$$
\hat{z}=\sum_{i=1}^{m} c \cdot x_{i} \cdot \psi_{i} \Rightarrow z_{i}=c \cdot x_{i} .
$$

The addition and subtraction operations are performed just by adding or subtracting the correspondent coefficients of the input PCE:

$$
\hat{z}=\hat{x} \pm \hat{y}=\sum_{i=1}^{m}\left(x_{i} \pm y_{i}\right) \cdot \psi_{i} \Rightarrow z_{i}=x_{i} \pm y_{i} .
$$

Propagation through non-linear operations such as multiplication and division is not so direct. Considering that $\hat{z}=\hat{x} \cdot \hat{y}$ and by substituting each variable by its correspondent PCE, it is obtained that

$$
\sum_{k=1}^{m} z_{k} \psi_{k}=\sum_{i=1}^{m} x_{i} \psi_{i} \sum_{j=1}^{m} y_{j} \psi_{j}
$$

where it is considered that all the expansions are of the same length $m$. Doing a Galerkin projection $\left[\mathrm{DNP}^{+} 04\right]$ on $(3.15)$, the coefficients $z_{k}$ are calculated as:

$$
z_{k}=\sum_{i=1}^{m} \sum_{j=1}^{m} \frac{<\psi_{i} \psi_{j} \psi_{k}>}{<\psi_{k}^{2}>} x_{i} y_{i}=\sum_{i=1}^{m} \sum_{j=1}^{m} C(i, j, k) x_{i} y_{i},
$$

which constitutes a linear system of $m$ equations, expressed in matrix form as

$$
Z=A \cdot X
$$




$$
A=C \cdot Y \text {. }
$$

$A$ is an $m \times m$ matrix and $X, Y$ and $Z$ are column vectors correspondent to the $\hat{x}, \hat{y}$ and $\hat{z}$ coefficients, respectively. Taking Eq. 3.17 in the reverse direction the computation of the division can be executed easily ensuring that the inverse of matrix $A$ is calculated. Tensor $C(i, j, k)$ is the same for a given dimension and order, so it only has to be calculated once, for instance in a pre-processing stage, and afterwards reused when needed, thus notably reducing the required computation time. Appendix A shows techniques for accelerating the computation of the $\mathrm{C}$ matrix, speeding the overall process even further.

An important question is how to determine the required order of the PCE polynomials before carrying out the computations (which is required to generate the polynomial base and computing the C matrix). This can be easily done by means of traversing the DFG of the system under consideration. The order required by each linear (additions, subtractions, constant multiplications) is the same as the maximum order of its predecessors, while the order required by non-linear operations (non-linear multiplications and divisions) is equals to the sum of the orders of its predecessors.

Since PCE polynomials are orthonormal, it is possible to reuse the expressions obtained when combining PCE and MAA to calculate the noise contributions at the output of a system and optimize fixed-point word-lengths fast and efficiently [Est11]. Here it is already evident that a methodology for reducing the number of PCE terms for this type of analysis is required in order to address larger designs.

The interested reader may find detailed examples of the propagation of affine forms using combined PCE + MAA in [WZN06, Est11].

\subsubsection{Multi-element generalized polynomial chaos}

In many cases, PCE requires excessively large basis to accurately represent the set of values. This happens particularly in the presence of discontinuities, or when many non-linear operations appear following each other. To overcome this, ME-gPC is formulated in [WK05]. This technique partitions the input domain in smaller sub-domains, decomposing complex functions in a set of simpler ones. This effectively allows for the use of lower PCE orders while modelling each sub-domain while providing very accurate results.

Being $B=[-1,1]^{n}$ the domain in which $\Xi=\left[\xi_{1}, \xi_{2}, \ldots, \xi_{n}\right]$ is defined, the ME-gPC method proposes its decomposition in $E$ non-overlapping elements. The domain of each element will be contained within $B_{k}=\left[a_{1}^{k}, b_{1}^{k}\right) \times\left[a_{2}^{k}, b_{2}^{k}\right) \times \ldots \times\left[a_{n}^{k}, b_{n}^{k}\right]$ where $a_{i}$ and $b_{i}$ are respectively the upper and lower bounds of the $i$-th local random variable.

Resulting from this decomposition, a local random vector for each element is defined as $\zeta^{k}=\left[\zeta_{1}^{k}, \zeta_{2}^{k}, \ldots, \zeta_{n}^{k}\right]$, subject to a PDF $f_{\zeta^{k}}=\frac{1}{2 J_{k}}$ where

$$
J_{k}=\prod_{i=1}^{n} \frac{b_{i}^{k}-a_{i}^{k}}{2}
$$


In order to take advantage of the orthogonality and properties of Legendre Chaos, each $\zeta^{k}$ is replaced by a new random vector $\xi^{k}=\left[\xi_{1}^{k}, \xi_{2}^{k}, \ldots, \xi_{n}^{k},\right]$, equivalent to $\zeta^{k}$ but in the domain $[-1,1]^{n}$ instead of $B_{k}$. Both random vectors are related according to the following relationship:

$$
g_{k}\left(\zeta^{k}\right): \zeta_{i}^{k}=\frac{b_{i}^{k}-a_{i}^{k}}{2} \xi_{i}^{k}+\frac{b_{i}^{k}+a_{i}^{k}}{2} .
$$

Once a dimension has been partitioned, new PCE expansions for each sub-domain are generated. Each of these expansions is in the form

$$
\tilde{u}(\tilde{\xi})=\tilde{u}(g(\hat{\xi}))=\sum_{i=1}^{m} \tilde{u}_{i} \Phi_{i}(\tilde{\xi})
$$

with $\tilde{\xi} \in[-1,1]^{d}$. To calculate the coefficients $\tilde{u}_{i}$ of each new expansion a linear system is solved. This system is generated choosing $m+1$ uniform grid points $\tilde{\xi}_{i}$ in $[-1,1]^{d}$ and has the form

$$
\left[\begin{array}{cccc}
\Phi_{0,0} & \Phi_{1,0} & \ldots & \Phi_{m, 0} \\
\Phi_{0,1} & \Phi_{1,1} & \ldots & \Phi_{m, 1} \\
\vdots & \vdots & \vdots & \vdots \\
\Phi_{0, m} & \Phi_{1, m} & \ldots & \Phi_{m, m}
\end{array}\right]\left[\begin{array}{c}
\tilde{u}_{0} \\
\tilde{u}_{1} \\
\ldots \\
\tilde{u}_{m}
\end{array}\right]=\left[\begin{array}{c}
\sum_{i=1}^{m} \hat{u}_{i} \Phi_{i}\left(g^{-1}\left(\tilde{\xi}_{0}\right)\right) \\
\sum_{i=1}^{m} \hat{u}_{i} \Phi_{i}\left(g^{-1}\left(\tilde{\xi}_{1}\right)\right) \\
\ldots \\
\sum_{i=1}^{m} \hat{u}_{i} \Phi_{i}\left(g^{-1}\left(\tilde{\xi}_{m}\right)\right)
\end{array}\right]
$$

where $\Phi_{i, j}=\Phi_{i}\left(\tilde{\xi}_{j}\right)$. With the expansions $\tilde{u}(\tilde{\xi})$ obtained with this method, PCE can be locally applied to the different elements. Once the expansions have been computed, the statistical global moments can be reconstructed from the different approximations applying Bayes' theorem and the law of total probability

$$
\mu_{m}(u(\xi))=\int_{B} u^{m}(\xi)\left(\frac{1}{2}\right)^{d} d \xi \approx \sum_{k=1}^{N} \operatorname{Pr}\left(z_{k}=1\right) \int_{[-1,1]^{d}} \hat{u}_{k}^{m}\left(\xi^{k}\right)\left(\frac{1}{2}\right)^{d} d \xi^{k}
$$

While the partitioning described here can be statically performed at a pre-processing stage, the authors in [WK05] propose a dynamic adaptive criterion to do it efficiently. Detailed explanations on the algorithm and the parameters in it can be found in [WK05, WK06].

So far MEgPC has only been used to estimate the dynamic range in systems without control-flow structures, and it has been only applied to numerical procedures. In the next sections we present a novel general methodology combining ME-gPC and MAA for estimating the dynamic range and the RON in digital systems with control-flow structures, extending the initial analysis carried out for linear systems in [LCCNT08] and for non-linear operations in [CCLF10, Est11] to systems with control structures in 
the Data Flow Graph. This broadens largely the applicability of the probabilistic interval analysis in word-length optimization, as it allows for an entire new class of systems to be targeted for dynamic range and RON modelling and optimization.

\subsection{Dynamic range estimation in systems with control- flow structures}

The use of PCE allows to generate very accurate models of complex systems, as previous works have demonstrated [WZN06, Est11]. But the methodology does not contemplate systems with control-flow operators. These constructs are extremely common in real-life applications, and the range of systems that can benefit from any approach that does not consider them is severely limited. In addition to the analysis of systems with discontinuities, the ideas introduced by the ME-gPC methodology can help us to represent this type of structures accurately.

In this section we present a novel scheme for estimating the dynamic ranges and round-off noises of systems that contain control-flow structures. Our methodology automatically partitions the input domain, matching sub-domains with different execution paths in the system description. By doing this, we can then reconstruct the system statistics in the same way ME-gPC does. As such, this work largely extends the applicability of the ME-gPC methodology.

A similar technique only applied to dynamic range modelling, has been presented in [Wu12]. In this approach, the Hermite PCE is extended to propagate the affine forms through control-flow structures by means of adding conditional probabilities to each branch of the control-flow structures. The result of this model is a single expression that adds the propagations through all the branches subject to their probability. Being the combinatorial explosion of terms one of the main drawbacks of polynomial-based techniques, as discussed in Section 3.4, this approach does not fit particularly well for the analysis of this type of structures in the general case, since the combinatorial explosion is very largely increased when using the same polynomial for all the possible execution paths. Instead, we propose a methodology based on ME-gPC using Legendre polynomials, where we partition the input domain so that each partition only maps one execution path. This results into a larger number of simpler equations that we later combine, so the overall computation is simpler, particularly in complex structures. In addition, while in [Wu12] loop structures and choice operators are treated differently, our methodology approaches them uniformly, thus simplifying the overall scheme.

The proposed process is summarized in Algorithm 1. Starting from the initial system graph, we look for the first control-flow structure and we generate derivative subsystems as detailed in Section 3.2.1. Then we propagate the input PCE coefficients through the initial system graph until we reach the left- and right-hand sides of the equation that controls the condition of the control-flow structure and we partition the input domain 


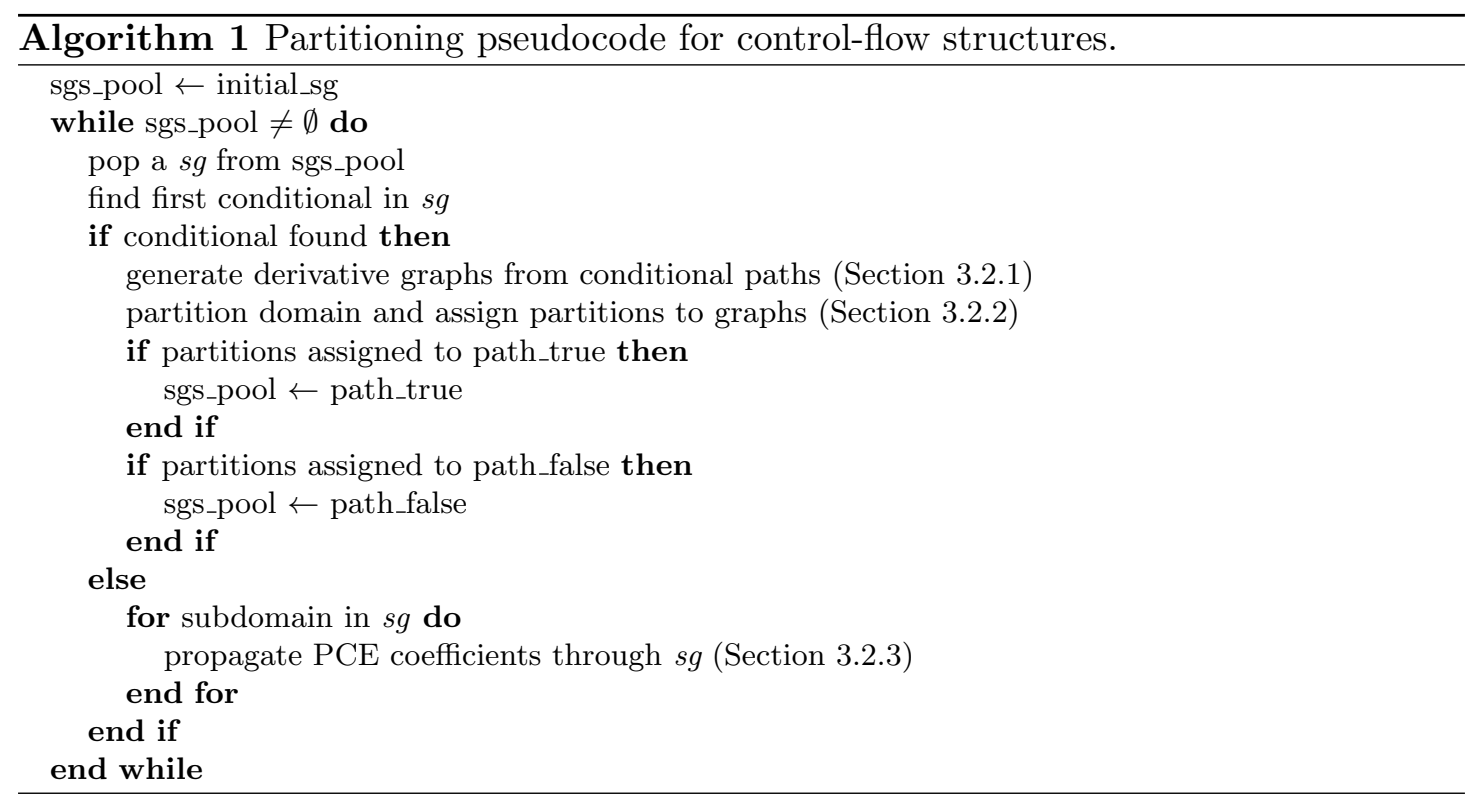

as explained in Section 3.2.2. We assign each resulting partition to its corresponding derivative subsystem. If a derivative subsystem ends up with no partitions assigned, we discard it. We repeat the process with all the newly generated subsystems and for each associated partition until there are no subsystems with control-flow structures left. Every time the process generates a subsystem without control-flow structures we can carry out a regular PCE analysis of dynamic range as detailed in Section 3.2.3, saving the results for the statistics computation stage.

\subsubsection{Control-flow structures}

We define a control-flow structure as a construct that selects the execution path to follow from a number of alternatives based on the outcome of a conditional statement. Control-flow structures always have the form of a conditional branch and one or more conditional assignments (often identified as a PHI node) that assign different values to a variable depending on the selected execution path. Even if a variable is only modified in one of the conditional branches, it will have an associated PHI node to determine whether the value after the join comes from that conditional branch or from the pre-branch block of code. Figure 3.1 shows how conditional structures are usually represented. It can be seen how the $\otimes$ operation in the upper section of the flow has successors in both execution paths, the conditional branch (BR block) directs the execution flow to Path 1 or 2 depending on the condition, and how the $\otimes$ in the lower block gets its value depending on the taken path.

Control-flow statements can present deterministic behaviour, where the result of the conditional statement is known beforehand, or random behaviour that depends on the input values to the system. The former case holds little interest for this section, since we can discard non selected choice paths and we can unroll loops with fixed number of iterations. In this aspect, deterministic control-flow structures are conceptually equivalent 


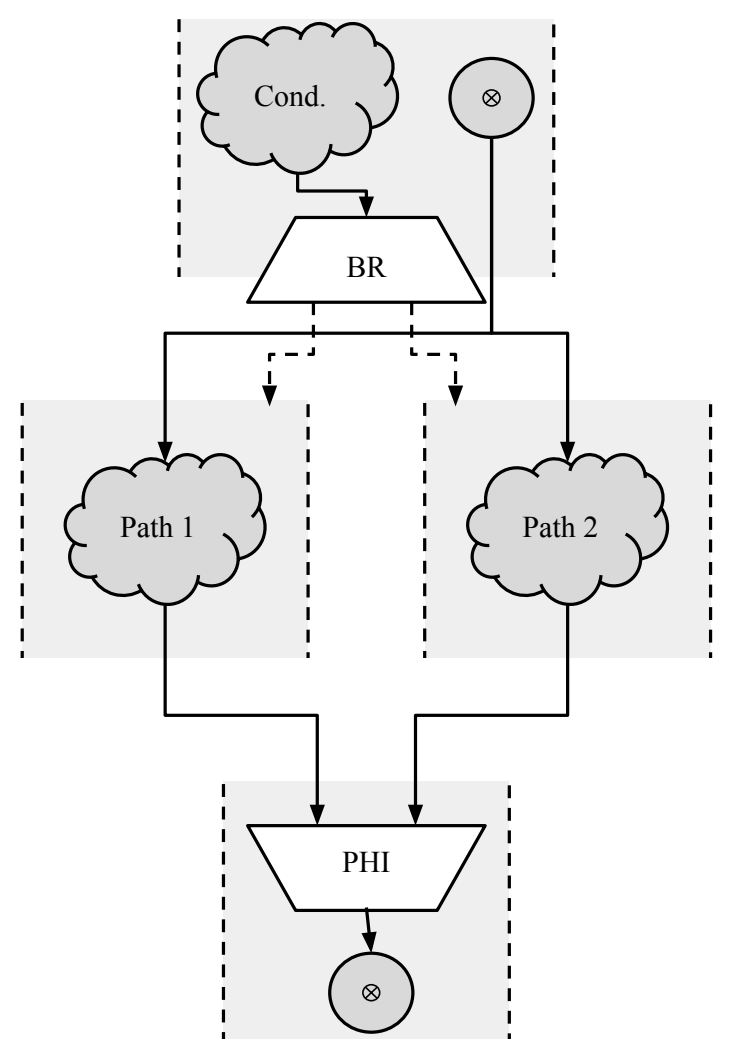

Fig. 3.1: Nodes representation of a control-flow structure.

to pure data-flow systems and we can study them following methods of already proven effectiveness [WZN06, Est11]. Instead, we focus on control-flow structures with random behaviour, in which the outcome of the conditional statements cannot be determined statically and thus all the possible execution paths have to be considered.

We distinguish between choice operators and loop structures. In both cases we can state that a control-flow structure with $D$ choices within a system $S$ leads to $i$ derivative systems $S_{i}$, being $0<i \leq 2 D$. In each of them, the whole control-flow structure is replaced by the $i$-th conditional execution path. In the following, we also assume that there are $N_{c f}$ control-flow structures in the system under study.

\section{Choice operators}

We define a choice operator as an operator for which every $S_{i}$ derivative subsystem has no more than $N_{c f}-1$ control-flow structures, meaning that none of the execution paths iterate over the same block of code containing the control-flow operation. The highlevel language instructions that generate this type of operator are if...then...else and switch...case. Without loss of generality, for the scope of this work we consider the latter as a concatenation of the former. Since our method is capable of dealing with any combination of nested and concatenated control-flow operators, this consideration holds valid.

The extraction of the execution paths is straightforward, as they correspond to each of 


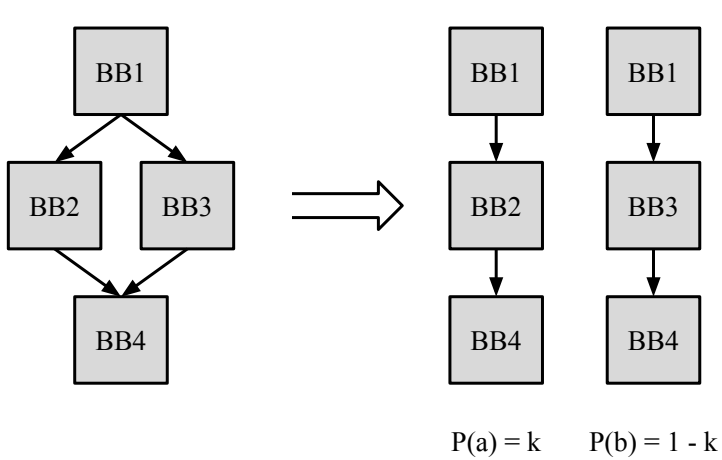

Fig. 3.2: Derivative execution paths from a choice operator.

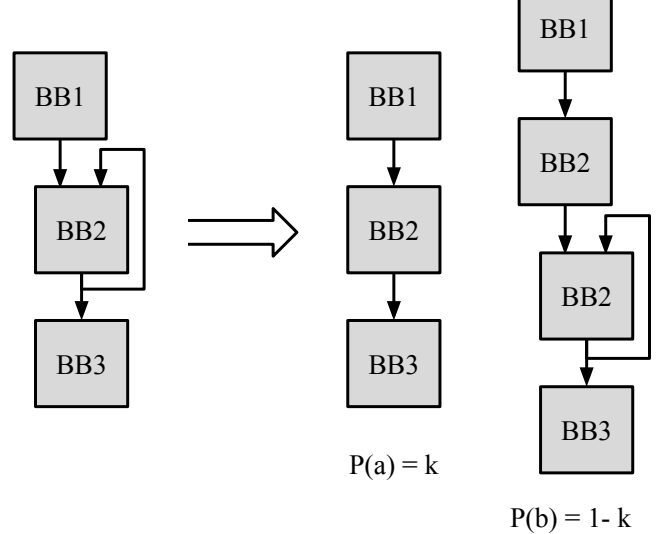

Fig. 3.3: Derivative execution paths from a loop structure.

the derivative $S_{i}$ subsystems. Figure 3.2 depicts the derivative execution paths generated from a choice operator.

\section{Loop structures}

If at least one of the $S_{i}$ subsystems contains $N_{c f}$ control-flow structures, the operator is a loop structure. One or more of the execution paths iterate over the same block of code, and we must process the subsystem further to reduce the number of controlflow structures. We identify the generation of this structure with the high-level language construct while... do. Using basic code transformations we can convert other constructs like for...loop and do... while to this type of construct.

We can safely assume that a loop structure has two possible paths: the next loop iteration and the break path, that continues with the next instruction after the construct. Thus, iteratively splitting the structure into derivative subsystems is equivalent to unrolling the loop. Figure 3.3 shows how this happens. Given that the halt condition is dynamic, we have to estimate when to consider the probability of a new iteration so small that it can be discarded. We control this as a function of the probability of the partition $J_{k}$ (see Equation 3.19), and we will see in Section 3.2.2 how to integrate it in the domain partitioning analysis so all the processing is done as homogeneously as 
possible.

We distinguish between two types of loops: On the one hand, those that are used to look for the convergence towards a value. The number of iterations in this type of loops is usually low, particularly in DSP systems where we process a stream of data. On the other hand, loops that iterate over data structures such as vectors and matrices. In this case, all the elements of the data structure could share the same quantization. The latter type of loops is not part of the scope of this Ph.D. Thesis.

\subsubsection{Domain partitioning}

The method we propose to perform the partitioning of the domain is based on the work presented in [WK05]. We adapt some of its methods and we keep our solution as in line as possible with the cited approach. To determine the regions of the domain that will make the system run through each of the two possible execution paths given a conditional instruction $I_{c}$, we consider $I_{c}=f\left(\tilde{u}_{l h s}, \tilde{u}_{r h s}, c m p\right)$, where $\tilde{u}_{l h s}$ and $\tilde{u}_{r h s}$ are the polynomials in PCE form corresponding to the two input expressions of the branch condition, and $c m p$ the relational operator of the condition. $I_{c}$ is thus described as shown in Equation 3.24.

$$
I_{c}= \begin{cases}1(\text { True }) & \text { if } \tilde{u}_{l h s} \text { cmp } \tilde{u}_{r h s} \\ 0(\text { False }) & \text { otherwise. }\end{cases}
$$

With this, we choose $k$ points $\tilde{\xi}_{i}, i=0,1, \cdots, k$, which are uniform grid points in $[-1,1]^{d}$, where $d$ is the number of dimensions (i.e. input random variables) of the system. In order to keep consistency with ME-gPC, we may use $k=N_{p}+1$, where $N_{p}+1$ is the size of the polynomial base. But this may cause some incorrect results if the size of the base is small. To avoid that we have determined empirically that $k=10\left(N_{p}+1\right)$ achieves results accurate enough for our purposes with negligible impact on the execution time regardless of the size of the base. We use these points to determine if the domain requires partitioning by solving

$$
\left(\sum_{i=0}^{k} I_{c}\left(\tilde{u}_{l h s}\left(\tilde{\xi}_{i}\right), \tilde{u}_{r h s}\left(\tilde{\xi}_{i}\right), c m p\right)\right) \bmod \left(N_{p}+1\right) .
$$

If the result of Equation 3.25 is zero, all $\tilde{\xi}_{i}$ lead to the same branch of the conditional and we can establish that the domain does not need to be partitioned. On the other hand, if the result is different from zero it means that parts of the domain belong to a branch of the conditional execution path and others to the other one, and thus a partition needs to be done.

When a domain is selected for partitioning, we split the domain $[-1,1]$ in two, $[-1,0]$ and $(0,1]$, for all the system variables and we re-scale them following the procedure presented in Section 3.1.4. We iteratively repeat the process with the resulting partitions until no further decomposition is required. A visual representation of a successful domain 


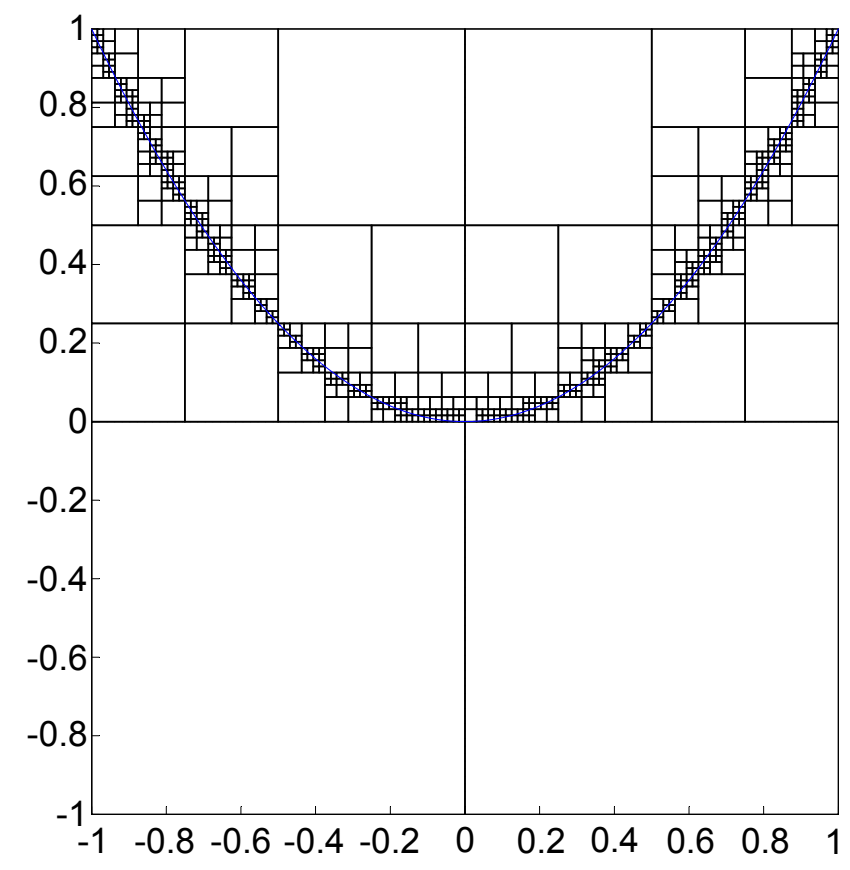

Fig. 3.4: Example of a decomposed domain.

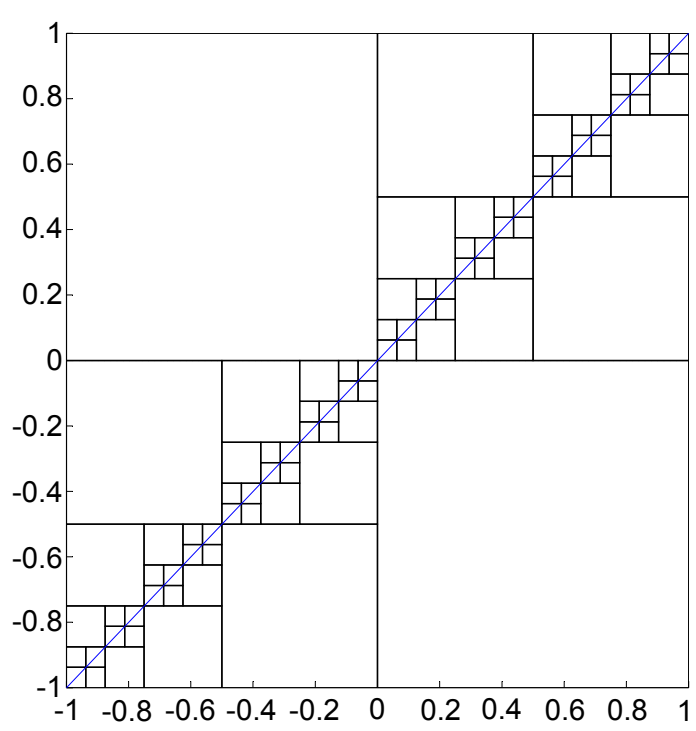

(a) $J_{\text {lim }}=10^{-3}$

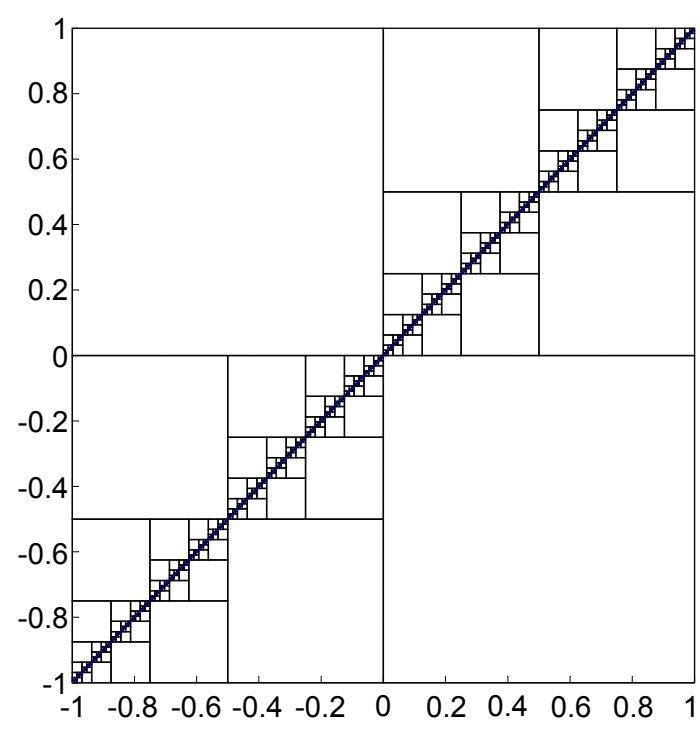

(b) $J_{\text {lim }}=10^{-5}$

Fig. 3.5: Domain decomposition for $x>y$ with different values of $J_{\text {lim }}$

partitioning for a conditional inequality $x^{2} \geq y$ can be observed in Figure 3.4.

This partitioning method does not guarantee a perfect fit between the generated partitions and the actual boundaries between two execution paths. Thus, we need to set an threshold for the domain decomposition in order to avoid an infinite number of iterations in the process. To this end, we define $J_{\text {lim }}$, a value function of $J_{k}$, which is defined as the associated probability of partition $k$. The value of $J_{l i m}$ establishes the minimum $J_{k}$ that a partition must have. For obvious reasons, the value of $J_{\text {lim }}$ will have 
an impact on the number of partitions performed, as well as on the accuracy of the results and the computation time. Designers must establish the value of $J_{l i m}$ considering the precision they expect from the modelling and the budgeted time they have for the process. Figure 3.5 presents the resulting decomposition of the domain $[-1,1]^{2}$ for the condition $x>y$ with different values of $J_{\text {lim }}$.

However, by doing this (i.e. stopping the partitioning process when $J_{\text {lim }}$ is reached) we are causing a number of partitions to have undefined outputs. Since not all $\tilde{\xi}_{i}$ point to the same execution branch in those partitions, we need to either decide the direction of the partitions or to process them further. For the scope of this project, we use a "majority vote" as the decision technique for choice operators: the direction with the largest number of points leading to it will be the chosen one. In case of draw, the direction is randomly decided.

\subsubsection{Propagation of the affine forms}

We propagate the PCE coefficients through the system in every iteration of the partitioning process in order to determine the outcome of the control-flow structures and to determine the actual system statistics once the partitioning finishes. In both cases, the system graphs through which we do the propagation are sequential (i.e. they do not contain control-flow structures), and we can apply the propagation rules of PCE. These rules exploit the orthogonality properties of the PCE polynomials to propagate the coefficients and capture dependencies between signals accurately. Table 3.3 summarizes the propagation rules through the basic arithmetic operations considering two inputs $\hat{x}$ and $\hat{y}$ defined as

$$
\begin{aligned}
& \hat{x}=\sum_{i=0}^{m-1} x_{i} \cdot \psi_{i}, \\
& \hat{y}=\sum_{i=0}^{m-1} y_{i} \cdot \psi_{i},
\end{aligned}
$$

and where $A$ is a $m \times m$ matrix and $X, Y$ and $Z$ are column vectors containing the coefficients for $\hat{x}, \hat{y}$ and $\hat{z}$, respectively. The interested reader can find the procedure and mathematical background for these rules in [WZN06, $\left.\mathrm{DNP}^{+} 04\right]$.

\section{Propagation example}

We illustrate the propagation of PCE coefficients with a multiply-accumulate operation $\operatorname{mac}(a, b, c)=a+(b \cdot c)$. For this system we use a second-order third-dimension Legendre PCE.

The computation is as follows. We associate each input to an independent RV and represent it as a first-order Legendre polynomial: 
Table 3.3: Coefficient propagation rules of PCE basic operations.

\begin{tabular}{|c|c|}
\hline Operation & Coefficient propagation rule \\
\hline \hline Addition & $z_{i}=x_{i}+y_{i}$ \\
\hline Subtraction & $z_{i}=x_{i}-y_{i}$ \\
\hline Constant multiplication & $z_{i}=c \cdot y_{i}$ \\
\hline Non-linear multiplication & $z_{k}=\sum_{i=0}^{m-1} \sum_{j=1}^{m} C(i, j, k) x_{i} y_{i}$ \\
\hline Non-linear division & $Z=A^{-1} \cdot X \Rightarrow A=C \cdot Y$ \\
\hline
\end{tabular}

$$
\begin{aligned}
& \hat{a}=a_{0}+a_{1} \epsilon_{a}, \\
& \hat{b}=b_{0}+b_{1} \epsilon_{b}, \\
& \hat{c}=c_{0}+c_{1} \epsilon_{c},
\end{aligned}
$$

where $\epsilon_{i}$ are uniformly distributed RVs in the interval $[-1,1]$ Since the multiplication is not linear, we need a second order PCE which, for a Legendre basis, includes the following terms:

Constant value: 1 ,

First order: $\epsilon_{a}, \epsilon_{b}, \epsilon_{c}$,

Second order: $\frac{1}{2}\left(3 \epsilon_{a}^{2}-1\right), \frac{1}{2}\left(3 \epsilon_{b}^{2}-1\right), \frac{1}{2}\left(3 \epsilon_{c}^{2}-1\right)$,

Cross terms: $\epsilon_{a} \epsilon_{b}, \epsilon_{a} \epsilon_{c}, \epsilon_{b} \epsilon_{c}$,

where, in practice, not all the parameters in the polynomials need to be different from 0 to represent the output variables. Following the rules in Table 3.3 we obtain the intermediate value $\hat{m}$ and the output value $\hat{m a c}$ :

$$
\begin{gathered}
\hat{m}=\hat{b} \cdot \hat{c}=b_{0} c_{0}+c_{0} b_{1} \epsilon_{b}+b_{0} c_{1} \epsilon_{c}+b_{1} c_{1} \epsilon_{b} \epsilon_{c}, \\
\hat{m a c}=\hat{a}+\hat{m}=a_{0}+a_{1} \epsilon_{a}+b_{0} c_{0}+c_{0} b_{1} \epsilon_{b}+b_{0} c_{1} \epsilon_{c}+b_{1} c_{1} \epsilon_{b} \epsilon_{c} .
\end{gathered}
$$

\subsubsection{Computation of the system statistics}

To compute the statistical moments of the signals in the system we need to reconstruct it from the partial results of the propagations through the different partitions of the domain. We do this applying Equation 3.23. We consider the PCE expansion of signal $y$ in the $i$-th partition:

$$
\hat{y}_{i}=y_{i, 0}+\sum_{j=1}^{m} y_{i, j} \psi_{i, j},
$$

where the terms $y_{i, j}$ are the coefficients of the PCE and $\psi_{i, j}$ are the terms of the polynomial base. Given an associated probability $J_{k, i}$ for the partition, the mean or first order 


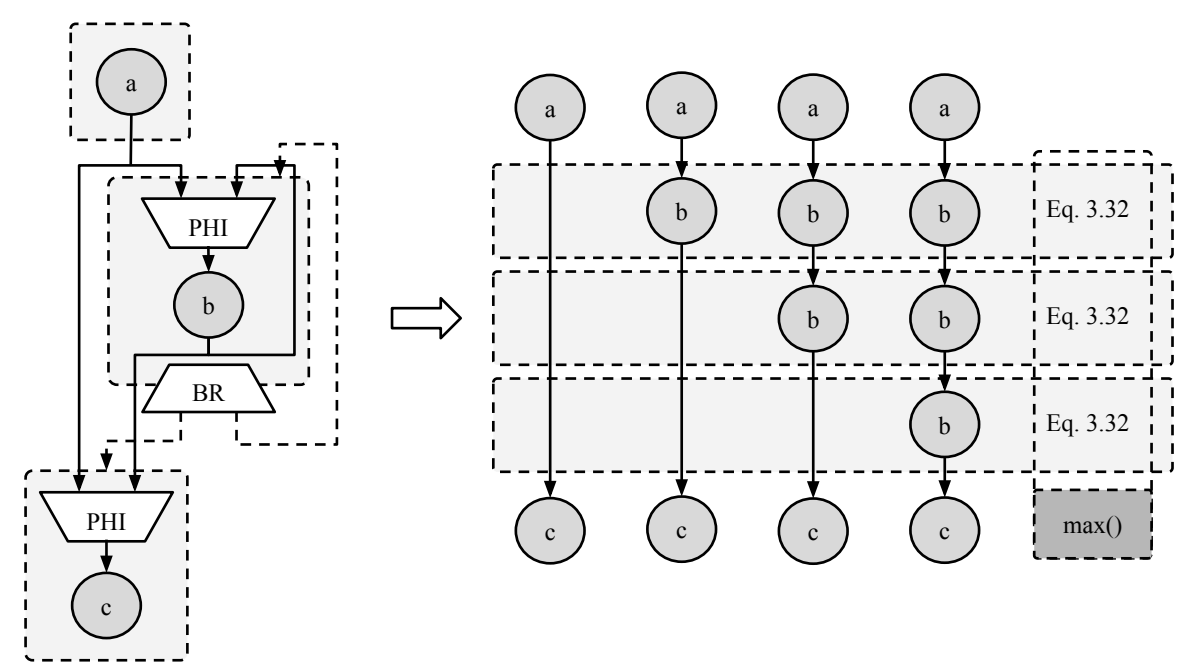

Fig. 3.6: Dynamic range of an intermediate variable across an unrolled loop.

moment is $\langle y\rangle=\sum_{i=0}^{p-1} y_{i, 0} J_{k, i}$, being $p$ the total number of partitions. To calculate the second order moment of $y$ we take advantage of the orthogonality of the polynomials and we use the following formula:

$$
\sigma_{y}^{2}=\left(\sum_{i=0}^{p-1}\left(J_{k, i}\left(\sum_{j=0}^{m_{i}}\left(y_{i, j}^{2}<\psi_{i}^{2}>\right)\right)-<y>^{2}\right)\right) .
$$

The equations above are applicable to both outputs and intermediate signals. It must be noted, though, that intermediate signals may not appear in every execution path and, thus, not in every partition. Whenever this happens, we will consider that the values of all the $y_{i, j}$ coefficients for that variable are equal to zero. Also, we must consider the opposite situation: when unrolling a loop, the same original signal will appear several times in the derivative DFGs. When this happens, in order to estimate the dynamic range of the original signal we will have to apply Equation 3.32 across all the appearances for each iteration of the unrolled loop, and then get the maximum value (i.e. the value that requires the largest dynamic range bits assignment) of the results, as shown in Figure 3.6

\subsubsection{Example: Absolute value of a multiplication}

In this section we apply our proposed methodology to model the dynamic range of the absolute value of the multiplication of two variables $x$ and $y$, both of them with distributions $U[-1,1]$. We approach the absolute value as a conditional structure where we invert the sign of the addition result if it is negative. The diagram on the left side of Figure 3.7 shows the structure of the system.

The PCE polynomials for each input of the system are 

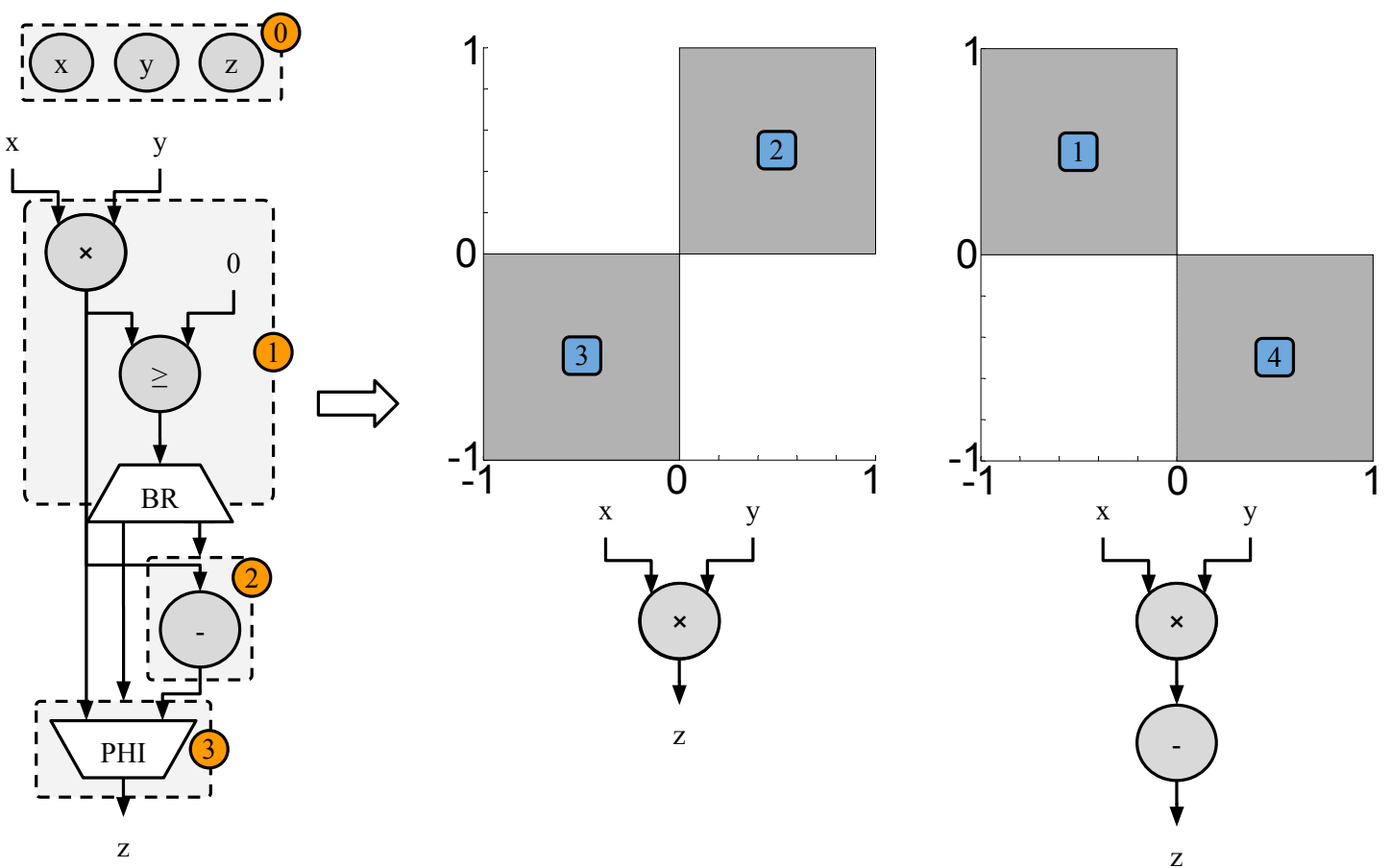

Fig. 3.7: Absolute value operation and execution paths corresponding to sub-domains.

Table 3.4: PCE polynomials for sub-domain inputs in absolute value.

\begin{tabular}{|c|c|c|}
\hline Partition & PCE expansion & $J_{k}$ \\
\hline \hline 1 & $\begin{array}{c}\hat{x}_{1}=-0.5+0.5 \epsilon_{0}^{1} \\
\hat{y}_{1}=0.5+0.5 \epsilon_{1}^{1}\end{array}$ & 0.25 \\
\hline 2 & $\hat{x}_{2}=0.5+0.5 \epsilon_{0}^{2}$ & 0.25 \\
& $\hat{y}_{2}=0.5+0.5 \epsilon_{1}^{2}$ & \\
\hline \multirow{2}{*}{3} & $\hat{x}_{3}=-0.5+0.5 \epsilon_{0}^{3}$ & 0.25 \\
& $\hat{y}_{3}=-0.5+0.5 \epsilon_{1}^{3}$ & \\
\hline \multirow{2}{*}{4} & $\hat{x}_{4}=0.5+0.5 \epsilon_{0}^{4}$ & 0.25 \\
\hline
\end{tabular}

$$
\begin{aligned}
& \hat{x}=\epsilon_{0} \\
& \hat{y}=\epsilon_{1},
\end{aligned}
$$

where $\epsilon_{1}$ and $\epsilon_{2}$ are RVs $U[-1,1]$. After obtaining the two possible execution paths of the system, we study the conditional operation from Basic Block 1. Following the PCE propagation rules, the left-hand side of the conditional operator is

$$
\hat{x} \hat{y}=\epsilon_{0} \epsilon_{1} .
$$

We apply Algorithm 1 to obtain four partitions and, according to the outcome of each evaluation, we associate them to the corresponding execution path. The right side 
of Figure 3.7 shows the correspondence between execution paths and sub-domains. Table 3.4 presents the resized coefficients for the inputs and the probability $J_{k}$ of each subdomain, where, $\hat{x}_{i}$ and $\hat{y}_{i}$ are the PCE polynomials of the input variables $x$ and $y$ for the $i$-th partition.

Then, we propagate the coefficients of each partition through the corresponding DFG:

$$
\begin{gathered}
\hat{x}_{1} \hat{y}_{1}=-0.25+0.25 \epsilon_{0}^{1}-0.25 \epsilon_{1}^{1}+0.25 \epsilon_{0}^{1} \epsilon_{1}^{1}, \\
\hat{z}_{1}=0.25-0.25 \epsilon_{0}^{1}+0.25 \epsilon_{1}^{1}-0.25 \epsilon_{0}^{1} \epsilon_{1}^{1}, \\
\hat{z}_{2}=0.25+0.25 \epsilon_{0}^{2}+0.25 \epsilon_{1}^{2}+0.25 \epsilon_{0}^{2} \epsilon_{1}^{2}, \\
\hat{z}_{3}=0.25-0.25 \epsilon_{0}^{3}-0.25 \epsilon_{1}^{3}+0.25 \epsilon_{0}^{3} \epsilon_{1}^{3}, \\
\hat{x}_{4} \hat{y}_{4}=-0.25-0.25 \epsilon_{0}^{4}+0.25 \epsilon_{1}^{4}+0.25 \epsilon_{0}^{4} \epsilon_{1}^{4}, \\
\hat{z}_{4}=0.25+0.25 \epsilon_{0}^{4}-0.25 \epsilon_{1}^{4}-0.25 \epsilon_{0}^{4} \epsilon_{1}^{4} .
\end{gathered}
$$

With the four different $\hat{z}_{i}$ expansions, we can compute the operation statistics following the equations in Section 3.2.4. This gives us a mean value 0.25 and a variance 0.0486 , values that match perfectly those obtained by Monte-Carlo simulation with $10^{7}$ samples.

\subsection{Round-off noise modelling in systems with control- flow structures}

Once the partitioning process outlined in Algorithm 1 is performed, each of the resulting derivative Data Flow Graphs is a linear sequence of arithmetic operations. As such, we can model the RON of each individual DFG separately and then combine the results to obtain the statistical moments of the system as a whole.

In this section we describe the process to compute the RON in systems with controlflow structures. The process, summarized in Figure 3.8, starts by considering each of the derivative DFGs generated while modelling the dynamic range of the system. We add the required noise input RVs to the DFG and, after generating the corresponding orthogonal basis and pre-computed structures, we propagate the coefficients according to the rules described in Table 3.3. Finally, we process and combine the partial results to obtain the statistical moments of the complete quantized system.

It must be noted that, since we inject the noise sources for RON modelling after the analysis of the control-flow structures, the effects of RON on decision operators are not taken into account. This topic, not covered in this Ph.D. Thesis, has been studied by other authors. In essence, the conclusion is that those systems where the effects of RON on decision operators can have critical consequences on the system's behaviour 


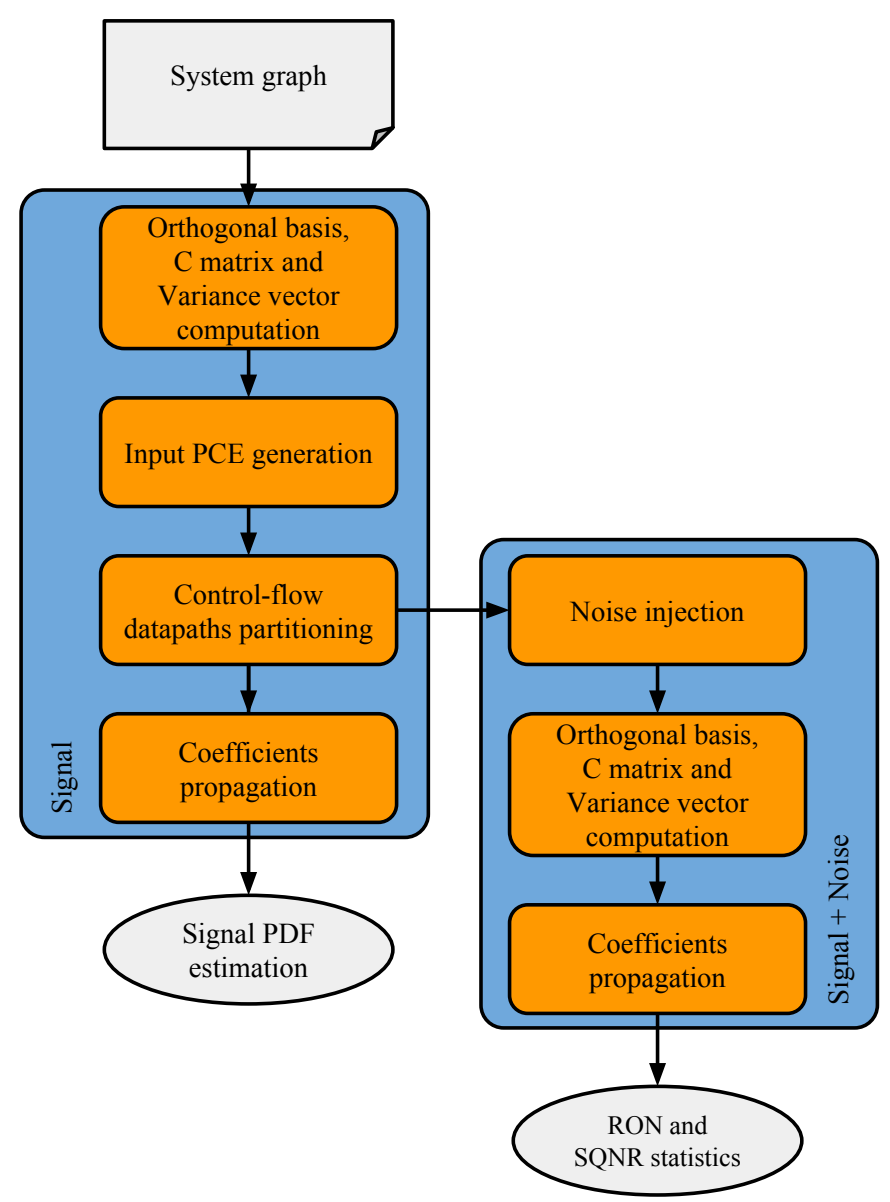

Fig. 3.8: Methodology for RON computation in systems with control-flow structures.

are systems where, to begin with, no quantization should be applied on the variables involved. If the effects are non critical, then the impact of including RON after modelling the control-flow structures becomes a second order effect that can be controlled by appropriately setting slightly more conservative global noise requirements. The interested reader can find more information on the topic in [SB04b, PRMS10a].

\subsubsection{Modelling of the quantization noise sources}

We model the quantization of the signals using the Discrete Noise Model (DNM), presented in [CCL99] and extended in [LCCNT08]. With this model, the statistical moments of the noise input are

$$
m_{n_{i}}=-\frac{2^{-f_{i q}}-2^{-f_{i}}}{2} \gamma, \sigma_{n_{i}}^{2}=\frac{2^{-2 f_{i q}}+(-\gamma) 2^{-2 f_{i}+(1-\gamma)}}{12},
$$

being $f_{i q}$ the used fractional word-length, $f_{i}$ the minimum number of fractional bits required to represent the signal $i$ without loss of precision and $\gamma$ a parameter to determine if we apply the quantization by truncation $(\gamma=1)$ or rounding $(\gamma=0)$. In the case of the rounding quantisers, the equation above assumes $f_{i}>f_{i q}$. Otherwise, $\sigma_{n_{i}}^{2}=0$. We 


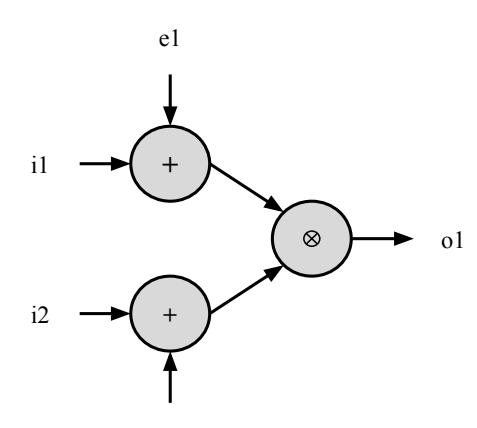

e2

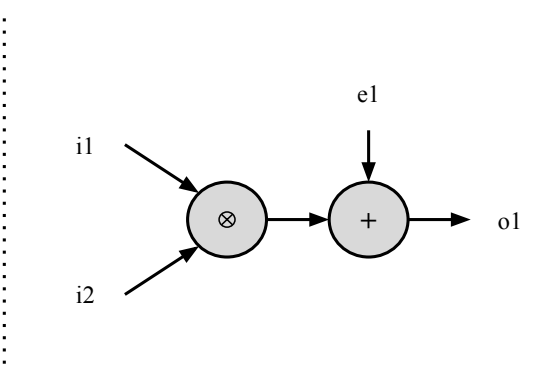

Fig. 3.9: Two different ways of introducing AWN sources to an operation.

introduce the noise sources in the system adding them to the edges that connect the operations in the Data Flow Graph of the system to analyse.

Figure 3.9 presents two different ways of quantizing an operator $\otimes(i 1, i 2)=o 1$. Each noise source introduces a new random variable $e_{i}$ to the system, thus incrementing the size of the input stochastic space and potentially slowing down the PCE propagation to the outputs. By adding a quantization to each operation input (Figure 3.9, left) we can generate more optimized circuits, as each successor to a given output will have its own word-length, while adding the noise source to the output (Figure 3.9, right) makes all successors to share the same size. Thus, the former approach will take more time to generate a RON model but will provide better results, while the latter provides rougher optimizations in shorter execution times. The methodology we present in this chapter is valid for both approaches, so the decision is left to the designer and will depend on the requisites and resources of the quantization process.

While introducing the noise sources in the system we must pay attention to those operations that are repeated across derivative DFGs and along unrolled iterations of loop structures. We need to assign the same $f_{w q}$ value across all appearances of nodes that correspond to the original system graph operation. Otherwise, the word-length assignments would not be consistent with the modelled system statistics. In Figure 3.10 we show two control-flow structures, and how to assign fractional word-lengths to each of them. The top one is a generic choice operator, that generates a derivative sub-system for each of the two possible outcomes of the condition, while in the bottom one we have the same loop structure as in Figure 3.6, which unrolls three times and generates as many derivative sub-systems. For the sake of clarity, we only add the noise source $q_{i}$ to the output of each node $i$. We represent the addition of these noise sources with dark grey nodes. On the right side of both cases in the figure, fine dotted lines indicate that the noise source nodes connected by them need to have the same $f_{w q}$ assigned to them.

\subsubsection{RON coefficients propagation example}

As we indicated before, the rules for propagating the PCE coefficients through the system are not different from the ones we described in Section 3.2.3. For the sake of 

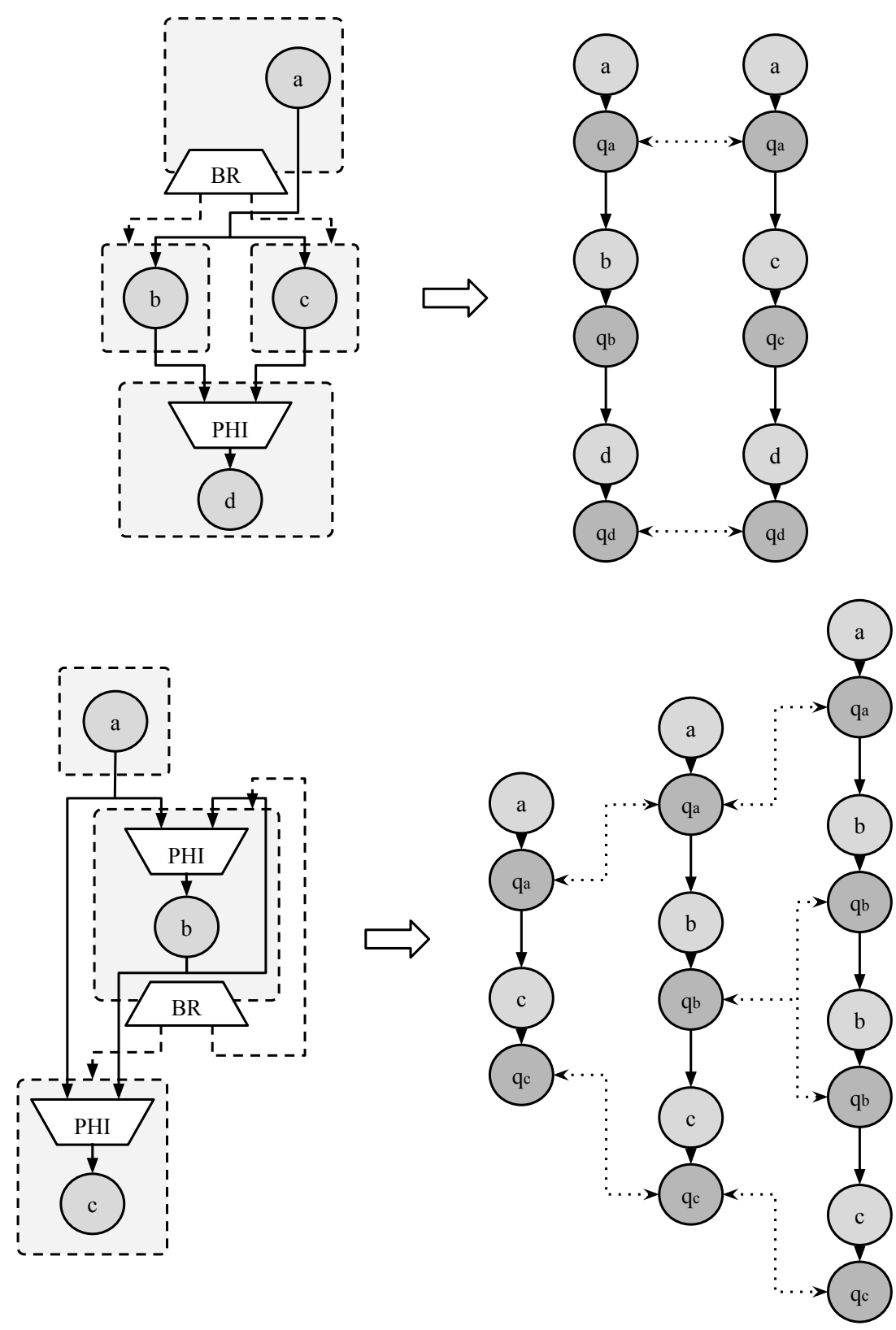

Fig. 3.10: Fractional word-length to derivative DFGs nodes assignment examples.

clarity we present here the propagation of PCE terms through a quantized version of the multiply-taccumulate operation $\operatorname{mac}(a, b, c)=a+(b \cdot c)$ we used in Section 3.2.3. Also, in order to keep the example simple, we use rounding quantizers $(\gamma=0)$ and we quantize the operation as a fused multiply-add. Considering that each signal is quantized to $f_{w q a}$, $f_{w q b}, f_{w q c}$, and $f_{w q m a c}$, we represent each noise source as:

$$
\hat{n_{i}}=\frac{2^{-f_{q w i}}}{2} \epsilon_{n i}=n_{i} \epsilon_{n i}, \quad i=a, b, c, m a c,
$$

being $\epsilon_{n i}$ uniformly distributed RVs in $[-1,1]$. Considering both signal and noise inputs, we can construct a new second order PCE for the system with the following terms: 


\section{CHAPTER 3. QUANTIZATION OF SYSTEMS WITH CONTROL FLOW STRUCTURES}

Table 3.5: Propagation of signal and RON terms through a MAC operation.

\begin{tabular}{|c|c|}
\hline Operation & Result \\
\hline \hline$\hat{a_{q}}=\hat{a}+\hat{n_{a}}$ & $a_{0}+a_{1} \epsilon_{a}+n_{a} \epsilon_{n a}$ \\
\hline$\hat{b_{q}}=\hat{b}+\hat{n_{b}}$ & $b_{0}+b_{1} \epsilon_{b}+n_{b} \epsilon_{n b}$ \\
\hline$\hat{c_{q}}=\hat{c}+\hat{n_{c}}$ & $c_{0}+c_{1} \epsilon_{c}+n_{c} \epsilon_{n c}$ \\
\hline$\hat{m}=\hat{b_{q}} \cdot \hat{c_{q}}$ & $b_{0} c_{0}+b_{0} c_{1} \epsilon_{c}+b_{0} n_{c} \epsilon_{n c}+c_{0} b_{1} \epsilon_{b}+c_{1} b_{1} \epsilon_{b} \epsilon_{c}+$ \\
& $+n_{c} b_{1} \epsilon_{b} \epsilon_{n c}+c_{0} n_{b} \epsilon_{n b}+c_{1} n_{b} \epsilon_{n b} \epsilon_{c}+n_{c} n_{b} \epsilon_{n b} \epsilon_{n c}$ \\
\hline$\hat{m a c}=\hat{a_{q}}+\hat{m}$ & $a_{0}+a_{1} \epsilon_{a}+n_{a} \epsilon_{n a}+b_{0} c_{0}+b_{0} c_{1} \epsilon_{c}+b_{0} n_{c} \epsilon_{n c}+c_{0} b_{1} \epsilon_{b}+$ \\
& $+c_{1} b_{1} \epsilon_{b} \epsilon_{c}+n_{c} b_{1} \epsilon_{b} \epsilon_{n c}+c_{0} n_{b} \epsilon_{n b}+c_{1} n_{b} \epsilon_{n b} \epsilon_{c}+n_{c} n_{b} \epsilon_{n b} \epsilon_{n c}$ \\
\hline$m \hat{a} c_{q}=\hat{m a c}+\hat{n_{m a c}}$ & $a_{0}+a_{1} \epsilon_{a}+n_{a} \epsilon_{n a}+b_{0} c_{0}+b_{0} c_{1} \epsilon_{c}+b_{0} n_{c} \epsilon_{n c}+c_{0} b_{1} \epsilon_{b}+$ \\
& $+c_{1} b_{1} \epsilon_{b} \epsilon_{c}+n_{c} b_{1} \epsilon_{b} \epsilon_{n c}+c_{0} n_{b} \epsilon_{n b}+c_{1} n_{b} \epsilon_{n b} \epsilon_{c}+n_{c} n_{b} \epsilon_{n b} \epsilon_{n c}+n_{m a c} \epsilon_{n m a c}$ \\
\hline
\end{tabular}

Constant value: 1 ,

First order: $\epsilon_{a}, \epsilon_{b}, \epsilon_{c}, \epsilon_{n a}, \epsilon_{n b}, \epsilon_{n c}, \epsilon_{n m a c}$,

Second order: $\frac{1}{2}\left(3 \epsilon_{a}^{2}-1\right), \frac{1}{2}\left(3 \epsilon_{b}^{2}-1\right), \frac{1}{2}\left(3 \epsilon_{c}^{2}-1\right), \frac{1}{2}\left(3 \epsilon_{n a}^{2}-1\right), \frac{1}{2}\left(3 \epsilon_{n b}^{2}-1\right), \frac{1}{2}\left(3 \epsilon_{n c}^{2}-1\right)$, $\frac{1}{2}\left(3 \epsilon_{n m a c}^{2}-1\right)$,

Cross terms: $\epsilon_{a} \epsilon_{b}, \epsilon_{a} \epsilon_{c}, \epsilon_{b} \epsilon_{c}, \epsilon_{a} \epsilon_{n a}, \epsilon_{a} \epsilon_{n b}, \epsilon_{a} \epsilon_{n c}, \epsilon_{a} \epsilon_{n m a c}, \epsilon_{b} \epsilon_{n a}, \epsilon_{b} \epsilon_{n b}, \epsilon_{b} \epsilon_{n c}, \epsilon_{b} \epsilon_{n m a c}$ $\epsilon_{c} \epsilon_{n a}, \epsilon_{c} \epsilon_{n b}, \epsilon_{c} \epsilon_{n c}, \epsilon_{c} \epsilon_{n m a c}, \epsilon_{n a} \epsilon_{n b}, \epsilon_{n a} \epsilon_{n c}, \epsilon_{n a} \epsilon_{n m a c}, \epsilon_{n b} \epsilon_{n c}, \epsilon_{n b} \epsilon_{n m a c}, \epsilon_{n c} \epsilon_{n m a c}$.

After propagating the coefficients through the operations, we obtain the results shown in Table 3.5. It can be appreciated how signal and noise sources are clearly separated in the final MAA form. We must take into account that, in the case of truncation quantizers $(\gamma=1)$ the constant term will be affected not only by signal propagations, but also by noise. In this case, we need to identify the noise contribution to the term, something that we can achieve by comparing it to the values resulting from modelling the system without noise inputs. Since that information is available from the dynamic range modelling stage, the computational overload is negligible.

\subsubsection{Computation of the RON statistics}

Since the approach for modelling RON is homogeneous with the one for estimating dynamic ranges, in order to calculate the statistical moments of the output signals we use the same formulations as in Section 3.2.4.

Using this procedure we calculate the variance of the system outputs both with and without considering the RON sources. An advantage of PCE polynomials is that the noise terms are separated from the signal terms. We can obtain higher order moments if needed through Equation 3.23 and applying the rules described, for example, in [GS91].

Once we know the signal and RON variances, we can determine several of the quantization noise metrics we reviewed in Section 2.2.3. In this Ph.D. thesis we use the ratio 


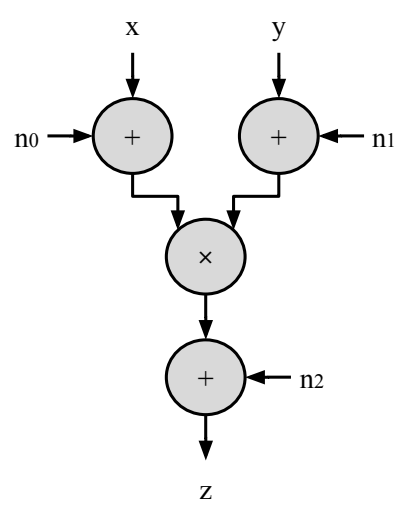

23
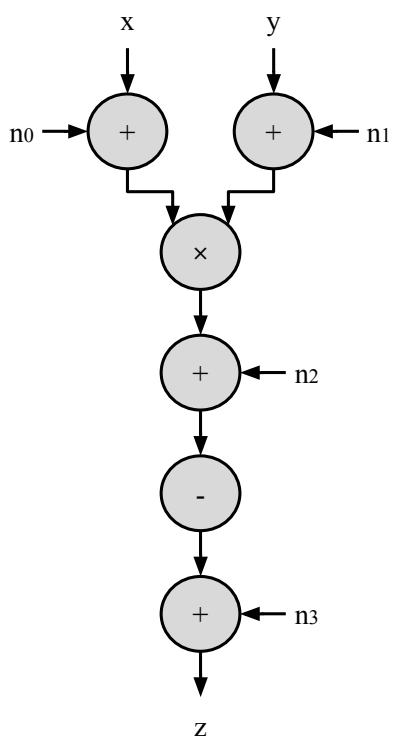

1

Fig. 3.11: Absolute value example paths with noise sources.

between variances as an approximation to the SQNR, as it has been applied in several other works $\left[\mathrm{CCC}^{+} 06\right]$ :

$$
S Q N R \simeq 10 \cdot \log _{10}\left(\frac{\sigma_{y}^{2}}{\sigma_{R O N}^{2}}\right) .
$$

PCE polynomials also allow us to obtain easily the MM value, sometimes used as noise metric. The MM value is calculated as

$$
M M_{y}=\max \left(M M_{y, 0}, M M_{y, 1}, \cdots, M M_{y, p-1}\right),
$$

where we define each $M M_{y, i}$ as the maximum mismatch in the $i$-th partition:

$$
M M_{y, i}=\sum_{j=0}^{m}\left[y_{i, j} \mid y_{i, j} \text { is a noise term }\right] .
$$

This calculation is possible because of the orthonormal property of the polynomial base. Also, it shows the flexibility of PCE polynomial representations for RON analysis and modelling.

\subsubsection{Example: Quantization of absolute value of a multiplication}

In Section 3.2.5 we have estimated the dynamic range of the absolute value of a multiplication, approaching the absolute value as a conditional structure. Now, we continue that example applying the RON modelling technique we have described in this Section to that operation. Following the steps summarized in Figure 3.8, we start from the two derivative DFGs depicted in Figure 3.7 and we add noise sources to them. 


\section{CHAPTER 3. QUANTIZATION OF SYSTEMS WITH CONTROL FLOW STRUCTURES}

The noise source PCE polynomials are calculated according to Equation 3.39. The first thing to consider here is that several elements in the system are repeated across both derivative DFGs. In this case, both $x$ and $y$ inputs, and the product operation. Thus, we have to match the $f_{w q}$ assignments for each of them. The resulting PCE polynomials for the noise inputs are, then,

$$
\begin{gathered}
\hat{n}_{i}=\frac{2^{-f_{i}}}{2} \epsilon_{n i}, i=0,1 \\
\hat{n}_{2}=\left(\frac{2^{-f_{2}}}{2}+\frac{2^{-2 f_{i}+1}}{2}\right) \epsilon_{n 2} \approx \frac{2^{-f_{2}}}{2} \epsilon_{n 2} . \\
\hat{n}_{3}=0
\end{gathered}
$$

It must be noted how, since each derivative subsystem is studied independently, we can re-use the same RVs for noise sources that do not belong to the same DFG. We use the polynomials in Equation 3.44 to complete the information of the inputs for each partition of the input domain. Since the domain for each of the noise RVs is not partitioned at any time, the $J_{k}$ of each partition remains the same as the one we calculated during the partitioning process in Section 3.2.5. Then, we propagate the coefficients of each partition through the corresponding data flow graph:

$$
\begin{aligned}
& \hat{a}_{1}=\hat{x}_{1}+\hat{n}_{0}=-0.5+0.5 \epsilon_{0}^{1}+\frac{2^{-f_{0}}}{2} \epsilon_{n 0}, \\
& \hat{b}_{1}=\hat{y}_{1}+\hat{n}_{1}=0.5+0.5 \epsilon_{1}^{1}+\frac{2^{-f_{1}}}{2} \epsilon_{n 1}, \\
& \hat{c}_{1}=\hat{a}_{1} \hat{b}_{1}=-0.25+0.25 \epsilon_{0}^{1}-0.25 \epsilon_{1}^{1}+\frac{2^{-f_{0}}}{4} \epsilon_{n 0}-\frac{2^{-f_{1}}}{4} \epsilon_{n 1}+0.25 \epsilon_{0}^{1} \epsilon_{1}^{1}+ \\
& +\frac{2^{-f_{1}}}{4} \epsilon_{0}^{1} \epsilon_{n 1}+\frac{2^{-f_{0}}}{4} \epsilon_{1}^{1} \epsilon_{n 0}+\frac{2^{-f_{0}}}{2} \frac{2^{-f_{1}}}{2} \epsilon_{n 0} \epsilon_{n 1}, \\
& \hat{d}_{1}=\hat{c}_{1}+\hat{n}_{2}=-0.25+0.25 \epsilon_{0}^{1}-0.25 \epsilon_{1}^{1}+\frac{2^{-f_{0}}}{4} \epsilon_{n 0}-\frac{2^{-f_{1}}}{4} \epsilon_{n 1}+\frac{2^{-f_{2}}}{2} \epsilon_{n 2}+ \\
& +0.25 \epsilon_{0}^{1} \epsilon_{1}^{1}+\frac{2^{-f_{1}}}{4} \epsilon_{0}^{1} \epsilon_{n 1}+\frac{2^{-f_{0}}}{4} \epsilon_{1}^{1} \epsilon_{n 0}+\frac{2^{-f_{0}}}{2} \frac{2^{-f_{1}}}{2} \epsilon_{n 0} \epsilon_{n 1}, \\
& \hat{z}_{1}=-\hat{d}_{1}=0.25-0.25 \epsilon_{0}^{1}+0.25 \epsilon_{1}^{1}-\frac{2^{-f_{0}}}{4} \epsilon_{n 0}+\frac{2^{-f_{1}}}{4} \epsilon_{n 1}-\frac{2^{-f_{2}}}{2} \epsilon_{n 2}- \\
& -0.25 \epsilon_{0}^{1} \epsilon_{1}^{1}-\frac{2^{-f_{1}}}{4} \epsilon_{0}^{1} \epsilon_{n 1}-\frac{2^{-f_{0}}}{4} \epsilon_{1}^{1} \epsilon_{n 0}-\frac{2^{-f_{0}}}{2} \frac{2^{-f_{1}}}{2} \epsilon_{n 0} \epsilon_{n 1}, \\
& \hat{a}_{2}=\hat{x}_{2}+\hat{n}_{0}=0.5+0.5 \epsilon_{0}^{2}+\frac{2^{-f_{0}}}{2} \epsilon_{n 0}, \\
& \hat{b}_{2}=\hat{y}_{2}+\hat{n}_{1}=0.5+0.5 \epsilon_{1}^{2}+\frac{2^{-f_{1}}}{2} \epsilon_{n 1}, \\
& \hat{c}_{2}=\hat{a}_{2} \hat{b}_{2}=0.25+0.25 \epsilon_{0}^{2}+0.25 \epsilon_{1}^{2}+\frac{2^{-f_{0}}}{4} \epsilon_{n 0}+\frac{2^{-f_{1}}}{4} \epsilon_{n 1}+0.25 \epsilon_{0}^{2} \epsilon_{1}^{2}+ \\
& +\frac{2^{-f_{1}}}{4} \epsilon_{0}^{2} \epsilon_{n 1}+\frac{2^{-f_{0}}}{4} \epsilon_{1}^{2} \epsilon_{n 0}+\frac{2^{-f_{0}}}{2} \frac{2^{-f_{1}}}{2} \epsilon_{n 0} \epsilon_{n 1}, \\
& \hat{z}_{2}=\hat{c}_{2}+\hat{n}_{2}=0.25+0.25 \epsilon_{0}^{2}+0.25 \epsilon_{1}^{2}+\frac{2^{-f_{0}}}{4} \epsilon_{n 0}+\frac{2^{-f_{1}}}{4} \epsilon_{n 1}+\frac{2^{-f_{2}}}{2} \epsilon_{n 2}+ \\
& +0.25 \epsilon_{0}^{2} \epsilon_{1}^{2}+\frac{2^{-f_{1}}}{4} \epsilon_{0}^{2} \epsilon_{n 1}+\frac{2^{-f_{0}}}{4} \epsilon_{1}^{2} \epsilon_{n 0}+\frac{2^{-f_{0}}}{2} \frac{2^{-f_{1}}}{2} \epsilon_{n 0} \epsilon_{n 1},
\end{aligned}
$$




$$
\begin{aligned}
& \hat{a}_{3}=\hat{x}_{3}+\hat{n}_{0}=-0.5+0.5 \epsilon_{0}^{3}+\frac{2^{-f_{0}}}{2} \epsilon_{n 0}, \\
& \hat{b}_{3}=\hat{y}_{3}+\hat{n}_{1}=-0.5+0.5 \epsilon_{1}^{3}+\frac{2^{-f_{1}}}{2} \epsilon_{n 1} \text {, } \\
& \hat{c}_{3}=\hat{a}_{3} \hat{b}_{3}=0.25-0.25 \epsilon_{0}^{3}-0.25 \epsilon_{1}^{3}-\frac{2^{-f_{0}}}{4} \epsilon_{n 0}-\frac{2^{-f_{1}}}{4} \epsilon_{n 1}+0.25 \epsilon_{0}^{3} \epsilon_{1}^{3}+ \\
& +\frac{2^{-f_{1}}}{4} \epsilon_{0}^{3} \epsilon_{n 1}+\frac{2^{-f_{0}}}{4} \epsilon_{1}^{3} \epsilon_{n 0}+\frac{2^{-f_{0}}}{2} \frac{2^{-f_{1}}}{2} \epsilon_{n 0} \epsilon_{n 1} \\
& \hat{z}_{3}=\hat{c}_{3}+\hat{n}_{2}=0.25-0.25 \epsilon_{0}^{3}-0.25 \epsilon_{1}^{3}-\frac{2^{-f_{0}}}{4} \epsilon_{n 0}-\frac{2^{-f_{1}}}{4} \epsilon_{n 1}+\frac{2^{-f_{2}}}{2} \epsilon_{n 2}+ \\
& +0.25 \epsilon_{0}^{3} \epsilon_{1}^{3}+\frac{2^{-f_{1}}}{4} \epsilon_{0}^{3} \epsilon_{n 1}+\frac{2^{-f_{0}}}{4} \epsilon_{1}^{3} \epsilon_{n 0}+\frac{2^{-f_{0}}}{2} \frac{2^{-f_{1}}}{2} \epsilon_{n 0} \epsilon_{n 1}, \\
& \hat{a}_{4}=\hat{x}_{4}+\hat{n}_{0}=0.5+0.5 \epsilon_{0}^{4}+\frac{2^{-f_{0}}}{2} \epsilon_{n 0}, \\
& \hat{b}_{4}=\hat{y}_{4}+\hat{n}_{1}=-0.5+0.5 \epsilon_{1}^{4}+\frac{2^{-f_{1}}}{2} \epsilon_{n 1}, \\
& \hat{c}_{4}=\hat{a}_{4} \hat{b}_{4}=-0.25-0.25 \epsilon_{0}^{4}+0.25 \epsilon_{1}^{4}-\frac{2^{-f_{0}}}{4} \epsilon_{n 0}+\frac{2^{-f_{1}}}{4} \epsilon_{n 1}+0.25 \epsilon_{0}^{4} \epsilon_{1}^{4}+ \\
& +\frac{2^{-f_{1}}}{4} \epsilon_{0}^{4} \epsilon_{n 1}+\frac{2^{-f_{0}}}{4} \epsilon_{1}^{4} \epsilon_{n 0}+\frac{2^{-f_{0}}}{2} \frac{2^{-f_{1}}}{2} \epsilon_{n 0} \epsilon_{n 1} \\
& \hat{d}_{4}=\hat{c}_{4}+\hat{n}_{2}=-0.25-0.25 \epsilon_{0}^{4}+0.25 \epsilon_{1}^{4}-\frac{2^{-f_{0}}}{4} \epsilon_{n 0}+\frac{2^{-f_{1}}}{4} \epsilon_{n 1}+\frac{2^{-f_{2}}}{2} \epsilon_{n 2}+ \\
& +0.25 \epsilon_{0}^{4} \epsilon_{1}^{4}+\frac{2^{-f_{1}}}{4} \epsilon_{0}^{4} \epsilon_{n 1}+\frac{2^{-f_{0}}}{4} \epsilon_{1}^{4} \epsilon_{n 0}+\frac{2^{-f_{0}}}{2} \frac{2^{-f_{1}}}{2} \epsilon_{n 0} \epsilon_{n 1}, \\
& \hat{z}_{4}=-\hat{d}_{4}=0.25+0.25 \epsilon_{0}^{4}-0.25 \epsilon_{1}^{4}+\frac{2^{-f_{0}}}{4} \epsilon_{n 0}-\frac{2^{-f_{1}}}{4} \epsilon_{n 1}-\frac{2^{-f_{2}}}{2} \epsilon_{n 2}- \\
& -0.25 \epsilon_{0}^{1} \epsilon_{1}^{4}-\frac{2^{-f_{1}}}{4} \epsilon_{0}^{4} \epsilon_{n 1}-\frac{2^{-f_{0}}}{4} \epsilon_{1}^{4} \epsilon_{n 0}-\frac{2^{-f_{0}}}{2} \frac{2^{-f_{1}}}{2} \epsilon_{n 0} \epsilon_{n 1} .
\end{aligned}
$$

Once we have calculated the expansions, we use the equations in Sections 3.2.4 and 3.3.3 to obtain the RON. For this example we set all $f_{w q i}, i=0,1,2$, to 8 bits. By only considering the noise terms (i.e. those that contain at least one $\epsilon_{i}$ ) when we apply Equation 3.32, we obtain an output noise variance of $2.1193 \cdot 10^{-6}$. The reference noise variance, calculated using Monte-Carlo simulations (with $10^{7}$ samples) is $2.1198 \cdot 10^{-6}$. We also calculate the SQNR of the system, which is $43.6034 \mathrm{~dB}$ for the reference MonteCarlo simulation and $43.6044 \mathrm{~dB}$ for our proposed methodology.

\subsection{Clustered noise injection}

Analytical models that use polynomial expansions with independent RVs to estimate the round-off noise of fixed-point implementations do not scale well, and the methods we presented in Sections 3.2 and 3.3 are not free from that drawback. Including the noise sources in the system makes the number of terms in the base to grow quickly and, although the theory behind it remains valid, the mathematical expressions become too large to be handled in reasonable times by numerical engines. Figure 3.12 shows how the number of terms in a PCE basis grows exponentially. 


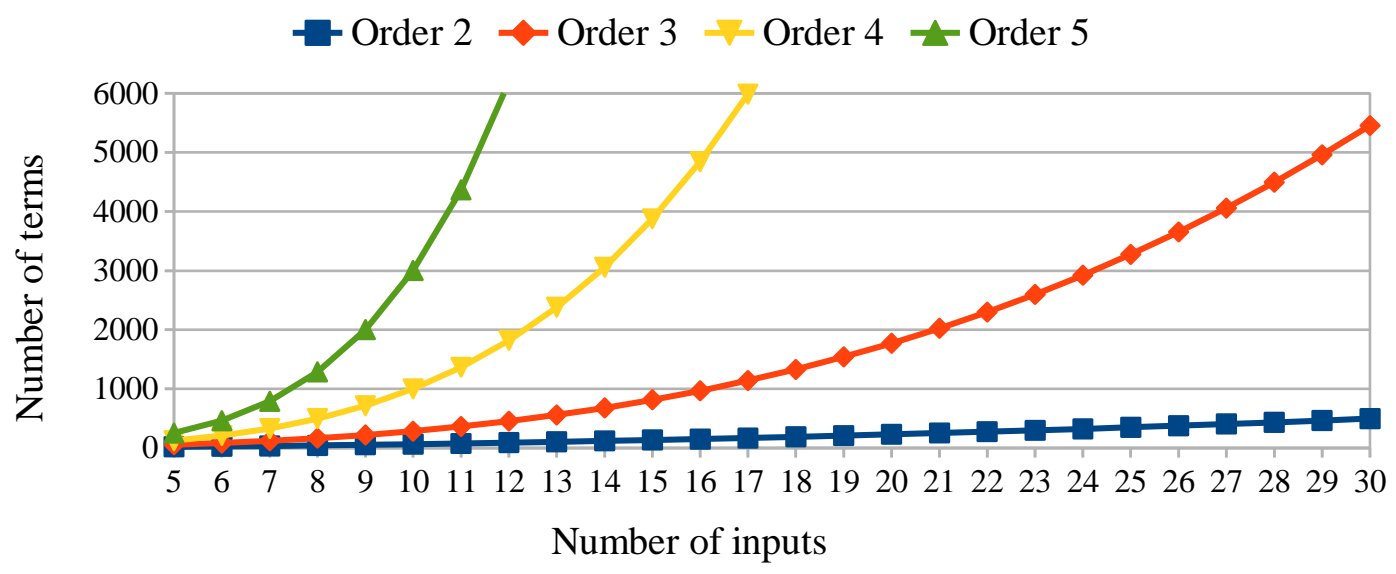

Fig. 3.12: Number of terms in a PCE base function of the order and number of RVs.

In this section, we present an iterative method based on a grouping technique that injects noise sources in the design in small groups that do not overload the numerical engines [SML14]. The propagation of these subgroups is studied independently and the results are combined at the end of the process. Although we apply the method to PCE and ME-gPC models, the proposed approach could be easily extended to other analytical frameworks. The rest of the section is organized as follows. Subsection 3.4.1 details the proposed methodology and Subsection 3.4.2 introduces a partitioning algorithm to select the most adequate way of grouping noise sources together.

\subsubsection{Methodology}

Figure 3.13 summarizes the proposed procedure to allow models based on polynomial expansions to introduce a large number of noise sources in a scalable way. First, we group all the noise sources we intend to introduce in the system in $N$ clusters with $N \leq N_{n s}$, where $N_{n s}$ is the total number of noise sources. In practice, a cluster must contain at least two noise sources so their interaction is properly modelled. Then, we process each cluster independently, injecting its noise sources to the system and propagating the coefficients.

Each of the resulting systems of equations will contain the information on how the corresponding cluster of noises distorts the signals, but will also include the coefficients representing clean signal values. As we are only interested in noise coefficients, we remove the clean signal coefficients from each system of equations. We do this simply subtracting the coefficient propagation through a clean system from each of the systems with noises. If the dynamic range was estimated using the same analytical principles, the signal coefficients are available at this stage, so the process is almost immediate.

The final solution for the noisy signal is obtained as the addition of every partial result alongside with the clean signal values. It retains all the information about how each individual noise interacts with the signals, how noises grouped together interact with each other and how those noises combined interact with signals. The idea behind 


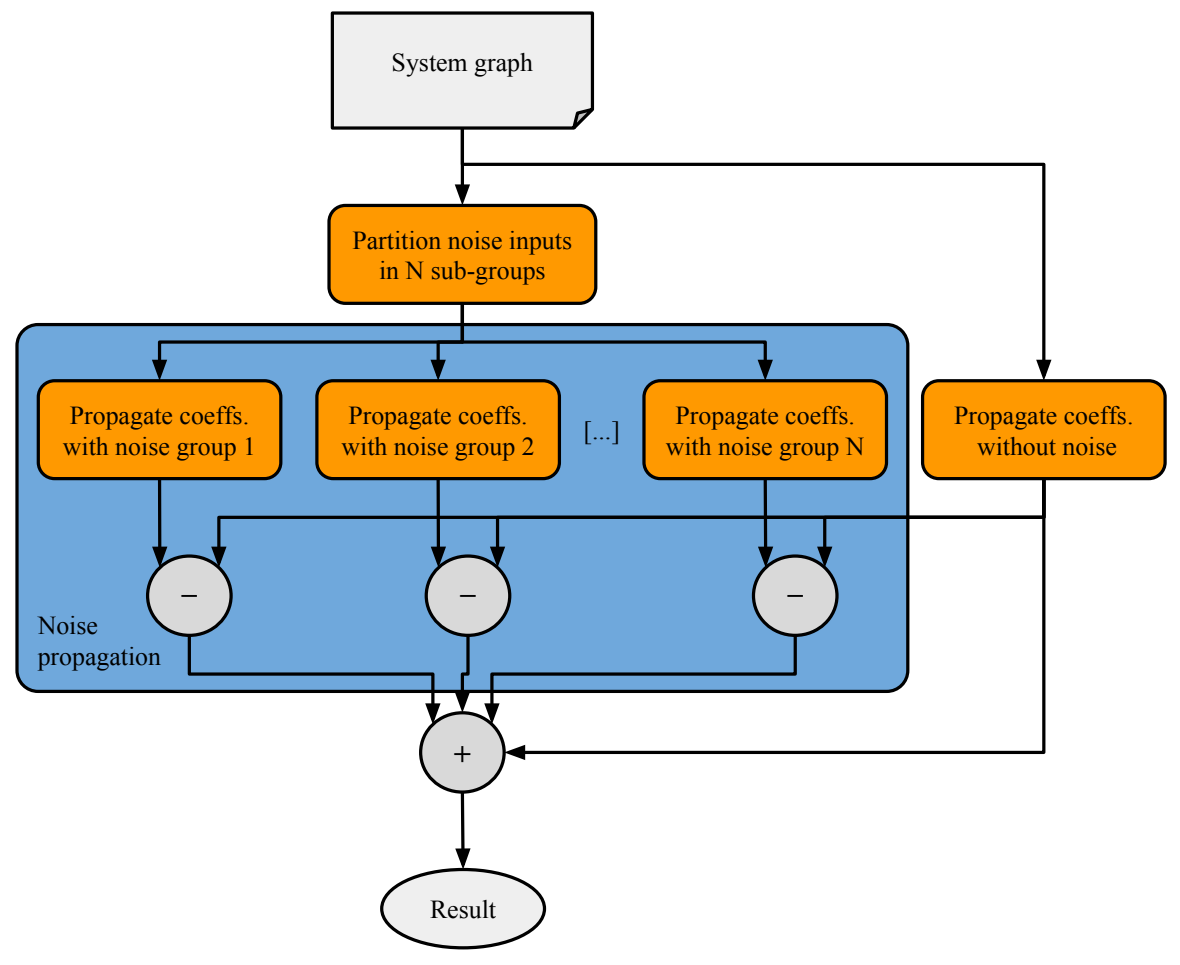

Fig. 3.13: Iterative noise injection algorithm flow.

this method is that the contribution of terms that are missing from the solution with respect to a system where all noises are injected at once will be small enough to be negligible. To model the RON we use Additive White Noise (AWN) sources following the DNM as we have shown in Equation 3.39. This model imposes that the magnitude of the noise must be much smaller than that of the signals. Since the missing terms will always be the result of signals multiplied by two or more noises, the contribution of the signal should be big enough to make up for the effect of the several noise contributions combined or the noise values should violate the DNM for the discarded contribution to be significant. The former, even though it may occur, is very unlikely to happen.

It must be noted that the selection of the clusters of noise sources may impact the accuracy of the results obtained with this method. In essence, it is best that noise sources that interact together are grouped together. We discuss this in the next subsection, and then we present a partitioning algorithm aimed at minimizing the loss of accuracy due to unsuitable grouping.

\subsubsection{Grouping of noise sources and impact on accuracy}

How noise sources are grouped together influences the accuracy of the solutions. To illustrate this, we will consider the example of the simple chain of operations depicted in Figure 3.14. The right side of the figure represents the system on the left after adding noise sources n1-n4.

We consider the following values for the input signals: 


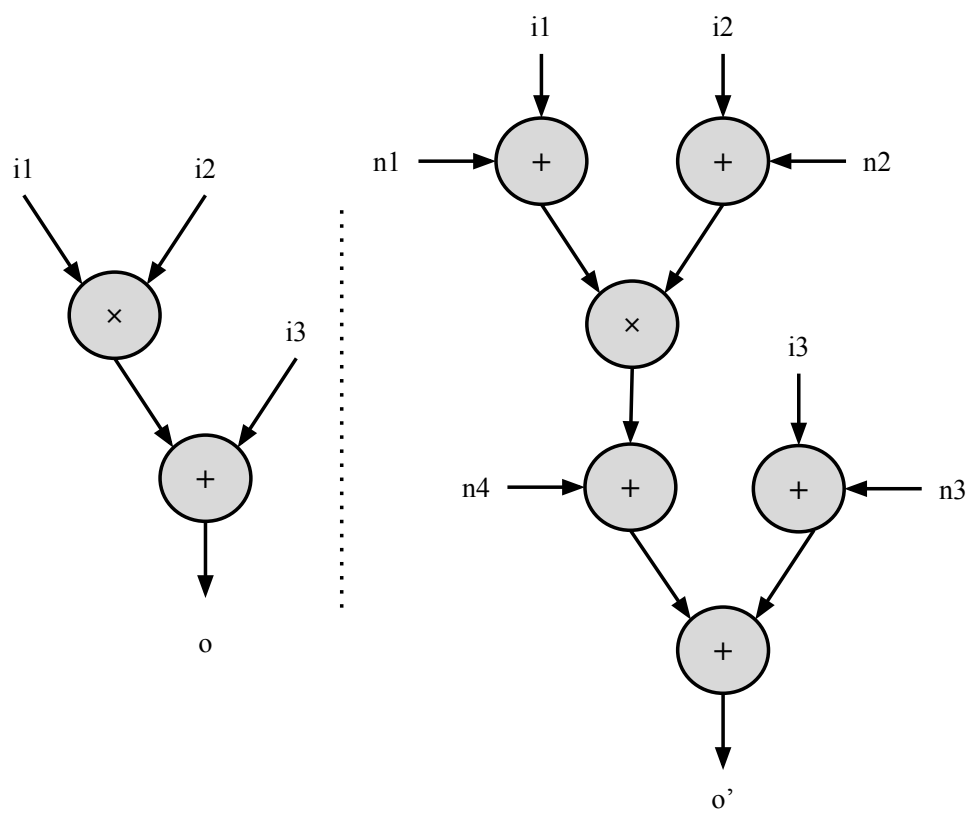

Fig. 3.14: Simple system without and with noise sources.

$$
\hat{i}[1-3]=i_{[1-3]},
$$

and, for the noise sources:

$$
\hat{n}[1-4]=\frac{2^{-7}}{2} \epsilon_{n[1-4]} .
$$

According to the rules presented in Section 3.1.3, and setting a maximum polynomial order of two, the propagation of the coefficients generates the following polynomials at the output:

$$
\hat{o^{\prime}}=\epsilon_{1} \epsilon_{2}+\epsilon_{3}+\frac{2^{-7}}{2} \epsilon_{1} \epsilon_{n 2}+\frac{2^{-7}}{2} \epsilon_{2} \epsilon_{n 1}+\frac{2^{-14}}{4} \epsilon_{n 1} \epsilon_{n 2}+\frac{2^{-7}}{2} \epsilon_{n 3}+\frac{2^{-7}}{2} \epsilon_{n 4} .
$$

If we apply the iterative noise injection methodology using sub-groups of two noises each, a possible grouping could be: $g_{1}=\left(n_{1}, n_{3}\right), g_{2}=\left(n_{2}, n_{4}\right)$, in which case the output value would be:

$$
\begin{gathered}
\hat{o}_{a 1}^{\hat{\prime}}=\epsilon_{1} \epsilon_{2}+\epsilon_{3}+\frac{2^{-7}}{2} \epsilon_{2} \epsilon_{n 1}+\frac{2^{-7}}{2} \epsilon_{n 3}, \\
\hat{o}_{a 2}^{\prime}=\epsilon_{1} \epsilon_{2}+\epsilon_{3}+\frac{2^{-7}}{2} \epsilon_{1} \epsilon_{n 2}+\frac{2^{-7}}{2} \epsilon_{n 4}, \\
\hat{o_{a}^{\prime}}=\epsilon_{1} \epsilon_{2}+\epsilon_{3}+\frac{2^{-7}}{2} \epsilon_{1} \mu_{2}+\frac{2^{-7}}{2} \epsilon_{2} \epsilon_{n 1}+\frac{2^{-7}}{2} \epsilon_{n 3}+\frac{2^{-7}}{2} \epsilon_{n 4},
\end{gathered}
$$

which presents some deviation from the result in Equation 3.51. But instead, if the 
grouping is $g_{1}=\left(n_{1}, n_{2}\right), g_{2}=\left(n_{3}, n_{4}\right)$, the resulting polynomials are:

$$
\begin{gathered}
\hat{o}_{b 1}^{\prime}=\epsilon_{1} \epsilon_{2}+\epsilon_{3}+\frac{2^{-7}}{2} \epsilon_{1} \epsilon_{n 2}+\frac{2^{-7}}{2} \epsilon_{2} \epsilon_{n 1}+\frac{2^{-14}}{4} \epsilon_{n 1} \epsilon_{n 2}, \\
\hat{o_{b 2}^{\prime}}=\epsilon_{1} \epsilon_{2}+\epsilon_{3}+\frac{2^{-7}}{2} \epsilon_{n 3}+\frac{2^{-7}}{2} \epsilon_{n 4}, \\
\hat{o_{b}^{\prime}}=\epsilon_{1} \epsilon_{2}+\epsilon_{3}+\frac{2^{-7}}{2} \epsilon_{1} \epsilon_{n 2}+\frac{2^{-7}}{2} \epsilon_{2} \epsilon_{n 1}+\frac{2^{-14}}{4} \epsilon_{n 1} \epsilon_{n 2}+\frac{2^{-7}}{2} \epsilon_{n 3}+\frac{2^{-7}}{2} \epsilon_{n 4}=\hat{o^{\prime}} .
\end{gathered}
$$

We can see that, in the second case, the output polynomial is identical to the one obtained with all the noise sources considered at once. Even though obtaining identical results when the size of the design grows may not always be possible to achieve, it is clear that convenient selection of groups can minimize the accuracy degradation of the output values.

\subsubsection{Hierarchical partitioning}

In order to constrain the degradation of the output estimations due to partitioning, we propose a fast partitioning algorithm based on a classical move-based iterative algorithm. It is possible to find recent and advanced partitioning methods based on genetic algorithms but since the fixed-point modelling and optimization problems themselves are already very time-consuming tasks, it is imperative to minimize the time dedicated to additional tasks. On this behalf, move-based iterative algorithms offer reasonably good results in minimal times. The interested reader may find exhaustive surveys on graph partitioning methods in [AK95] and [KHKM11].

\section{The Fiduccia and Mattheyses algorithm}

The Fiduccia and Mattheyses (FM) algorithm [FM82] proposes a bisection heuristic where a series of passes are iteratively performed. In each pass all movements are considered, even those with negative gains. This allows the algorithm to escape local minima. One of the main advantages of the FM method is that its execution time is linear with respect to the number of nodes in the graph. This is mainly achieved due to the use of the bucket list, a specialized data structure with constant access time to the node with the highest gain and fast update time for the gains after each move. Additional information about the highest gain non-empty bucket is also held in the bucket list, so the access time is linear. Also, the number of elements to have in each partition is relaxed by introducing a balance condition that allows a certain degree of variation between the partition sizes. During the execution passes only those moves that do not violate the balance condition are allowed. Nowadays this algorithm is considered a reference for comparison purposes when a new partitioning heuristic is proposed. 


\section{Problem definition}

The input for the partitioning algorithm is a Data Flow Graph (DFG), with a set $I=\left\{i_{1}, i_{2}, \cdots, i_{n}\right\}$ of independent inputs that relate among them through $F=$ $\left\{f_{1}, f_{2}, \cdots, f_{j}\right\}$ operations to generate $O=\left\{o_{1}, o_{2}, \cdots, o_{k}\right\}$ outputs. The information is propagated from the inputs to the outputs as defined by the set $E=\left\{e_{1}, e_{2}, \cdots, e_{m}\right\}$ of directed edges, where $e_{p} \in(I, F) \cup(F, F) \cup(F, O) \cup(I, O)$. To simplify the formulation of the problem, we define the DFG following the notation in [AK95] as $G=(V, E)$ where $V=I \cup F \cup O$.

We consider the decomposition of a given DFG as valid if it satisfies the following requisites:

- The number of noise sources in the final subsystems is balanced (within a degree of variation).

- The number of noise sources in each of the final subsystems is less than a certain established value.

To calculate the number of noise sources in each partition, we will assume that every $e_{q} \in E$ will be quantized and, thus, a noise source will be injected in it. Also, due to the characteristics of this problem, for each cut we will generate a new input in the sub-graph that includes the destination node of the cut edge.

We formalize the problem as follows:

Given a graph $G=(V, E)$ with weighted nodes and two parameters $s$ (partition size) and $b$ (balance condition),

Find a multi-level, hierarchical partitioning of $G$ where $G^{p-1}=\bigcup_{i=0}^{q} G_{i}^{p}$ and $\forall i, j$, $i \neq j, G_{i}^{p} \cap G_{j}^{p}=\emptyset$, being $G^{n}$ a sub-graph of level $n$ and $G_{m}$ the $m$-th partition of a graph. For each partition, the difference in number of noise sources per sub-graph with less than or equal to $s$ inputs must be less than $b$ and, for each level, the number of edges crossing between partitions (the cut set size, $C_{s}$ ) must be minimized.

Figure 3.15 shows an example of a valid decomposition. On the left side we present the original DFG . Dark grey nodes with an arrow are inputs or outputs, depending on the direction of the arrow. The nodes in white are operators. On the right side we show a decomposition of the DFG where we assume that each partition can contain a maximum of $4( \pm 1)$ inputs. Dark grey nodes with dotted lines are new inputs generated during the partitioning process, while the light grey nodes indicate the source of the cut edges. The relationships between sources and new inputs are indicated in the orange box at the bottom.

\section{Partitioning algorithm}

To hierarchically decompose a DFG, we adapt the FM algorithm to our grouping method. It is iteratively applied to each of the subsystems in the partition hierarchy that do not comply with the stop criterion to generate a new partition level. 

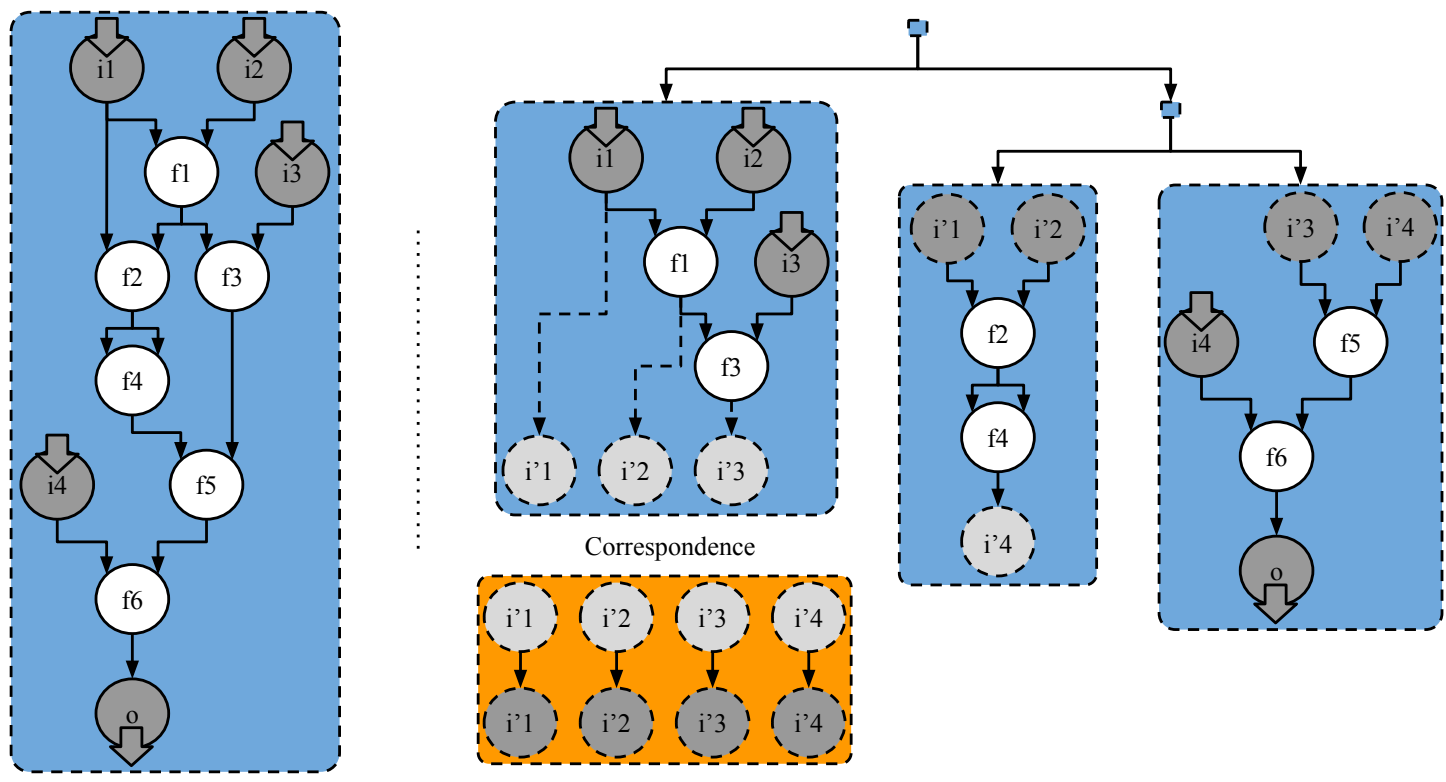

Fig. 3.15: Example of the hierarchical decomposition of a DFG.

One of the main problems of the FM algorithm is that the quality of the solution strongly depends on the initial partition, which is generated randomly. We overcome this issue by running several passes of the FM partitioning, each of them with a different initial state, and using only the best (i.e. the one that minimizes the cost function) of the obtained final partitions. Given the fast execution time of the FM algorithm, we can do this a number of times $\left(N_{\text {test }}\right)$ without incurring into excessive overall execution times. We present the pseudocode for the complete partitioner in Algorithm 2.

DFG representation It is common to use hypergraphs [Ber76] when solving partitioning problems with the FM algorithm. Assuming that an hyperedge includes all the edges that connect a node with its successors, we define a Single Source Directed Hypergraph (SSD Hypergraph). Each hyperedge is represented as a list of the nodes in the structure and an indicator of which of them is the source. We then redefine the graph as $\hat{G}=(V, \hat{E})$, where $\hat{E}=\left(\hat{e}_{1}, \hat{e}_{2}, \cdots, \hat{e}_{k}\right)$. Each $\hat{e}_{i}=\left(v_{s}, L_{d}\right)$ being $L_{d}$ the list of all $v_{d}$ that verify $\left(v_{s}, v_{d}\right) \in E$. If the list is empty no hyperedge is generated (i.e. there are no hyperedges with elements from $O$ as source).

This representation allows us to identify the direction of the edges with minimal memory occupation. To detect whether an hyperedge belongs to a given cutset we check if any of the nodes from the list is not in the same partition as the source. Since cutting an edge generates new nodes in the graph, the SSD Hypergraph notation permits us to identify quickly if a node has to be added to a partition simply by checking if the source belongs to it or not.

Cost function The objective of the partitioning algorithm is to obtain a well-balanced number of noise sources in each partition while minimizing $C_{s}$. To this end, we count 


\section{CHAPTER 3. QUANTIZATION OF SYSTEMS WITH CONTROL FLOW STRUCTURES}

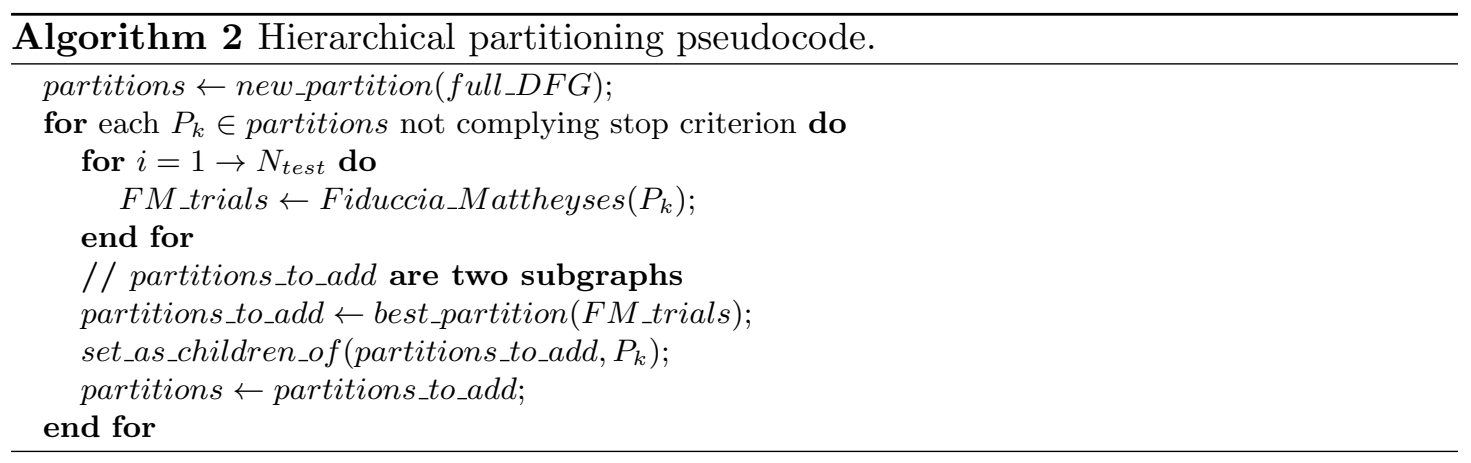

the number of edges in each partition.

It must be taken into account that we have to include new inputs to the partitions when an hyperedge is cut, so the corresponding noise source can be added. The partition that does not contain the source node of the cut hyperedge gets one more input. Thus, the resulting subsystems will have different number of inputs depending on the final cutset.

Considering all of the above, the cost function for a given partition $G_{m}^{p}$ is the number of edges in the sub-graph plus the number of cut SSD Hyperedges that incide in the partition. The expression of the cost is

$$
f_{\text {cost }}\left(G_{m}^{p}\right)=\left|E_{m}\right|+\left|\hat{e}_{i} \rightarrow \hat{e}_{i} \in \operatorname{cs}\left(G^{p-1}\right) \wedge \operatorname{src}\left(\hat{e}_{i}\right) \notin V_{m}^{p}\right|,
$$

being $E_{m}$ the edges in the partition, $V_{m}^{p}$ the list of nodes of $G_{m}^{p}, \operatorname{cs}\left(G^{p-1}\right)$ the list of hyperedges cut in the partition of the parent level of $G_{m}^{p}$ and $\operatorname{src}\left(\hat{e}_{i}\right)$ the source node of the hypernode $\hat{e}_{i}$.

Balance and stop criteria The balance criterion we consider differs from the one proposed in [FM82]. In our case, the allowed deviation is set as a function of the total number of inputs of the graph while keeping the largest cell size as its lower bound.

The stop criterion depends exclusively on the computational capabilities of the system in which we will run the algorithms as well as on how efficient and optimized are the analytical model and the numerical engine. These parameters may impact the number of uncertainties (i.e. inputs of the DFG) that can be handled in reasonable times by the numerical engines. Thus, we set the iterative partitioning to stop when the number of uncertainties in the partitions reaches the limit that the analytical model can handle.

In Chapter 4 we show a case study of the application of the clustered noise injection method to accelerate the modelling process and we show how to combine it with the modelling of systems with control-flow structures in a single, unified process. 


\subsection{Conclusions}

Analytical models for the study of the dynamic range and the RON in fixed-point systems have evolved over the years to broaden their scope of applicability. Also, they have moved towards automated procedures capable of handling the system descriptions and the propagation rules with less and less interaction from the designers. Among these analytical models, those based on extensions of AA have proven to achieve very accurate results in short computation times. In particular, the combination of MAA with PCE has allowed to model high order correlations between signals in systems with non-linear operators, which in turn has been used to model the RON of this type of systems. But this approach is not capable of handling control-flow structures, which in practice constrains its applicability to very basic systems.

In this chapter we have extended the MAA/PCE model to model systems that contain control-flow structures. We have adapted parts of the general ME-gPC methodology, which naturally extends PCE, to study systems that contain two general types of controlflow structures: choice operators (if ...then... else) and loop structures (while...do). We have presented an homogeneous and comprehensive methodology that allows us to automatically generate accurate estimates of the dynamic ranges and the RON in systems with such operators. Also, we have illustrated its usage through a simple example. This approach is capable of delivering the same accurate results as MAA/PCE while encompassing a whole new range of systems that could not be studied before through extensions of AA.

Given the inherent complexity of non-linear systems and the need to track the correlation between signals, most analytical approaches that aim at modelling this type of systems suffer from scalability issues. The methodologies based on extensions of AA are not an exception to this rule, and specially with MAA/PCE the number of terms in a polynomial can grow exponentially. In this chapter we have also addressed this issue, introducing a strategy for clustered noise injection, where we effectively control the number of RON sources that are introduced in the system at every step of the process. We have also proposed an iterative partitioning technique based on the classical FM algorithm to minimize the possible loss of accuracy due to the clustering. This approach automatically adapts to the structure of the targeted design and searches for the grouping that minimizes the connections between partitions.

The methods we have presented in this chapter have an additional advantage with respect to most analytical models to date. All the techniques we propose rely at some point on dividing the system and studying each partition independently from the rest, only combining the results at the end of the process. This offers a very high degree of parallelism, and thus the methods can benefit greatly from high-end hardware platforms such as GPUs and computing clusters.

Furthermore, in Chapter 4 we present several case studies to illustrate the use of the methodologies we have proposed in this chapter. 


\section{Chapter 4}

\section{System modelling case studies}

The descent to Hell is easy; the black gate stands open, night and day; but to climb back again, to retrace one's steps to the upper air, there is the hard task, the toil.

Virgil, Aeneid (Book VI)

In Chapter 3 we have described a new methodology based on the ideas from ME-gPC and we have combined them with MAA/PCE to model the dynamic range and evaluate the round-off noise of fixed-point architectures with control-flow operations. Also, we have introduced a scalable methodology that inserts the RON noise sources in clustered groups to keep the sizes of the polynomials at manageable levels. In this chapter we apply these techniques step by step to several case studies and we present the obtained results to prove the validity and accuracy of our approaches.

All the case studies use rounding quantizers (see Section 3.3.1). The experiments are run on a single-threaded code running on an computer equipped with an Intel ${ }^{\circledR}$

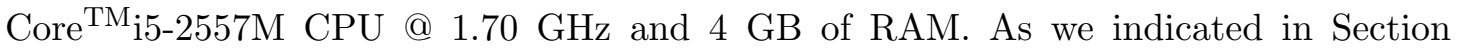
3.5, execution times could be shortened by parallelizing the code. Also, as indicated in Section 3.3, we do not consider the effects of the RON in the outcome of the decision operations.

We present different scenarios and we show how the proposed methodology provides accurate results for all of them. First, in Section 4.1 we apply MAA/PCE to a real life case study to prove its validity. Section 4.2 shows the modelling of dynamic range and RON of a non-linear system with a choice operator. Section 4.3 presents a case study with a loop structure, and in Section 4.4 we apply our methodology to a system with nested control-flow operations. The case study in Section 4.5 applies the clustered noise injection technique to a DFG without control-flow structures. Finally, we combine the dynamic range and the RON modelling with the clustered noise injection in Section 4.6. 


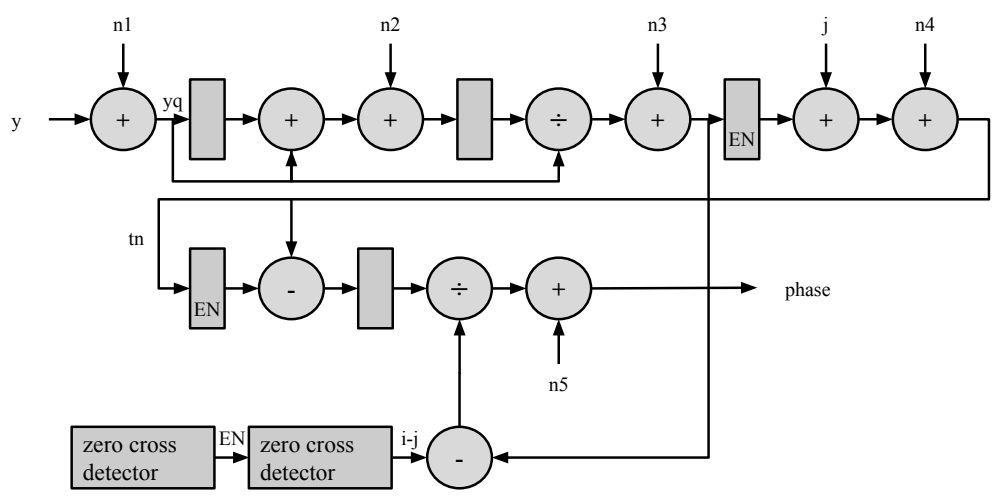

Fig. 4.1: Phase measuring stage of the TJ-II interpolation algorithm with quantization inputs.

Table 4.1: SQNR obtained for different fractional word-lengths after quantizing the whole interferometer case study.

\begin{tabular}{|c|c|}
\hline Bits & SQNR \\
\hline \hline 5 & 30.1358 \\
\hline 6 & 36.1531 \\
\hline 7 & 42.1737 \\
\hline 8 & 48.1943 \\
\hline 9 & 54.2149 \\
\hline 10 & 60.2355 \\
\hline 11 & 66.2561 \\
\hline 12 & 72.2767 \\
\hline 13 & 78.2973 \\
\hline 14 & 84.3179 \\
\hline 15 & 90.3385 \\
\hline 16 & 96.3591 \\
\hline
\end{tabular}

\subsection{Initial study: Interferometer}

As part of the work for this Ph.D. Thesis we have studied the viability of the MAA/PCE approach to a real life problem. For this, we have studied the FPGA-based processing design currently used in the TJ-II infrared interferometer to compute the electron density $\left[\mathrm{ELS}^{+} 13\right]$. This work was done as part of a project along with the Centro de Investigaciones Energéticas, Medioambientales y Tecnológicas (CIEMAT), Madrid. The system we analyse in this case study is shown in Figure 4.1.

Following the methodology described in Section 3.1.3 we quantize the system to several uniform word-lengths. The different sources of error present in the interferometer are analysed in [SEKH08] and we set a limit of $62 \mathrm{~dB}$ to the maximum SQNR allowed. Table 4.1 shows this SQNR is satisfied using a quantization of all the signals and fractional units to 11 fractional bits. 

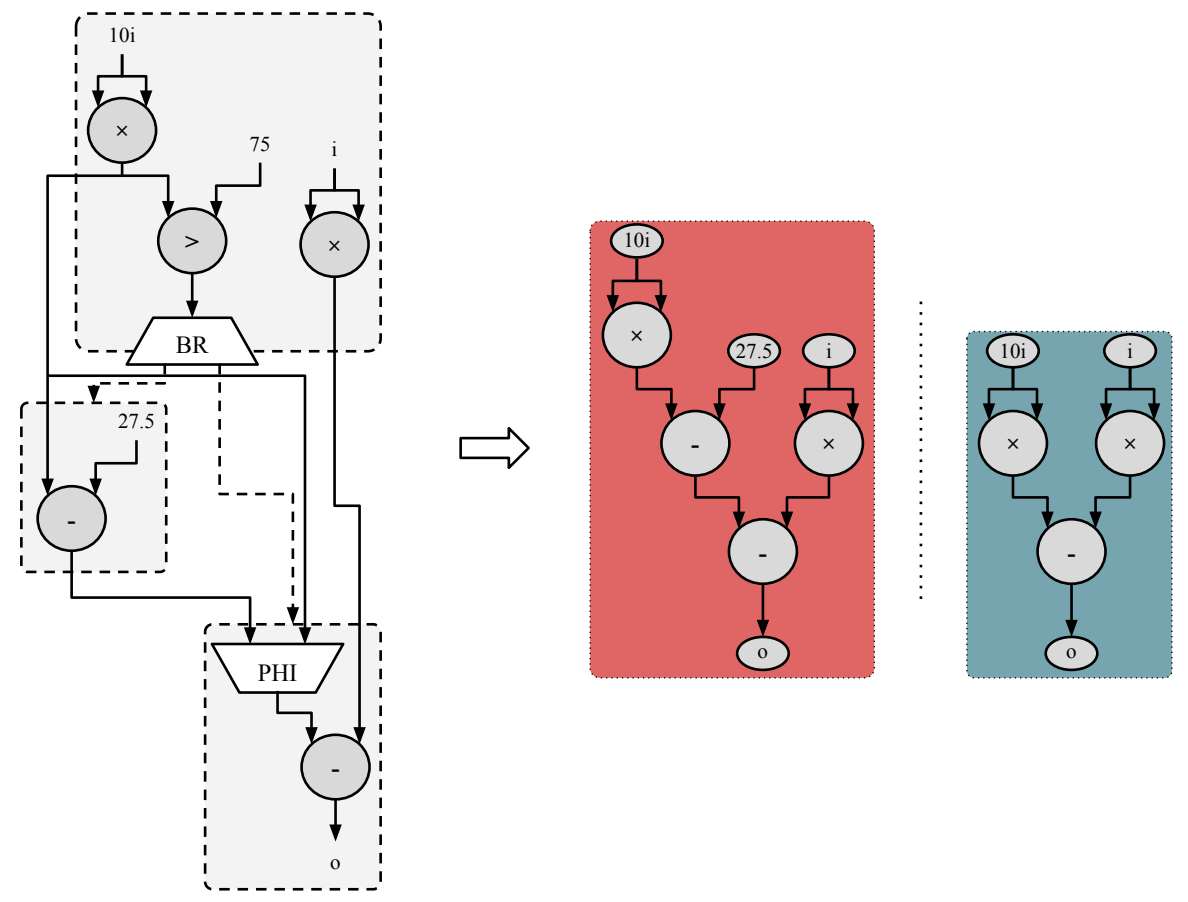

Fig. 4.2: Basic Blocks diagram and derivative DFGs of the Teager case study.

\subsection{Choice operators: Teager+}

In this case study we show how the methodology we propose is capable of representing non-linear systems accurately. To that end, we study the system in Figure 4.2 (left). It is based on an example extracted from the Teager algorithm [MS00]. The original system has been used in different works [WZN04b, WZN06, Est11] to show how PCE achieves better results than other techniques in the presence of non-linearities. We introduce a choice operator to it in which the left-hand side of the subtraction is modified if the value is larger than a certain quantity. For this case study we consider i $U[-1,1]$.

\subsubsection{Teager+: Dynamic range estimation}

In order to estimate the dynamic range of the system, we first need to generate the derivative DFGs and map the different sub-domains to them as explained in Section 3.2. In this case there is only one control-flow structure, a choice operator, to treat. By considering the paths on both sides of the decision separately we obtain the DFGs shown in Figure 4.2 (right).

To solve the conditional, we propagate the PCE coefficients from the input of the system to the input of the choice operator

$$
\begin{gathered}
\hat{i}=\epsilon, \\
10 \hat{i}=10 \epsilon, \\
\hat{a}=10 \hat{i} * 10 \hat{i}=\frac{100}{3}+\frac{200}{3}\left(\frac{1}{2}\left(3 \epsilon^{2}-1\right)\right) .
\end{gathered}
$$




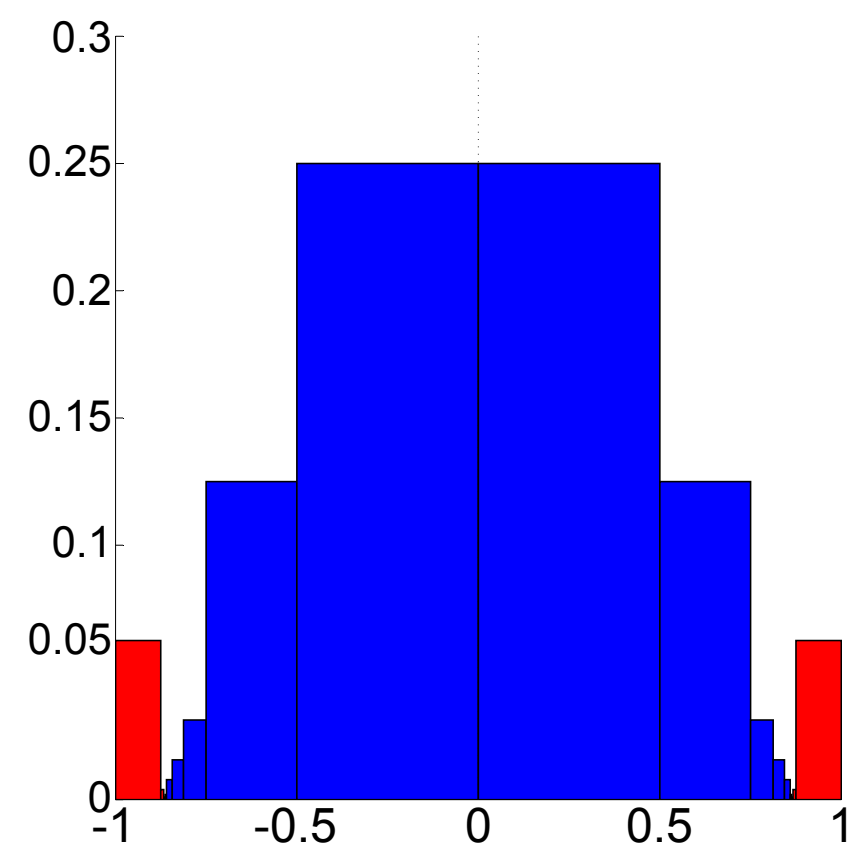

Fig. 4.3: Partitions per decision output direction in the Teager case study.

Thus, the inequation we have to solve in order to determine the outcome of the choice operator is:

$$
\frac{100}{3}+\frac{200}{3}\left(\frac{1}{2}\left(3 \epsilon^{2}-1\right)\right)>75
$$

Partitioning the input domain iteratively as we explained in Section 3.2 we determine which regions of the input domain are mapped to each derivative DFG. In this case study, the polynomial base has three elements: the independent term (1) and both first $(\mu)$ and second order $\left(\frac{1}{2}\left(3 \epsilon^{2}-1\right)\right)$ terms. As we indicated in Section 3.2.2, each time we study a sub-domain to determine if it needs further partitioning we choose $k=10\left(N_{p}+1\right)$ random points in the uniformly distributed domain. Consequently, for this test case we set $k=30$.

Depending on the value the designer chooses for $J_{l i m}$, the number of partitions will vary while the methodology tries to match the exact frontier where the decision output changes direction. Figure 4.3 shows the 28 domain partitions we obtain with $J_{l i m}=10^{-4}$. The horizontal axis represents the input domain $d[-1,1]$ for $\epsilon$, while the vertical axis represents the associated $J_{k}$ for each partition. The colour of each bar matches the background of the DFG in Figure 4.2 to which the partition corresponds.

The propagation of the PCE coefficients through each derivative DFG is as follows, where subscript $r$ indicates the propagation through the DFG in Figure 4.2 in the red background (left), and subscript $b$ indicates the propagation through the DFG in the 
blue background (right):

$$
\begin{gathered}
\hat{i}_{r}=i_{0, r}+i_{1, r} \epsilon, \\
10 \hat{i}_{r}=10 i_{0, r}+10 i_{1, r} \epsilon, \\
\hat{a}_{r}=10 \hat{i}_{r} * 10 \hat{i}_{r}=100 i_{0, r}^{2}+\frac{100 i_{1, r}^{2}}{3}+200 i_{0, r} i_{1, r} \epsilon+\frac{200 i_{1, r}^{2}}{3}\left(\frac{1}{2}\left(3 \epsilon^{2}-1\right)\right), \\
\hat{b}_{r}=\hat{i}_{r} * \hat{i}_{r}=i_{0, r}^{2}+\frac{i_{1, r}^{2}}{3}+2 i_{0, r} i_{1, r} \epsilon+\frac{2 i_{1, r}^{2}}{3}\left(\frac{1}{2}\left(3 \epsilon^{2}-1\right)\right), \\
\hat{c}_{r}=\hat{a}_{r}-27.5=100 i_{0, r}^{2}+\frac{100}{3} i_{1, r}^{2}-27.5+200 i_{0, r} i_{1, r} \epsilon+\frac{200}{3} i_{1, r}^{2}\left(\frac{1}{2}\left(3 \epsilon^{2}-1\right)\right), \\
\hat{o}_{r}=\hat{c}_{r}-\hat{b}_{r}=99 i_{0, r}^{2}+33 i_{1, r}^{2}-27.5+198 i_{0, r} i_{1, r} \epsilon+66 i_{1, r}^{2}\left(\frac{1}{2}\left(3 \epsilon^{2}-1\right)\right), \\
\hat{a}_{b}=10 \hat{i}_{b}=10 i_{0, b}+10 i_{1, b} \epsilon, \\
\hat{b}_{b}=\hat{i}_{b} * \hat{i}_{b}=100 i_{0, b}^{2}+\frac{100 i_{1, b}^{2}}{3}+200 i_{0, b} i_{1, b} \epsilon+\frac{200 i_{1, b}^{2}}{3}\left(\frac{1}{2}\left(3 \epsilon^{2}-1\right)\right), \\
\hat{o}_{b, b}^{2}+\frac{i_{1, b}^{2}}{3}+2 i_{0, b} i_{1, b} \epsilon+\frac{2 i_{1, b}^{2}}{3}\left(\frac{1}{2}\left(3 \epsilon^{2}-1\right)\right), \\
\hat{a}_{b}-\hat{b}_{b}=99 i_{0, b}^{2}+33 i_{1, b}^{2}+198 i_{0, b} i_{1, b} \epsilon+66 i_{1, b}^{2}\left(\frac{1}{2}\left(3 \epsilon^{2}-1\right)\right) .
\end{gathered}
$$

The coefficients for each of the 28 partitions we obtain with $J_{\text {lim }}=10^{-4}$ are listed in Table 4.2. Replacing the coefficients in the corresponding set of equations we obtain the results for each sub-domain. Then, we apply the equations in Section 3.2.4 to obtain the combined statistical moments of the system. We use a Monte-Carlo simulation of $10^{7}$ samples as reference, which gives an output mean value 29.3210 and a variance value 565.9719. Applying the coefficients in Table 4.2 to Equations 4.1 and 4.2 we compute a mean value 29.3141 and a variance 565.9894 . These results deviate $0.02 \%$ from both reference values. Table 4.3 summarizes the results we obtain for different values of $J_{\text {lim }}$, including the number of partitions generated, the execution times and the error percentage with respect to the reference for both mean and variance values.

\subsubsection{Teager+: RON modelling}

After modelling the dynamic range of the system, we compute the RON using the approach we have described in Section 3.3. First, we add noise sources to each operation in the derivative subsystems, assigning them carefully so the fractional word-lengths match corresponding nodes in each DFG, as we explained in Section 3.3.1. In this case study we need a noise source for the input and four for the operations. We name these sources $\hat{e}_{i}, i=1,2,3,4,5$. Using the DNM we determine their values for a quantization of 8 fractional bits: 
Table 4.2: PCE coefficients for Teager + with $J_{\text {lim }}=10^{-4}$.

\begin{tabular}{|c|c|c|c|c|c|}
\hline Partition & $\alpha_{0, r}$ & $\alpha_{1, r}$ & $\alpha_{0, b}$ & $\alpha_{1, r}$ & $J_{k}$ \\
\hline 1 & -0.9375 & 0.0625 & - & - & 0.0625 \\
\hline 2 & -0.8711 & 0.0039 & - & - & 0.0039 \\
\hline 3 & -0.8667 & 0.0005 & - & - & 0.0005 \\
\hline 4 & -0.8661 & 0.0001 & - & - & 0.0001 \\
\hline 5 & - & - & -0.8660 & 0.0001 & 0.0001 \\
\hline 6 & - & - & -0.8658 & 0.0001 & 0.0001 \\
\hline 7 & - & - & -0.8655 & 0.0002 & 0.0002 \\
\hline 8 & - & - & -0.8643 & 0.0010 & 0.0010 \\
\hline 9 & - & - & -0.8613 & 0.0020 & 0.0020 \\
\hline 10 & - & - & -0.8516 & 0.0078 & 0.0078 \\
\hline 11 & - & - & -0.8281 & 0.0156 & 0.0156 \\
\hline 12 & - & - & -0.7813 & 0.0312 & 0.0313 \\
\hline 13 & - & - & -0.6250 & 0.1250 & 0.1250 \\
\hline 14 & - & - & -0.2500 & 0.2500 & 0.2500 \\
\hline 15 & - & - & 0.2500 & 0.2500 & 0.2500 \\
\hline 16 & - & - & 0.6250 & 0.1250 & 0.1250 \\
\hline 17 & - & - & 0.7813 & 0.0313 & 0.0313 \\
\hline 18 & - & - & 0.8281 & 0.0156 & 0.0156 \\
\hline 19 & - & - & 0.8516 & 0.0078 & 0.0078 \\
\hline 20 & - & - & 0.8613 & 0.0020 & 0.0020 \\
\hline 21 & - & - & 0.8643 & 0.0010 & 0.0010 \\
\hline 22 & - & - & 0.8655 & 0.0002 & 0.0002 \\
\hline 23 & - & - & 0.8658 & 0.0001 & 0.0001 \\
\hline 24 & 0.8660 & 0.0001 & - & - & 0.0001 \\
\hline 25 & 0.8661 & 0.0001 & - & - & 0.0001 \\
\hline 26 & 0.8667 & 0.0005 & - & - & 0.0005 \\
\hline 27 & 0.8711 & 0.0039 & - & - & 0.0039 \\
\hline 28 & 0.9375 & 0.0625 & - & - & 0.0625 \\
\hline
\end{tabular}

Table 4.3: System statistics for different values of $J_{\text {lim }}$ in the Teager+ case study.

\begin{tabular}{|c|c|c||c|c||c|c|}
\hline$J_{\text {lim }}$ & Partitions & Exec. Time & Mean & $\%$ error & Variance & $\%$ error \\
\hline \hline $10^{-2}$ & 14 & $12 \mathrm{~s}$ & 29.5625 & $0.82 \%$ & 581.6980 & $2.79 \%$ \\
\hline $10^{-3}$ & 20 & $27 \mathrm{~s}$ & 29.2402 & $0.27 \%$ & 561.3084 & $0.8 \%$ \\
\hline $10^{-4}$ & 28 & $44 \mathrm{~s}$ & 29.3141 & $0.02 \%$ & 565.9894 & $0.02 \%$ \\
\hline $10^{-5}$ & 34 & $1 \mathrm{~m} 15 \mathrm{~s}$ & 29.3149 & $0.02 \%$ & 565.9370 & $0.01 \%$ \\
\hline
\end{tabular}



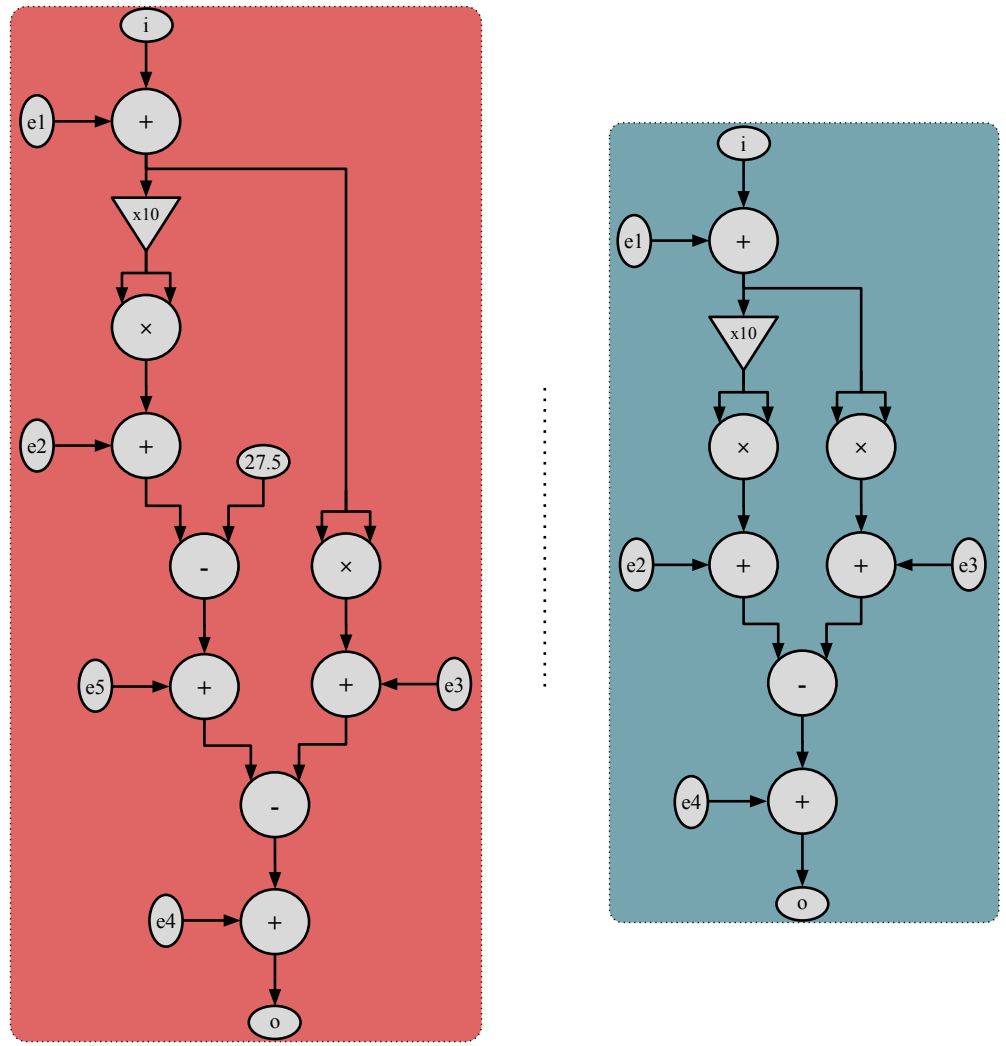

Fig. 4.4: Derivarive DFGs from Figure 4.2 including the noise sources.

$$
\begin{gathered}
\hat{e}_{1}=\frac{2^{-8}}{2} \epsilon_{1}, \\
\hat{e}_{2}=\left(\frac{2^{-8}}{2}+\frac{2^{-15}}{2}\right) \epsilon_{2} \approx \frac{2^{-8}}{2} \epsilon_{2}, \\
\hat{e}_{3}=\left(\frac{2^{-8}}{2}+\frac{2^{-15}}{2}\right) \epsilon_{3} \approx \frac{2^{-8}}{2} \epsilon_{3}, \\
\hat{e}_{4}=\hat{e}_{5}=0 .
\end{gathered}
$$

We modify the derivative sub-systems from Figure 4.2 to include the corresponding noise sources, obtaining the DFGs in Figure 4.4.

We propagate the input coefficients as follows. Again, subscript $r$ indicates the propagation through the DFG in Figure 4.4 in the red background (left), while subscript $b$ indicates the propagation through the DFG in the blue background (right):

$$
\begin{gathered}
\hat{d}_{r}=\hat{i}_{r}+\hat{n}_{1}=\alpha_{0, r}+\alpha_{1, r} \mu+\frac{2^{-8}}{2} \epsilon_{1}, \\
10 \hat{d}_{r}=10 \alpha_{0, r}+10 \alpha_{1, r} \mu+10 \frac{2^{-8}}{2} \epsilon_{1}, \\
\hat{a}_{r}=10 \hat{d}_{r} * 10 \hat{d}_{r}=100 \alpha_{0, r}^{2}+\frac{100}{3} \alpha_{1, r}^{2}+\frac{100}{3} \frac{2^{-16}}{4}+200 \alpha_{0, r} \alpha_{1, r} \mu+200 \frac{2^{-8}}{2} \alpha_{0, r} \epsilon_{1}+ \\
+\frac{200}{3} \alpha_{1, r}^{2}\left(\frac{1}{2}\left(3 \mu^{2}-1\right)\right)+200 \frac{2^{-8}}{2} \alpha_{1, r} \epsilon_{1} \mu+\frac{200}{3} \frac{2^{-16}}{4}\left(\frac{1}{2}\left(3 \epsilon_{1}^{2}-1\right)\right),
\end{gathered}
$$




$$
\begin{aligned}
& \hat{b}_{r}=\hat{d}_{r} * \hat{d}_{r}=\alpha_{0, r}^{2}+\frac{1}{3} \alpha_{1, r}^{2}+\frac{2^{-16}}{12}+2 \alpha_{0, r} \alpha_{1, r} \mu+2^{-8} \alpha_{0, r} \epsilon_{1}+ \\
& +\frac{2}{3} \alpha_{1, r}^{2}\left(\frac{1}{2}\left(3 \mu^{2}-1\right)\right)+2^{-8} \alpha_{1, r} \mu \epsilon_{1}+\frac{2^{-15}}{12}\left(\frac{1}{2}\left(3 \epsilon_{1}^{2}-1\right)\right) \\
& \hat{f}_{r}=\hat{a}_{r}+\hat{n}_{2}=100 \alpha_{0, r}^{2}+\frac{100}{3} \alpha_{1, r}^{2}+\frac{100}{3} \frac{2^{-16}}{4}+200 \alpha_{0, r} \alpha_{1, r} \mu+200 \frac{2^{-8}}{2} \alpha_{0, r} \epsilon_{1}+ \\
& +\frac{2^{-8}}{2} \epsilon_{2}+\frac{200}{3} \alpha_{1, r}^{2}\left(\frac{1}{2}\left(3 \mu^{2}-1\right)\right)+200 \frac{2^{-8}}{2} \alpha_{1, r} \epsilon_{1} \mu+\frac{200}{3} \frac{2^{-16}}{4}\left(\frac{1}{2}\left(3 \epsilon_{1}^{2}-1\right)\right), \\
& \hat{g}_{r}=\hat{b}_{r}+\hat{n}_{3}=\alpha_{0, r}^{2}+\frac{1}{3} \alpha_{1, r}^{2}+\frac{2^{-16}}{12}+2 \alpha_{0, r} \alpha_{1, r} \mu+2^{-8} \alpha_{0, r} \epsilon_{1}+\frac{2^{-8}}{2} \epsilon_{3}+ \\
& +\frac{2}{3} \alpha_{1, r}^{2}\left(\frac{1}{2}\left(3 \mu^{2}-1\right)\right)+2^{-8} \alpha_{1, r} \mu \epsilon_{1}+\frac{2^{-15}}{12}\left(\frac{1}{2}\left(3 \epsilon_{1}^{2}-1\right)\right) \\
& \hat{c}_{r}=\hat{f}_{r}-27.5=100 \alpha_{0, r}^{2}+\frac{100}{3} \alpha_{1, r}^{2}-27.5+\frac{100}{3} \frac{2^{-16}}{4}+200 \alpha_{0, r} \alpha_{1, r} \mu+200 \frac{2^{-8}}{2} \alpha_{0, r} \epsilon_{1}+ \\
& +\frac{2^{-8}}{2} \epsilon_{2}+\frac{200}{3} \alpha_{1, r}^{2}\left(\frac{1}{2}\left(3 \mu^{2}-1\right)\right)+200 \frac{2^{-8}}{2} \alpha_{1, r} \epsilon_{1} \mu+\frac{200}{3} \frac{2^{-16}}{4}\left(\frac{1}{2}\left(3 \epsilon_{1}^{2}-1\right)\right), \\
& \hat{k}_{r}=\hat{c}_{r}+\hat{n}_{5}=100 \alpha_{0, r}^{2}+\frac{100}{3} \alpha_{1, r}^{2}-27.5+\frac{100}{3} \frac{2^{-16}}{4}+200 \alpha_{0, r} \alpha_{1, r} \mu+200 \frac{2^{-8}}{2} \alpha_{0, r} \epsilon_{1}+ \\
& +\frac{2^{-8}}{2} \epsilon_{2}+\frac{200}{3} \alpha_{1, r}^{2}\left(\frac{1}{2}\left(3 \mu^{2}-1\right)\right)+200 \frac{2^{-8}}{2} \alpha_{1, r} \epsilon_{1} \mu+\frac{200}{3} \frac{2^{-16}}{4}\left(\frac{1}{2}\left(3 \epsilon_{1}^{2}-1\right)\right), \\
& \hat{h}_{r}=\hat{k}_{r}-\hat{g}_{r}=99 \alpha_{0, r}^{2}+33 \alpha_{1, r}^{2}+33 \frac{2^{-16}}{4}-27.5+198 \alpha_{0, r} \alpha_{1, r} \mu+ \\
& +99 \cdot 2^{-8} \alpha_{0, r} \epsilon_{1}+\frac{2^{-8}}{2} \epsilon_{2}-\frac{2^{-8}}{2} \epsilon_{3}+66 \alpha_{1, r}^{2}\left(\frac{1}{2}\left(3 \mu^{2}-1\right)\right)+ \\
& +99 \cdot 2^{-8} \alpha_{1, r} \mu \epsilon_{1}+66 \frac{2^{-16}}{4}\left(\frac{1}{2}\left(3 \epsilon_{1}^{2}-1\right)\right), \\
& \hat{o}_{r}=\hat{h}_{r}+\hat{n}_{4}=99 \alpha_{0, r}^{2}+33 \alpha_{1, r}^{2}+33 \frac{2^{-16}}{4}-27.5+198 \alpha_{0, r} \alpha_{1, r} \mu+ \\
& +99 \cdot 2^{-10} \alpha_{0, r} \epsilon_{1}+\frac{2^{-8}}{2} \epsilon_{2}-\frac{2^{-8}}{2} \epsilon_{3}+ \\
& +66 \alpha_{1, r}^{2}\left(\frac{1}{2}\left(3 \mu^{2}-1\right)\right)+99 \cdot 2^{-8} \alpha_{1, r} \mu \epsilon_{1}+66 \frac{2^{-16}}{4}\left(\frac{1}{2}\left(3 \epsilon_{1}^{2}-1\right)\right) \\
& \begin{array}{c}
\hat{d}_{b}=\hat{i}_{b}+\hat{n}_{1}=\alpha_{0, b}+\alpha_{1, b} \mu+\frac{2^{-8}}{2} \epsilon_{1}, \\
10 \hat{d}_{b}=10 \alpha_{0, b}+10 \alpha_{1, b} \mu+10 \frac{2^{-8}}{2} \epsilon_{1},
\end{array} \\
& \hat{a}_{b}=10 \hat{d}_{b} * 10 \hat{d}_{b}=100 \alpha_{0, b}^{2}+\frac{100}{3} \alpha_{1, b}^{2}+\frac{100}{3} \frac{2^{-16}}{4}+200 \alpha_{0, b} \alpha_{1, b} \mu+200 \frac{2^{-8}}{2} \alpha_{0, b} \epsilon_{1}+ \\
& +\frac{200}{3} \alpha_{1, b}^{2}\left(\frac{1}{2}\left(3 \mu^{2}-1\right)\right)+200 \frac{2^{-8}}{2} \alpha_{1, b} \epsilon_{1} \mu+\frac{200}{3} \frac{2^{-16}}{4}\left(\frac{1}{2}\left(3 \epsilon_{1}^{2}-1\right)\right), \\
& \hat{b}_{b}=\hat{d}_{b} * \hat{d}_{b}=\alpha_{0, b}^{2}+\frac{1}{3} \alpha_{1, b}^{2}+\frac{2^{-16}}{12}+2 \alpha_{0, b} \alpha_{1, b} \mu+2^{-10} \alpha_{0, b} \epsilon_{1}+ \\
& +\frac{2}{3} \alpha_{1, b}^{2}\left(\frac{1}{2}\left(3 \mu^{2}-1\right)\right)+2^{-8} \alpha_{1, b} \mu \epsilon_{1}+\frac{2^{-15}}{12}\left(\frac{1}{2}\left(3 \epsilon_{1}^{2}-1\right)\right) \\
& \hat{f}_{b}=\hat{a}_{b}+\hat{n}_{2}=100 \alpha_{0, b}^{2}+\frac{100}{3} \alpha_{1, b}^{2}+\frac{100}{3} \frac{2^{-16}}{4}+200 \alpha_{0, b} \alpha_{1, b} \mu+200 \frac{2^{-8}}{2} \alpha_{0, b} \epsilon_{1}+ \\
& +\frac{2^{-8}}{2} \epsilon_{2}+\frac{200}{3} \alpha_{1, b}^{2}\left(\frac{1}{2}\left(3 \mu^{2}-1\right)\right)+200 \frac{2^{-8}}{2} \alpha_{1, b} \epsilon_{1} \mu+\frac{200}{3} \frac{2^{-16}}{4}\left(\frac{1}{2}\left(3 \epsilon_{1}^{2}-1\right)\right), \\
& \hat{g}_{b}=\hat{b}_{b}+\hat{n}_{3}=\alpha_{0, b}^{2}+\frac{1}{3} \alpha_{1, b}^{2}+\frac{2^{-16}}{12}+2 \alpha_{0, b} \alpha_{1, b} \mu+2^{-8} \alpha_{0, b} \epsilon_{1}+\frac{2^{-8}}{2} \epsilon_{3}+ \\
& +\frac{2}{3} \alpha_{1, b}^{2}\left(\frac{1}{2}\left(3 \mu^{2}-1\right)\right)+2^{-8} \alpha_{1, b} \mu \epsilon_{1}+\frac{2^{-15}}{12}\left(\frac{1}{2}\left(3 \epsilon_{1}^{2}-1\right)\right)
\end{aligned}
$$




$$
\begin{aligned}
\hat{h}_{b}=\hat{f}_{b}-\hat{g}_{b}=99 \alpha_{0, b}^{2}+33 \alpha_{1, b}^{2}+33 \frac{2^{-18}}{4}+198 \alpha_{0, b} \alpha_{1, b} \mu+99 \cdot 2^{-10} \alpha_{0, b} \epsilon_{1}+\frac{2^{-8}}{2} \epsilon_{2}- \\
\\
-\frac{2^{-8}}{2} \epsilon_{3}+66 \alpha_{1, b}^{2}\left(\frac{1}{2}\left(3 \mu^{2}-1\right)\right)+99 \cdot 2^{-8} \alpha_{1, b} \mu \epsilon_{1}+66 \frac{2^{-18}}{4}\left(\frac{1}{2}\left(3 \epsilon_{1}^{2}-1\right)\right), \\
\hat{o}_{b}=\hat{h}_{b}+\hat{n}_{4}=99 \alpha_{0, b}^{2}+33 \alpha_{1, b}^{2}+33 \frac{2^{-18}}{4}+198 \alpha_{0, b} \alpha_{1, b} \mu+99 \cdot 2^{-8} \alpha_{0, b} \epsilon_{1}+\frac{2^{-8}}{2} \epsilon_{2}- \\
\\
-\frac{2^{-8}}{2} \epsilon_{3}+66 \alpha_{1, b}^{2}\left(\frac{1}{2}\left(3 \mu^{2}-1\right)\right)+99 \cdot 2^{-8} \alpha_{1, b} \mu \epsilon_{1}+66 \frac{2^{-16}}{4}\left(\frac{1}{2}\left(3 \epsilon_{1}^{2}-1\right)\right) .
\end{aligned}
$$

Then, we keep the noise terms only. This leaves us with the following:

$$
\begin{gathered}
n \hat{o}_{d}=100 \cdot 2^{-8} \alpha_{0, d} \epsilon_{1}+\frac{2^{-8}}{2} \epsilon_{2}-\frac{2^{-8}}{2} \epsilon_{3}+ \\
+99 \cdot 2^{-8} \alpha_{1, d} \mu \epsilon_{1}+66 \frac{2^{-16}}{4}\left(\frac{1}{2}\left(3 \epsilon_{1}^{2}-1\right)\right), \quad d=r, b .
\end{gathered}
$$

Applying first the coefficients in Table 4.2 to the corresponding PCE expansion and then the equations in Section 3.3.3 we obtain a variance 0.0170 , which in turn we use to obtain a SQNR value 45.2350. We use a Monte-Carlo simulation (with $10^{7}$ samples) as reference, which returns a variance 0.0166. With this, we compute a SQNR value 45.3269 , showing how our method is able to achieve very precise results in non-linear systems with control-flow structures. Also, the process takes only $2.6 \mathrm{~s}$.

\subsection{Loop structures: Accumulator}

To demonstrate how the modelling of loop structures is carried out we model the following function, with a $U[7,10]$ and $\mathrm{b} U[2,4]$ :

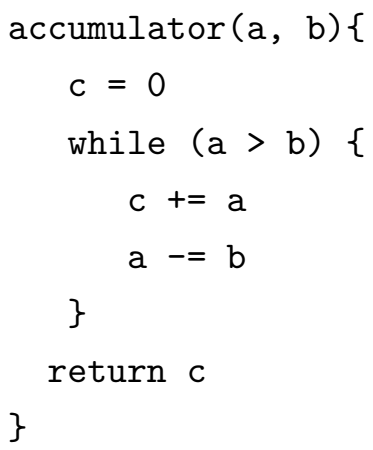

The code generates the system graph in Figure 4.5.

\subsubsection{Accumulator: Dynamic range estimation}

Applying the procedure described in Algorithm 1 (Section 3.2), we determine that the output of the conditional in Basic Block 2 is always going to be evaluated to True, so we only generate the derivative system graph for that case, which is equivalent to: 


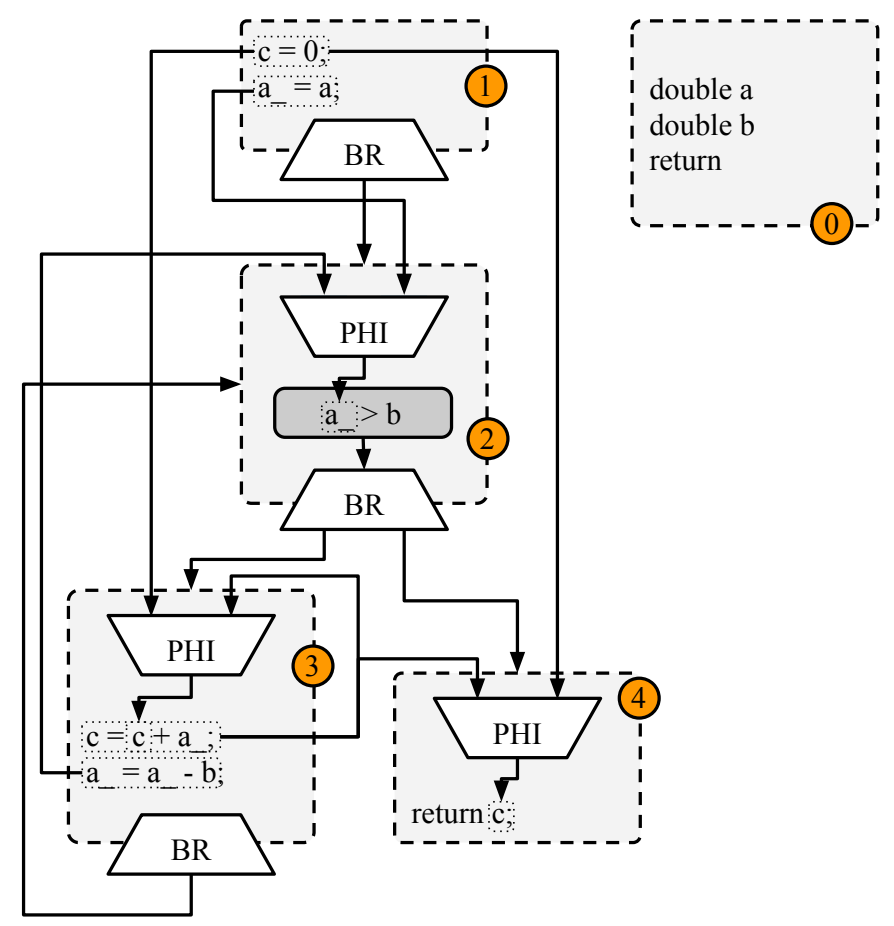

Fig. 4.5: Basic Blocks diagram of the loop case study.

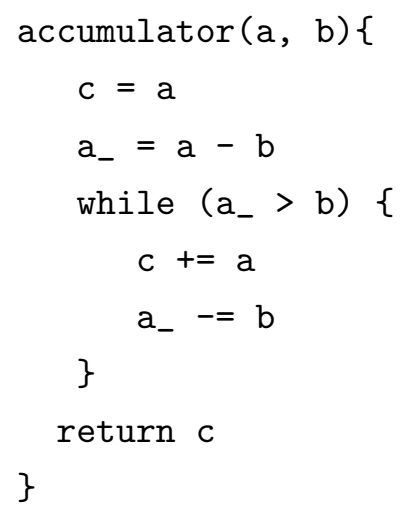

In the next iteration, the PCE expansion for the value of $\mathrm{a}_{-}$we use in the condition of the while loop is $\hat{a_{-}}=5.5+1.5 \epsilon_{a}-\epsilon_{b}$. This gives us the condition $5.5+1.5 \epsilon_{a}-\epsilon_{b}>3+\epsilon_{b}$, which is not always True. Consequently, the domain is partitioned. Depending on the value we determine for $J_{\text {lim }}$ we obtain a different number of partitions. At this point of the processing our procedure will have generated 121 partitions with $J_{\text {lim }}=10^{-3}$, a number that increases to 193 when $J_{\text {lim }}=10^{-4}$. We will use the latter value for the rest of the case study. As the input domain is directly related to the number of times a loop will iterate, designers will need to take this into consideration when choosing a value of $J_{l i m}$, as smaller values increase the domain partitioning time for each control-flow structure.

We have now, after the partitioning, two different system graphs. The first of them, in which the while is evaluated to False, has no more control flow structures to model. Figure 4.6 represents all the partitions of the domain, coloured according to last iteration 


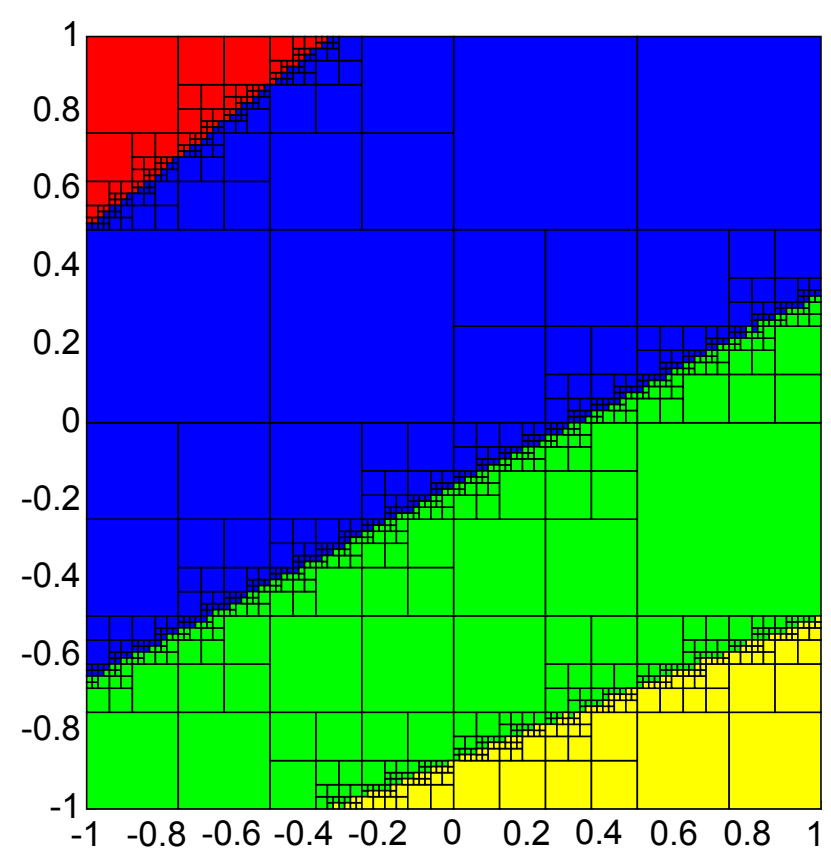

Fig. 4.6: Partitions per iteration of the loop case study.
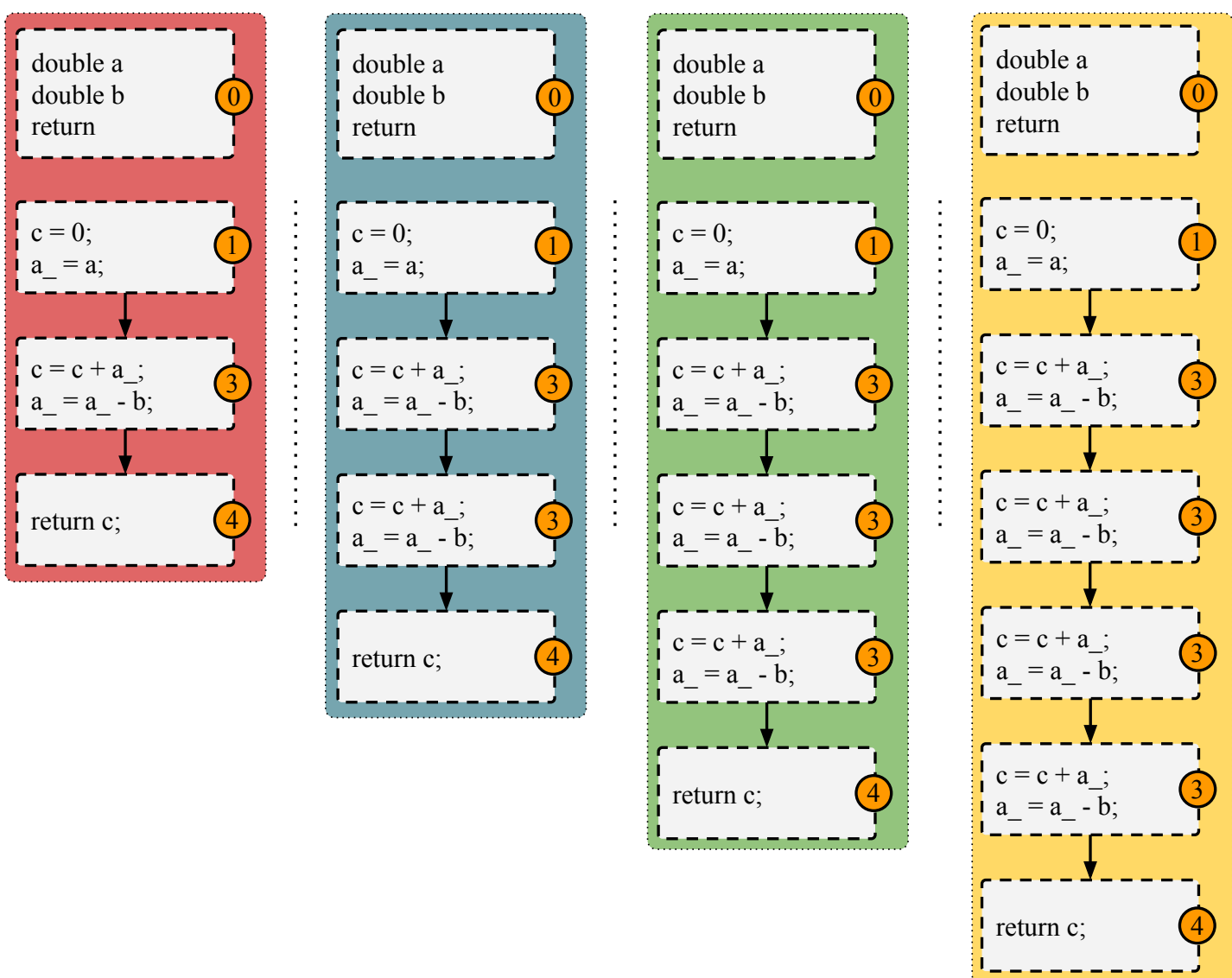

Fig. 4.7: Data Flow Graphs generated by the analysis of the loop case study. 
Table 4.4: System statistics for different values of $J_{l i m}$ in the Accumulator case study.

\begin{tabular}{|c|c|c||c|c||c|c|}
\hline$J_{\text {lim }}$ & Partitions & Exec. Time & Mean & $\%$ error & Variance & $\%$ error \\
\hline \hline $10^{-2}$ & 118 & $4 \mathrm{~m} 49 \mathrm{~s}$ & 15.6836 & $0.11 \%$ & 17.2355 & $0.74 \%$ \\
\hline $10^{-3}$ & 250 & $11 \mathrm{~m}$ & 15.7043 & $0.02 \%$ & 17.0490 & $0.34 \%$ \\
\hline $10^{-4}$ & 1069 & $59 \mathrm{~m} 47 \mathrm{~s}$ & 15.7035 & $0.01 \%$ & 17.1148 & $0.04 \%$ \\
\hline $10^{-5}$ & 4330 & $5 \mathrm{~h} 57 \mathrm{~m} 46 \mathrm{~s}$ & 15.7014 & $0.002 \%$ & 17.1092 & $0.01 \%$ \\
\hline
\end{tabular}

of the loop. The partitions that are mapped to this first system graph are coloured in red. The system graph generated by following the True branch still has a control flow operation, so part of the aforementioned 193 partitions still need further processing and partitioning.

Once the loop has been unfolded one more iteration, an additional partitioning is performed in the corresponding domain partitions. This reveals the need of yet another unroll of the system graph. The partitions that correspond to the False branch at this stage are coloured in blue in Figure 4.6. Continuing the process with the following iteration, we still find several sub-domains where partitioning is required. The partitions mapped to the False branch in this iteration are coloured in green in Figure 4.6.

The analysis of the remaining system graph with control-flow structure shows that the condition of the while loop will not be evaluated to True any more, so that the branch is discarded. The remaining partitions are coloured in yellow in Figure 4.6. The generated system graphs and the group of partitions they are mapped to are represented in Figure 4.7. For this case study, our procedure generates a total of 1069 partitions.

Once the partitioning is completed, we propagate the coefficients of each partition through their corresponding system graphs. By doing this, and applying the pertinent equations, we obtain a mean value 15.7035 and a variance 17.1148. Compared to the results obtained from Monte-Carlo simulations $\left(N=10^{7}\right.$, mean 15.7018 and variance 17.1074), the values obtained by the proposed method show deviations of $0.01 \%$ and $0.04 \%$ respectively. Table 4.4 summarizes the results for different values of $J_{\text {lim }}$.

\subsubsection{Accumulator: RON modelling}

To model the RON in the system we proceed to generate the noise sources that we will add to each operation. For this case study we need 10 of them. We denote each of them as $\hat{e}_{v, b . i}$ where $v$ is the name of the variable, $b$ the Basic Block that contains the variable, and $i$ the number of unrolled loop iteration, if any. Following this naming convention, the noise sources are $\hat{e}_{a, 0}, \hat{e}_{b, 0}, \hat{e}_{c, 3.1}, \hat{e}_{a_{-}, 3.1}, \hat{e}_{c, 3.2}, \hat{e}_{a_{-}, 3.2}, \hat{e}_{c, 3.3}, \hat{e}_{a_{-}, 3.3}, \hat{e}_{c, 3.4}$ and $\hat{e}_{c, 4}$. Considering the discussion from Section 3.3.1 about finite word-length assignments, in this case study we only require $5 f_{w q}$ different values as we show in Table 4.5.

Following the procedure in Section 3.3, we modify the derivative subsystems to incorporate the noise sources and we propagate the PCE coefficients of the inputs of each 
Table 4.5: $f_{w q}$ assignment to each noise source in the Accumulator case study.

\begin{tabular}{|c|c|}
\hline$f_{w q}$ & Noise sources \\
\hline \hline$f_{a}$ & $\hat{e}_{a, 0}$ \\
\hline$f_{b}$ & $\hat{e}_{b, 0}$ \\
\hline$f_{c 1}$ & $\hat{e}_{c, 3.1}, \hat{e}_{c, 3.2}, \hat{e}_{c, 3.3}, \hat{e}_{c, 3.4}$ \\
\hline$f_{a_{-}}$ & $\hat{e}_{a-3.1}, \hat{e}_{a_{-}, 3.2}, \hat{e}_{a-3.3}$ \\
\hline$f_{c 2}$ & $\hat{e}_{c, 4}$ \\
\hline
\end{tabular}

domain partition through the corresponding subsystem. As a result we generate a total of 1069 equations. After propagating the coefficients, we apply the equations in Section 3.3.3 to compute the statistical moments for the noise in the system. Considering a uniform quantization to 8 fractional bits, our approach estimates a variance value $1.6789 \cdot 10^{-5}$, which is used to get a SQNR value 60.0836 . The Monte-Carlo simulation $\left(10^{7}\right.$ samples) we use as reference returns a variance value $1.6647 \cdot 10^{-5}$ which we use to calculate a SQNR 60.1205. The execution time is $2 \mathrm{~m} 10 \mathrm{~s}$.

\subsection{Nested control-flow structures: Selector}

As we stated before, the presented scheme is also valid when the system contains different nested control-flow structures. To demonstrate this, in this case study we model a multi-choice selection done by means of nested if..then..else statements with a while loop in one of its branches. This shows the behaviour of the methodology when several control-flow structures appear in cascade. The code for the selector is:

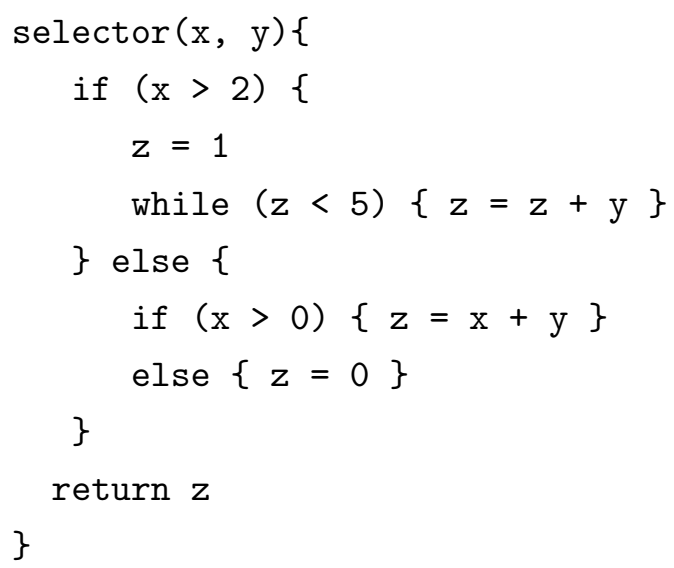

For this case, we assume $\mathrm{x} U[-1,6]$ and y $U[1.5,3]$ and we use a second order PCE. The input polynomials, thus, are $\hat{x}=2.5+3.5 \epsilon_{x}$ and $\hat{y}=2.25+0.75 \epsilon_{y}$.

\subsubsection{Selector: Dynamic range estimation}

Setting $J_{\text {lim }}=10^{-4}$, our methodology analyses the first conditional (if $(x>2)$ ) and splits the input domain $[-1,1]^{2}$ in 382 sub-domains divided in two groups. It generates the two derivative system graphs, assigns each sub-domain to the corresponding 


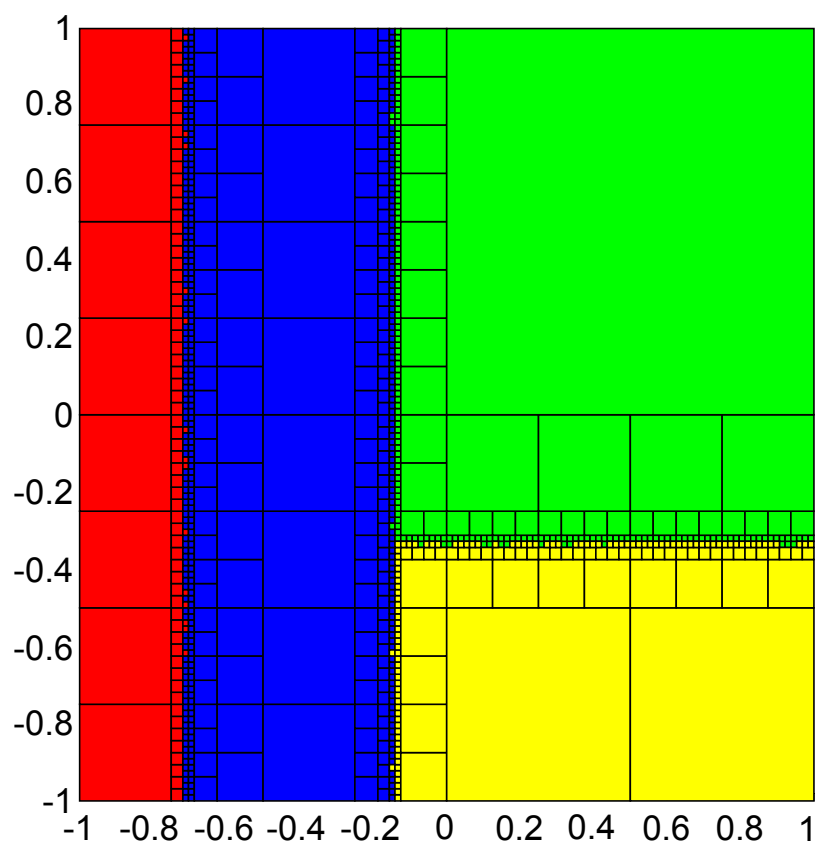

Fig. 4.8: Partitions per condition and iteration of the nested conditionals case study.

one, and continues the processing. In the case of the second conditional (i.e. the if ( $\mathrm{x}$ $>0$ ) nested under the else statement), the system graph is partitioned in two further derivative systems. The analysis of the branch containing the while loop determines that the first iteration of the loop will always be carried out, so the system graph is unrolled and the False branch of the condition discarded. Then, the second time the condition is analysed, we need to split some of the sub-domains to discern the direction of the decision. The third time the condition is checked, we see that all the sub-domains mapped to it resolve to False, so we generate the last derivative system graph and the partitioning stops. In total, the process generates 964 partitions. All this process is carried out automatically, without intervention from the user. The analytical study of the dynamic ranges of the signals and their propagation through the system graph allows the method to detect when a given branch of a loop structure will not be traversed any more.

Figure 4.8 shows how the domain is partitioned and how each sub-domain is mapped to a different derivative system graph. The partitions in red correspond to both if ( $\mathrm{x}$ $>2)$ and if $(x>0)$ conditions resolving to False. Partitions in blue correspond to if $(x>2)$ evaluated to False but if $(x>0)$ being True. Finally, the green and yellow sub-domains correspond to the two while loop unrolls, respectively. Figure 4.9 shows the execution paths corresponding to each group of partitions.

To compute the reference values for the system statistics we carry out Monte-Carlo simulations with $10^{7}$ samples and obtain a mean value 4.4038 and a variance 5.0845.

Propagating the input coefficients for each sub-domain and applying the equations in Section 3.2.4 we obtain a mean value 4.4064 and a variance 5.0682. Our methodology, 

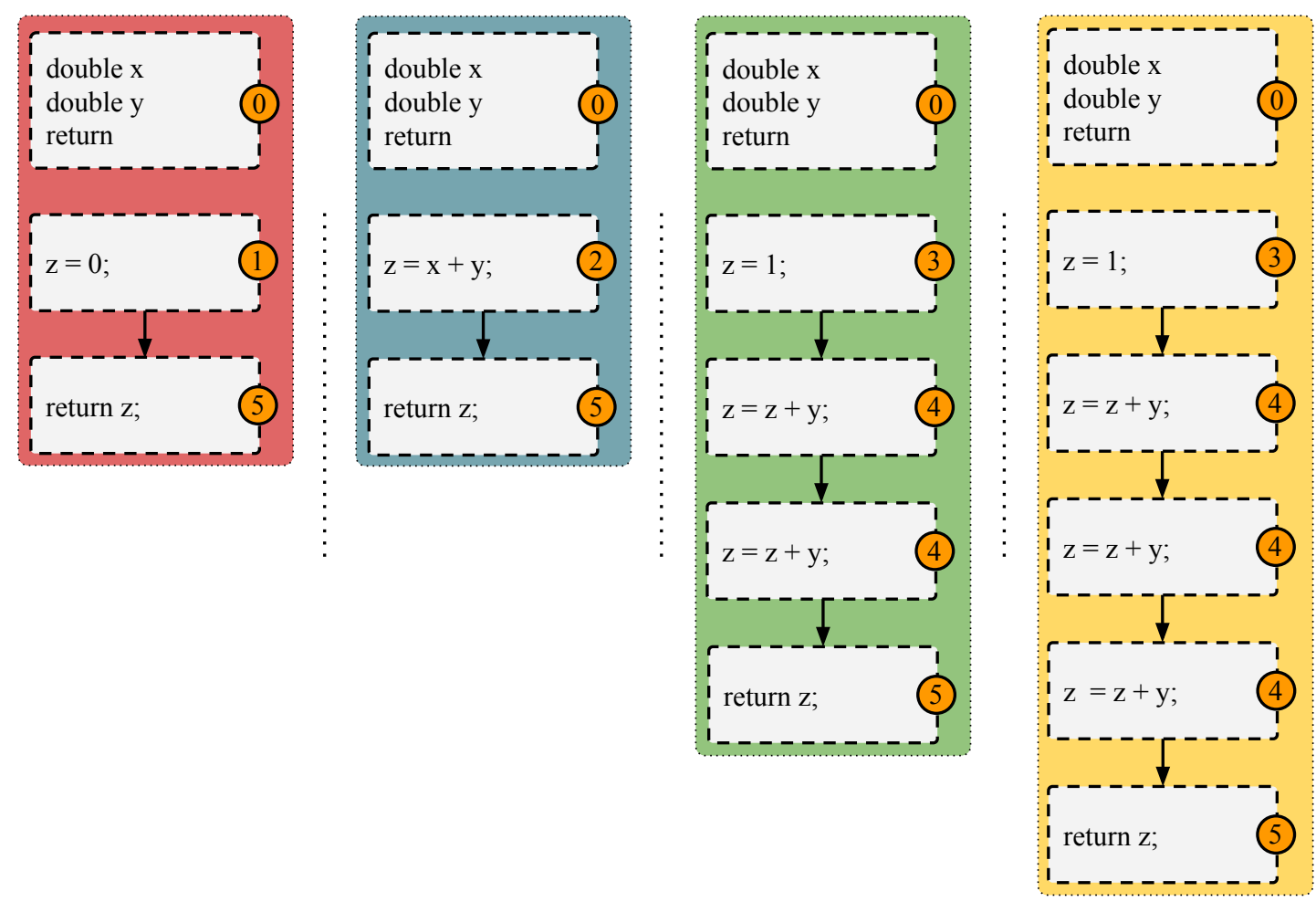

Fig. 4.9: Data Flow Graphs generated by the analysis of the nested conditionals case study.

Table 4.6: System statistics for different values of $J_{l i m}$ in the Selector case study.

\begin{tabular}{|c|c|c||c|c||c|c|}
\hline$J_{\text {lim }}$ & Partitions & Exec. Time & Mean & $\%$ error & Variance & $\%$ error \\
\hline \hline $10^{-2}$ & 103 & $4 \mathrm{~m} 2 \mathrm{~s}$ & 4.4120 & $0.18 \%$ & 4.9134 & $3.37 \%$ \\
\hline $10^{-3}$ & 226 & $7 \mathrm{~m} 4 \mathrm{~s}$ & 4.3983 & $0.12 \%$ & 5.0443 & $0.79 \%$ \\
\hline $10^{-4}$ & 964 & $43 \mathrm{~m} 32 \mathrm{~s}$ & 4.4064 & $0.05 \%$ & 5.0682 & $0.32 \%$ \\
\hline $10^{-5}$ & 3925 & $4 \mathrm{~h} 38 \mathrm{~m} 13 \mathrm{~s}$ & 4.4059 & $0.04 \%$ & 5.0802 & $0.08 \%$ \\
\hline
\end{tabular}

thus, deviates $0.05 \%$ and $0.32 \%$ from the expected values. The results for different values of $J_{l i m}$ are shown in Table 4.6 .

\subsubsection{Selector: RON modelling}

In this case study we require $7 \mathrm{RON}$ sources to model the quantization of the inputs, variables and output of the system. Following the naming convention from Section 4.3.2, we identify these noise sources as $\hat{e}_{x, 0}, \hat{e}_{y, 0}, \hat{e}_{z, 2}, \hat{e}_{z, 4.1}, \hat{e}_{z, 4.2}, \hat{e}_{z, 4.3}$ and $\hat{e}_{z, 5}$, and following the directives in Section 3.3.1 we assign 5 different $f_{w q}$ : 


$$
\begin{gathered}
\hat{e}_{x, 0}=\frac{2^{f_{x}}}{2} \epsilon_{1}, \\
\hat{e}_{z, 2}=\frac{2^{f_{z 2, i}}}{2} \epsilon_{3}, i=1,2,3, \\
\hat{e}_{z, 4 . i}=\frac{2^{f_{z 4}}}{2} \epsilon_{4}, \\
\hat{e}_{z, 5}=\frac{2^{f_{z 5}}}{2} \epsilon_{5} .
\end{gathered}
$$

We use a Monte-Carlo simulation ( $10^{7}$ samples) as our reference for a uniform quantization to 8 fractional bits, obtaining a variance $4.8460 \cdot 10^{-6}$ which, in turn, gives us a SQRN value $60.2086 \mathrm{~dB}$. Including the noise sources in each derivative subsystem and propagating the input coefficients for each domain partition generated by the methodology with $J_{l i m}=10^{-4}$ we obtain all the 964 partial results. We combine them following the equations in Sections 3.2.4 and 3.3.3 and we calculate a variance value $4.8478 \cdot 10^{-6}$ and a SQNR 60.1931dB.

\subsection{Clustered noise injection: L-Opchain}

Using FPGAs to accelerate scientific codes is a hot research topic $\left[\mathrm{BCL}^{+} 14\right]$ where fixed-point arithmetic plays a decisive role, given the need of implementing complex functionalities in hardware with strong area and speed constrains. These systems often have many inputs and large chains of operations that, when MAA/PCE is applied to estimate the RON, lead to scalability issues. In this case study we look at a system that, if approached directly, may pose a problem for the numerical engine trying to analyse it. Figure 4.10 shows the Data Flow Graph of the system, for which we consider all four inputs $a, b, c$ and $d$ to be $U[0,1]$. The PCE polynomials of the inputs, then, are:

$$
\hat{i}=0,5+0.5 \epsilon_{i}, \quad i=\{a, b, c, d\} .
$$

This case study show a particularly complex situation, as non-linear multiplications are costly operations which execution time degrades quickly as the size of the required base increases. The design has 4 inputs and 28 operations. Given the sequence of operations, in order to model the system accurately using MAA/PCE we need a base of order 4. This requires a base of 70 elements for the dynamic range estimation only. But were we to insert independent noise sources for all its inputs and operations at once, the number of sources would escalate up to 32 requiring a base of 58905 elements which, in turn, would generate a C matrix of roughly $2.04 \cdot 10^{14}$ elements. Propagating the PCE coefficients through a base of this size is a task highly demanding both in time and computational resources.

Instead, we follow the approach described in Section 3.4: to group the noises to inject in clusters. In this case study we set the maximum set size to 4 . In this way we 

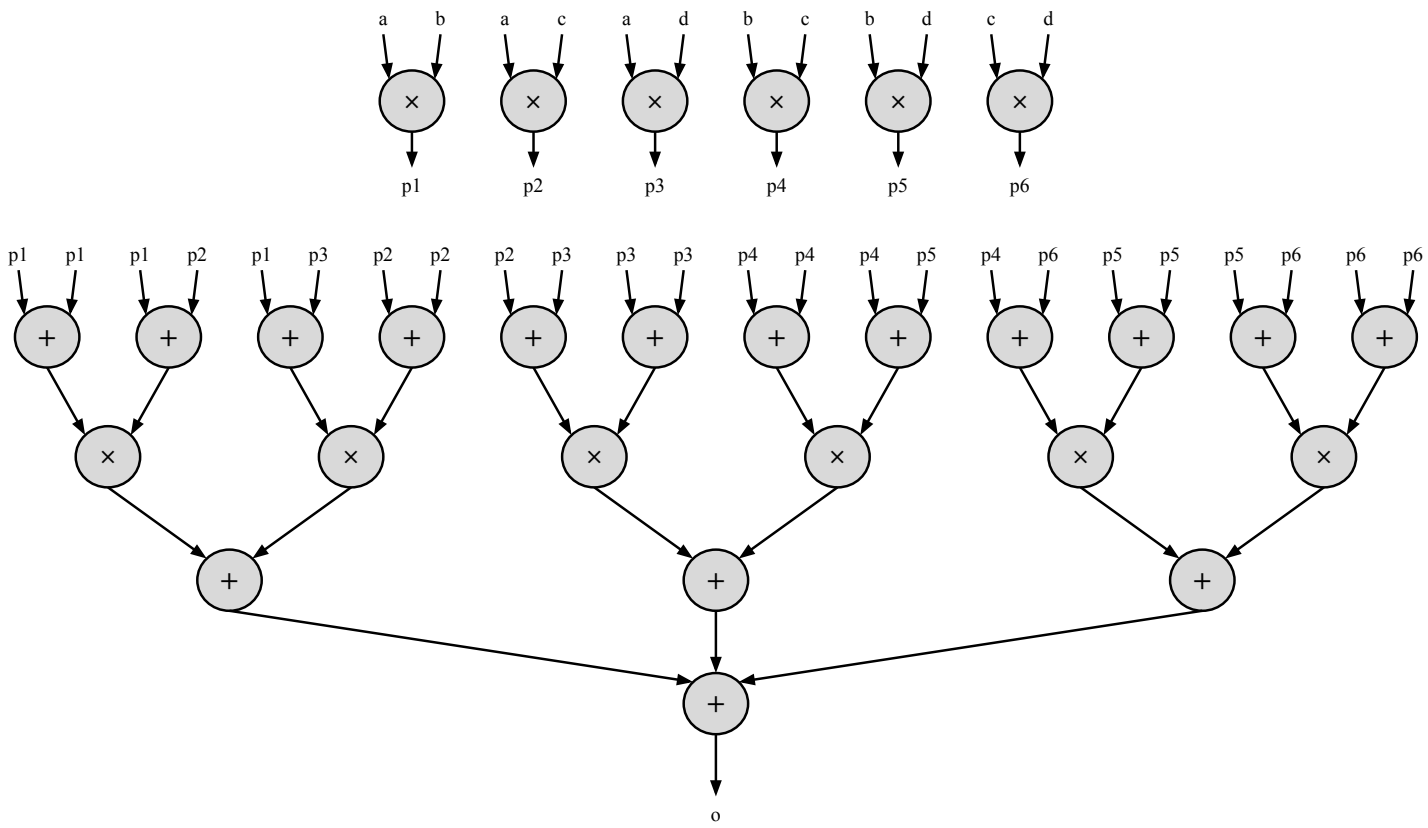

Fig. 4.10: L-Opchain case study DFG.

effectively limit the base size to 495 elements, merely $0.8 \%$ of the size required to include all noise terms at once. To carry out the partitioning we apply the hierarchical algorithm introduced in Section 3.4.3. This methodology tries to group the signals in balanced sets while minimizing the number of edges that cross between partitions. Following the steps described in Algorithm 2, our hierarchical partitioner generates 11 clusters in 5.21 seconds.

We propagate the affine input forms through the initial system to obtain an output PCE function of the signal inputs exclusively:

$$
\hat{o}_{s}=f(\hat{a}, \hat{b}, \hat{c}, \hat{d}) \text {. }
$$

Then, we generate the noise sources for every input and operation:

$$
\hat{n}_{i}=\frac{2^{f_{i}}}{2} \epsilon_{i}, i=[0-31]
$$

and we include the input and operation noise sources and study them independently in a per-cluster basis: For each cluster we take the initial system graph and we add the noise sources only to the operations in the cluster. Propagating the input PCE affine 
forms, we obtain a partial solution that contains signal and partial noise terms.

$$
\begin{gathered}
\hat{o}_{0}=f\left(\hat{a}, \hat{b}, \hat{c}, \hat{d}, \hat{n}_{0}, \hat{n}_{25}\right), \\
\hat{o}_{1}=f\left(\hat{a}, \hat{b}, \hat{c}, \hat{d}, \hat{n}_{3}\right), \\
\hat{o}_{2}=f\left(\hat{a}, \hat{b}, \hat{c}, \hat{d}, \hat{n}_{4}, \hat{n}_{7}\right), \\
\hat{o}_{3}=f\left(\hat{a}, \hat{b}, \hat{c}, \hat{d}, \hat{n}_{8}\right), \\
\hat{o}_{4}=f\left(\hat{a}, \hat{b}, \hat{c}, \hat{d}, \hat{n}_{10}, \hat{n}_{11}, \hat{n}_{12}, \hat{n}_{13}\right), \\
\hat{o}_{5}=f\left(\hat{a}, \hat{b}, \hat{c}, \hat{d}, \hat{n}_{15}, \hat{n}_{16}\right), \\
\hat{o}_{6}=f\left(\hat{a}, \hat{b}, \hat{c}, \hat{d}, \hat{n}_{17}, \hat{n}_{18}, \hat{n}_{19}, \hat{n}_{24}\right), \\
\hat{o}_{7}=f\left(\hat{a}, \hat{b}, \hat{c}, \hat{d}, \hat{n}_{14}\right), \\
\hat{o}_{8}=f\left(\hat{a}, \hat{b}, \hat{c}, \hat{d}, \hat{n} \hat{n}_{20}, \hat{n}_{21}, \hat{n}_{22}, \hat{n}_{23}\right), \\
\hat{o}_{9}=f\left(\hat{a}, \hat{b}, \hat{c}, \hat{d}, \hat{n}_{1}, \hat{n}_{5}\right), \\
\hat{o}_{10}=f\left(\hat{a}, \hat{b}, \hat{c}, \hat{d}, \hat{n}_{2}, \hat{n}_{6}, \hat{n}_{9}\right) .
\end{gathered}
$$

To retain the noise terms only, we subtract the PCE of the signal from each of the partial results:

$$
\hat{o}_{i, q}=\hat{o}_{i}-\hat{o}_{s}, i=[0-10],
$$

and we add all of them to the PCE of the signal to obtain the complete solution that includes all the signal and noise terms:

$$
\hat{o}_{q}=\hat{o}_{s}+\sum_{i=0}^{9} \hat{o}_{i, q}=\sum_{j=0}^{m-1}\left(\alpha_{s, j} \cdot \psi_{s, j}\right)+\sum_{i=0}^{9} \sum_{j=0}^{m-1}\left(\alpha_{i, j} \cdot \psi_{i, j}\right) .
$$

To obtain only the noise terms, we avoid adding the signal values:

$$
\hat{o}_{q}=\sum_{i=0}^{10} \hat{o}_{i, q}=\sum_{i=0}^{10} \sum_{j=0}^{m-1}\left(\alpha_{i, j} \cdot \psi_{i, j}\right) .
$$

Assuming a uniform quantization to 8 bits, we obtain our reference values through Monte-Carlo simulations ( $10^{7}$ samples): The simulation determines a noise variance of $1.7335 \cdot 10^{-4}$ and a SQNR of $45.2936 \mathrm{~dB}$. To extract the system statistics from Equation 4.16 we use the simplified form of the equations in Section 3.2.4 (i.e. no control-flow partitioning was required), which can also be found in works like $\left[\mathrm{ELS}^{+} 13\right]$ :

$$
\begin{gathered}
<\hat{o}_{q}>=\alpha_{0}, \\
\sigma_{\hat{o}_{q}}^{2}=\sum_{i=1}^{m-1}\left(\alpha_{i}^{2} \cdot<\psi_{i}^{2}>\right) .
\end{gathered}
$$

Applying these, we obtain a noise variance value $1.7275 \cdot 10^{-4}$ and a SQNR of 45.5352dB. The complete partitioning, propagation and computation of the noise statis- 
Table 4.7: Results for various cluster sizes.

\begin{tabular}{|c|c||c|c||c|}
\hline Size & Exec. time & Variance & $\Delta$ Variance & $\%$ error \\
\hline 1 & $743 \mathrm{~s}$ & $2.7805 \cdot 10^{-5}$ & - & $83.96 \%$ \\
\hline 2 & $530 \mathrm{~s}$ & $1.7021 \cdot 10^{-4}$ & $1.4241 \cdot 10^{-04}$ & $1.81 \%$ \\
\hline 3 & $489 \mathrm{~s}$ & $1.7275 \cdot 10^{-4}$ & $2.5495 \cdot 10^{-06}$ & $0.34 \%$ \\
\hline 4 & $515 \mathrm{~s}$ & $1.7275 \cdot 10^{-4}$ & $3.1475 \cdot 10^{-10}$ & $0.34 \%$ \\
\hline
\end{tabular}

tical moments take $87 \mathrm{~s}$ and it is fully automated. Table 4.7 shows experimental results for different maximum cluster sizes. There, the $\Delta$ Variance shows the difference respect to the previous variance value. It can be seen how using clusters of size one have a high impact on the output statistics and, given the large number of times the affine forms have to be propagated, a worse execution time. As the size increases, the deviation from the reference and the $\Delta$ are minimized.

\subsection{Putting it all together: Compounded}

In the previous case studies we have shown how to apply the control-flow PCE methodology to model the dynamic range and round-off noise of different systems (Sections 4.2 to 4.4). Then, in Section 4.5 we have applied the clustered noise injection methodology to approach the modelling of RON for a large system that presented scalability issues. In this case study we demonstrate how to combine both techniques.

Unifying the modelling of systems with control-flow structures and the clustered noise injection in a single flow allows us to maximize the range of applicability of the techniques we propose in this Ph.D. Thesis. The steps of the unified method are the following:

- Determine and generate the required derivative sub-systems (linear sequences of operations) from the initial system graph (which includes control-flow operations) and assign the corresponding domain partitions to each of them.

- Compute the dynamic range of the system using the equations in Section 3.2.4.

- Apply the clustered noise injection technique to each derivative sub-system independently. Calculate the output PCE for each sub-system following the steps in Section 3.4.1.

- Use the output results for each subsystem to estimate the RON in the complete system following the approach in Section 3.3.3.

In this section we follow the steps above to determine the dynamic range and the round-off noise of the system depicted in Figure 4.11. The system contains two controlflow structures and a total of 35 operations. Even though the derivative subsystems will not have all the operations on both branches of the choice operators at the same 


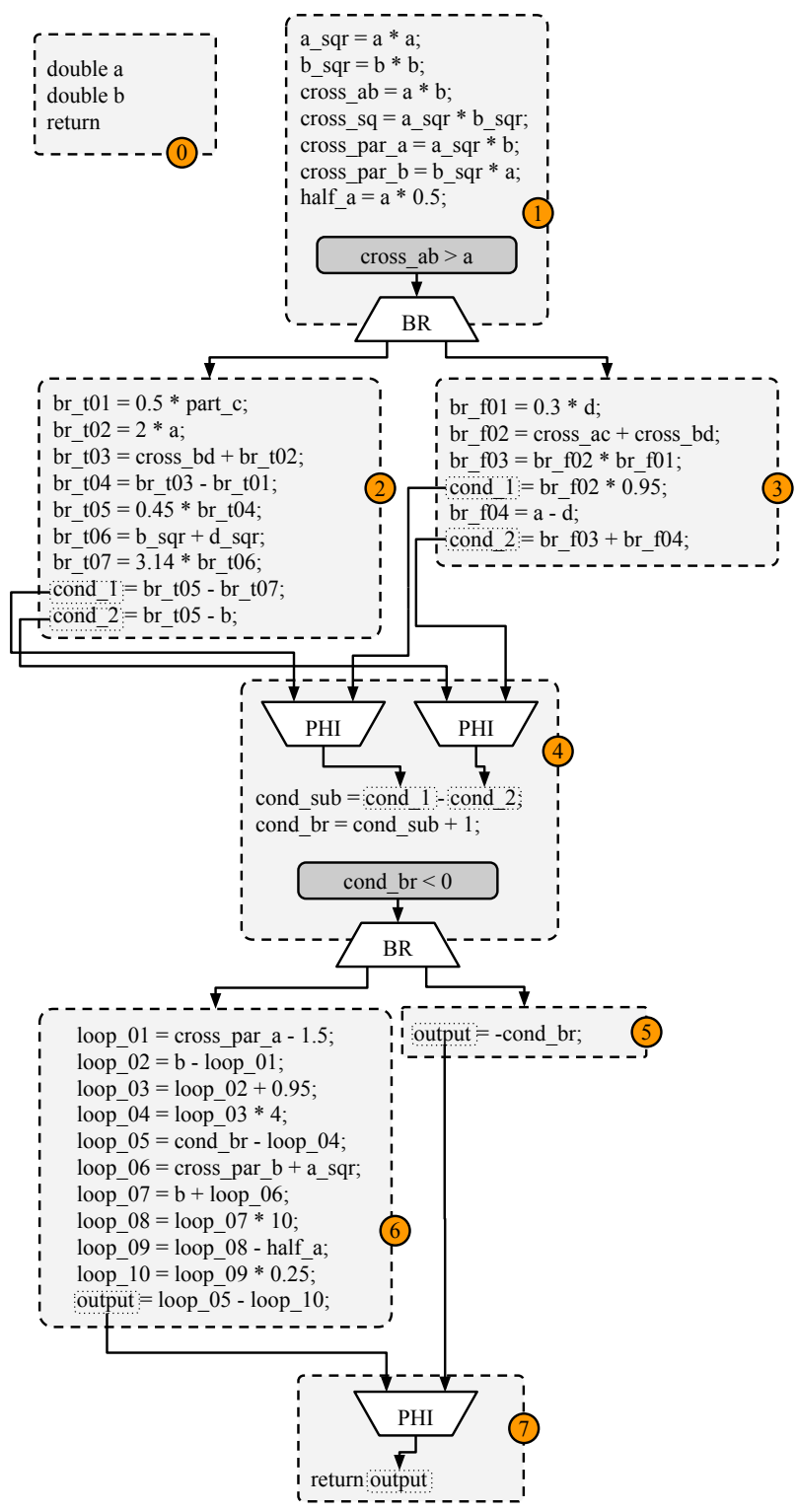

Fig. 4.11: Basic Blocks diagram for the compounded case study.

time, the size of the largest derivative subsystem is 29 operations. Given the sequence of operations, we use a fifth-order second-dimension Legendre PCE for this case study.

For the inputs we assume $a U[-2,1], b U[0,4]$. Also, we set the value of $J_{l i m}=10^{-4}$, we consider a maximum partition size of 4 for the clustered noise injection and we use a Monte-Carlo simulation with $10^{7}$ samples for reference.

\subsubsection{Compounded: Dynamic range estimation}

Given the distributions of the inputs, we determine their PCE polynomials:

$$
\begin{gathered}
\hat{a}=-0.5+1.5 \epsilon_{a}, \\
\hat{b}=2+2 \epsilon_{b} .
\end{gathered}
$$

As in the previous case studies, the first steps of the process are to determine the 
Table 4.8: Estimated system statistics for different values of $J_{l i m}$ in the Compounded case study.

\begin{tabular}{|c|c|c||c|c||c|c|}
\hline$J_{\text {lim }}$ & Partitions & Exec. Time & Mean & \% error & Variance & \% error \\
\hline \hline $10^{-2}$ & 112 & $9 \mathrm{~m} 38 \mathrm{~s}$ & 37.0007 & $2.08 \%$ & $1.1990 \cdot 10^{4}$ & $0.55 \%$ \\
\hline $10^{-3}$ & 265 & $22 \mathrm{~m} 15 \mathrm{~s}$ & 37.1937 & $2.61 \%$ & $1.1976 \cdot 10^{4}$ & $0.67 \%$ \\
\hline $10^{-4}$ & 1024 & $91 \mathrm{~m} 34 \mathrm{~s}$ & 36.5286 & $0.77 \%$ & $1.2035 \cdot 10^{4}$ & $0.18 \%$ \\
\hline $10^{-5}$ & 5026 & $418 \mathrm{~m} 5 \mathrm{~s}$ & 36.3460 & $0.27 \%$ & $1.2052 \cdot 10^{4}$ & $0.04 \%$ \\
\hline
\end{tabular}

partitions of the domain and to extract the derivative subsystems with the execution paths followed by values in those sub-domains. In this case study, after analysing all the control-flow structures, we obtain a total of 1024 partitions with their mappings distributed across the four derivative subsystems, as all combinations of decisions on the control-flow structures are exercised.

Once we have all the partitions mapped to their corresponding subsystems we apply the equations from Section 3.2.4 and we obtain a mean value of 36.5286 and a variance of $1.2035 \cdot 10^{4}$ which, compared to the corresponding reference values (obtained through Monte-Carlo simulations with $10^{7}$ samples that report a mean value of 36.2463 and a variance of $1.2057 \cdot 10^{4}$ ) present $0.77 \%$ and $0.18 \%$ deviation. Table 4.8 presents the results of the dynamic range computation for different values of $J_{k}$.

\subsubsection{Compounded: RON modelling}

To compute the RON we study each derivative subsystem independently and apply the equations in Sections 3.2.4 and 3.3.3 to obtain the statistical moments of the noise.

In this case study, the number of operations per subsystem ranges between 16 and 29 . We handle this number of noise sources applying the algorithm in Section 3.4.1 to each subsystem. Effectively, this means that every time we want to propagate the PCE coefficients of a sub-domain through its corresponding subsystem we will actually propagate them through several systems and them combine the results of each propagation into the actual partial result for that sub-domain. On average, the partitioner in Section 3.4.3 generates 8.5 partitions per subsystem, ranging from 6 partitions for the 16-operations subsystem to 11 partitions for the 29-operations one.

The Monte-Carlo simulation used as reference returns a noise variance of 0.1136 , which we use to obtain the SQNR value $50.2579 \mathrm{~dB}$. Once we propagate all the coefficients through their corresponding subsystems and we combine the clustered noises, we apply the relevant equations to obtain a variance of 0.1139 and, in turn, a SQNR of $50.2730 \mathrm{~dB}$.

\subsection{Conclusions}

In this chapter we have shown, through a selection of experiments, that the techniques introduced in Chapter 3 obtain accurate estimations for dynamic range and RON in non- 
linear systems that include control-flow operations, as well as in large systems where scalability issues can impose strict constraints regarding the number of independent inputs that can be included in the system at once.

The case studies in the chapter have been selected by their representation of typical scenarios that a developer will find every day when designing new systems. In the Teager + case study in Section 4.2 we tested our methodology against a non-linear system with a choice operator. The Accumulator in Section 4.3 introduces a loop structure, and the Selector in 4.4 combines both types of structures, nesting them. The L-Opchain case study in Section 4.5 does not include any control-flow operation, but instead shows the noise clustering process to achieve an accurate noise estimation in a system with high requirements in computational power and memory usage. Finally, in Section 4.6 we combine all the techniques to model both signal and RON in a large system with many control-flow structures. Across all the case studies, the methodologies we propose have obtained accurate estimations in manageable execution times, thus validating the methodologies we presented in Chapter 3 . 


\section{Chapter 5}

\section{Word-length optimization}

When you choose an action, you choose the consequences of that action. When you desired a consequence you had damned well better take the action that would create it.

Louis McMaster Bujold, Memory

With the increment in silicon area, as well in ASICS as in FPGAs, and the improvements in fabrication technology the main focus of WLO shifts towards finding good solutions quickly, even if they are not truly optimal. As such, greedy search algorithms are the best suited for this task, but as systems grow in complexity, the solution space that WLO methods have to explore expands exponentially and classical methods fail to deliver results fast enough. This is specifically true when considering simulation-based approaches. While analytical approaches (like the ones presented in the previous chapter) spend most of the computation time in the modelling phase in order to allow fast word-length optimization, simulation-based approaches skip the modelling phase at the cost of very long computation times during optimization.

In this chapter we explore different solutions to speed-up the execution time of WLO searches when simulation-based methods are used to characterize the RON. The two contributions presented here are greedy techniques that aim at reducing the execution time required to find suitable solutions when Monte-Carlo simulations are used to evaluate the impact of the round-off noise. This allows applying the proposed approaches to every type of system.

The first contribution, presented in Section 5.2, introduces an interpolative method that saves intermediate sensitivity values in early stages of the optimization process and uses them to guide the subsequent search. After presenting the methodology, we discuss how the number of simulations can be minimized without serious impact on the quality of the obtained solutions and then we detail its application to a case-study. 
Section 5.3 describes an incremental method where speedup is achieved by means of initializing the search with a relaxed confidence interval and iteratively refining the solutions with tighter intervals. In this section, we explain confidence intervals and relate them with the number of required Monte-Carlo simulations. Then we describe the incremental method in detail and we apply it to a case-study. A complete set of experimental results is presented in Section 5.5.

\subsection{Definitions}

In this chapter we will refer to some classical approaches to WLO and we will use some terms commonly used across the WLO literature. Even though we have described some of them briefly in Chapter 2, in this section we define these terms in detail before describing the new WLO methodologies we propose.

- mUWL: The first step in several optimization algorithms is to do a coarse-grain optimization to rule out all those word-length configurations that are well far from the optimal values. This is often done by looking for the smallest uniform wordlength vector (i.e. all variables have the same size allocated) that complies with the imposed constraint. We call this word-length the Minimal Uniform Word-Length.

- mWS: In order to limit the solution space to explore, we want to set bounds for it. And while the mUWL acts as an upper bound, we also need to set lower bounds. To do that, we search the minimum word-length for each variable that satisfies the constraints while the rest of the variables either have an arbitrarily long word-length (floating-point) or, more commonly, are set to the mUWL. In order to speed-up this search process, we often reduce $b>1$ bits in each step. The word-length vector resulting from combining all those individual minima is what we call the Minimal Word-length Solution which, in most cases, does not comply with the noise constraints.

- $\min +1$ search: It is a classical greedy procedure consisting of three phases. First, we perform a uniform search to obtain a mUWL. Then, starting from that result, we search the mWS, thus enclosing the solution space between the two boundaries. The third and final step is an iterative competition among all the variables, starting from the mWS, to increase their size, one bit at a time. In each iteration of the competition, the bit that causes a greater reduction in the error is retained. A known issue with the $\min +1$ search is that it may get trapped in local minima.

- Preplanned search: It is another greedy procedure, this one proceeding in four execution phases. First, we perform a uniform search to obtain a mUWL. Then, we compute the sensitivity of each variable while we obtain the mWS. To do this, we search the minimum word-length of each variable with the mUWL assigned to the rest of them, using decrements of 1 bit each time, computing the sensitivity as 
$s_{i}=f\left(W L_{i}+1\right)-f\left(W L_{i}\right)$, being $f(x)$ an objective function and $W L_{i}$ the mWS for the variable, , and saving it for later use. Once that is finished, we construct a global priority list in decreasing order of sensitivities. Finally, guided by this list, we carry out an iterative competition where, in each step, the variable with the largest sensitivity is incremented by one bit. This competition continues until we find a word-length configuration that complies with the noise constraints.

\subsection{Interpolative method}

The preplanned search [HEKC01] is one of the fastest strategies for WLO. Nevertheless, a large amount of simulations is still required to obtain the sensitivity information used to perform the preplanning and subsequent search. Our method is based on the preplanned search and applies a simple but precise interpolator to reduce the number of simulations required to extract the sensitivity values that are used to guide the search $\left[\mathrm{SLC}^{+} 12 \mathrm{~b}\right]$. We also consider the limit case where the number of simulations during the sensitivity values gathering stage is reduced down to two evaluations per variable. The experimental results for this method show speedup values between 1.59 and 11.86 for small and medium-sized problems.

\subsubsection{Methodology}

The interpolative method is decomposed in three steps:

1. Compute a suitable mWS for the system considering $b$ bits reductions. During this process, noise values obtained for each individual variable when the rest of them have an arbitrarily high precision (i.e. use a floating-point representation) are saved for later use.

2. Interpolate the remaining noise values from those computed in the previous step and obtain the differences between values.

3. Explore the solution space through bit-increments of the variables with the steepest noise reductions until the system noise constraints are met.

Figure 5.1 presents a block diagram of the complete process. The remaining parts of this section detail the steps involved the interpolative method, the problem of the optimistic estimation, and its solution.

\section{Search of the minimum word-length solution}

First, we search for a suitable mWS following the method proposed in [SK95] applying $b$ bits reductions. The noise values for individual variables that we obtain during this search are stored in a $v \times w$ matrix $\left(M_{e}\right)$. Here, $v$ is the number of variables to be quantized and $w$ is the maximum word-length allowed. Thus, position $M_{e}(i, j)$ stores the output 


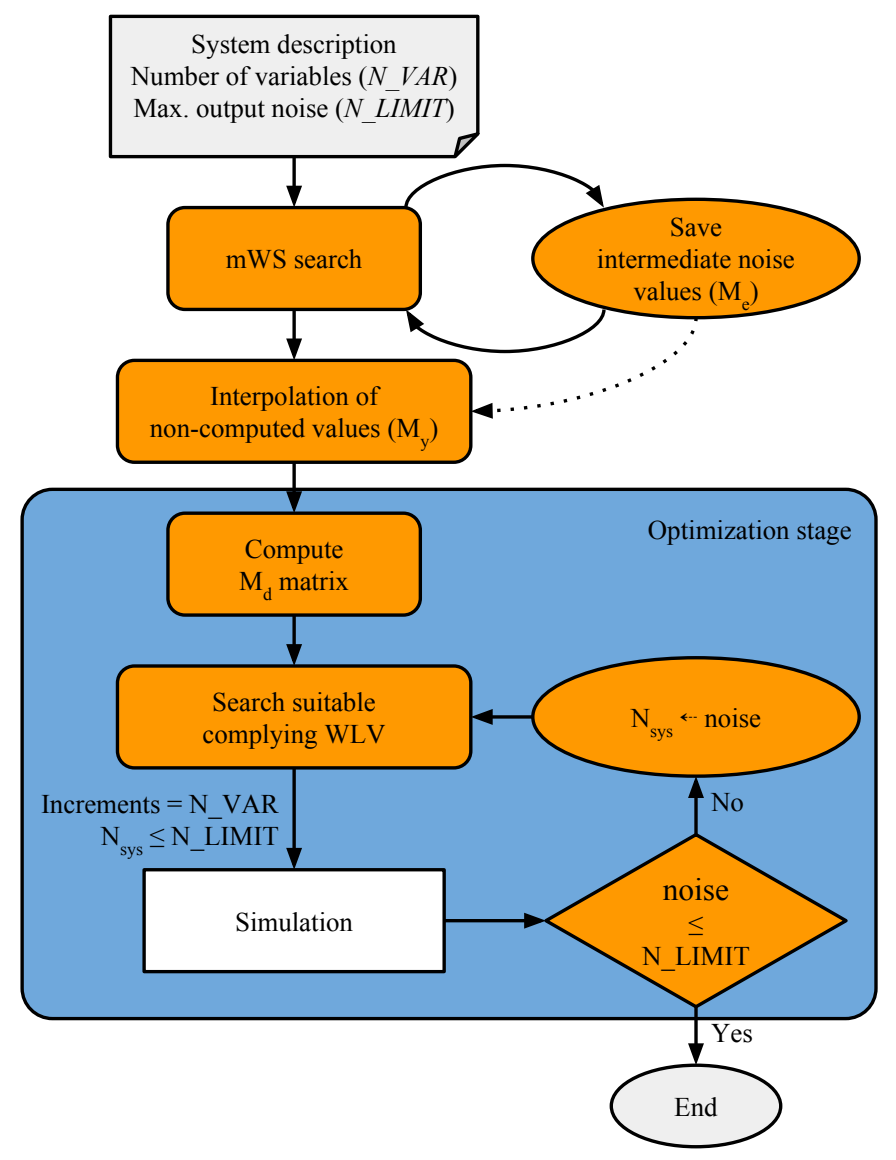

Fig. 5.1: Interpolative method algorithm flow.

noise power generated by the $i$-th variable when its fixed-point representation uses $j$ bits and the rest of the system uses arbitrarily long floating-point arithmetic.

\section{Interpolation of the non-computed noise values}

Since the search for the minimum word-lengths of each variable is done by reducing its width $b$ bits each time, $M_{e}$ matrix has undetermined positions that must be filled before further computations can be carried out. For this purpose, we perform an interpolation based on the properties of linear and smooth non-linear systems, in which the variance of the round-off noise of a variable is proportional to $2^{-w}$ being $w$ the allocated number of bits for the fractional part of the fixed-point variable [Pah99]. This implies that, by increasing the fractional word-length in one bit we decrease the round-off error by a factor of 4 . The results of this interpolation are used to fill a new matrix $M_{y}$ of the same size of $M_{e}$. The interpolation is based on the following criteria:

$$
M_{y}(i, j)= \begin{cases}M_{e}(i, j), & \text { if }(i, j) \in S_{c} \\ \frac{M_{e}(i, j-p) * 4^{p}+\frac{M_{e}(i, j+q)}{4^{q}},}{2}, & \text { otherwise. }\end{cases}
$$




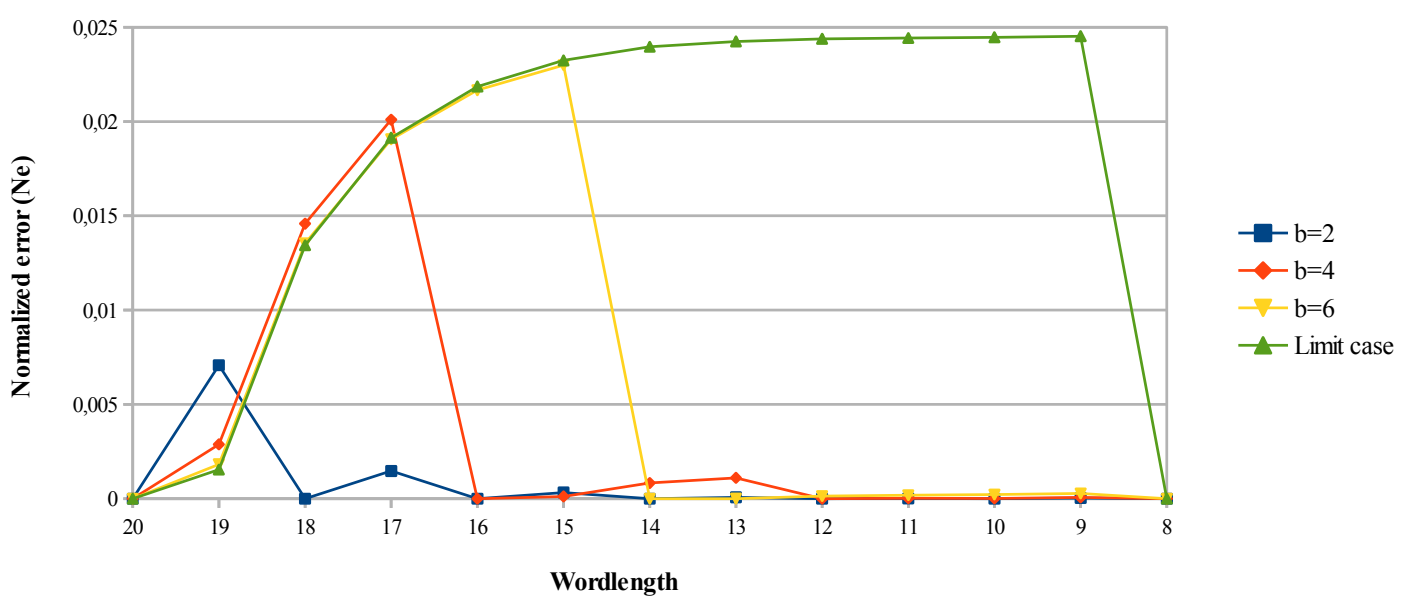

Fig. 5.2: Evolution of normalized error for different values of $b$.

where $S_{c}$ is the set of all the pairs $(i, j)$ for which $M_{e}(i, j)$ was computed in the previous step. $(i, j-p)$ and $(i, j+q)$ are the nearest lower and upper elements to $(i, j)$ that belong to $S_{c}$, respectively. Consequently, $p$ and $q$ are the distances to the closest elements in the same $M_{e}$ column that have already been obtained through simulation.

Figure 5.2 shows how the estimation of the output noise deviates from the real noise values using different values of $b$ when we apply Equation (5.1). In all cases the noise values for word-lengths 20 and 8 were obtained through MC.

Being $x^{*}(k)$ the actual noise value obtained through simulation for word-length $k$ and $x_{b}(k)$ the value obtained through the interpolative method for the given value of $b$, each value in the curves of Figure 5.2 is obtained as

$$
N_{e}(k)=\frac{\left|x^{*}(k)-x_{b}(k)\right|}{x_{b}(k)} .
$$

In the experiments carried out with $b=2$ the differences between the simulated and the interpolated values were always at least two orders of magnitude smaller than the values themselves. Thus, we can consider that the effect of these errors in the process is negligible.

\section{Search of a complying solution}

This part of the algorithm is divided in several smaller tasks that are detailed as follows:

- Computation of the $M_{d}$ matrix

Matrix $M_{y}$ contains the individual noise contributions of each variable to the output noise. We can use this information to select the word-lengths that must be increased in order to comply with the noise requirements. To do so, we define 
matrix $M_{d}$ of size $v \times w$. It represents the estimated noise variations when each signal word-length is modified. Specifically, position $M_{d}(i, j)$ indicates the noise decrement when incrementing the word-length of variable $i$ from $j$ to $j+1$ bits. Being $w_{\min }(k)$ the minimum word-length for the $k$-th variable as computed in the first step of the algorithm, the values of $M_{d}$ are:

$$
M_{d}(i, j)= \begin{cases}0, & \text { if } j=w \\ M_{e}(i, j)-M_{e}(i, j+1), & \text { if } w_{\text {min }}(i) \leq j<w .\end{cases}
$$

In essence, matrix $M_{d}$ provides a quantitative description of the sensitivities of the variables to the variation of their word-lengths.

- Search and noise estimation

Next, we perform a simulation with all the variables adjusted to their $w_{\min }$ value to obtain the actual noise level of the system, $N_{\text {sys }}$. Those word-lengths with the highest value in matrix $M_{d}$ are iteratively incremented and the noise level updated by subtracting the noise difference value from $N_{\text {sys }}$ until the computed noise level complies with the requirements.

- Overall estimation adjustment

While the proposed method reduces the number of simulations to perform, its estimations tend to be optimistic. The noise reduction produced by the increment of a given word-length is often lower than the corresponding $M_{d}$ value. Thus the estimated error complies the specification while the real quantized system does not. To overcome this issue, we perform a simulation every $n$ bit increments in order to obtain the actual output noise and adjust the $N_{\text {sys }}$ value accordingly. We have empirically observed that selecting a value of $n$ equal to the value of the number of variables to be quantized offers a good trade-off between the number of additional simulations and the deviation of the estimation from the actual noise values. Also, once the estimation has determined that the WLV complies with the noise margins, it is necessary to simulate the system to determine if further processing is necessary.

\subsubsection{Minimization of the number of simulations}

As we indicated previously, the mWS is searched by reducing the word-length of each variable by $b$ bits in each step. Equation (5.1) interpolates the missing values of $M_{e}$ using the results of the simulations performed until the mWS values are obtained. In order to minimize the number of performed simulations, it is possible to modify the first step of the algorithm. We need fewer simulations as we increase the value of $b$. In the limit case, we would only require two simulations for each variable: one for the default word-length and another for the mWS value. 


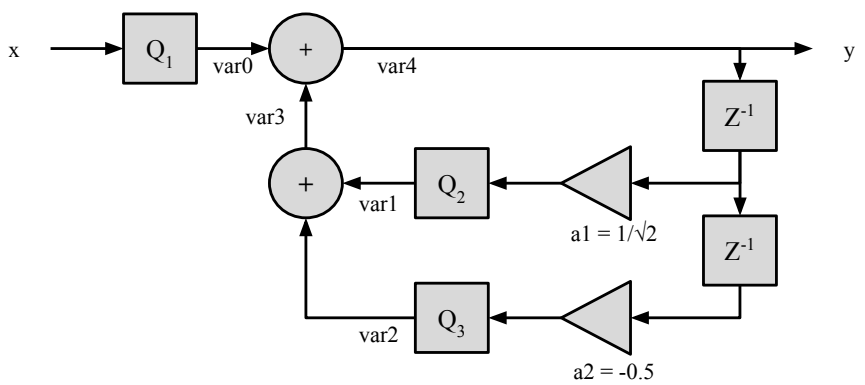

Fig. 5.3: Block diagram of a second order IIR filter.

Considering this limit case, an issue arises: we do not know the mWS value of each variable in advance, and we only discover it when we simulate and get the actual wordlength. To overcome this, it is possible to use an approximation instead of the mWS: the noise level of each variable is estimated by $N_{w l}(i, j)=N_{w l}(i, j+1) * 4$, being $N_{w l}(i, j)$ the noise level for the variable $i$ with word-length $j$. This can be used to study the evolution of the noise level of each variable until a given $N_{w l}(i, k)$ that goes over the system noise limit is found, being $N_{w l}(i, k+1)$ a compliant value. Then, another simulation is performed with word-length $k$. In this way, we obtain maximum and minimum noise values. From these, we can apply equation (5.1) to supply data for $M_{d}$. Once the matrix is filled, we can continue with the process as described in Section 5.2.1, step 2.

\subsubsection{Interpolative: Case study}

The second-order IIR filter shown in Figure 5.3 is extracted from [LCCNT08] and used as case study. For this example, we consider an upper limit for the output noise $\sigma^{2}=3.3 \cdot 10^{-11}$. After the uniform quantization, we perform the search for the mWS. In this search the individual noise contributions of each variable are annotated in the $M_{e}$ matrix as explained in section 5.2.1.

Once the process finishes, $M_{y}$ is computed according to equation (5.1). Afterwards, we obtain $M_{d}$ applying equation (5.3). We show its content in Table 5.1. Word-lengths that have not been analysed (since the noise variance was above the system limit) are indicated with the "-" mark. Underlined values indicate the word-lengths of the mWS. Also, since variables with word-lengths lower than 15 do not comply with the system noise constraint, no information about them is presented.

As we indicated previously, a position like $M_{d}(\operatorname{var} 2,17)$ means that if the word-length of var 2 is increased from 17 to 18 bits, the output noise variance will decrease by 2.264 . $10^{-11}$. The information in $M_{d}$ is used to iteratively select the variable with the maximum noise reduction and increase its word-length. As said before, every $n$ increments a new simulation is performed to adjust the noise power value to its real value. In this case a value $n=5$ is considered. The evolution of the interpolative method is shown in Table 5.2. The Action column indicates the operation performed in each iteration of the algorithm. A simulation is performed (Evaluate result) when the estimation of the noise 
Table 5.1: $M_{d}$ matrix for a second-order IIR filter.

\begin{tabular}{|c|c|c|c|c|c|}
\hline Width & var0 & var1 & var2 & var3 & var4 \\
\hline \hline 19 & 0 & 0 & 0 & 0 & 0 \\
\hline 18 & $1.393 \cdot 10^{-12}$ & $5.647 \cdot 10^{-12}$ & $5.660 \cdot 10^{-12}$ & $5.647 \cdot 10^{-12}$ & $\underline{2.254 \cdot 10^{-11}}$ \\
\hline 17 & $5.622 \cdot 10^{-12}$ & $\underline{2.259 \cdot 10^{-11}}$ & $\underline{2.264 \cdot 10^{-11}}$ & $\underline{2.259 \cdot 10^{-11}}$ & $9.017 \cdot 10^{-11}$ \\
\hline 16 & $\underline{2.250 \cdot 10^{-11}}$ & $9.036 \cdot 10^{-11}$ & $9.056 \cdot 10^{-11}$ & $9.037 \cdot 10^{-11}$ & - \\
\hline 15 & $9.008 \cdot 10^{-11}$ & $3.615 \cdot 10^{-10}$ & $3.622 \cdot 10^{-10}$ & $3.614 \cdot 10^{-10}$ & - \\
\hline
\end{tabular}

Table 5.2: Evolution of the interpolative method for a second-order IIR filter.

\begin{tabular}{|c|c|c|c|c|}
\hline Iter. & Action & WLV & Noise $\sigma^{2}$ & Result \\
\hline \hline 0 & Initialization & {$[16,17,17,17,18]$} & $9.0612 \cdot 10^{-11}(\mathrm{~s})$ & Fail \\
\hline 1 & Increment var2 & {$[16,17,18,17,18]$} & $6.7971 \cdot 10^{-11}(\mathrm{e})$ & Fail \\
\hline 2 & Increment var1 & {$[16,18,18,17,18]$} & $4.5377 \cdot 10^{-11}(\mathrm{e})$ & Fail \\
\hline 3 & Increment var3 & {$[16,18,18,18,18]$} & $2.2786 \cdot 10^{-11}(\mathrm{e})$ & Pass \\
\hline 4 & Evaluate result & {$[16,18,18,18,18]$} & $6.5501 \cdot 10^{-11}(\mathrm{~s})$ & Fail \\
\hline 5 & Increment var4 & {$[16,18,18,18,19]$} & $4.2958 \cdot 10^{-11}(\mathrm{e})$ & Fail \\
\hline 6 & Increment var0 & {$[17,18,18,18,19]$} & $2.0454 \cdot 10^{-11}(\mathrm{e})$ & Pass \\
\hline 7 & Adjust estimation & {$[17,18,18,18,19]$} & $2.2647 \cdot 10^{-11}(\mathrm{~s})$ & Pass \\
\hline
\end{tabular}

variance goes below the system constraint in order to determine if the result is valid or not. After $n$ bit increments a simulation is performed (Adjust estimation) with MC. In the Noise $\sigma^{2}$ column, two letters can be observed besides the variance value, indicating whether they have been obtained through (s)imulation or (e)stimation. The search will not stop until the variance obtained through simulation is below the noise limit.

The simulation time required to quantize this 5 -variable problem following the steps presented in Section 5.2.1 was 66 minutes, while the preplanned search took 105 minutes to reach a solution and the min +1 scheme needed 160 minutes. If the first step of the interpolative algorithm is modified to work as explained in Section 5.2.2, the overall computation time descends to 48 minutes. Therefore, we achieve speedups of x1.59 and x2.18 with respect to the preplanned strategy are achieved. Additionally, no degradation is observed in the obtained WLV.

\subsection{Incremental method}

When searching an adequate solution for the WLO problem, evaluating every possible WLV configuration involves a high number of simulations to ensure that the noise error 
obtained is within a reasonable confidence interval. While it is strictly necessary to guarantee that the solution given by the algorithm is bound to a confidence interval small enough, this constraint can be relaxed during the first steps of the search [SLC $\left.{ }^{+} 12 \mathrm{a}\right]$. By means of this, a feasible complying WLV is approximated with a reduced number of simulations. Afterwards, by narrowing the confidence interval, we refine the solution until we comply with the confidence interval.

\subsubsection{Confidence intervals and number of simulations}

Before detailing the functioning of the second proposal, it is necessary to give a brief explanation on the rationale behind it. As shown in [ZB97] and [Jer84], the number of simulations needed to guarantee that a solution is correct is intimately related to the resolution of the noise constraint in the system. Being $\alpha$ the allowed error given as a fraction of unity, we can define an envelope around the mean of the signal for which the probability of the simulated values to be inside the said envelope is $1-\alpha$. This is called the confidence interval of the simulation, and is defined as $I=\left(y_{-}, y_{+}\right)$, where

$$
y_{ \pm}=\hat{p}\left\{1+\frac{d_{\alpha}^{2}}{2 N \hat{p}}\left[1 \pm \sqrt{\frac{4 N \hat{p}}{d_{\alpha}^{2}}+1}\right]\right\}
$$

being $\hat{p}$ the desired precision of the simulation, $N$ the number of performed simulations, and $d_{\alpha}$ a value chosen so that

$$
\int_{-d_{\alpha}}^{d_{\alpha}} \frac{1}{\sqrt{2 \pi}} e^{-\frac{t^{2}}{2}} d t=1-\alpha
$$

The interested reader can find justification and further details on the confidence interval in [Jer84]. As stated there, a confidence interval $I_{r e f}=(0.5 \hat{p}, 2 \hat{p})$ is usually considered precise enough. In the characterization of a given system, the number of simulations needed to bound the error to $I_{\text {ref }}$ must be at least $10 / \Theta(\hat{p})$. This means that when the noise constraint is of order $10^{-k}$, we must perform at least $N=10^{k+1}$ simulations to consider the result as valid.

It must be noted that the precision of the estimation depends exclusively on the number of points used for such estimation, and not on the value to be estimated. However, if the value to estimate is very small it is important to that the confidence of the estimation is at least of the same order as the value to estimate. The aforementioned references refer directly to Bit Error Rate (BER). This means that, by having that number of points as samples, the estimations of the mean and variance are within the computed bounds with a certain probability.

By reducing the number of simulations, the confidence interval of the estimation widens. In this sense, if $N=10^{k}$, the interval opens up to $(0.1 \hat{p}, 8 \hat{p})$. When only $N=10^{\frac{k}{2}}$ simulations are performed, the confidence interval extends to $\left(1.5 \cdot 10^{-\left(\frac{k}{2}+1\right)} \hat{p}, 5 \cdot 10^{\frac{k}{2}} \hat{p}\right)$. A value as imprecise as this should not be used to take definitive decisions about word- 
length selection. Nevertheless, we can use it to obtain preliminary results that can be later verified or modified applying smaller confidence intervals.

\subsubsection{Methodology}

Once we have introduced the relationship between the number of simulations and the confidence in the solutions, we can explain the algorithm. The incremental method has three main steps:

1. Search for a suitable mUWL and a mWS with a relaxed confidence interval.

2. Starting from a relaxed confidence interval, iteratively carry out greedy searches to find the best solution and tighten the confidence interval until the search is done with $I_{r e f}$.

3. Check the obtained results with the reference confidence interval and, if possible, refine the solution further.

We present the complete process in Figure 5.4. In the figure, the boxes marked as "Greedy search" involve also deciding the type of search that will be carried out. This is explained in the following paragraphs, where we detail each of the steps of the incremental process. From now onwards, we will assume $10 / \Theta(\hat{p})=10^{k_{r e f}}$.

\section{Search for suitable mUWL and mWS}

As in many classical algorithms like min +1 [CSL02], the first step of this procedure is to determine a suitable initial set of word-lengths for the later search. To speed up this initial search, first we will search the mUWL that makes the output noise comply with the design constraints, and from that solution we obtain the mWS.

But the mWS seldom shares a result with the final solution, and in most cases all the variables will see a change in the number of bits they need to allocate from that initial WLV. Thus, having a very precise mWS is not critical for the process. And even so, an important share of time is devoted to this task. In the incremental method, instead, this search is carried out with a relaxed confidence interval. These searches will be done using only $N=10^{k_{1}}$ simulations to characterize each WLV, being $k_{1}=\left\lfloor\frac{3 \times k_{r e f}}{4}\right\rfloor$. Doing this, the execution time of these initial stages is noticeably decremented with negligible, if any, impact on the rest of the process.

\section{Incremental interval tightening and search refinement}

The main idea behind the incremental method is that we can infer the main direction of the WLO through rough estimations, discarding a fair number of WLVs with minimal simulation effort. We can then refine the search progressively, performing a larger number of simulations in much reduced solution spaces, until we obtain a suitable solution verified with the required level of detail (i.e. a confidence interval small enough). 


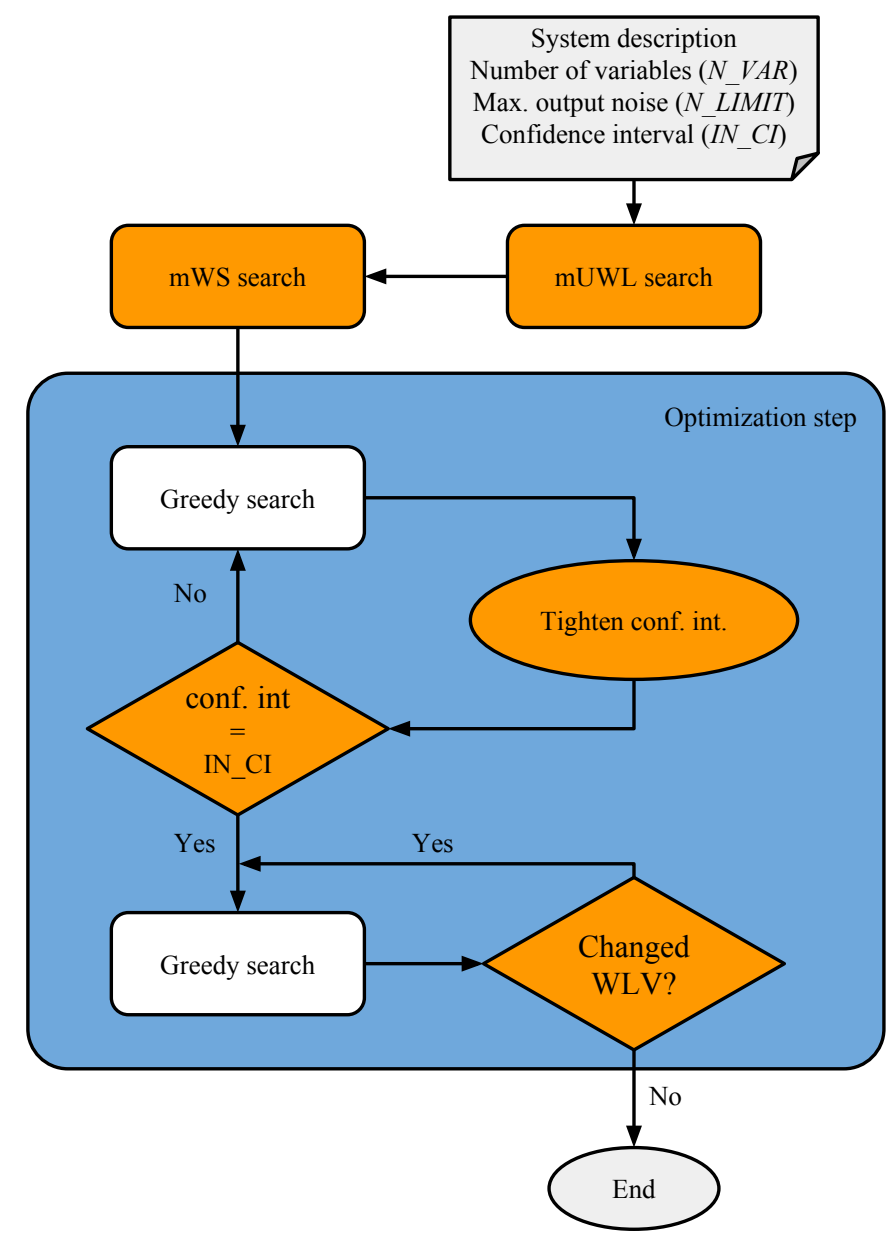

Fig. 5.4: Incremental method algorithm flow.

To do this, we will first consider a relaxed confidence interval and test each WLV using only $N=10^{\left\lfloor\frac{k_{r e f}}{2}\right\rfloor}$ random input values. Starting from the mWS obtained in the previous step, all variables will iteratively compete for increasing one bit their wordlength in a greedy $\min +1$ search. Once that search is complete, we tighten the considered confidence interval and evaluate the obtained solution.

If the confidence interval is not yet the reference one, we will do a new greedy search starting from the solution in the previous iteration. In case the first evaluation in the new confidence interval reported a solution compliant with the noise budget, we will proceed with a search where variables will compete to reduce their length in a max-1 style. In case the evaluation indicated a non-compliant solution, the competition will be of a $\min +1$ nature. Each time the interval is tightened, we will set the number of simulations to $N=10^{k_{2}}$, being $k_{2}=\left\lfloor\frac{k_{\text {prev }}+k_{\text {ref }}}{2}\right\rfloor+1$ where $k_{\text {prev }}$ is the order of the number of simulations performed in the previous iteration. We will repeat the process until the compliance of the output noise is verified within the confidence margin $I_{r e f}$ (i.e. with $N=10 / \Theta(\hat{p})$ simulations performed).

This scheme adapts efficiently to the required order of $\hat{p}$. If the required precision is 
small, the successive values of $N$ will be close to each other and the search will be guided efficiently, since the number of simulations needed to evaluate each configuration will not be prohibitively high. But if the required precision is large, configurations with much wider confidence intervals will be considered in the beginning, reducing the required number of solutions to test in the last steps of the search, thus reducing the number of required simulations and the overall execution time.

\section{Search within reference confidence interval}

Once we reach the reference confidence interval we can still find optimization opportunities. Thus, we will continue refining the solution using $10 / \Theta(\hat{p})$ simulations to evaluate each WLV. The direction of the first greedy search in the reference confidence interval is decided by the result of the last evaluation in the previous step: If the WLV complies with the error bounds, we will carry out a $\max -1$ search. Otherwise, the search will be a min +1 type.

Afterwards, we will alternate the two types of greedy searches similarly to the hybrid procedure [CSL02] until the selected WLV is no longer changed. This way we can take advantage of any possible optimization opportunity, even though empirical results show that the solution usually converges in one or two iterations of the loop.

\subsubsection{Incremental: Case study}

We will use the same second-order IIR filter as in Section 5.2.3, which is shown in Figure 5.3. For this five-variable case study we set the noise upper limit to $\sigma^{2}=$ $3.3 \cdot 10^{-5}$. Accordingly, to obtain results within a sufficient confidence interval we set $k_{r e f}=6$. Following the steps detailed in Section 5.3.2, we first search a mUWL. In this step, the quantization noise of each WLV is evaluated using $10^{4}$ simulations, which gives the confidence margin $\left(3 \cdot 10^{-7}, 2 \cdot 10^{-4}\right)$ for the results. We find the mUWL after seven iterations and then we start the evaluation of the mWS with the same number of simulations per evaluation.

Once we find the mWS with that confidence margin, we set the variables to compete to increase their word-length iteratively. Since it is the beginning of the fine-grain optimization, we set the number of simulations per evaluation to $10^{3}$ which, as indicated in Section 5.3.1, gives solutions within a confidence interval $\left(3.8 \cdot 10^{-8}, 2.6 \cdot 10^{-3}\right)$. After five bit increments the simulation determines that the WLV complies with the noise constraint.

Following the algorithm and given $k_{\text {prev }}=10^{3}$ and $k_{r e f}=10^{6}$, we increase the number of simulations to $10^{5}$. The output noise for this WLV is computed and found to meet the noise constraint, so a competition among the variables to decrement one bit is carried out. In this case, the evaluations determine that if any word-length is reduced the system will no longer comply with the constraint and the competition stops. We increase the number of simulations to $10^{6}$, thus tightening the confidence interval to the 
Table 5.3: Distribution of the execution times among the optimization stages.

\begin{tabular}{|c|c|c|}
\hline Stage & min+1 & Incremental \\
\hline \hline mUWL & $24^{\prime} 8^{\prime \prime}\left(N=10^{6}\right)$ & $10^{\prime \prime}\left(N=10^{4}\right)$ \\
\hline mWS & $37^{\prime} 12^{\prime \prime}\left(N=10^{6}\right)$ & $18^{\prime \prime}\left(N=10^{4}\right)$ \\
\hline & & $4^{\prime \prime}\left(N=10^{3}\right)$ \\
Competition & $99^{\prime} 3^{\prime \prime}\left(N=10^{6}\right)$ & $4^{\prime} 34^{\prime \prime}\left(N=10^{5}\right)$ \\
& & $19^{\prime} 38^{\prime \prime}\left(N=10^{6}\right)$ \\
\hline Total & $160^{\prime} 23^{\prime \prime}$ & $24^{\prime} 40^{\prime \prime}$ \\
\hline
\end{tabular}

reference $(0.5 \hat{p}, 2 \hat{p})$.

We evaluate the solution obtained in the previous step with this number of simulations and we verify that the obtained output noise complies with the upper noise limit of the system. Thus, a competition to decrement the size of the variables is carried out. Once again, no bit can be reduced without making the output noise go above the design constraint, which means that it is a minimal complying solution and the computation ends.

Table 5.3 shows a comparison on how the execution time is distributed between the different optimization stages in the min +1 algorithm and the incremental method. It is important to note that, even though the overall number of analysed WLVs is higher in the incremental method than in the classic approach, the total number of simulations is significantly smaller and thus the execution time is greatly reduced. It is also worth mentioning that the solution WLV found by both presented and reference algorithms was the same, but the incremental method reached it in a fraction of the time.

\subsection{Combined approach}

The techniques we have presented in sections 5.2 and 5.3 are both capable of achieving large accelerations with respect to other greedy approaches. But it is possible to combine both of them to obtain even further speed-ups. To do that, we introduce the idea of relaxing the confidence margins from the incremental method in the three-steps approach of the interpolative method:

1. The mUWL search is carried out with $N=10^{k_{1}}$ Monte-Carlo samples, where $k_{1}=k_{\text {ref }}-2$.

2. The mWS search, during which we fill the $M_{e}$ matrix, is done with $N=10^{k_{2}}$ Monte-Carlo samples, being $k_{2}=k_{r e f}-1$.

3. The exploration of the solution space, where we carry our simulations to adjust the estimations obtained from the values in the $M_{d}$ table, uses $N=10^{k_{r e f}}$ so the 
corrections and verification of the final results are accurate.

In this way, we are effectively capable of reducing the number of simulations carried out from two sides at once: Not only we avoid evaluating many word-length combinations through interpolation, but most of those we do are faster due to the reduced number of samples. And yet, since the final adjustment and verification of the results are done with the tightest confidence intervals, we can guarantee that the solution we obtain complies with the system constraints.

\subsection{Experimental results}

In this section we present the results obtained from applying the proposed approaches to a set of applications. We have used the variance $\left(\sigma^{2}\right)$ of the round-off noise as the metric for evaluating the word-length vectors, and the sum of word-lengths as the parameter to minimize (i.e. cost function). We use an upper bound of $\hat{p}=\Theta\left(\sigma^{2}\right)=10^{-6}$ as the noise constraint for all the tests. Thus, we set $N=10^{7}$ as the reference number of simulations to ensure results are accurate enough.

Also, as we described in Section 5.2.1, we need to set a value for the step size $b$ during the mWS stage of the interpolative search. We set $b=2$, the same value used in [SK95] for the experiments. We compare the results with our implementations of the classical min +1 algorithm and with the preplanned search, one of the fastest iterative methods.

In order to demonstrate the validity of the proposed methods, we have selected a set of meaningful applications to evaluate. First, we have applied the two optimization algorithms to two FIR filters with different sizes, the first one an order- 6 filter requiring 8 variables for its implementation, and the second one of order 29 with 56 variables involved in its design. Both of them are part of the design of a diplexer used to implement a TJ-II Infrared interferometer [Est11]. Here we use both linear filters to demonstrate how the speedups increase as the number of variables in the system grows. We also have evaluated a truncated Volterra filter of order $P=2$ and $L-1=2$ delay elements. This filter, as shown in [PM98], is an example of a non-linear system. Finally, we have considered one tap of a LMS adaptive filter from [SB04c] as an example of a system with feedback loops.

We show the execution times in Table 5.4. When implementing the min+1 algorithm, we have introduced a failsafe mechanism for local minima: If we detect that the sum of word-lengths in the word-length vector is larger than that of the smallest uniform wordlength vector that complies with the noise constraint, we will abort the search. We mark the corresponding simulation time with a ${ }^{f}$ in Table 5.4. In Figure 5.5 we show the speedups we achieve normalized with respect to the preplanned search.

As it can be observed, our algorithms are notably faster than the classical approaches. The performance of $m i n+1$ deteriorates rapidly as the size of the problem grows and the algorithm often falls into local minima, as with the FIR filter of order 29 after nearly 19 days of computation. The preplanned search offers better performance but it 
Table 5.4: Execution times for different benchmarks.

\begin{tabular}{|c|c|c|c|c|c|}
\hline Method & $\begin{array}{c}\text { 2-Order } \\
\text { IIR }\end{array}$ & LMS & $\begin{array}{c}\text { 6-Order } \\
\text { FIR }\end{array}$ & $\begin{array}{c}\text { Volterra } \\
\mathbf{2 x 2}\end{array}$ & $\begin{array}{c}\text { 29-Order } \\
\text { IIR }\end{array}$ \\
\hline \hline \# vars & 5 & 6 & 8 & 21 & 56 \\
\hline \hline Single iteration & $44 \mathrm{~s}$ & $64 \mathrm{~s} \mathrm{~s}$ & $72 \mathrm{~s}$ & $161 \mathrm{~s}$ & $496 \mathrm{~s}$ \\
\hline min+1 & $33^{\prime} 35^{\prime \prime} f$ & $38^{\prime} 28^{\prime \prime}$ & $9^{\prime} 14^{\prime \prime} f$ & $787^{\prime} 53^{\prime \prime}$ & $26511^{\prime} 12^{\prime \prime} f$ \\
\hline Preplanned & $21^{\prime} 35^{\prime \prime}$ & $38^{\prime} 42^{\prime \prime}$ & $52^{\prime} 44^{\prime \prime}$ & $176^{\prime} 8^{\prime \prime}$ & $4298^{\prime} 40^{\prime \prime}$ \\
\hline Interpolative (b=2) & $1^{\prime} 34^{\prime \prime}$ & $2^{\prime} 5^{\prime \prime}$ & $29^{\prime} 17^{\prime \prime}$ & $63^{\prime} 7^{\prime \prime}$ & $986^{\prime} 28^{\prime \prime}$ \\
\hline Interpolative (lim.) & $9^{\prime} 54^{\prime \prime}$ & $1^{\prime} 46^{\prime \prime}$ & $1^{\prime} 47^{\prime \prime}$ & $37^{\prime} 5 ”$ & $362^{\prime} 45^{\prime \prime}$ \\
\hline Incremental & $5^{\prime} 3^{\prime \prime}$ & $1^{\prime} 59^{\prime \prime}$ & $1^{\prime} 44^{\prime \prime}$ & $50^{\prime} 54^{\prime \prime}$ & $799^{\prime}$ \\
\hline Combined & $2^{\prime} 44^{\prime \prime}$ & $6^{\prime} 31^{\prime \prime}$ & $6^{\prime} 20^{\prime \prime}$ & $14^{\prime} 27^{\prime \prime}$ & $202^{\prime} 51^{\prime \prime}$ \\
\hline
\end{tabular}

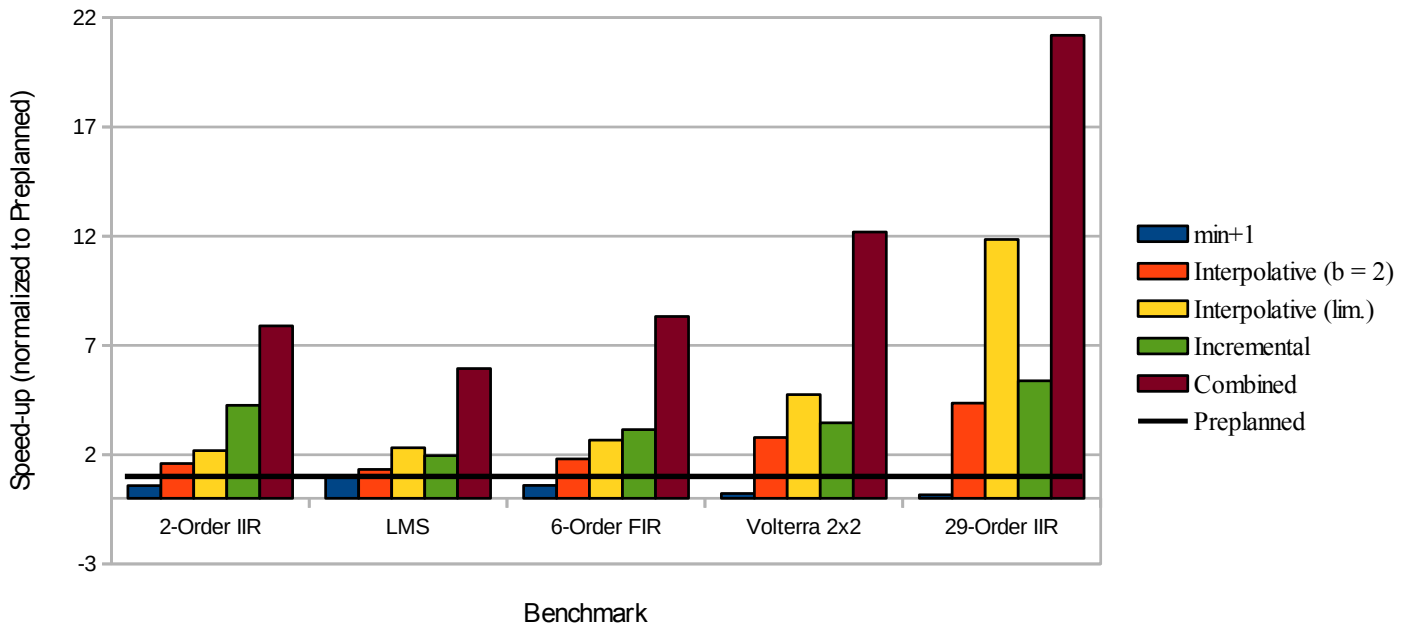

Fig. 5.5: Normalized speedups with respect to the preplanned search.

also shows a considerable degradation as the solution space grows. The execution times show a much more gentle increase for the interpolative and incremental methods. For small-sized problems like the FIR filter of order 6 our techniques offer speed-ups between $\mathrm{x} 1.79$ and $\mathrm{x} 5.93$, improvements that build up to between $\mathrm{x} 4.35$ and $\mathrm{x} 21.18$ for the largest systems being considered. When these results are compared with the min +1 algorithm, we can observe speed-ups up to x73.08 with the interpolative limit case, $\mathrm{x} 33.18$ with the incremental method, $\mathrm{x} 26$ with the interpolative method using $b=2$ and $\mathrm{x} 130.66$ with the combined approach. 


\subsection{Conclusions}

To minimize the amount of hardware used in a circuit based on fixed-point arithmetic it is necessary to explore solution spaces that grow exponentially with the size of the design. In this chapter we have started from the assumption that, with the current technological development, the ability to carry out that exploration rapidly prevails over the need of finding the optimal combination of word-lengths. Based on this, we have presented three methods that take advantage of different optimization opportunities to improve the overall execution time when using simulation-based approaches. We have shown how these methods are capable of achieving as good results as traditional greedy searches but in significantly reduced execution times. 


\section{Chapter 6}

\section{The HOPLITE framework}

This world of cold stone gives nothing in return to those who sleep while the restless burn. There are the few driven to flame, most are content to drown in the wake of dreams.

Neurosis,

Burn (The Eye of Every Storm)

As we saw in Chapter 2, word-length optimization draws together a wide variety of methodologies, metrics and optimization objectives, each of them relying on different implementations and dedicated toolchains. In the past years we have witnessed a rise in open-source modular frameworks that allow researchers and industrial partners to develop and test novel methodologies swiftly. Such have been the cases of compilers with LLVM [Lat02] and system architectures with gem5 $\left[\mathrm{BBB}^{+} 11\right]$. Given the variety of requisites and approaches that word-length optimization conveys, a fully independent, modular framework is beneficial and desirable, but up to now, there is no open source project with these characteristics.

In this chapter we introduce the design and implementation of HOPLITE, a modular automated word-length optimization tool that is meant to fill that void, giving designers plenty of flexibility to develop modelling and search policies that best suit their objectives.

The rest of this chapter is organized as follows: The general HOPLITE work flow is described in Section 6.1. Following, in Section 6.2, we detail the implementation decisions, modules and interfaces that conform the framework. We put it to practice in Section 6.3, where we go over an execution example step by step, and finally we discuss different possibilities for extending the framework with new modules in Section 6.4, where we also show a case of an extension being integrated in HOPLITE.

The source code of the HOPLITE framework is available at https://github.com/ eSedano/hoplite under a MIT free software license (MIT). 


\subsection{Work flow}

The work flow of the HOPLITE framework is divided in four main tasks: Input parsing, dynamic range and quantization noise modelling, search and output generation. We outline this flow in Figure 6.1.

First, once the flow reads the configuration files and the arguments fed by the user in the command line, the I/O interface module takes care of parsing the input code and, with that information, it generates the system graph that will be used through the rest of the process.

Then, the modelling engine is invoked. Here, designers can explore different methodologies for dynamic range estimation and RON evaluation. Depending on the type of signal and RON model, this engine might generate the equations to compute the output, create new structures ready for Monte-Carlo simulations or generate and compile code for running external modules on demand. It is possible to use partitioner modules at this stage to carry out hierarchical or compartmentalized analyses of the system. The processing made at this stage may be varied, but it all must be guided towards generating information that will be used by the optimization process to direct the exploration of the solution space.

The search engine starts after the computation in the modelling engine is finished. The actual optimization process takes place in this stage. Search modules send requests to the modelling module for different metrics of round-off noise and use them to decide the best combination of word-lengths for the system that satisfies the constraints imposed by the designer. The flexibility of the modular approach and the straightforward design of the system graph, which we discuss in Section 6.2.3, allows designers to devise, implement and evaluate all sorts of optimization algorithms easily.

Finally, once the search engine determines that it has found the desired complying solution, the I/O interface is called again to generate the output for the processing. This output can, again, be represented in any format the designers may require, from annotated compiler intermediate representation to source code files with the adequate data types.

We have designed the flow to be modular, reusable and easy to extend. With these goals in mind, we consider that the four-step approach we have described in this section grants a great degree of freedom for researchers and developers to implement all sorts of approaches to the complex problem of word-length optimization.

\subsection{Implementation}

The implementation of an automated modular framework entails many design decisions that depend mainly on the intended purpose of the application, the specifications, and the foresight and good criteria of its developers. In this section we detail key decisions we made during the development of HOPLITE. 


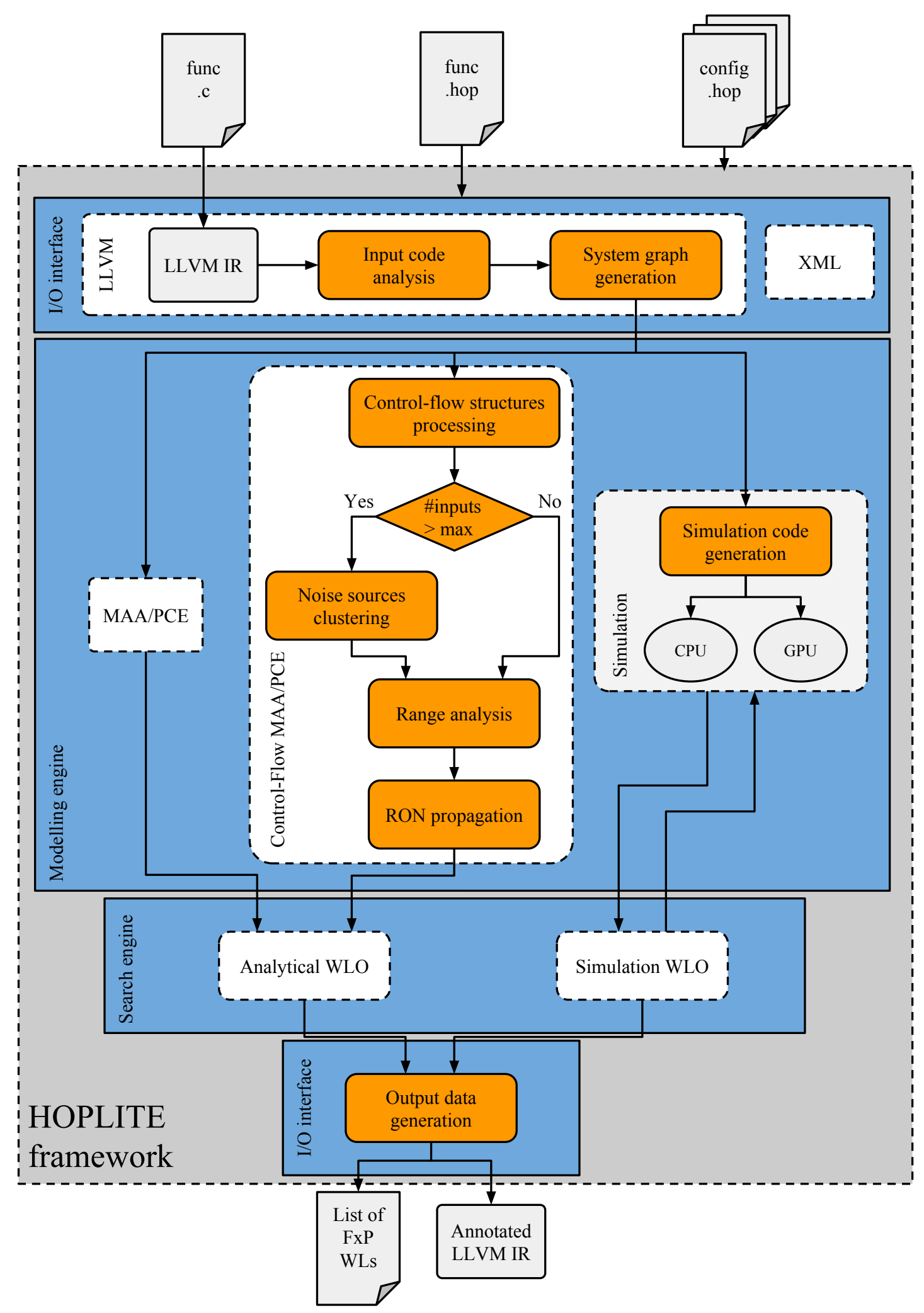

Fig. 6.1: HOPLITE framework work flow. 
Table 6.1: Language requisites and availability.

\begin{tabular}{|c|c|c|c|c|}
\hline Requisite & MATLAB & Octave & C/C++ & Python \\
\hline \hline Simple & No & No & Some & Yes \\
\hline Algebra \& symbolic systems & Yes & Some & Yes & Yes \\
\hline Memory management & Some & Some & No & Yes \\
\hline Support for external tools & Yes & Yes & Yes & Yes \\
\hline Free & No & Yes & Yes & Yes \\
\hline
\end{tabular}

\subsubsection{Programming language choice}

The decision of the programming language to use for implementing an application is never indisputable. There are many factors that developers have to take into account in order to choose among the hundreds of available languages: Suitability to what the code is intended to do, previous experience of the developer with the language, interaction of the program with the environment, long-term plans for the implementation, etc. Since these factors can be very subjective, there never is a unique answer.

While designing HOPLITE, we considered three different programming languages: $\mathrm{C} / \mathrm{C}++, \mathrm{MATLAB}$ and Python. The chosen language should have as many of these characteristics as possible:

- Language simplicity, offering a clean and straightforward syntax so code is easy to understand.

- An algebra system with symbolic computation support, given the nature of the analytical models to run within the framework.

- Efficient memory management, since we will usually work with large data sets and multiple replicated structures over time.

- A simple way for interacting with external tools and programs.

- Freely available.

A main argument in favour of using MATLAB would be that it is a language originally conceived for mathematical computation. It has its own algebra system, the Symbolic Math Toolbox, which deals efficiently with this type of systems. But in order to make the most of MATLAB it is necessary to use convoluted, far from readable syntax. When the code is written in a reader-friendly manner, nearly all the performance improvement disappears. Also, MATLAB is a proprietary and expensive tool. Octave, the free alternative to MATLAB, is not suitable either for the goals of the project. At the time of writing this document, its symbolic computation engine lacks important functionalities such as definite multiple integration. 
$\mathrm{C} / \mathrm{C}++$ is one of the most widespread and mature programming languages. It has been used in all types of applications and there are thousands of available extensions for the language. Among others, the LLVM compiler infrastructure, one of the I/O interfaces used by HOPLITE. It also has an algebra system with support for symbolic computation, called SymbolicC++ and the capability of calling external applications using system() or Popen(). But the memory management in $\mathrm{C}++$ is one of its major drawbacks. It lacks a garbage collector, so memory deallocation has to be treated explicitly, and tasks like deep copying complex data structures require careful and extensive coding.

For its part, Python meets all the requirements we list above. The syntax is simple while, at the same time, offers powerful constructs such as list comprehensions and native memory efficient hash tables (called dictionaries). The SciPy and SymPy packages offer a mature and robust environment for mathematical computation and operation with symbolic values. The fact that Python is a language mainly oriented to scripting makes the interaction with external programs a natural part of the intended applications of the language. This allows us to interact easily with tools like LLVM using subprocess.call() or subprocess.Popen(). Also, Python implements a garbage collector and has native support for deep copying data structures. Moreover, it offers extensive support for saving and restoring data structures to/from files as well as for multi-threaded programming.

Considering all of the above, also summarized in Table 6.1 , it is clear why we chose to use Python in HOPLITE.

\subsubsection{Configuration files}

Given the automated nature of HOPLITE, we need to define a way to feed information to the framework without continuously prompting the user to enter data. To that end, we define a set of configuration files from where the tool retrieves all the parameters it may need to function autonomously.

Configuration files in HOPLITE are written according to the YAML spec [BKEN09] and they have the .hop extension. One of the most basic configuration files is config.hop, used by the main execution flow to define the modules that will be used by default if none is passed as an argument in the command line call to the tool. Any additional parameter HOPLITE may need to work as a whole is included here, but the parameters in this configuration file are not visible by the modules.

Every function passed to the flow for its fixed-point optimization also needs to have its own configuration file. The base name of both source and configuration files must match, and in this case the latter will contain information relevant to the function that will be visible by all the modules. This information includes the symbolic names of the inputs to the function and the error constraints that must be observed while optimizing the design. Since systems with multiple outputs are considered, it is possible to define individual constraints for each output. The contents of this configuration are passed as a dictionary through the input_config argument in the constructor of every module. 
Finally, each module that extends HOPLITE has its own configuration file. In this case, the contents of these .hop files are completely module-dependent. We provide more information on these configuration files in Section 6.2.4.

\subsubsection{System graph representation}

The system graph is a key aspect of every word-length optimization engine. It is the internal representation of the design to optimize, and it must contain as much relevant information as it may be needed by the framework. An accurate description guarantees that the engine is capable of interpreting the characteristics of the design and that the decisions it will take will be well informed. Additionally, since the system graph will presumably be consulted constantly, efficient access to the data structure is a critical factor to take into account.

In HOPLITE we define the system graph as a dictionary where each entry contains relevant information about the design. The average access time to a Python dictionary is $O(1)$, while the amortized worst case is $O(n)$ [SJD $\left.{ }^{+} 12\right]$, thus achieving the efficiency we pursue. The system graph is defined as follows:

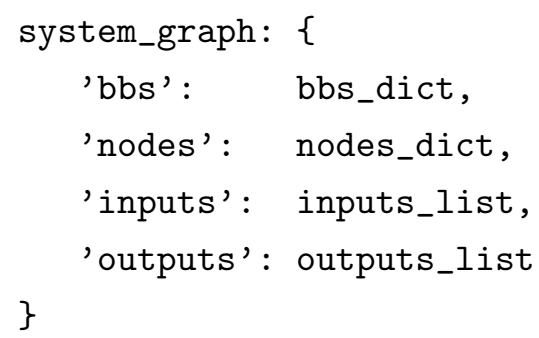

where bbs_dict is a dictionary of Basic Blocks (BB), nodes_dict a dictionary of all the nodes, and inputs_list and outputs_list are lists holding the IDs of the corresponding types of nodes.

We define a $\mathrm{BB}$ as the set of instructions encompassed between the destination of a branch and the following branch instruction, i.e. a maximized set of instructions that will always be executed unconditionally within a system graph. In HOPLITE, we consider $\mathrm{BB}$ with ID 0 an exception to this rule. BB 0 will be reserved for holding the inputs and outputs to the system exclusively. Consequently, a system will always have at least two BBs: one for the inputs and outputs (BB 0) and as many as needed for the instruction flow (BB 1 to $N$ ). Each entry of bbs_dict has the following structure:

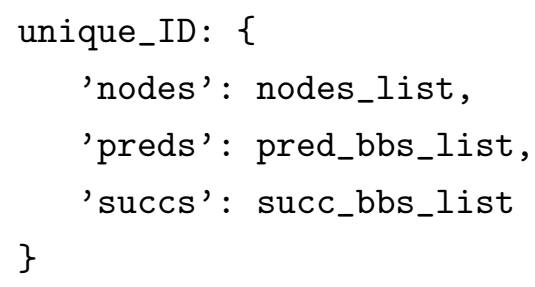

where unique_ID is a non-negative integer and the three fields of the dictionary are the ID lists containing the IDs of the nodes in the BB in nodes_list, of the predecessor 
BBs to the current one in pred_bbs_list and of the successor BBs in succ_bbs_list. Since access time to lists is $O(1)$ in both average and amortized worst cases, we do not incur in any performance penalty when accessing them.

In HOPLITE nodes represent either arithmetical or logical operations, inputs, outputs or control-flow blocks. The information about their contents and the relations among them is stored in nodes_dict. Each entry of this dictionary has the following structure:

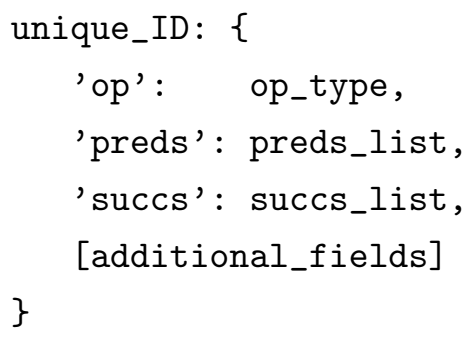

where unique_ID is a non-negative integer and, as in the case of the BBs, preds_list and succs_list are lists of the IDs of predecessor and successor nodes, respectively. In this case, the order of IDs in the preds_list is important, as it determines the order of the operands. The op_type is a string with the type of operator (add, sub, mul, etc.). Depending on the type of operator, we can see certain differences in the definition of the nodes. Regular arithmetic operations have no additional_fields defined, but some types of nodes do. For example, nodes with op_type const are constants, and they include an additional value field. Nodes of type cmp are comparators, and they have a cmp field with the type of comparison.

It must also be noted the special cases of br and phi nodes. The former are conditional branches and, instead of nodes, the IDs in their succs_list correspond with BB IDs. The latter type of node corresponds to PHI nodes like the ones we saw in Section 3.2.1. In their case, preds_list holds a list of tuples of two elements each, being the first one the ID of a predecessor node and the second one the ID of the BB that was executed right before the current one, so the PHI node is able to select a predecessor depending on the execution path that is being followed.

Finally, inputs_list and outputs_list are ordered lists with the IDs of the inputs and outputs to the system, respectively.

\subsubsection{Modules and interfaces}

HOPLITE is a highly modular framework so developers can change whatever step of the quantization process and introduce their own techniques. In order to maintain the stages uncoupled, we define a base class for each of them that specifies the public interfaces of the module, and only these interfaces should be used while doing intermodule communication.

Every module in HOPLITE must define a variable hoplite_unique_id and assign a value to it. The value of this variable will be a string with the same base name as the class implementing the module and the file where it is written. Also, the first thing 
every constructor has to do is to call the super constructor, which requires a parent argument:

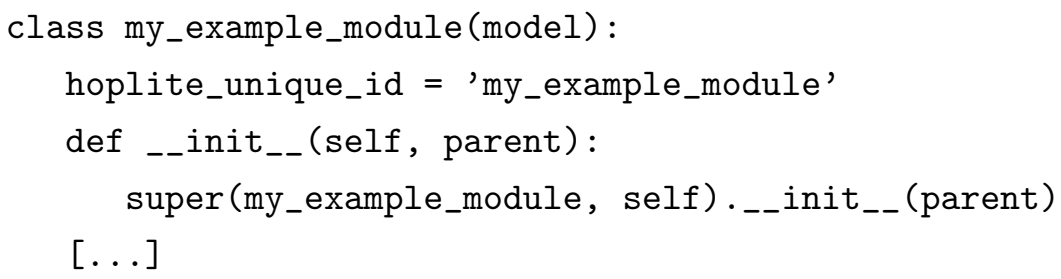

The parent argument gives the modules a reference pointer to the instance of the main hoplite object that instantiated them. Through this pointer, modules have visibility of the rest of instantiated components and they can call any function they require.

Additionally, every module will be joined by a .hop configuration file with the same base name as the module. This file will contain all the configuration parameters required exclusively by the module and that do not affect the rest of the work flow. The format of this file is the same one we defined in Section 6.2.2, and the values defined there will be available in a local config dictionary.

The rest of this section reviews the different modules available in HOPLITE and details the interface functions each of them provides.

\section{Input/Output}

The Input/Output module is responsible for generating the system graph (See Section 6.2.3), either reading the information from a file or using an external tool like LLVM. The I/O module also performs an initial set of transformations on the system graph, such as dead code elimination, static loop unrolling or operation transformations. Finally, this module offers a set of functions to consult the results of the optimization process.

I/O interfaces are stored in the io_interfaces sub-folder. The classes inherit from io_interface and they implement the following functions:

- get_graph() returns the final (i.e. after transformations) system graph that will be modelled and optimized. It will be called by the main HOPLITE engine during runtime.

- get_wl([var]) should be called only after the optimization process has finished. It returns the chosen word-length for the variable var, or a dictionary of all the variables IDs and their word-lengths if no argument is specified. The key and format of the associated word-lengths may depend on the tool (if any) HOPLITE is interfacing with. Thus, different I/O modules may accept different types of data as the var parameter. It can range from an integer indicating the appearance order of the variable in an input list to a memory pointer to an element in the Intermediate Representation of a compiler. The I/O interface is responsible for corresponding this format with the internal representation used by the framework. 
Any processing that the I/O interface needs to perform before the call to get_graph () must be done during the call to the constructor of the class. For example, the LLVM interface verifies that the compilation framework is installed, checks if the HOPLITE pass is present and, if not, copies the relevant files into the LLVM folder and compiles the pass.

\section{Signal and RON models}

One of the key steps in the word-length optimization process is modelling the effects of RON accurately. The signal and RON models are the part of HOPLITE that takes care of this step. Models work directly on the system graph and they can manipulate it as much as needed, but they must ensure that the I/O interface is capable of retrieving all the required data after the processing. Models also receive, optionally, a partitioner. We will cover partitioners in the next section.

Signal and RON models are stored in the models sub-folder. The classes inherit from the model class and they implement the following functions, where wlv, is a dictionary that uses the variable ID as key and its fractional word-length as value:

- compute () is the function called by the main engine during runtime to allow the model setting up everything and carrying as much calculations as required without a specific word-length configuration to evaluate. During this call, modules based on analytical models may operate using symbolical computation to generate expressions for the outputs of the system dependent on the word-lengths assigned to each variable.

- get_signal_mean([output]) returns the value of the signal mean for the requested output. If no parameter is passed to the function, it will return a dictionary with the signal means of all the outputs in the system, using the output node ID as key.

- get_signal_variance([output]) returns the value of the signal variance for the requested output. As in the previous case, if no parameter is passed to the function, it will return a dictionary with the signal variances of all the outputs in the system, using the output node ID as key.

- get_noise_mean (wlv, [output]) returns the value of the mean of the requested output when the variables have the word-lengths specified in wlv. If no parameter is passed to the function, it will return a dictionary with the means of all the outputs in the system, using the output node ID as key.

- get_noise_variance(wlv, [output]) returns the value of the variance of the requested output when the variables have the word-lengths specified in wlv. If no parameter is passed to the function, it will return a dictionary with the variances of all the outputs in the system, using the output node ID as key. 
- get_max_mismatch (wlv, [output]) returns the maximum mismatch value for the requested output when the variables have the word-lengths specified in wlv. If no parameter is passed to the function, it will return a dictionary with the maximum mismatches of all the outputs in the system, using the output node ID as key.

\section{Partitioners}

Partitioning the system graph to treat each sub-block independently is not a widespread technique at the time this Ph.D. Thesis is presented. But different works such as [PRMS10b] or the technique we propose in Section 3.4 give us enough grounds to consider this approach will become a common practice in the near future. Thus, HOPLITE gives the option to designers of supplying a partitioner to the signal and RON model.

Partitioners are, to a large extent, dependent on certain signal and RON models and it is expected that certain combinations of models and partitioners will not work well together. Designers and users must be aware of this when using HOPLITE. As every other module in HOPLITE, the partitioner module can be directly programmed in Python or be used as an interface with external tools specifically focused on partitioning such as METIS [KK98, Kar14].

Partitioners are stored in the partitioners sub-folder and the classes inherit from the partitioner class, implementing the following function:

- get_partitions(graph, nodes, parameters) is the function in charge of studying the system graph provided in graph and create groups from the indicated nodes according to the parameters defined for the task. The latter are defined per module and they can cover a variety of aspects such as stop and balance conditions, maximum number of nodes per partition or maximum number of levels in a hierarchical partition.

\section{Search algorithms}

Along with the signal and RON models, search algorithms are one of the cornerstones of the fixed point word-length optimization process and they have attracted the interest of many researchers over the years. They are responsible for selecting the best wordlength combinations to ensure efficient implementations that comply with the different constrains. During the search process, the modules in this category will consult regularly the signal and RON models for the mean and variance values given different combinations of word-lengths.

Since several works in the past have also dealt with the concept of dynamic range optimization, the responsibility of determining both integer and fractional lengths falls into the search algorithm modules.

Search algorithms are stored in the searches sub-folder and the classes inherit from the search class and implement the following functions: 


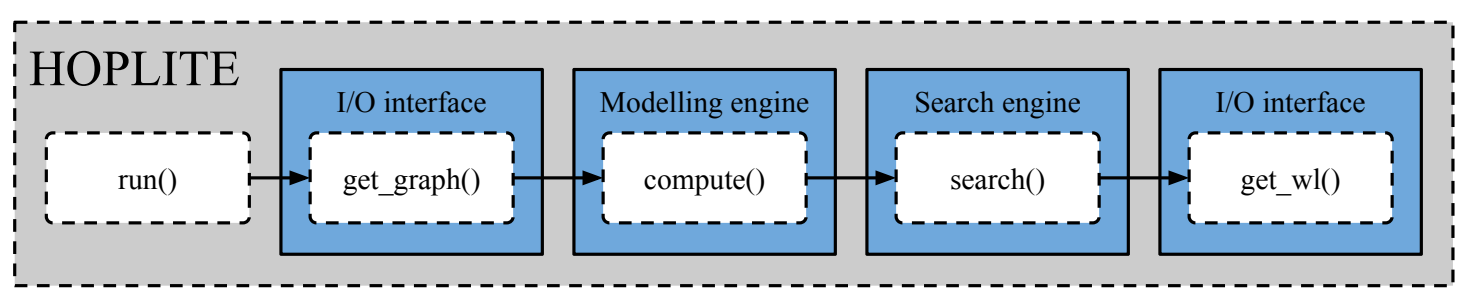

Fig. 6.2: HOPLITE call sequence.

- dynamic_range() does the analysis of the dynamic range of the variables in the system, determining the length of the integer part of each variable. If dynamic range optimization techniques are applied, the call to this function will trigger the optimization process.

- fractional_lenght( $)$ starts the exploration of the solution space to look for suitable combinations of fractional word-lengths, trying to optimize the fixed-point implementation of the design.

- search () is a function intended for those search algorithms in which determining dynamic ranges and fractional lengths cannot be split in two independent steps. When that happens, a flag in the module configuration file (single_search_call) must be enabled, and then HOPLITE will call this method instead of the two above.

- get_wl ( [var]) returns the chosen word-length for variable var during the optimization process. If no argument is specified it returns a dictionary of all the variables IDs as keys and their corresponding word-lengths as values. This function should be called only after the optimization process has finished. Here, var always refers to the ID of nodes in the HOPLITE system graph. The I/O interface is responsible for translating identifiers outside HOPLITE to the internal ID that is passed as argument here.

\subsubsection{Calling sequence}

The main sequence of function calls during the execution of HOPLITE, shown in Figure 6.2, is fixed. When the framework is invoked, and as we saw in Section 6.1, the first block that runs is the I/O interface. Specifically, through the get_graph() function. Then, the modelling engine is called through compute () so that the required equations or structures are set up. Once that unit has finished, the HOPLITE engine starts the proper word-length optimization process by calling the $\operatorname{search}()$ function from the search engine. Finally, depending on whether the framework is called as a standalone unit or as part of a larger environment, the get_wl() will be invoked in order to get the word-lengths of all the signals, or the framework will remain idle waiting for the larger environment to access it through both get_wl() and get_wl(var) functions. 
Some methodologies may not fit perfectly in this four-steps scheme. One of the advantages of HOPLITE is that the interfaces are general enough to allow designers to fit their procedures in the flow effortlessly. We will see an example of this in Section 6.4, where we will detail how to adapt an external tool for it to be called from within HOPLITE.

\subsection{Case study}

In this section we demonstrate the behaviour of HOPLITE by showing the steps and decisions it takes when we use it to optimize the fixed-point implementation of the following function

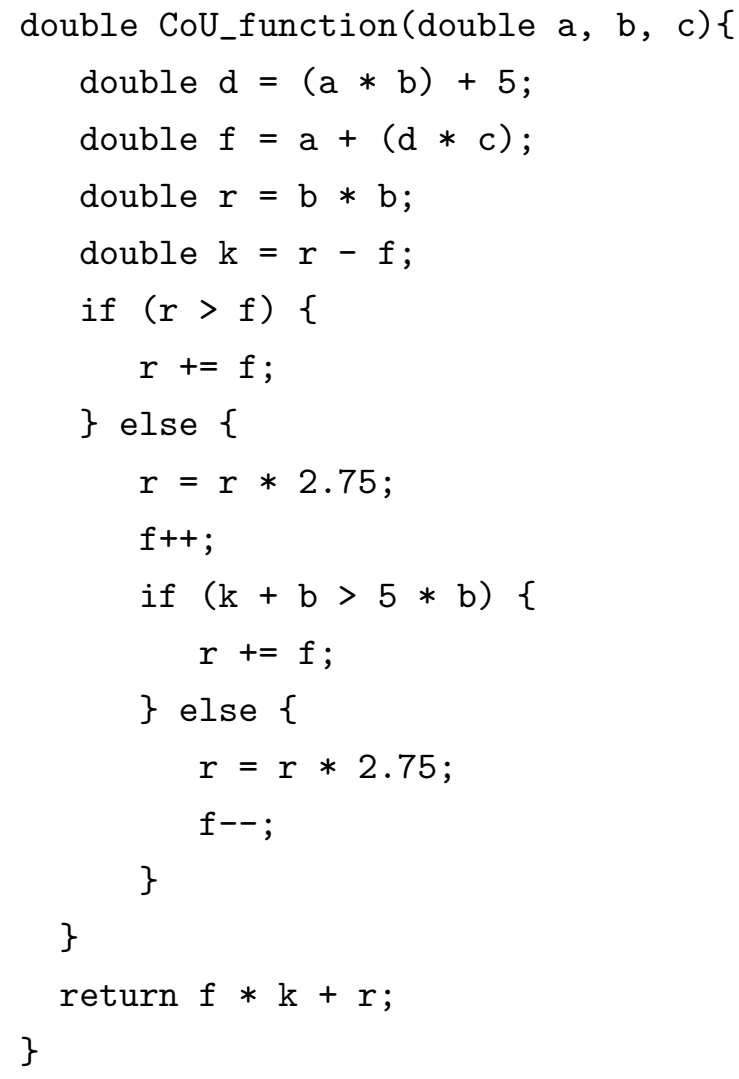

which we have defined in the file CoU_function.c along with CoU_function.hop, its configuration file, where we set the RON constraint for the only output in the code (the return statement) to a variance value of $10^{-5}$. We suppose these two files are stored in the folder ./eval. Then, to start the process we call the following command:

\$> hoplite ./eval/CoU_function.c -i llvm -m megpc -p hierfm -s max-1

In this call we are setting LLVM as the I/O interface and the MAA/PCE signal and RON model with support for conditional structures (Sections 3.2 and 3.3) and clustered noise injection (Section 3.4). The partitioner we have chosen to group the noise sources for the clustered noise injection is the hierarchical Fiduccia-Mattheyses we describe in Section 3.4.3, and finally we select a classic max-1 search algorithm. 


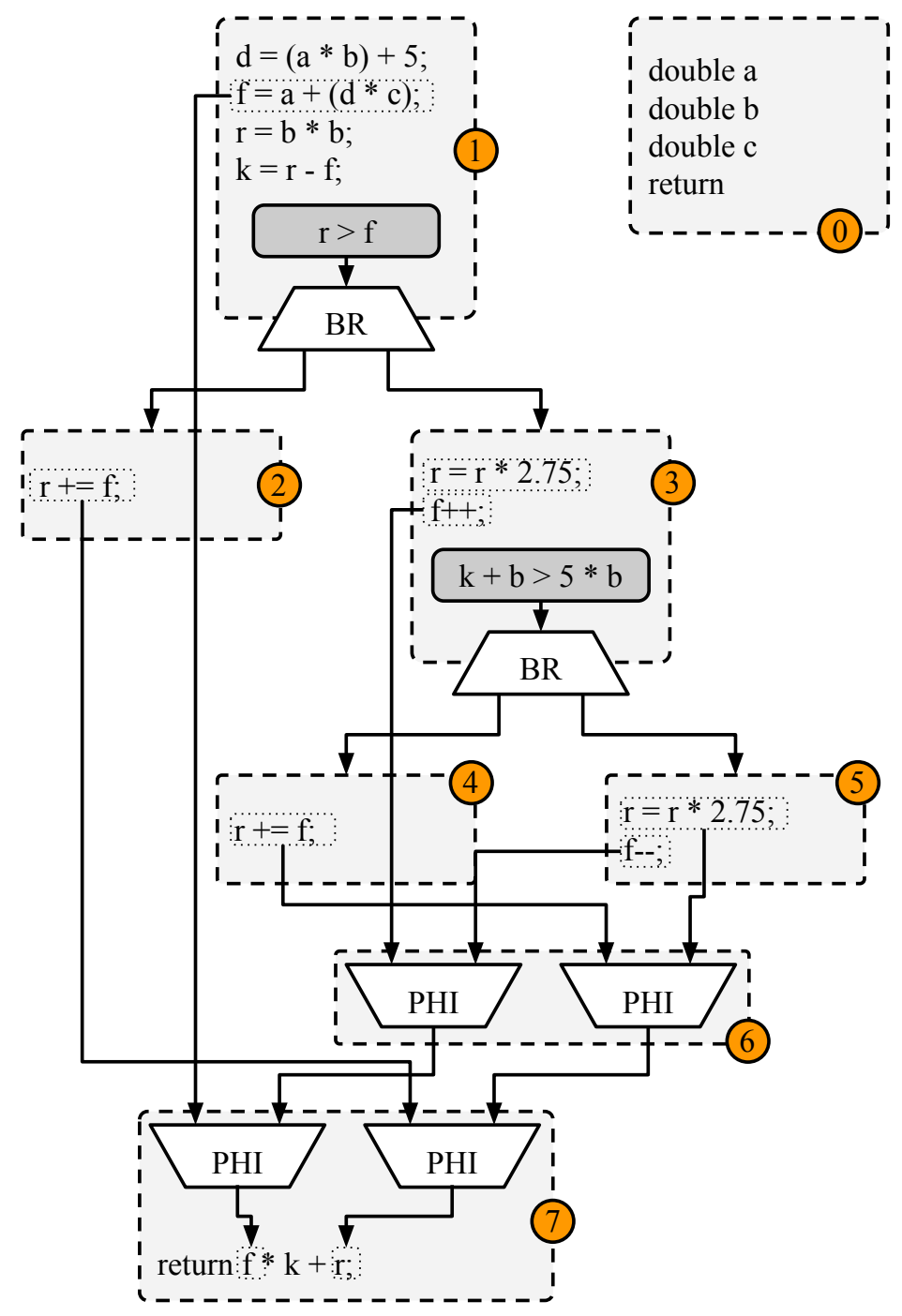

Fig. 6.3: Basic Blocks diagram of the case study.

The first thing HOPLITE does after checking that the environment is correctly set up is calling the get_graph () function of the I/O interface. This will compile CoU_function.c using Clang, the C language family front end of the LLVM framework, and generate CoU_function.bc, a LLVM bitcode file, on which we can then use the pass that parses the LLVM IR and extracts the system graph in HOPLITE format. Once the compiler generates the .bc file the interface calls said pass, reads the output and sets the system graph, which has 38 nodes divided into 8 BBs. The general structure is depicted in Figure 6.3

Then, the flow invokes the compute() function of the selected signal and RON model. The module first analyses the system graph and determines that, since all the control-flow structures in it are choice operators, it can directly generate the derivative system graphs. It will generate three of them: One for the case where $r>f$ (the first conditional) is true, and two for the case of the condition being false: one for each outcome of the comparison $\mathrm{k}+\mathrm{b}>5 * \mathrm{~b}$. The tool takes charge of decluttering the graphs, removing 
Path A

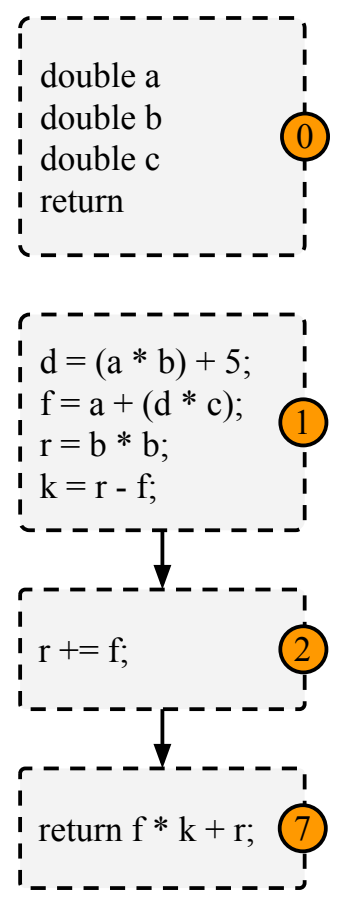

Path B
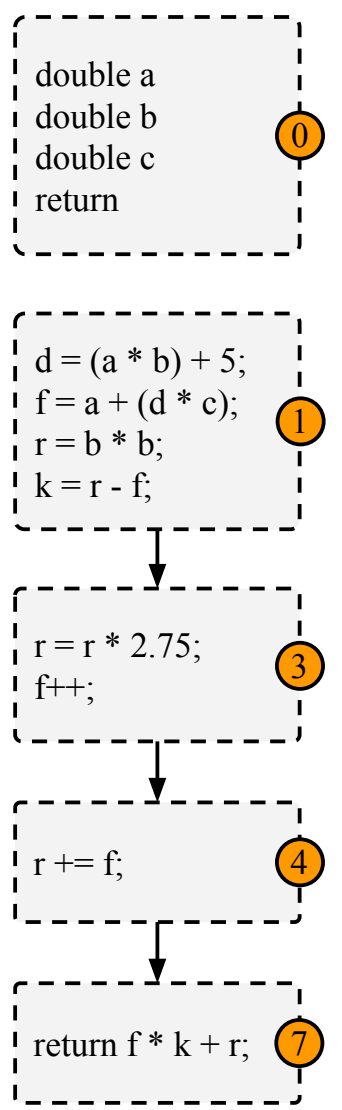

Path C

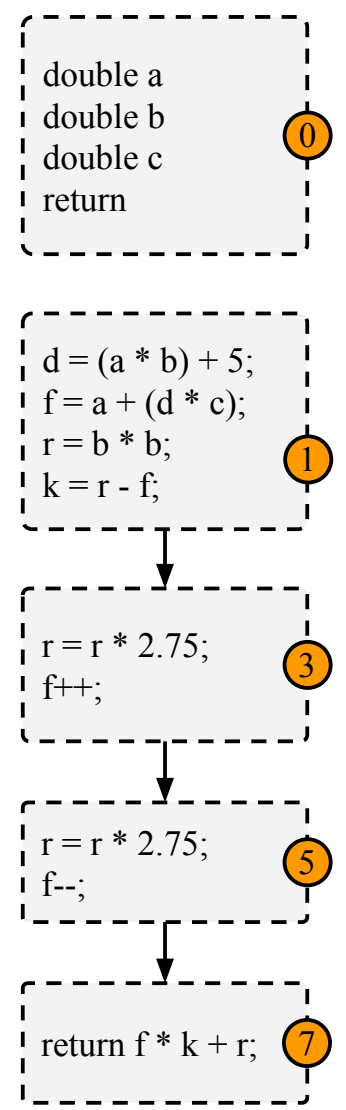

Fig. 6.4: Resulting execution paths from case study.

any trailing operation that is not part of the execution path any more. We show the three execution paths in Figure 6.4.

The modelling module starts then partitioning the input domain $[-1,1]^{3}$ following the procedure we presented in Section 3.2. Through the cond_pce.hop configuration file we have configured a $J_{\text {lim }}$ value of $10^{-2}$. With this precision, HOPLITE divides the domain in 288 partitions and, depending on the outcome of the conditionals for each of them, it assigns them to their corresponding derivative system graph.

After every partition is being linked to its system graph, HOPLITE will propagate the input PCE coefficients of each partition across the corresponding graph using MEgPC. Then, for each of the three graphs, it will inject the noise sources and repeat the process. Since we have told HOPLITE that we want to use the hierarchical FiducciaMattheyses partitioner, it is at this point where the module will execute. In order to compute the output PCE, the tool will inject the noises iteratively as we detailed in Section 3.4. For this demonstrator we set, through the hierfm.hop configuration file, a partition maximum size of 4 . This generates an average of 7 sets of noise sources per graph.

Once the propagation process is finished, we combine the partial results and we save 
the results. The results for signal mean and variance are numerical, while those for the RON are dependent on the word-lengths of the different inputs. These values will be accessed by the functions we detailed in Section 6.2 in order to provide the mean and variance values as requested.

Then, we move on to the optimization step, for which we use a classical max-1 greedy search. By consulting the get_noise_variance(wlv) interface of the model, it determines the best moves to do in the solution space exploration. Since the RON variance is saved in the signal and RON model as an equation depending exclusively on the fractional word-lengths, the result from the interface is almost immediate. This allows us to develop exhaustive searches capable of evaluating a large number of word-length configurations swiftly.

Finally, once the optimization process is complete, we store the word-length results thus making them available for the I/O interface.

Appendix B studies the execution time of the complete process, breaking it down into its basic components.

\subsection{Extending HOPLITE}

One of the most important requisites of the HOPLITE framework since its conception has been to provide support for the fast development of new RON estimation and wordlength optimization techniques. In this way, researchers and developers may only need to work on their module and test it within a fully working environment. As we pointed out in Section 6.2.1, this is one of the reasons to use Python as the programming language for the framework core.

The straightforward way of developing new modules that work in HOPLITE is to program them in native Python, interact with the surrounding blocks through the interfaces we have described in Section 6.2.4 and operate on the system graph directly. But some developers may not find Python suitable for the purpose of the module they wish to implement, or they might require additional infrastructure. The orientation towards scripting of Python will prove to be a powerful resource in this type of situation. It will be possible then, with minimal Python support, to interact with external applications independently of the language used to implement them. Such is the case of Phalanx [May14], a word-length optimization tool developed in the Departamento de Ingeniería Electrónica for a B.S. Thesis.

\subsubsection{The Phalanx case}

Phalanx is a tool to, given a system description, generate CUDA code for optimizing word-lengths using Monte-Carlo simulations and differential evolution and genetic algorithms specially designed for this purpose. The work follows the research line initiated by [CM12]. Even though it is developed to work with HOPLITE, it relies on a early 


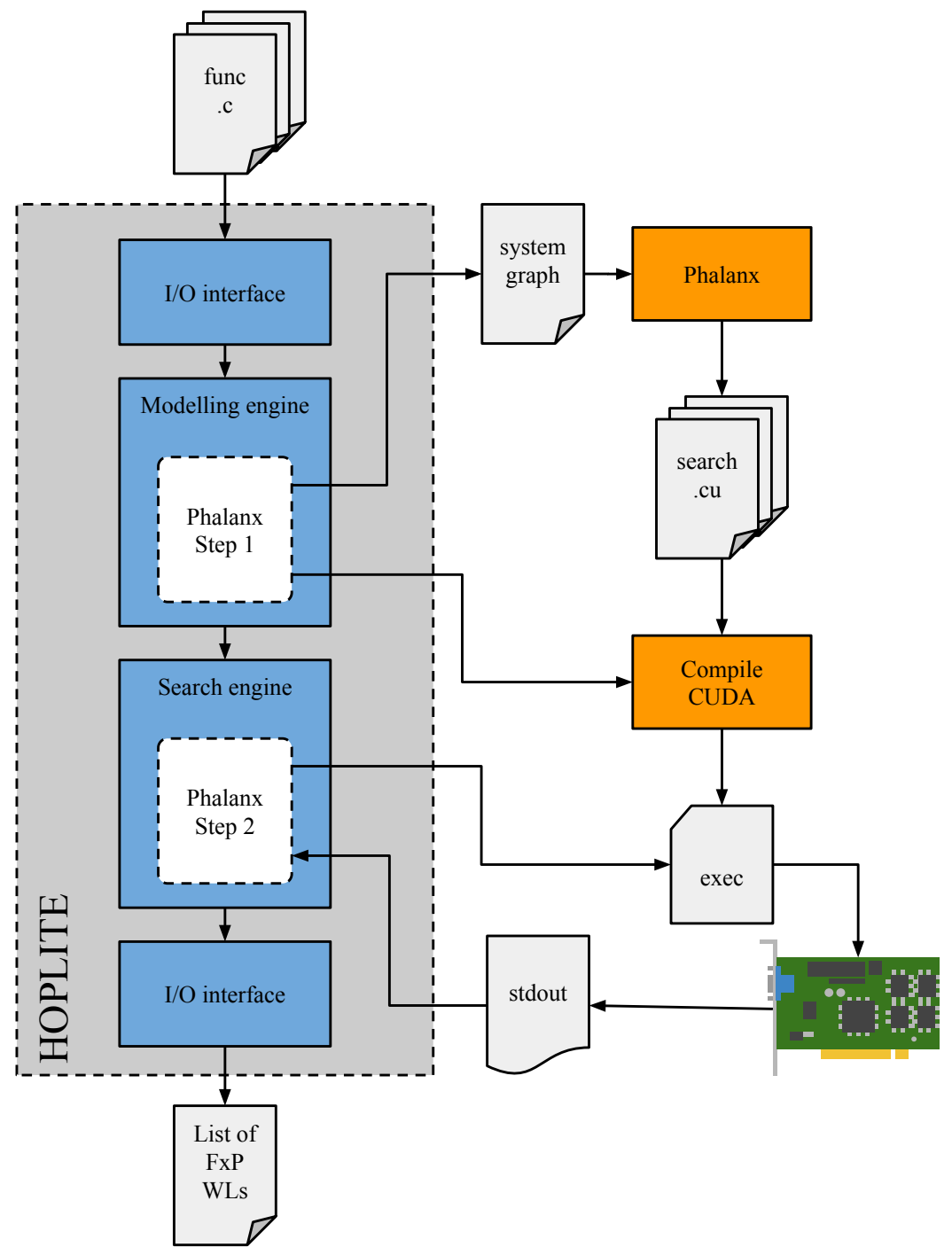

Fig. 6.5: Phalanx/HOPLITE integration flow.

iteration of the flow and it is programmed in $\mathrm{C}++$. The tool receives a system graph, traverses it and generates a number of files with all the infrastructure required to compile them and execute the word-length optimization on a Graphics Processing Unit (GPU).

In case we want to update Phalanx to the latest version of HOPLITE there would be no need for rewriting the whole tool. By adding minimal new code, the whole original source is completely reusable. Since the tool takes both modelling and search out of the CPU and they are absolutely dependent on each other, the whole flow can be divided among the two main blocks of the HOPLITE flow. We show the outline of this solution in Figure 6.5.

First, a signal and RON modelling module can process the system graph and generate an intermediate object, like a text file, with the description of the system. Then, using subprocess.call() we call the compiled Phalanx program that reads the file, builds the system graph and then does all the processing, resulting in the CUDA files for the word- 
length optimization. Moreover, we can then use the external command call to compile the CUDA sources into an executable program.

The second module would run during the search stage. Instead of calling the access functions of the model, using again subprocess.call(), we start the compiled CUDA word-length optimization capturing the stdout, where the final word-lengths will be dumped, and wait for it to finish. Then we only have to extract the results from the captured messages, waiting for the I/O module to request the information through the proper functions.

\subsection{Conclusions}

Currently available word-length optimization infrastructures are either not free ${ }^{1}$ or too strict in the way they implement the modelling and optimization methodologies. This factor holds up the development of new techniques, as designers have either restricted access to the tools or they need to spend ample time ensuring their code can work in synch with the rest of the environment.

In this chapter we have presented HOPLITE, a highly versatile, modular and open word-length optimization framework. It arises from the need of a platform with simple and clear interfaces that allows developers to implement and evaluate new techniques with minimal effort. We have introduced the work flow of the framework, explaining the different steps it comprises and its requisites. Also, we have detailed the internal structures shared across stages, reviewed the most important implementation decisions and described the interfaces of each flow stage, presenting a comprehensive view of the tool and its possibilities.

By using a scripting-oriented language like Python for implementing the core of HOPLITE, we give developers the freedom to use the language it suits their needs best, using Python exclusively to interface with the rest of the framework. In this chapter we have also seen an example of how this can be done by presenting the case of the Phalanx tool.

\footnotetext{
'Free' as in 'free speech', not as in 'free beer' [FSFI14]
} 


\title{
Chapter 7
}

\section{Conclusions and future work}

\author{
High in the North in a land called Svith- \\ jod there is a mountain. It is a hundred mi- \\ les long and a hundred miles high and once \\ every thousand years a little bird comes to \\ this mountain to sharpen its beak. When the \\ mountain has thus been worn away a single \\ day of eternity will have passed.
}

Hendrik Willem van Loon, The Story of Mankind

Fixed-point word-length optimization is a complex process with two main areas of interest: signal and noise modelling to quantify the effects of limited fractional precision on digital systems, and search algorithms to explore the solution space looking for the best combination of word-lengths. In this Ph.D. Thesis we have approached both topics and we have provided solutions for some of the most relevant issues that arise in practical situations.

In Chapter 3 we have proposed a methodology based on some of the ideas behind $\mathrm{ME}$-gPC for characterizing the dynamic range and round-off noise in non-linear systems with control-flow structures, improving greatly the applicability of this type of methodology as most of real-life system descriptions include these structures. This is a major breakthrough, as it is the first time that an analytical model is capable of estimating the RON in systems with general, control-flow structures such as if..then..else or while. .do statements. Our method approaches choice operators and loops structures uniformly, and it is consistent with MAA/PCE and ME-gPC. We have also addressed scalability, which is becoming one of the most pressing issues in models based on polynomial expansions. We have seen how the number of terms in the polynomials grows exponentially with the number of noise sources and we have proposed a novel approach, the clustered noise injection, to mitigate that effect. Also, we have seen how the different ways of grouping the RON sources to introduce can impact accuracy and we have 
presented a fast partitioning algorithm based on the classical Fiduccia-Mattheyses to minimize this impact. Finally, in order to compute the $\mathrm{C}$ matrix efficiently we have developed an optimized algorithm to avoid computing those values that we can infer from the beginning that will be zeroes. This algorithms is capable of computing large $\mathrm{C}$ matrices up to 1515 times faster than the direct method, while saving up to $99.9 \%$ of the required memory.

In order to prove the previous methods, in Chapter 4 we have presented a number of test cases that demonstrate the performance and accuracy of all of them. There, we have shown how our methodologies generate accurate representations in systems with controlflow structures and a large number of noise sources. The experimental results show how our methodologies are extremely accurate, showing deviations lower than $0.5 \%$ with respect to the reference MCS values in reasonable execution times. We have also explored how the trade-off between execution time and results accuracy, where by increasing the time allocated to the modelling task, the accuracy can increase up to $99.9 \%$ with respect to the reference values. Even though the evaluation through control-flow PCE/MAA is not negligible, the expressions obtained by the control-flow PCE/MAA can be reused, and consequently obtaining the noise metrics for a given WLV is almost immediate: This minimizes the time required to explore a solution space in search for an optimized solution to the WLO problem, making the approach perfectly suitable for accelerating modelling times.

The second major area of interest in word-length optimization, search algorithms, is addressed in Chapter 5. There, we have developed three novel greedy search algorithms that speed-up the Monte-Carlo based exploration of the solution space following two different approaches: First, the interpolative approach attempts to reduce the number of word-length configurations to evaluate by estimating some of the noise characterizations during the initial stages of the algorithm. Then it uses those values to guide the optimization, performing periodical evaluations to adjust the estimated results and to check that the solutions are valid. Second, the incremental approach reduces the execution time by allowing imprecise results, based on the widening of the confidence margins and consequent reduction of the number of evaluations each Monte-Carlo simulation performs. As the optimization process progresses, we tighten the confidence margins, focusing the last steps of the procedure towards the actual best solutions. Finally, we have combined the ideas of these other two algorithms into a single approach. The experimental results for a number of benchmarks show important improvements in computation times without evidencing degradation in the quality of the achieved solutions that range from $\times 1.79$ to $\times 130$ when compared to classical greedy approaches like min +1 or preplanned.

The final contribution of this Ph.D. Thesis is HOPLITE, an open source framework for word-length optimization where all the previous approaches are fully automated. We have introduced it in Chapter 6, where we explain all its characteristics, functioning, modules and interfaces. Word-length optimization is a wide area of research that encompasses very varied approaches, strategies, models and even objectives. Because of that, 
we wanted to create an environment that could be used in as many scenarios as possible. HOPLITE has a modular design, easy to extend and capable of interacting with external applications thanks to the scripting-oriented nature of its implementation language. We have presented a step by step application case to show the framework working, and we have explained how to extend HOPLITE with an external application written in a different programming language using Phalanx, a tool for generating fixed-point word-length optimization code for GPUs. Being fully automated, the required interaction with the designer is minimal, but since all the parameters can be adjusted from configuration files, it is still possible to have a high level of control over the conditions that drive the execution of the flow. Being published as open source code under a MIT license, designers and researchers from all around the globe can develop their own techniques to run on HOPLITE and contribute towards optimizing the existing code, positively influencing the whole community of users of the framework. This versatile framework will contribute in the reduction of the research and design cycle, as developers and professionals will find a reliable tool on which they can implement, test and verify new methodologies in a simple and efficient way.

\section{1. $\quad$ Future work}

In this Ph.D. Thesis we extended the MAA/PCE model to support control-flow structures, and we addressed the concerning issue of scalability. But even though the solutions we propose have allowed us to approach the problem considering today's requirements, we will soon be facing it again as the target applications continue to grow and their needs become more demanding. Scalability is always going to be a cause for concern when these methodologies are applied to extensive systems with a large number of inputs. In this Ph.D. Thesis we have presented the building blocks that will be the foundations of future methodologies when dealing with this issue. Through adequate partitioning and clustering of both signals and noises, and by relying on the support that modern parallel hardware can provide, we can address large systems methodically and still obtain accurate results.

Also, the methods and models that we have developed in this Ph.D. Thesis, as well as the body of work that serves as foundation for them (AA, MAA, MAA/PCE) are perfectly valid and feasible for several exciting lines of research: the quantization of custom, not IEEE-754 compliant floating-point operators, the use of block-floating-point arithmetic and the modelling and characterization of systems using mixed floating/fixed-point arithmetic. Tuning the methodologies we have presented to represent the particularities of these formats is a task that still requires further developments.

In any case, there are topics related to the methodologies we have presented here that still need to be addressed. For example, considering the topic of the precision of the decision operators not covered here, if only a tiny area of the input domain causes the decision to change direction, it may happen that the procedure we describe in Section 
3.2 does not identify it and thus decides that the whole domain takes always the same direction. Because of this, in order to improve accuracy, it would be very interesting to find a solution to this issue.

The noise clustering methodology we have presented also presents new lines of research. Our method is based on the original Fiduccia-Mattheyses algorithm, which was conceived for doing two-way partitioning. It would be interesting to investigate how to adapt some multi-way graph partitioning algorithms to our specific problem and evaluate their performance. Also, the type of heuristic we presented aims at keeping a low number of polynomial terms at any time, but in certain cases that may come at the cost of significant loss of accuracy. Other approaches based on keeping the highly coupled signals together regardless of the set size could achieve higher accuracy but impacting negatively on the execution time. It will be very interesting to investigate this trade-off in order to achieve the most accurate results in the shortest possible time.

On the other hand, optimization algorithms offer plenty of new opportunities. As analytical models are capable of representing more and more different types of systems, the requisite of minimizing the number of evaluations that Monte-Carlo based modelling imposes can become less relevant, and researchers are free to design new heuristics to guide their searches. The number of new optimization algorithms and methodologies keeps growing every day, and novel families of them, such as Harmonic Resonance algorithms or Cuckoo optimization algorithms have appeared recently to refine the work of previous evolutionary algorithms. Adapting these methodologies to the fractional word-length optimization domain will be a most interesting body of work.

Hopefully, as an open framework, HOPLITE will give researchers and developers around the world the necessary support to discover these new ways in the challenging field of word-length optimization and system modelling. HOPLITE is currently in an early stage of development and there is still work to do for it to become a reference point in its field. Some of the features that are still not supported by the framework but we already plan to implement are:

- Support for loop structures from Section 3.2.1 in the automated control-flow PCE modelling module.

- The possibility of setting fixed word-lengths before the processing starts.

- The capability of specifying subsystems within the whole system graph and optimizing them independently.

- New modelling modules for floating-point word-length optimization as well as mixed floating/fixed-point approaches.

- The implementation of grid-supported multi-threaded execution of the parallel parts of the control-flow PCE model and the extensions we have described in Section 3. 
The work in HOPLITE has only just begun. As more researchers join the development effort, the word-length optimization framework we have introduced in this Ph.D. Thesis will grow into a much more mature environment and it will evolve in ways we cannot imagine. It will be a most interesting exercise, in five years time, to look back and see all the progress HOPLITE will have done by then. Until then, we can only look forward to research new ways for extending and improving our tool. The road ahead will lead us through unknown places, but the trip will for sure be exciting. 


\title{
Appendix A
}

\section{Computation of the $\mathrm{C}$ matrix}

\author{
I'm not insane, sir. I have a finely calibrated \\ sense of acceptable risk.
}

\author{
John Scalzi, \\ Old Man's War
}

The C matrix is the tool that allows us to propagate PCE coefficients through nonlinear multiplications and divisions efficiently. As we saw in Section 3.1.3, the $\mathrm{C}$ matrix is a three-dimensional matrix of size $m \times m \times m$, being $m$ the length of the PCE base, where each position is defined as

$$
C(i, j, k)=\frac{<\psi_{i} \psi_{j} \psi_{k}>}{<\psi_{k}^{2}>} .
$$

Like PCE bases, a C matrix is defined by the number of input RVs (the dimension) and the maximum order of its polynomials. The names of the RVs are irrelevant for the scope of this discussion. For the sake of clarity, in the remaining of this section we will identify a $\mathrm{C}$ matrix of dimension $X$ and order $Y$ as $C_{d X o Y}$.

Useful as it is, one of the main problem it poses is that, as the size of the base grows, calculating the value of every position of the $\mathrm{C}$ matrix becomes an extremely time-consuming task, even if performed as a pre-processing stage. For example, a $C_{d 405}$ matrix, with base length 126 , has 2000376 entries, while a $C_{d 806}$, for which the base is 3003 elements long, will see that number increased up to 27081081027. Computing all those values iteratively, even once, would take an excessively long time. Also, storing them in a file would consume a vast amount of resources: With each value stored as a 32 -bit floating point number, the $C_{d 806}$ matrix would use almost $101 \mathrm{~GB}$ of memory.

In order to overcome these issues, we identify key properties of the $\mathrm{C}$ matrix and we take advantage of them to compute and store it fast and efficiently:

- The $\mathrm{C}$ matrix has symmetries. Given the commutative property of the multiplication, for every $i$ and $j$ it is always true that $C(i, j, k)=C(j, i, k)$. We can use 
this in our benefit to reduce the number of positions of the $\mathrm{C}$ matrix we need to calculate. Moreover, if we only consider the numerator of A.1, $\left\langle\psi_{i} \psi_{j} \psi_{k}\right\rangle$, we can see that all permutations of $i, j$ and $k$ are equivalent. We can thus minimize the number of integrals by computing the value of just one of the permutations and then using it for each of the six $\mathrm{C}$ matrix positions:

$$
\begin{gathered}
c_{i, j, k}=<\psi_{i} \psi_{j} \psi_{k}>, \\
C(i, j, k)=C(j, i, k)=\frac{c_{i, j, k}}{<\psi_{k}^{2}>}, \\
C(i, k, j)=C(k, i, j)=\frac{c_{i, j, k}}{<\psi_{j}^{2}>}, \\
C(j, k, i)=C(k, j, i)=\frac{c_{i, j, k}}{<\psi_{i}^{2}>} .
\end{gathered}
$$

- Even with the above reduction of computations, the number of combinations in $c_{i, j, k}$ can be considerably large. A $C_{d 405}$ matrix, with a base of length 126 , would still need to calculate 341376 expectancies. Even though this represents only a $17 \%$ of the total positions of the matrix, the process is still very time consuming. But since any given $\psi_{p}$ is a product of $d$ Legendre polynomials, one for each RV of the system $\phi_{1}, \cdots, \phi_{d}$, each of the polynomials of its corresponding order $p 1, \cdots, p d$, we can see how, being $P_{o}\left(\phi_{n}\right)$ the Legendre polynomial of order $o$ for the RV $\phi_{n}$,

$$
\psi_{p}=P_{p 1}\left(\phi_{1}\right) P_{p 2}\left(\phi_{2}\right) \cdots P_{p d}\left(\phi_{d}\right)
$$

so $c(i, j, k)$ can be expressed as

$$
\begin{aligned}
c(i, j, k)= & <P_{i 1}\left(\phi_{1}\right) \cdots P_{i d}\left(\phi_{d}\right) P_{j 1}\left(\phi_{1}\right) \cdots \\
& \cdots P_{j d}\left(\phi_{d}\right) P_{k 1}\left(\phi_{1}\right) \cdots P_{j d}\left(\phi_{d}\right)>.
\end{aligned}
$$

Taking advantage of the orthogonality of the Legendre polynomials, we can rewrite equation A.4 as

$$
c(i, j, k)=<P^{\phi_{1}}><P^{\phi_{2}}>\cdots<P^{\phi_{d}}>,
$$

where

$$
<P^{\phi_{n}}>=<P_{i n}\left(\phi_{n}\right) P_{j n}\left(\phi_{n}\right) P_{k n}\left(\phi_{n}\right)>,
$$

where again, applying the commutative property of the product, we just have to calculate $o \times(o-1) \times(o-2)$ expectancies.

- The $\mathrm{C}$ matrix is sparse. The number of non-zero values in a $C_{d 303}$ matrix is 382 out of 8000 elements (4.775\%), and it drops as the size of the matrix grows: $0.6 \%$ of the elements in a $C_{d 504}$ matrix are non-zero, while only the $0.09 \%$ (121275 out of 121287375 ) of values are non-zero in a $C_{d 804}$. We can take advantage of this in two ways. First, the memory requirements reduce drastically if we store the $\mathrm{C}$ matrix in a sparse format. Second, if we can detect zero values before applying A.1, we 
can minimize the number of times we execute integrals, which are time-consuming operations, thus reducing the execution time.

Two properties of the $\mathrm{C}$ matrix help us to identify zero values in the matrix early:

- Given any of the dimensions of the system, if the sum of the maximum polynomial order of that dimension in $\psi_{i}, \psi_{j}$ and $\psi_{k}$ is odd, it is asserted that $C(i, j, k)=0$. Thus, we can directly avoid computing any position with an odd sum of polynomial orders, knowing the result will be zero.

- If only one of $\psi_{i}, \psi_{j}$ and $\psi_{k}$ contributes with a non-zero value to the sum of maximum polynomial orders for a given dimension, it also holds true that $C(i, j, k)=0$. We can as well skip those elements from the computation.

Considering all of the above, we can compute and store the $\mathrm{C}$ matrix efficiently following Algorithm 3. Table A.1 compares the execution time of the proposed algorithm and the direct calculation of several $\mathrm{C}$ matrices for different input parameters. The results show large speed-ups when computing the matrix. The table also includes the percentage of non-zero values of each matrix to show that using a sparse structure for storing the $\mathrm{C}$ matrix leads to considerable memory savings.

When comparing the non-zero values of several $\mathrm{C}$ matrices calculated with both the direct and the proposed approaches we have observed a numerical difference between the two methods. This computational divergence is, in the worst case, in the order of $10^{-16}$. It is arguably introduced by floating-point imprecisions and the integration methods used by numerical engines. Being such a small value and given its source, we consider this divergences negligible.

The matter of efficiently inverting the A matrix from Table. 3.3 in order to propagate the PCE coefficients through a non-linear division is not considered in this Ph.D. Thesis, as this issue has already been addressed at large by other authors [Li09]. 


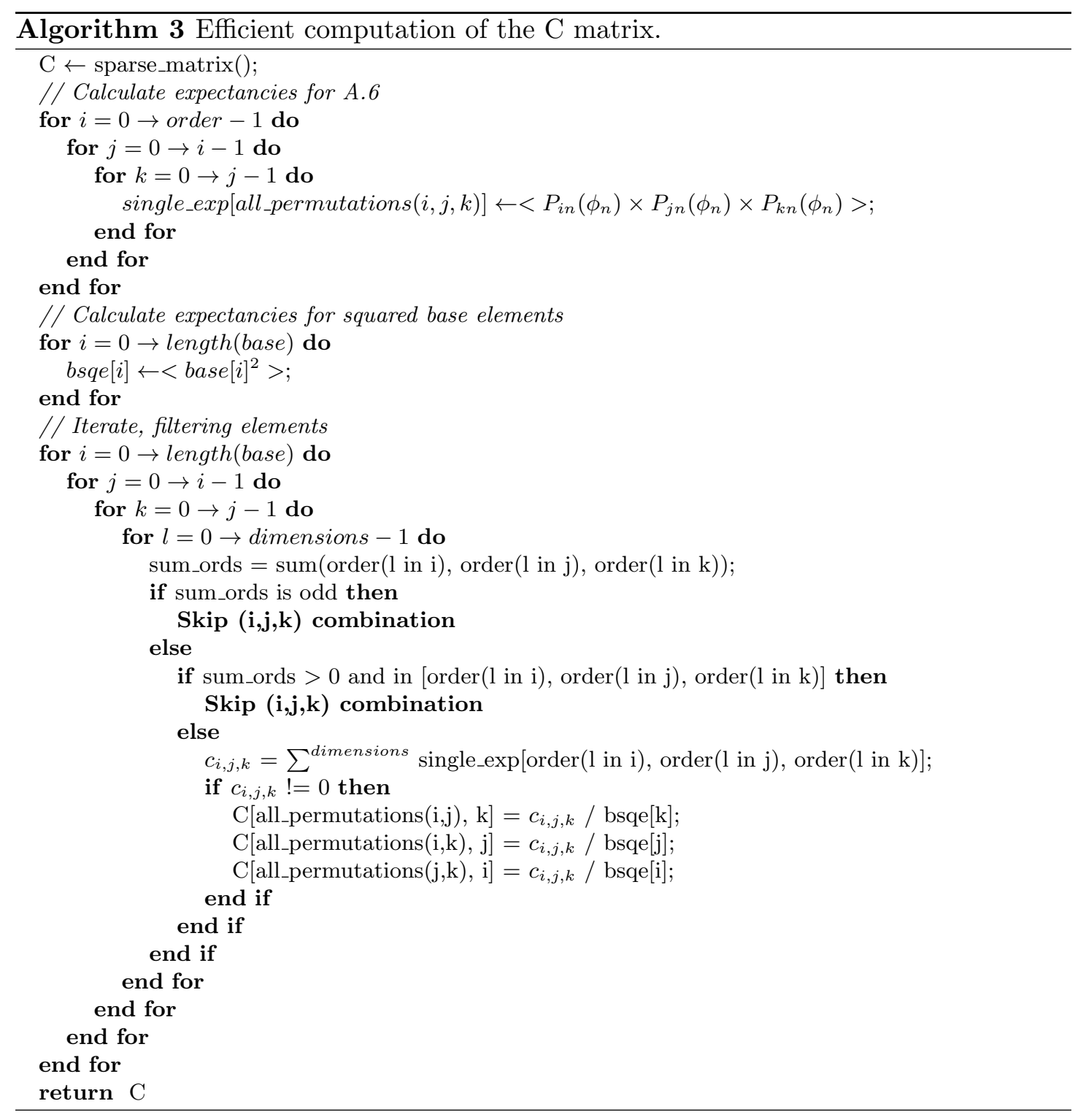

Table A.1: Comparison of methods for C matrix computation.

\begin{tabular}{|c|c||c|c||c|c|c|}
\hline \multicolumn{2}{|c||}{ Parameters } & \multicolumn{2}{c||}{ Elements in C } & \multicolumn{2}{c|}{ Execution time } & \multirow{2}{*}{ Speed-up } \\
\cline { 1 - 4 } Dim. & Order & Total & Non-zero & Direct & Proposed & \\
\hline \hline 3 & 2 & 1000 & $8.2 \%$ & $7.5 \mathrm{~s}$ & $0.8 \mathrm{~s}$ & $\times 9$ \\
\hline 3 & 3 & 8000 & $4.775 \%$ & $1 \mathrm{~m} 10 \mathrm{~s}$ & $1.8 \mathrm{~s}$ & $\times 39$ \\
\hline 3 & 4 & 42875 & $3.556 \%$ & $9 \mathrm{~m} 2 \mathrm{~s}$ & $4.5 \mathrm{~s}$ & $\times 120$ \\
\hline 6 & 2 & 21952 & $1.849 \%$ & 351 & $2.7 \mathrm{~s}$ & $\times 85$ \\
\hline 6 & 3 & 592704 & $0.6 \%$ & $113 \mathrm{~m} 48 \mathrm{~s}$ & $26.8 \mathrm{~s}$ & $\times 254$ \\
\hline 6 & 4 & 9261000 & $0.33 \%$ & $2222 \mathrm{~m} 46 \mathrm{~s}$ & $1 \mathrm{~m} 28 \mathrm{~s}$ & $\times 1515$ \\
\hline 12 & 2 & 753571 & $0.32 \%$ & $180 \mathrm{~m} 52 \mathrm{~s}$ & $51.7 \mathrm{~s}$ & $\times 209$ \\
\hline 12 & 3 & 94196375 & $0.04 \%$ & $20942 \mathrm{~m} 8 \mathrm{~s}$ & $28 \mathrm{~m} 30 \mathrm{~s}$ & $\times 734$ \\
\hline 12 & 4 & 6028568000 & $0.01 \%$ & $1446940 \mathrm{~m} 42 \mathrm{~s}$ & $963 \mathrm{~m}$ & $\times 1502$ \\
\hline
\end{tabular}




\section{Appendix B}

\section{Execution times in HOPLITE}

Wenn ist das Nunstück git und Slotermeyer? Ja! Beiherhund das Oder die Flipperwaldt gersput!

Ernest Scribbler

The Funniest Joke in the World

In Section 6.3 we have presented a case study where HOPLITE processed a function with control-flow structures and determined an optimized WLV for the resulting fixedpoint implementation. In this appendix we take a closer look at the execution time of the different steps involved in the optimisation of the CoU_function.

The execution times we show here have been obtained on a computer with an Intel ${ }^{\circledR}$ Core $^{\mathrm{TM}} \mathrm{i} 5-2557 \mathrm{M}$ CPU @ $1.70 \mathrm{GHz}$ and $4 \mathrm{~GB}$ of RAM. The control-flow PCE/MAA module, which takes most of the execution time, is a preliminary, single-threaded implementation of the methodologies we describe in Chapter 3. As such, costly operations that increase the overall execution time are often repeated and the multiple parallelization opportunities are not taken advantage of. This leaves plenty of room for further refinement and acceleration through code optimisation and a parallel implementation capable of making the most of multi-core architectures and clusters.

Table B.1 breaks down the complete process, detailing the steps it entails, the number of times each of them is carried out, and the execution time per step (both per iteration and in total). The modelling stage, being the most time-consuming, is further divided into its smaller processing parts. When a given step is repeated several times, the execution time per iteration reported in Table B.1 is an average value.

We can see how the partitioning at each choice operation happens only once: Computing the sub-domains that correspond to one of the paths of the control-flow structure automatically provides the sub-domains for the opposite one, thus avoiding unnecessary operations.

We also can observe here one of the main advantages of control-flow PCE/MAA with respect to simulation-based techniques: Once we compute the expressions of noise power 
Table B.1: Steps and execution time of HOPLITE for the CoU_function case study.

\begin{tabular}{|c|c|c|c|c|}
\hline \multirow{2}{*}{ Stage } & \multirow{2}{*}{ Processing step } & \multirow{2}{*}{ Repeats } & \multicolumn{2}{|c|}{ Exec. time } \\
\hline & & & Iter. & Total \\
\hline I/O interface & Load system graph (SG) & 1 & $0.2 \mathrm{~s}$ & $0.2 \mathrm{~s}$ \\
\hline \multirow{10}{*}{ Modelling } & Generate derivative SGs & 3 & $0.01 \mathrm{~s}$ & $0.03 \mathrm{~s}$ \\
\hline & Partition $k+b>5 * b$ & 1 & $123.4 \mathrm{~s}$ & $123.4 \mathrm{~s}$ \\
\hline & Propagate generic affine forms (Path C) & 1 & $9.8 \mathrm{~s}$ & $9.8 \mathrm{~s}$ \\
\hline & Replace generic affine forms (Path C) & 87 & $0.9 \mathrm{~s}$ & $78.3 \mathrm{~s}$ \\
\hline & Propagate generic affine forms (Path B) & 1 & $8.1 \mathrm{~s}$ & $8.1 \mathrm{~s}$ \\
\hline & Replace generic affine forms (Path B) & 33 & $0.85 \mathrm{~s}$ & $28.1 \mathrm{~s}$ \\
\hline & Partition $r>f$ & 1 & $21.7 \mathrm{~s}$ & $21.7 \mathrm{~s}$ \\
\hline & Propagate generic affine forms (Path A) & 1 & $8.1 \mathrm{~s}$ & $8.1 \mathrm{~s}$ \\
\hline & Replace generic affine forms (Path A) & 12 & $0.85 \mathrm{~s}$ & $9.6 \mathrm{~s}$ \\
\hline & Merge partial results & 1 & $2.9 \mathrm{~s}$ & $2.9 \mathrm{~s}$ \\
\hline \multirow{2}{*}{ Search } & Compute noise power for a given WLV & 324 & $0.4 \mathrm{~s}$ & $129.6 \mathrm{~s}$ \\
\hline & Select next best WLV & 35 & $0.01 \mathrm{~s}$ & $0.35 \mathrm{~s}$ \\
\hline \multirow[t]{2}{*}{$\mathrm{I} / \mathrm{O}$ interface } & Return results & 1 & $0.01 \mathrm{~s}$ & $0.01 \mathrm{~s}$ \\
\hline & Total execution time & & \multicolumn{2}{|c|}{$419.3 \mathrm{~s}$} \\
\hline
\end{tabular}

as a function of the fractional word-lengths of the variables in the system, obtaining the values for a given WLV is nearly immediate. Relying on simulation means that a new and time-consuming MC simulation with large sets of input samples has to be carried out to obtain the results. In Table B.1 we see that a new WLV is selected 35 times. The number of times that the noise has to be computed for different WLVs corresponds to those 35 selections times 9 variables to optimize, plus 9 extra computations in the last iteration when the algorithm determines that there is no better WLV to choose, and the search finishes.

Finally, we can observe how some of the steps in the modelling stage have a large number of iterations. As we have discussed previously in this Ph.D. Thesis, each of those iterations is completely independent from the others. Also, the partitioning step is a process where independent analysis of non-overlapping regions of the domain are considered. Thus, parallelizing different stages of the procedure could lead to large execution time savings. This is a feature that cannot be found in almost any other approach in the related work literature, as most of them rely on sequential treatment of the system and its characteristics. 
Overall, the execution time of the algorithm is good: we can see how the propagation of the affine forms is fast and it is in line with the execution times reported in [Est11]. Then, obtaining the partial result for a given sub-domain is done in less than a second, the process becoming time-demanding just because of the number of times it has to be repeated. Still, the partitioning of the domains could be optimised further. The order in which we study each conditional is not optimal: Each set of decisions is analysed independently, without sharing information between them. For example, to calculate the sub-domains mapped to the execution paths derived from cond_1 $=k+b>5 * b$, we first determine the sub-domains that map to the False path in cond__0 $=r>f$ and then we proceed to partition those with respect to cond_1. But if we first partitioned cond_0 we could reuse the results in both analysis.

The current preliminary implementation of of the proposed techniques was done with economy of code in mind. But it is clear that more intricate implementations will lead to significant speed-ups. Our initial estimations indicate that, by optimising the partitioning process and parallelizing the replacement of generic affine forms, we could reduce the total execution time of the algorithm by half. 


\section{Bibliography}

[AK95] Charles J. Alpert and Andrew B. Kahng. Recent directions in netlist partitioning: a survey. Integration, the VLSI Journal, 19(1-2):1-81, August 1995.

[AZ06] Arash Ahmadi and Mark Zwolinski. Word-length oriented multiobjective optimization of area and power consumption in dsp algorithm implementation. In Microelectronics, 2006 25th International Conference on, pages 614-617. IEEE, 2006.

[AZ07] Arash Ahmadi and Mark Zwolinski. A symbolic noise analysis approach to word-length optimization in dsp hardware. In Integrated Circuits, $200 \%$. ISIC'07. International Symposium on, pages 457-460. IEEE, 2007.

[AZ08] Arash Ahmadi and Mark Zwolinski. Symbolic noise analysis approach to computational hardware optimization. In Proceedings of the 45th annual Design Automation Conference, pages 391-396. ACM, 2008.

$\left[\mathrm{BBB}^{+} 11\right]$ Nathan Binkert, Bradford Beckmann, Gabriel Black, Steven K Reinhardt, Ali Saidi, Arkaprava Basu, Joel Hestness, Derek R Hower, Tushar Krishna, Somayeh Sardashti, et al. The gem5 simulator. ACM SIGARCH Computer Architecture News, 39(2):1-7, 2011.

[BC12] David Boland and George A Constantinides. A scalable approach for automated precision analysis. In Proceedings of the ACM/SIGDA international symposium on Field Programmable Gate Arrays, pages 185-194. ACM, 2012 .

[BC13] David Boland and George A Constantinides. A scalable precision analysis framework. Multimedia, IEEE Transactions on, 15(2):242-256, 2013.

[BCC05] C-S Bouganis, George A Constantinides, and Peter YK Cheung. A novel 2d filter design methodology for heterogeneous devices. In Field-Programmable Custom Computing Machines, 2005. FCCM 2005. 13th Annual IEEE Symposium on, pages 13-22. IEEE, 2005. 
[BCL $\left.{ }^{+} 14\right]$ Pablo Barrio, Carlos Carreras, Juan A López, Óscar Robles, Ruzica Jevtic, and Roberto Sierra. Memory optimization in fpga-accelerated scientific codes based on unstructured meshes. Journal of Systems Architecture, 60(7):579-591, 2014.

[BCMM11] Andrei Banciu, Emmanuel Casseau, Daniel Menard, and Thierry Michel. Stochastic modeling for floating-point to fixed-point conversion. In Signal Processing Systems (SiPS), 2011 IEEE Workshop on, pages 180-185. IEEE, 2011.

[Ber76] Claude Berge. Graphs and Hypergraphs. Elsevier, 1976.

[BKEN09] Oren Ben-Kiki, Clark Evans, and Ingy döt Net. YAML Ain't Markup Language $\left(Y_{A M L}{ }^{\mathrm{TM}}\right.$ ) version 1.2. http://www.yaml.org/spec/1.2/spec. html, October 2009.

[BPCC09] Christos-S Bouganis, Sung-Boem Park, George A Constantinides, and Peter YK Cheung. Synthesis and optimization of $2 d$ filter designs for heterogeneous fpgas. ACM Transactions on Reconfigurable Technology and Systems (TRETS), 1(4):24, 2009.

[BR04] P Belanovic and M Rupp. Fixify: a toolset for automated floating-point to fixed-point conversion. In International Conference on Computing, Communications and Control Technologies (CCCT'04), pages 28-32. Citeseer, 2004.

[BR05] Pavle Belanovic and Markus Rupp. Automated floating-point to fixedpoint conversion with the fixify environment. In Rapid System Prototyping, 2005.(RSP 2005). The 16th IEEE International Workshop on, pages $172-$ 178. IEEE, 2005.

[BSB07] Géraud Blatman, Bruno Sudret, and Marc Berveiller. Quasi random numbers in stochastic finite element analysis. Mécanique $\mathcal{E}$ Industries, 8(3):289297, August 2007.

[Caf08] Gabriel Caffarena. Combined Word-Length Allocation and High-Level Synthesis of Digital Signald Processing Circuits. PhD thesis, 2008.

[CB94] H Choi and WP Burleson. Search-based wordlength optimization for vlsi/dsp synthesis. In VLSI Signal Processing, VII, 1994.,[Workshop on], pages 198-207. IEEE, 1994.

[CC10a] Gabriel Caffarena and Carlos Carreras. Architectural synthesis of dsp circuits under simultaneous error and time constraints. In VLSI System on Chip Conference (VLSI-SoC), 2010 18th IEEE/IFIP, pages 322-327. IEEE, 2010 . 
[CC10b] Gabriel Caffarena and Carlos Carreras. Precision-wise architectural synthesis of dsp circuits. In 18th European Signal Processing Conference (EUSIPCO-2010), pages 562-566. EURASIP, 2010.

$\left[\mathrm{CCC}^{+} 06\right]$ Gabriel Caffarena, George A Constantinides, Peter YK Cheung, Carlos Carreras, and Octavio Nieto-Taladriz. Optimal combined word-length allocation and architectural synthesis of digital signal processing circuits. Circuits and Systems II: Express Briefs, IEEE Transactions on, 53(5):339-343, 2006.

[CCC09] Jonathan A Clarke, George A Constantinides, and Peter YK Cheung. Wordlength selection for power minimization via nonlinear optimization. $A C M$ Transactions on Design Automation of Electronic Systems (TODAES), 14(3):39, 2009.

[CCL99] George A Constantinides, Peter YK Cheung, and Wayne Luk. Truncation noise in fixed-point sfgs [digital filters]. Electronics Letters, 35(23):20122014, 1999 .

[CCL00] George A Constantinides, Peter YK Cheung, and Wayne Luk. Multiple precision for resource minimization. In Field-Programmable Custom Computing Machines, 2000 IEEE Symposium on, pages 307-308. IEEE, 2000.

[CCL03a] George A Constantinides, Peter YK Cheung, and Wayne Luk. Synthesis of saturation arithmetic architectures. ACM Transactions on Design Automation of Electronic Systems (TODAES), 8(3):334-354, 2003.

[CCL03b] George A Constantinides, Peter YK Cheung, and Wayne Luk. Wordlength optimization for linear digital signal processing. Computer-Aided Design of Integrated Circuits and Systems, IEEE Transactions on, 22(10):1432-1442, 2003.

[CCLF10] Gabriel Caffarena, Carlos Carreras, Juan A López, and Ángel Fernández. Sqnr estimation of fixed-point dsp algorithms. EURASIP Journal on Advances in Signal Processing, 2010:21, 2010.

[CDMV88] Francky Catthoor, Hugo De Man, and Joos Vandewalle. Simulatedannealing-based optimization of coefficient and data word-lengths in digital filters. International journal of circuit theory and applications, 16(4):371390, 1988 .

[CFCNT04] G Caffarena, A Fernandez, C Carreras, and O Nieto-Taladriz. Fixed-point refinement of ofdm-based adaptive equalizers: An heuristic approach. In EUSIPCO. Conference, 2004.

[CGC05] Jonathan A Clarke, Altaf Abdul Gaffar, and George A Constantinides. Parameterized logic power consumption models for fpga-based arithmetic. In 
Field Programmable Logic and Applications, 2005. International Conference on, pages 626-629. IEEE, 2005.

[CGL ${ }^{+}$09] Jason Cong, Karthik Gururaj, Bin Liu, Chunyue Liu, Zhiru Zhang, Sheng Zhou, and Yi Zou. Evaluation of static analysis techniques for fixed-point precision optimization. In Field Programmable Custom Computing Machines, 2009. FCCM'09. 17th IEEE Symposium on, pages 231-234. IEEE, 2009 .

[CH02] Mark L Chang and Scott Hauck. Précis: A design-time precision analysis tool. In Field-Programmable Custom Computing Machines, 2002. Proceedings. 10th Annual IEEE Symposium on, pages 229-238. IEEE, 2002.

[CKLM02] Martin Coors, Holger Keding, Olaf Lüthje, and Heinrich Meyr. Design and dsp implementation of fixed-point systems. EURASIP journal on applied signal processing, 2002(1):908-925, 2002.

[CLL ${ }^{+}$09] Gabriel Caffarena, Juan A. López, Gerardo Leyva, Carlos Carreras, and Octavio Nieto-Taladriz. Architectural Synthesis of Fixed-Point DSP Datapaths Using FPGAs. International Journal of Reconfigurable Computing, 2009(ii):1-14, 2009.

[CLNT99] Carlos Carreras, Juan A López, and Octavio Nieto-Taladriz. Bit-width selection for data-path implementations. In System Synthesis, 1999. Proceedings. 12th International Symposium on, pages 114-119. IEEE, 1999.

[CM12] Gabriel Caffarena and Daniel Menard. Many-core parallelization of fixedpoint optimization of vlsi circuits through gpu devices. In Design and Architectures for Signal and Image Processing (DASIP), 2012 Conference on, pages 1-8. IEEE, 2012.

[CMJL05] Martin Clark, Mike Mulligan, Dave Jackson, and Darel Linebarger. Accelerating fixed-point design for mb-ofdm uwb systems. Comms Design (an EE times Community), 2005.

[Con03] George A Constantinides. Perturbation analysis for word-length optimization. In Field-Programmable Custom Computing Machines, 2003. FCCM 2003. 11th Annual IEEE Symposium on, pages 81-90. IEEE, 2003.

[CRS ${ }^{+}$99] Radim Cmar, Luc Rijnders, Patrick Schaumont, Serge Vernalde, and Ivo Bolsens. A methodology and design environment for dsp asic fixed point refinement. In Design, Automation and Test in Europe Conference and Exhibition 1999. Proceedings, pages 271-276. IEEE, 1999.

[CSL02] M-A Cantin, Y Savaria, and P Lavoie. A comparison of automatic word length optimization procedures. In Circuits and Systems, 2002. ISCAS 
2002. IEEE International Symposium on, volume 2, pages II-612. IEEE, 2002 .

[CSPL01] M-A Cantin, Yvon Savaria, D Prodanos, and P Lavoie. An automatic word length determination method. In Circuits and Systems, 2001. ISCAS 2001. The 2001 IEEE International Symposium on, volume 5, pages 53-56. IEEE, 2001.

[CT05] SC Chan and KM Tsui. Wordlength determination algorithms for hardware implementation of linear time invariant systems with prescribed output accuracy. In Circuits and Systems, 2005. ISCAS 2005. IEEE International Symposium on, pages 2607-2610. IEEE, 2005.

[CT07] SC Chan and KM Tsui. Wordlength optimization of linear time-invariant systems with multiple outputs using geometric programming. Circuits and Systems I: Regular Papers, IEEE Transactions on, 54(4):845-854, 2007.

[CVKF03] Joan Carletta, Robert Veillette, Frederick Krach, and Zhengwei Fang. Determining appropriate precisions for signals in fixed-point iir filters. In Proceedings of the 40th annual Design Automation Conference, pages 656-661. ACM, 2003.

[CW02] George A Constantinides and Gerhard J Woeginger. The complexity of multiple wordlength assignment. Applied mathematics letters, 15(2):137$140,2002$.

[DCALP98] Luc De Coster, Marleen Adé, Rudy Lauwereins, and J Peperstaraete. Code generation for compiled bit-true simulation for dsp application. In Proceedings of the 11th international symposium on System synthesis, pages 9-14. IEEE Computer Society, 1998.

$\left[\mathrm{DNP}^{+} 04\right]$ Bert J Debusschere, Habib N Najm, Philippe P Pébay, Omar M Knio, Roger G Ghanem, and Olivier P Le Maître. Numerical challenges in the use of polynomial chaos representations for stochastic processes. SIAM Journal on Scientific Computing, 26(2):698-719, 2004.

[DYSD14] Gaël Deest, Tomofumi Yuki, Olivier Sentieys, and Steven Derrien. Toward scalable source level accuracy analysis for floating-point to fixed-point conversion. In Proceedings of the International Conference on Computer-Aided Design. ACM, 2014.

[ELS $\left.{ }^{+} 13\right] \quad L u i s$ Esteban, Jesus Lopez, E Sedano, S Hernandez-Montero, Miriam Sanchez, et al. Quantization analysis of the infrared interferometer of the tj-ii stellarator for its optimized fpga-based implementation. Nuclear Science, IEEE Transactions on, 60(5):3592-3596, 2013. 
[ELSS12] Luis Esteban, Juan A. López, Enrique Sedano, and Miguel Sánchez. Quantization Analysis of the Infrared Interferometer of the TJ-II for its Optimized FPGA-based Implementation. In IEEE 18th Real Time Conference, RTC'12, Berkeley (California, USA), 2012.

[Est11] L. Esteban. High precision FPGA based phase meters for infrared interferometers fusion diagnostics. PhD thesis, Universidad Politécnica de Madrid, 2011.

[FCR02] Fang Fang, Tsuhan Chen, and Rob A Rutenbar. Floating-point bit-width optimization for low-power signal processing applications. In Acoustics, Speech, and Signal Processing (ICASSP), 2002 IEEE International Conference on, volume 3, pages III-3208. IEEE, 2002.

[Fio08] Paul D Fiore. Efficient approximate wordlength optimization. Computers, IEEE Transactions on, 57(11):1561-1570, 2008.

[FL99] Paul D Fiore and Li Lee. Closed-form and real-time wordlength adaptation. In Acoustics, Speech, and Signal Processing, 1999. Proceedings., 1999 IEEE International Conference on, volume 4, pages 1897-1900. IEEE, 1999.

[FM82] CM Fiduccia and RM Mattheyses. A linear-time heuristic for improving network partitions. Design Automation, 1982. 19th Conference, pages 241$247,1982$.

[FRC03] Claire F Fang, Rob A Rutenbar, and Tsuhan Chen. Fast, accurate static analysis for fixed-point finite-precision effects in dsp designs. In Proceedings of the 2003 IEEE/ACM international conference on Computer-aided design, page 275. IEEE Computer Society, 2003.

[FRPC03] Claire Fang Fang, Rob A Rutenbar, Markus Püschel, and Tsuhan Chen. Toward efficient static analysis of finite-precision effects in dsp applications via affine arithmetic modeling. In Proceedings of the 40th annual Design Automation Conference, pages 496-501. ACM, 2003.

[FSFI14] The Free Software Foundation Inc. What is free software? http://www . gnu.org/philosophy/free-sw.en.html, August 2014.

[GCC06] Altaf Abdul Gaffar, Jonathan A Clarke, and George A Constantinides. Powerbit-power aware arithmetic bit-width optimization. In Field Programmable Technology, 2006. FPT 2006. IEEE International Conference on, pages 289-292. IEEE, 2006.

[GML04] Altaf Abdul Gaffar, Oskar Mencer, and Wayne Luk. Unifying bit-width optimisation for fixed-point and floating-point designs. In Field-Programmable 
Custom Computing Machines, 2004. FCCM 2004. 12th Annual IEEE Symposium on, pages 79-88. IEEE, 2004.

[GS91] Roger G Ghanem and Pol D Spanos. Stochastic finite elements: a spectral approach, volume 387974563. Springer, 1991.

[Han75] Eldon R Hansen. A generalized interval arithmetic. In Interval mathematics, pages 7-18. Springer, 1975.

[HE06] Kyungtae Han and Brian L Evans. Optimum wordlength search using sensitivity information. EURASIP Journal on Applied Signal Processing, 2006:76-76, 2006.

[HEKC01] Kyungtae Han, Iksu Eo, Kyungsu Kim, and Hanjin Cho. Numerical wordlength optimization for cdma demodulator. In Circuits and Systems, 2001. ISCAS 2001. The 2001 IEEE International Symposium on, volume 4, pages 290-293. IEEE, 2001.

[HESJ04] Kyungtae Han, Brian L Evans, and Earl E Swartzlander Jr. Data wordlength reduction for low-power signal processing software. In Signal Processing Systems, 2004. SIPS 2004. IEEE Workshop on, pages 343-348. IEEE, 2004 .

[HMS07] Nicolas Hervé, Daniel Ménard, and Olivier Sentieys. About the importance of operation grouping procedures for multiple word-length architecture optimizations. In Reconfigurable Computing: Architectures, Tools and Applications, pages 191-200. Springer, 2007.

$\left[\mathrm{HNK}^{+} 04\right] \mathrm{T}$ Horiyama, M Nakanishi, S Kimura, et al. Minimization of fractional wordlength on fixed-point conversion for high-level synthesis. In Design Automation Conference, 2004. Proceedings of the ASP-DAC 2004. Asia and South Pacific, pages 80-85. IEEE, 2004.

[Inc14] Xilinx Inc. A generation ahead for smarter systems: 9 reasons why the Xilinx Zynq-7000 all programmable SoC platform is the smartest solution. Xilinx Backgrounder, 2014.

[Inc15] Xilinx Inc. AccelDSP Synthesis Tool. http://www.xilinx.com/tools/ acceldsp.htm, 2015.

[Jac70] Leland B Jackson. On the interaction of roundoff noise and dynamic range in digital filters*. Bell System Technical Journal, 49(2):159-184, 1970.

[JCC08] Ruzica Jevtic, Carlos Carreras, and Gabriel Caffarena. Fast and accurate power estimation of fpga dsp components based on high-level switching activity models. International Journal of Electronics, 95(7):653-668, 2008. 
[Jer84] C Jeruchim. Techniques for estimating the bit error rate in the simulation of digital communication systems. Selected Areas in Communications, IEEE Journal on, 2(1):153-170, 1984.

[JW98] M Jersak and M Willems. Fixed-point extended c compiler allows more efficient high-level programming of fixed-point dsps. In Proceedings of the International Conference on Signal Processing Applications and Technology (ICSPAT'98), 1998.

[Kar14] George Karypis. METIS - Serial Graph Partitioning and Fill-reducing Matrix Ordering. http://glaros.dtc.umn.edu/gkhome/metis/metis/ overview, 2014.

[KHKM11] Jin Kim, Inwook Hwang, Yong-Hyuk Kim, and Byung-Ro Moon. Genetic approaches for graph partitioning: a survey. In Proceedings of the 13th annual conference on Genetic and evolutionary computation, pages 473480. ACM, 2011.

[KK98] George Karypis and Vipin Kumar. A fast and high quality multilevel scheme for partitioning irregular graphs. SIAM Journal on scientific Computing, 20(1):359-392, 1998.

[KS01] Ki-Il Kum and Wonyong Sung. Combined word-length optimization and high-level synthesis of digital signal processing systems. ComputerAided Design of Integrated Circuits and Systems, IEEE Transactions on, 20(8):921-930, 2001.

[Lat02] C.A. Lattner. LLVM: An infrastructure for multi-stage optimization. Master's thesis, 2002.

[LCCNT04] Juan A López, Gabriel Caffarena, Carlos Carreras, and Octavio NietoTaladriz. Analysis of limit cycles by means of affine arithmetic computeraided tests. In EUSIPCO. Conference, 2004.

[LCCNT08] Juan A. López, Gabriel Caffarena, Carlos Carreras, and Octavio NietoTaladriz. Fast and accurate computation of the round-off noise of linear time-invariant systems. IET Circuits, Devices \& Systems, 2(4):393, August 2008.

[LCNT07] Juan A López, Carlos Carreras, and Octavio Nieto-Taladriz. Improved interval-based characterization of fixed-point lti systems with feedback loops. Computer-Aided Design of Integrated Circuits and Systems, IEEE Transactions on, 26(11):1923-1933, 2007. 
[LGC $\left.{ }^{+} 06\right]$ D-U Lee, A Abdul Gaffar, Ray CC Cheung, Oskar Mencer, Wayne Luk, and George A Constantinides. Accuracy-guaranteed bit-width optimization. Computer-Aided Design of Integrated Circuits and Systems, IEEE Transactions on, 25(10):1990-2000, 2006.

[LGML05] Dong-U Lee, Altaf Abdul Gaffar, Oskar Mencer, and Wayne Luk. Minibit: bit-width optimization via affine arithmetic. In Proceedings of the 42nd annual Design Automation Conference, pages 837-840. ACM, 2005.

[Li09] Song Li. Fast algorithms for sparse matrix inverse computations. $\mathrm{PhD}$ thesis, Citeseer, 2009.

[Ló04] Juan A. López. Evaluación de los Efectos de Cuantificación en las Estructuras de Filtros Digitales Mediante Técnicas de Simulación Basadas en Extensiones de Intervalos. PhD thesis, 2004.

[May14] Javier Rodríguez Mayor. Optimización automática de algoritmos para cuantificación de circuitos eletrónicos digitales mediante procesadores gráficos. B.S. Thesis, ETSI Telecomunicación UPM, 2014.

[MB03] Kyoko Makino and Martin Berz. Taylor models and other validated functional inclusion methods. International Journal of Pure and Applied Mathematics, 4(4):379-456, 2003.

[MCS06] Daniel Menard, Daniel Chillet, and Olivier Sentieys. Floating-to-fixed-point conversion for digital signal processors. EURASIP journal on applied signal processing, 2006:77-77, 2006.

[Moo66] Ramon E Moore. Interval analysis, volume 4. Prentice-Hall Englewood Cliffs, 1966.

[MRS08] Daniel Menard, Romuald Rocher, and Olivier Sentieys. Analytical fixedpoint accuracy evaluation in linear time-invariant systems. Circuits and Systems I: Regular Papers, IEEE Transactions on, 55(10):3197-3208, 2008.

[MS00] V John Mathews and Giovanni L Sicuranza. Polynomial signal processing, volume 27. Wiley-Interscience, 2000.

[MSBZ07] Arindam Mallik, Debjit Sinha, Prithviraj Banerjee, and Hai Zhou. Lowpower optimization by smart bit-width allocation in a systemc-based asic design environment. Computer-Aided Design of Integrated Circuits and Systems, IEEE Transactions on, 26(3):447-455, 2007.

[MSNS12] Daniel Ménard, Naaa Simon, Jean Claude Naud, and Olivier Sentieys. Id.fix infrastructure for the design of fixed-point systems. In University Booth of the IEEE/ACM on Design Automation Conference (DAC). IEEE/ACM, 2012. 
[NLC $\left.{ }^{+} 96\right]$ SC Ng, SH Leung, CY Chung, A Luk, and WH Lau. The genetic search approach. a new learning algorithm for adaptive iir filtering. Signal Processing Magazine, IEEE, 13(6):38-46, 1996.

[NMS11] HN Nguyen, D Menard, and O Sentieys. Novel algorithms for word-length optimization. In Proc. European Signal Processing Conference (EUSIPCO), Barcelona, Spain, pages 1944-1948, 2011.

[NTA $\left.{ }^{+} 13\right]$ David Novo, I Tzimi, Ubaid Ahmad, Paolo Ienne, and Francky Catthoor. Cracking the complexity of fixed-point refinement in complex wireless systems. In Signal Processing Systems (SiPS), 2013 IEEE Workshop on, pages 18-23. Ieee, 2013.

[NTM92] R Nambiar, CKK Tang, and P Mars. Genetic and learning automata algorithms for adaptive digital filters. In Acoustics, Speech, and Signal Processing, 1992. ICASSP-92., 1992 IEEE International Conference on, volume 4, pages 41-44. IEEE, 1992.

$\left[\mathrm{OCC}^{+} 07\right]$ William G Osborne, J Coutinho, Ray CC Cheung, Wayne Luk, and Oskar Mencer. Instrumented multi-stage word-length optimization. In FieldProgrammable Technology, 200\%. ICFPT 200\%. International Conference on, pages 89-96. IEEE, 2007.

[ÖNG08] Emre Özer, Andy P Nisbet, and David Gregg. A stochastic bitwidth estimation technique for compact and low-power custom processors. ACM Transactions on Embedded Computing Systems (TECS), 7(3):34, 2008.

[Pah99] K Pahri. VLSI digital signal processing systems. John WileyÉ Sons, 1999.

[Par12] Karthick N. Parashar. System-level Approaches for Fixed-point Refinement of Signal Processing Algorithms. PhD thesis, 2012.

[PH06] Yu Pu and Yajun Ha. An automated, efficient and static bit-width optimization methodology towards maximum bit-width-to-error tradeoff with affine arithmetic model. In Proceedings of the 2006 Asia and South Pacific Design Automation Conference, pages 886-891. IEEE Press, 2006.

[PM98] Thomas M Panicker and VJ Mathew. Parallel-cascade realizations and approximations of truncated volterra systems. Signal Processing, IEEE Transactions on, 46(10):2829-2832, 1998.

[PRMS10a] Karthick Parashar, Romuald Rocher, Daniel Menard, and Olivier Sentieys. Analytical approach for analyzing quantization noise effects on decision operators. In Acoustics Speech and Signal Processing (ICASSP), 2010 IEEE International Conference on, pages 1554-1557. IEEE, 2010. 
[PRMS10b] Karthick Parashar, Romuald Rocher, Daniel Menard, and Olivier Sentieys. A hierarchical methodology for word-length optimization of signal processing systems. In VLSI Design, 2010. VLSID'10. 23rd International Conference on, pages 318-323. IEEE, 2010.

[PRZ10] Yu Pang, Kartazyna Radecka, and Zeljko Zilic. Optimization of imprecise circuits represented by taylor series and real-valued polynomials. ComputerAided Design of Integrated Circuits and Systems, IEEE Transactions on, 29(8):1177-1190, 2010.

[RB05] Sanghamitra Roy and Prith Banerjee. An algorithm for trading off quantization error with hardware resources for matlab-based fpga design. Computers, IEEE Transactions on, 54(7):886-896, 2005.

[RMHS06] Romuald Rocher, Daniel Menard, Nicolas Herve, and Olivier Sentieys. Fixed-point configurable hardware components. EURASIP Journal on Embedded Systems, 2006(1):20-20, 2006.

[SA05] Nasri Sulaiman and Tughrul Arslan. A multi-objective genetic algorithm for on-chip real-time optimisation of word length and power consumption in a pipelined fft processor targeting a mc-cdma receiver. In Evolvable Hardware, 2005. Proceedings. 2005 NASA/DoD Conference on, pages 154-159. IEEE, 2005 .

[SB04a] Changchun Shi and Robert W Brodersen. Automated fixed-point datatype optimization tool for signal processing and communication systems. In Design Automation Conference, 2004. Proceedings. 41st, pages 478-483. IEEE, 2004.

[SB04b] Changchun Shi and Robert W Brodersen. Floating-point to fixed-point conversion with decision errors due to quantization. In Acoustics, Speech, and Signal Processing, 2004. Proceedings.(ICASSP'04). IEEE International Conference on, volume 5, pages V-41. IEEE, 2004.

[SB04c] Changchun Shi and Robert W Brodersen. A perturbation theory on statistical quantization effects in fixed-point dsp with non-stationary inputs. In Circuits and Systems, 2004. ISCAS'04. Proceedings of the 2004 International Symposium on, volume 3, pages III-373. IEEE, 2004.

[SDF97] Jorge Stol and Luiz Henrique De Figueiredo. Self-validated numerical methods and applications. In Monograph for 21st Brazilian Mathematics Colloquium, IMPA, Rio de Janeiro. Citeseer, 1997.

[SEKH08] M Sánchez, L Esteban, P Kornejew, and M Hirsch. Admissible crosstalk limits in a two colour interferometers for plasma density diagnostics. a reduction algorithm. In PLASMA 200\%: International Conference on Research 
and Applications of Plasmas; 4th German-Polish Conference on Plasma Diagnostics for Fusion and Applications; 6th French-Polish Seminar on Thermal Plasma in Space and Laboratory, volume 993, pages 187-190. AIP Publishing, 2008.

[SJD $\left.{ }^{+} 12\right]$ Daniel Stutzbach, Jim J. Jewett, Fred Drake, Andrew Kuchling, and Thomas Wouters. Time complexity - The Python wiki. http://wiki.python. org/moin/TimeComplexity, August 2012.

[SK94] Wonyong Sung and Ki-Il Kum. Word-length determination and scaling software for a signal flow block diagram. In Acoustics, Speech, and Signal Processing, 1994. ICASSP-94., 1994 IEEE International Conference on, volume 2, pages II-457. IEEE, 1994.

[SK95] Wonyong Sung and Ki-Il Kum. Simulation-based word-length optimization method for fixed-point digital signal processing systems. Signal Processing, IEEE Transactions on, 43(12):3087-3090, 1995.

$\left[\mathrm{SLC}^{+} 12 \mathrm{a}\right]$ Enrique Sedano, Juan López, Carlos Carreras, et al. Acceleration of montecarlo simulation-based quantization of dsp systems. In Systems, Signals and Image Processing (IWSSIP), 201219 th International Conference on, pages 189-192. IEEE, 2012.

[SLC $\left.{ }^{+} 12 \mathrm{~b}\right]$ Enrique Sedano, Juan López, Carlos Carreras, et al. A fast interpolative wordlength optimization method for dsp systems. In Programmable Logic (SPL), 2012 VIII Southern Conference on, pages 1-6. IEEE, 2012.

[SLMW03] Huahao Shou, Hongwei Lin, Ralph Martin, and Guojin Wang. Modified Affine Arithmetic Is More Accurate than Centered Interval Arithmetic or Affine Arithmetic. In Michael J. Wilson and Ralph R. Martin, editors, Mathematics of Surfaces, volume 2768 of Lecture Notes in Computer Science, pages 355-365. Springer Berlin Heidelberg, Berlin, Heidelberg, 2003.

[SLMW04] Huahao Shou, Hongwei Lin, Ralph Martin, and Guojin Wang. Modified Affine Arithmetic in Tensor Form. In Computing and Information, Int.Symp. on, pages 642-646, 2004.

[SML14] Enrique Sedano, Daniel Menard, and Juan A López. Automated data flow graph partitioning for a hierarchical approach to wordlength optimization. In Reconfigurable Computing: Architectures, Tools, and Applications, pages 133-143. Springer, 2014.

[SPR10] O Sarbishei, Y Pang, and K Radecka. Analysis of range and precision for fixed-point linear arithmetic circuits with feedbacks. In High Level Design Validation and Test Workshop (HLDVT), 2010 IEEE International, pages 25-32. IEEE, 2010. 
[SR10] O Sarbishei and K Radecka. Analysis of precision for scaling the intermediate variables in fixed-point arithmetic circuits. In Proceedings of the International Conference on Computer-Aided Design, pages 739-745. IEEE Press, 2010.

[SR12] O Sarbishei and K Radecka. Fixed-point accuracy analysis of datapaths with mixed cordic and polynomial computations. In Design Automation Conference (ASP-DAC), 2012 17th Asia and South Pacific, pages 789-794. IEEE, 2012.

[SR13] Omid Sarbishei and Katarzyna Radecka. On the fixed-point accuracy analysis and optimization of polynomial specifications. Computer-Aided Design of Integrated Circuits and Systems, IEEE Transactions on, 32(6):831-844, 2013.

[TNR00] Jonathan Ying Fai Tong, David Nagle, and Rob A Rutenbar. Reducing power by optimizing the necessary precision/range of floating-point arithmetic. Very Large Scale Integration (VLSI) Systems, IEEE Transactions on, 8(3):273-286, 2000.

[WBGM97] Markus Willems, Volker Bursgens, Thorsten Grotker, and Heinrick Meyr. Fridge: an interactive code generation environment for hw/sw codesign. In Acoustics, Speech, and Signal Processing, 1997. ICASSP-97., 1997 IEEE International Conference on, volume 1, pages 287-290. IEEE, 1997.

[WDJ $\left.{ }^{+} 06\right]$ Jan-Willem Weijers, Veerle Derudder, Sven Janssens, Frederik Petré, and André Bourdoux. From mimo-ofdm algorithms to a real-time wireless prototype: a systematic matlab-to-hardware design flow. EURASIP journal on applied signal processing, 2006:138-138, 2006.

[WK05] Xiaoliang Wan and George Em Karniadakis. An adaptive multi-element generalized polynomial chaos method for stochastic differential equations. Journal of Computational Physics, 209(2):617-642, November 2005.

[WK06] Xiaoliang Wan and George Em Karniadakis. Multi-element generalized polynomial chaos for arbitrary probability measures. SIAM Journal on Scientific Computing, 28(3):901-928, 2006.

[WP98] Suhrid A Wadekar and Alice C Parker. Accuracy sensitive word-length selection for algorithm optimization. In Computer Design: VLSI in Computers and Processors, 1998. ICCD'98. Proceedings. International Conference on, pages 54-61. IEEE, 1998.

[Wu12] Bin Wu. Dynamic range estimation for systems with control-flow structures. In Quality Electronic Design (ISQED), 2012 13th International Symposium on, pages 370-377. IEEE, 2012. 
[WZN04a] Bin Wu, Jianwen Zhu, and Farid N Najm. An analytical approach for dynamic range estimation. In Proceedings of the 41st annual Design Automation Conference, pages 472-477. ACM, 2004.

[WZN04b] Bin Wu, Jianwen Zhu, and Farid N Najm. Dynamic range estimation for nonlinear systems. In Proceedings of the 2004 IEEE/ACM International conference on Computer-aided design, pages 660-667. IEEE Computer Society, 2004.

[WZN06] Bin Wu, Jianwen Zhu, and Farid N Najm. Dynamic-range estimation. Computer-Aided Design of Integrated Circuits and Systems, IEEE Transactions on, 25(9):1618-1636, 2006.

[XK02] Dongbin Xiu and George Em Karniadakis. The Wiener-Askey Polynomial Chaos for Stochastic Differential Equations. SIAM Journal on Scientific Computing, 24(2):619-644, January 2002.

[XK03] Dongbin Xiu and George Em Karniadakis. Modeling uncertainty in flow simulations via generalized polynomial chaos. Journal of computational physics, 187(1):137-167, 2003.

[XL02] Dongbin Xiu and Didier Lucor. Stochastic modeling of flow-structure interactions using generalized polynomial chaos. Journal of Fluids Engineering, 124(1):51-59, 2002.

[ZB97] Charles N Zeeb and Patrick J Burns. A comparison of failure probability estimates by monte carlo sampling and latin hypercube sampling. Sandia National Laboratories, 1997.

[ZR09] Zeljko Zilic and Katarzyna Radecka. Enabling practical uses of arithmetic transform: a comprehensive analysis. In Proceedings of 9th international workshop on Reed-Muller transform and its applications, $R M$, volume 9 , 2009 . 
I shouldn't believe anything I say, if I were you-and that includes what I just told you.

Jasper Fforde,

The Eyre Affair 